\title{
Shorter Contributions
}

to General Geology

1958

GEOLOGICAL SURVEY PROFESSIONAL PAPER 334

This Professional Paper was published

as separate chapters $A-H$

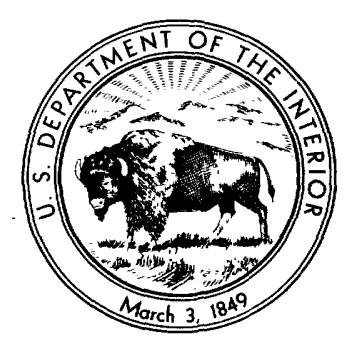

UNITED STATES GOVERNMENT PRINTING OFFICE, WASHINGTON : 1962 
UNITED STATES DEPARTMENT OF THE INTERIOR

STEWART L. UDALL, Secretary

GEOLOGIGAL SURVEY

Thomas B. Nolan, Director

For sale by the Superintendent of Documents, U.S. Government Printing Office Washington 25, D.C. 


\section{CONTENTS}

[The letters in parentheses are those used to designate the separate chapters]

(A) Tables for the calculation of lead isotope ages, by L. R. Stieff, T. W. Stern, Seiki Oshiro, and F. E. Senftle

(B) Fossils of the Littleton formation (Lower Devonian) of New Hampshire, by A. J. Boucot and Robert Arndt..........

(C) Trilobites of the Upper Cambrian Dunderberg shale, Eureka district, Nevada, by Allison R. Palmer.............

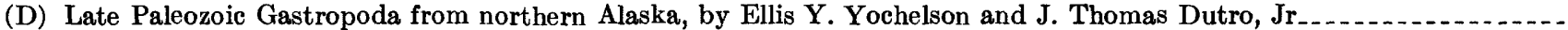

(E) Upper Cretaceous Pelecypods of the genus Inoceramus from northern Alaska, by David L. Jones and George Gryc...--

(F) Ammonites of Early Cretaceous age (Valanginian and Hauterivian) from the Pacific Coast States, by Ralph W. Imlay -

(G) Dispersion characteristics of montmorillonite,kaolinite, and illite clays in waters of varying quality, and their control with phosphate dispersants, by B. N. Rolfe, R. F. Miller, and I. S. MeQueen

(H) Geology of southeastern Ventura basin, Los Angeles County, California, by E. L. Winterer and D. L. Durham.-.-.-- 


\section{Tables for the Calculation}

of Lead Isotope Ages

By L. R. STIEFF, T. W. STERN, SEIKI OSHIRO, and F. E. SENFTLE

SHORTER CONTRIBUTIONS TO GENERAL GEOLOG"r

GEOLOGICAL SURVEY PROFESSIONAL PAPER 334-A

Tables for the calculation of geologic age using the atomic ratios of $\mathrm{Pb}^{206} / \mathrm{U}^{238}, \mathrm{~Pb}^{207} / \mathrm{U}^{235}, \mathrm{~Pb}^{207} / \mathrm{Pb}^{206}$, and $\mathrm{Pb}^{208} / \mathrm{Th}^{232}$. This report concerns work done on behalf of the U.S. Atomic Energy Commission and is published with the permission of the Commission

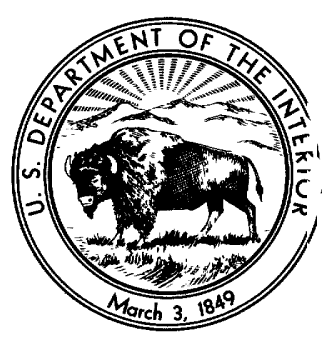

UNITED STATES GOVERNMENT PRINTING OFFICE, WASHINGTON : 1959 
UNITED STATES DEPARTMENT OF THE INTERIOR

FRED A. SEATON, Secretary

GEOLOGICAL SURVEY

Thomas B. Nolan, Director

The U. S. Geological Survey Library has cataloged this publication as follows :

Stieff, Lorin Rollins, 1920-

Tables for the calculation of lead isotope ages, by L. R. Stieff ${ }_{\text {[ }}$ and others] Washington, U. S. Govt. Print. Off., 1959.

iv, 40 p. diagrs., tables. $30 \mathrm{~cm}$. (U. S. Geological Survey. Professional paper 334-A. Shorter contributions to general geology)

"Tables for the calculation of geologic age using the atomic ratios of $\mathrm{Pb} 206 / \mathrm{U} 238$, Pb207/U235, Pb207/Pb206, and Pb208/Th232."

Bibliography : p. 7.

1. Lead - Isotopes. 2. Isotopes - Tables, etc. 3. Geological time I. Title. (Series: U. S. Geological Survey. Professional paper 334-A. Series: U. S. Geological Survey. Shorter contributions to general geology)

550.1

For sale by the Superintendent of Documents, U. S. Government Printing Office Washington 25, D. C. - Price 35 cents (paper copy) 


\section{CONTENTS}

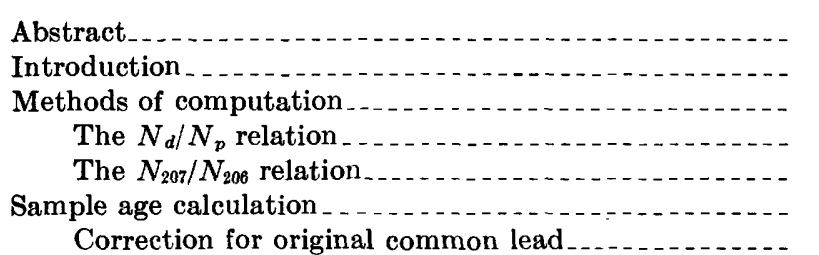

Page

1

Sample age calculation-Continued age method

$\mathrm{Pb}^{207} / \mathrm{U}^{235}$ age method $\ldots$

$\mathrm{Pb}^{207} / \mathrm{Pb}^{206}$ age method....... 7

$\mathrm{Pb}^{208} / \mathrm{Th}^{232}$ age method. 7

Literature cited..... 7

Tables for the calculation of lead isotope ages 9

\section{ILLUSTRATIONS}

Figure 1. Atomic ratio $N_{d} / N_{p}$ plotted against the age, $t$, showing the plus and minus tolerance curves

2. Atomic ratio $N_{207} / N_{206}$ plotted against the age, $t$, showing the plus and minus tolerance curves

\section{TABLES}

TABLE 1. Physical constants used in calculation of tables.

2. Errors in $t$ produced by uncertainties in the physical constants used in calculation

3. Range and interval of $t$

4. Decay constants $\lambda_{i_{j}}\left(y^{-1}\right)$ 


\section{LETTER SYMBOLS}

$\lambda$ decay constant

$t \quad$ age, in millions of years

$T \quad$ half life

$N$ number of atoms

$N_{d} \quad$ number of atoms of daughter products

$N_{p} \quad$ number of atoms of parent

$k$ present-day atomic ratio of $\mathrm{U}^{238}$ to $\mathrm{U}^{235}$

$y \quad$ year

$R \quad$ atomic ratio 


\title{
TABLES FOR THE GALGULATION OF LEAD ISOTOPE AGES
}

\author{
By L. R. Stieff, T. W. Stern, Seiki Oshiro, and F. E. Senftle
}

ABSTRACT

Tables are presented for calculating geologic age by using the atomic ratios of $\mathrm{Pb}^{208} / \mathrm{U}^{238}, \mathrm{~Pb}^{207} / \mathrm{U}^{235}, \mathrm{~Pb}^{207} / \mathrm{Pb}^{206}$, and $\mathrm{Pb}^{208} / \mathrm{Th}^{232}$.

Tables of values of $N_{d} / N_{p}$ and $t$ are given for the age equation

$$
\frac{N_{d}}{N_{p}}=\exp \lambda t-1
$$

where $\lambda$ is the decay constant,

$t$ is age, in millions of years,

$N_{d}$ is the number of atoms of daughter products,

and $N_{D}$ is the number of atoms of parent.

Values for $N_{207} / N_{208}$ and $t$ are also given in tabular form for the age equation

$$
\frac{N_{207}}{N_{206}}=\frac{\exp \lambda_{235} t-1}{k\left(\exp \lambda_{238} t-1\right)}
$$

where $N_{207}$ and $N_{206}$ are the number of atoms of radiogenic $\mathrm{Pb}^{207}$ and $\mathrm{Pb}^{206}$, respectively, and where $k$, the present-day atomic ratio of $U^{238}$ to $U^{235}$, is taken as 137.7. The half lives $(T)$ of $\mathrm{U}^{238}$, $\mathrm{U}^{235}$, and $\mathrm{Th}^{232}$ used in the calculations are: $T_{238}=4.51 \times 10^{9}$ years, $T_{235}=7.13 \times 10^{8}$ years, $T_{232}=1.42 \times 10^{10}$ years.

The tables cover selected values of $t$ from 1 to 6,000 million years $\left(6 \times 10^{9}\right.$ years) at intervals of $t$ ranging from 1 to 15 million years. Only the calculated errors in $t$ resulting from experimental uncertainties in the determinations of the decay constants and relative abundance of $\mathrm{U}^{238}$ and $\mathrm{U}^{235}$ have been included. An example is given for a hypothetical geologic age calculation by use of these tables.

\section{INTRODUCTION}

Although lead isotope age calculations are in general not difficult to make, both graphs and nomographs are available in the literature to simplify these computations. Holmes (1931, p. 208) published in "The Age of the Earth" a graphical solution of the age equation involving total lead, uranium, and thorium. Eight years later Wickman $(1939$, p. 6) published several nomographs that gave ages in millions of years equivalent to the weight ratios of $\mathrm{Pb}^{206} / \mathrm{U}^{238}, \mathrm{~Pb}^{207} / \mathrm{Pb}^{206}$, and $\mathrm{Pb}^{208} / \mathrm{Th}^{232}$. More recently, Kulp and others (1954, $\mathrm{p}$. 345) have prepared nomographs of the three age ratios mentioned above as well as the ratios of $\mathrm{Pb}^{207} / \mathrm{U}^{235}$ in terms of numbers of atoms rather than in weight percent.
During the course of geochronological studies $m \varepsilon$,de by the U. S. Geological Survey it was found desirable to have age equivalents for the various lead isotope ratios at smaller intervals and over a greater range of time than could be obtained from the references mentioned above. Initially, these tables were calculated for the atomic ratios of $\mathrm{Pb}^{208} / \mathrm{U}^{238}, \mathrm{~Pb}^{207} / \mathrm{U}^{235}, \mathrm{~Pb}^{207} / \mathrm{Pb}^{206}$, and $\mathrm{Pb}^{208} / \mathrm{Th}^{232}$ at intervals of $t$ of 1 million years from 1 million years to 6,000 million years. The tables in this paper are an abridgment of the original tables. The calculations were carried to 6,000 million years, an age greater than the probable age of the earth, so that speculative calculations could be made. They are being published because it has been found that for certain types of analysis of isotopic age data these tables are more satisfactory than the published nornographs. For example, the tables offer greater accuracy and ease of manipulation than the nomographs in making repeated solutions of the age equations. Such repeated calculations are particularly useful in evaluating different geologic processes which have produced discordant ages.

The general form of the age equations used to compute the tables was developed by Kovarik (1931, p. 73), Keevil (1939, p. 195), and others. Solutions were obtained for equations of the form

The equation

$$
\frac{N_{d}}{N_{p}}=\exp \lambda t-1
$$

$$
\frac{N_{207}}{N_{206}}=\frac{\exp \lambda_{235} t-1}{k\left(\exp \lambda_{238} t-1\right)}
$$

was solved by iteration. It is important to note that the ratios of $N_{d} / N_{p}$ and $N_{207} / N_{206}$ in the present tal' es are given as ratios of number of atoms of daughter $\left(N_{d}\right)$ to number of atoms of parent $\left(N_{p}\right)$, and number of atoms of $\mathrm{Pb}^{207}\left(N_{207}\right)$ to $\mathrm{Pb}^{206}\left(N_{206}\right)$ and not in terms of weight percent.

The authors wish to express their appreciation to F. W. Reilly and D. B. Rock of the Computer Branch for their aid in the operation of the computer, and also 
to Raynor L. Duncomb, Julena S. Duncomb and G. M. Clemence, of the U. S. Naval Observatory, for their cooperation and help in preparing the tables for printing. This work is part of a program being conducted by the U. S. Geological Survey on behalf of the Division of Research of the U. S. Atomic Energy Commission.

The half lives, decay constants, and ratio of $\mathrm{U}^{238} / \mathrm{U}^{235}$ used in the calculations are shown in table 1, along with those used by Wickman and by.Kulp and others. The values used in this paper were chosen for the following reasons. Fleming and others $(1952$, p. 642$)$ discuss in some detail the earlier work on the determination of the half life of $\mathrm{U}^{235}$. The weighted average of all previous measurements is numerically th 9 same as their value with a slightly larger probable error. The halflife data of Fleming and others was therefore chosen for these calculations. These authors have also discussed the past determinations of the lalf life of $\mathrm{U}^{238}$. From their "best values" a half life of $(4.51 \pm 0.007) \times$ $10^{9}$ years has been calculated. More recently Kovarik and Adams (1955, p. 46) have redetermined three of their original specimens. Their newest value, $(4.507 \pm$ $0.009) \times 10^{9}$ years, lies within the limits of error of the "best value" calculation of Fleming and others. In view of the small differences in half lives, as well as the differences in probable error, a value of $(4.51 \pm 0.01) \times$ $10^{9}$ years was therefore chosen.

TABLE 1.-Physical constants used in calculation of tables

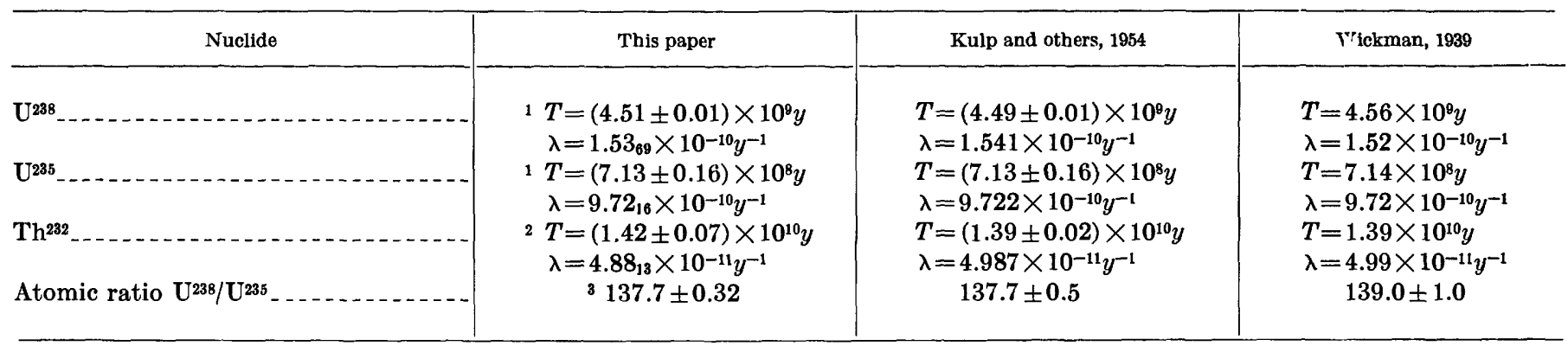

1 Fleming and others, 1952 , p. 642.

2 Senftle, Farles, and Lazar, 1956, p. 1629.

3 Senftle, Stieff, Cuttitta, and Kuroda, 1957, p. 189

Kovarik and Adams (1938, p. 413) have also determined the half life of $\mathrm{Th}^{232}$ as $(1.39 \pm .03) \times 10^{10}$ years. In their very thorough and excellent paper they determined in addition the branching ratio of $\mathrm{Bi}^{212}$. Recently this branching ratio and the half life of thorium have been redetermined by Senftle, Farley, and Lazar (1956). Using a pulse-counting technique they obtained a branching ratio 7.4 percent higher than that obtained by Kovarik and Adams. Although the half life as determined by Kovarik and Adams does not directly depend on the branching ratio of $\mathrm{Bi}^{212}$, the half life was determined from the basic alpha-count data. In spite of the careful alpha-counting techniques used by Kovarik and Adams, the differences in the branching ratio of $\mathrm{Bi}^{212}$ raise some questions on the earlier $\mathrm{Th}^{232}$ half-life determinations. Also, their calculations depended on the existence of radioactive equilibrium between $\mathrm{Th}^{232}$ and $\mathrm{Th}^{228}$ based on the age of the thorite and the assumption that the daughter products were undisturbed by processes of alteration and weathering. Senftle, Farley, and Lazar, however, have shown that even for a specimen of fresh thorite chosen because it showed no signs of alteration, the $\mathrm{Ra}^{224}$ was 9.5 percent less than the equilibrium amounts; this implies a loss of its parents $\mathrm{Th}^{228}$ and $\mathrm{Ra}^{228}$. The tendency of radiogenic daughter products to migrate has been pointed out in detail by Rosholt (1958). Hence, the value of the half life $(1.42 \pm 0.07) \times 10^{10}$ years as determined by Senftle and others was used, even thcugh the quoted percent of error is somewhat larger than that of Kovarik and Adams.

The determination of the atomic ratio $\mathrm{N}_{238} / \mathrm{N}_{235}$ has been discussed by Fleming and others (1952, p. 642). They observe that a mean value of 137.7 "falls within the limits of error of all values reported." Kulp and others $(1954$, p. 345$)$ used a value of $137.7 \pm 0.5$. More recently Senftle, Stieff, Cuttitta, and Kuroda (1957, p. 190) have shown an average value of $137.7 \pm$ 0.32 for 13 uranium specimens. This value with its somewhat smaller probable error has been used for these calculations.

In general, the ratios in the tables s,re given to four significant places, a value somewhat better than can be justified by present physical measurements and analytical methods. The remaining two numbers in smaller type have been included bocause of their usefulness in certain theoretical calculations. Corresponding to each ratio a value of $t$ plus or minus the error may be read from the tables or may be directly interpolated as a first approximation if the exact value of the ratio desired is not found. Tre limits of error for $t$ shown in the tables have been calculated by using only the limits of experimental error reported for 
the determinations of the decay constants and the abundance ratios, and the error term has been rounded to the nearest 0.1 million years. The error calculations are treated more fully in the section "Methods of computation."

TABLE 2.-Errors in $\mathrm{t}$ produced by uncertainties in the physical constants used in calculation

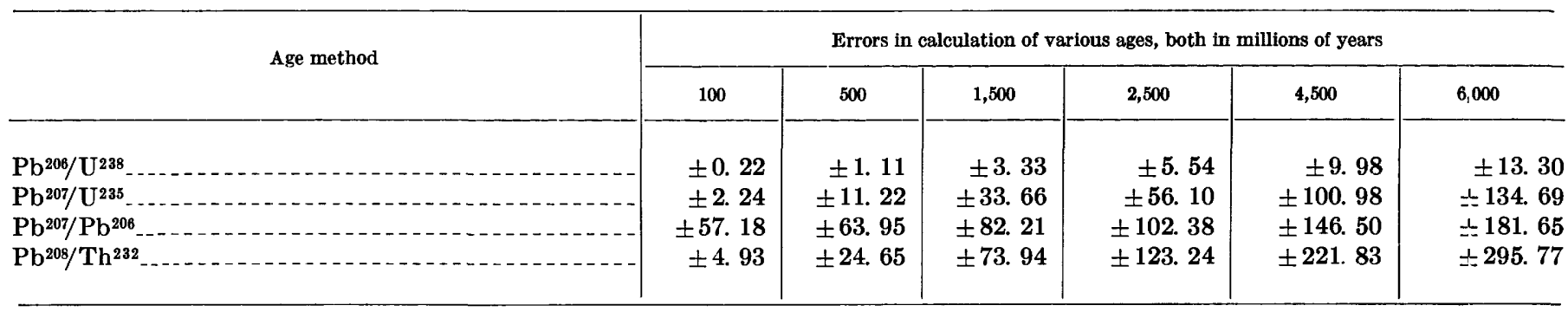

Table 2 lists for several different $t$ 's the limits of error in the age calculations resulting from the uncertainties in the physical constants used. This table shows that the limits of error in calculated age for the $\mathrm{Pb}^{206} / \mathrm{U}^{238}$ method are less than the limits of error for the other three methods. The selection of intervals of $t$ (table 3 ) was determined in part by the limits of error for the

TABLE 3.-Range and interval of $\mathrm{t}$

\begin{tabular}{|l|c|}
\hline \multicolumn{1}{|c|}{ Range (years) } & Interval (years) \\
\hline & \\
$1 \times 10^{6}$ to $500 \times 10^{6}$ & $1 \times 10^{6}$ \\
$500 \times 10^{6}$ to $1,500 \times 10^{6}$ & $2 \times 10^{6}$ \\
$1,500 \times 10^{6}$ to $2,500 \times 10^{6}$ & $5 \times 10^{6}$ \\
$2,500 \times 10^{6}$ to $4,500 \times 10^{6}$ & $10 \times 10^{6}$ \\
$4,500 \times 10^{6}$ to $6,000 \times 10^{6}$ & $15 \times 10^{6}$ \\
& \\
\hline
\end{tabular}

$\mathrm{Pb}^{208} / \mathrm{U}^{238}$ method. In spite of the larger errors inherent in the other methods, the same interval of $t$ has been used for all four tables in order that equivalent ratios might be obtained for the same value of $t$. Admittedly, the intervals chosen for the abridged tables are smaller that the uncertainties in the calculated ages introduced by the most precise analytical techniques currently available. However, improvements in the quantitative determination of lead, uranium, thorium, and isotopic abundance may ultimately permit the measurerent of small differences in age ( 1 to 2 million years) of radioactive minerals from rocks of Cambrian age or younger.

\section{METHODS OF COMPUTATION THE $N_{d} / N_{p}$ RELATION}

For the purpose of programing this work for the digital computer, the general form of the age ecuation was used,

$$
\frac{N_{d}}{N_{p}}=R=\exp \lambda_{i j} t-1
$$

where $i=1,2,3$

and $j=1,2,3$.

The values of the decay constants with their limits, $\lambda_{i_{j}}$, used for these calculations are shown in tab $b^{1}, 4$.

TABLE 4.-Decay constants $\lambda_{i_{i}}\left(\mathrm{y}^{-1}\right)$

\begin{tabular}{|c|c|c|}
\hline$\lambda$ & $\lambda$ plus error & $\lambda$ minus error \\
\hline & & \\
$\mathrm{U}^{238} \lambda_{1_{1}}=1.53_{69} \times 10^{-10}$ & $\lambda_{1_{2}}=1.53_{35} \times 10^{-10}$ & $\lambda_{1_{3}}=1.54_{03} \times 10^{-10}$ \\
$\mathrm{U}^{235} \lambda_{2_{1}}=9.72_{16} \times 10^{-10}$ & $\lambda_{2_{2}}=9.50_{82} \times 10^{-10}$ & $\lambda_{2_{3}}=9.94_{47} \times 10^{-10}$ \\
$\mathrm{Th}^{232} \lambda_{3_{1}}=4.88_{13} \times 10^{-11}$ & $\lambda_{3_{2}}=4.65_{20} \times 10^{-11}$ & $\lambda_{3_{3}}=5.13_{44} \times 10^{-11}$ \\
& & \\
\hline
\end{tabular}

In computing $\exp \lambda_{i j} t-1$ the exponential series was expanded

$$
\exp x-1=\sum_{n=1}^{\infty} \frac{x^{n}}{n !}=-\bar{x}+\frac{x^{2}}{2 !}+\frac{x^{3}}{3 !}
$$

and the recursion $\frac{x^{n-1}}{(n-1) !} \frac{x}{n}=\frac{x^{n}}{n !}$ was used to evaluate the series. No round off was used in the evaluation and the maximum error in $\exp \lambda_{i}, t$ can be shown to be less than $5 \times 10^{-5}$.

The problem of calculating the errors in the ages due to the uncertainties in the decay constants was simplified in the following graphical treatment shown in figure 1. By virtue of the geometry in figure 1,

$$
t_{1} N=t_{3} M=t_{2} \mathrm{O}
$$

where $t_{2}$ is the age for a plus error in $\lambda$ and $t_{3}$ is the age for a minus error in $\lambda$.

Therefore,

$$
\exp \lambda_{i_{1}} t_{1}-1=\exp \lambda_{i_{2}} t_{2}-1
$$

and

$$
\exp \lambda_{i_{1}} t_{1}-1=\exp \lambda_{i_{3}} t_{3}-1 \text {. }
$$




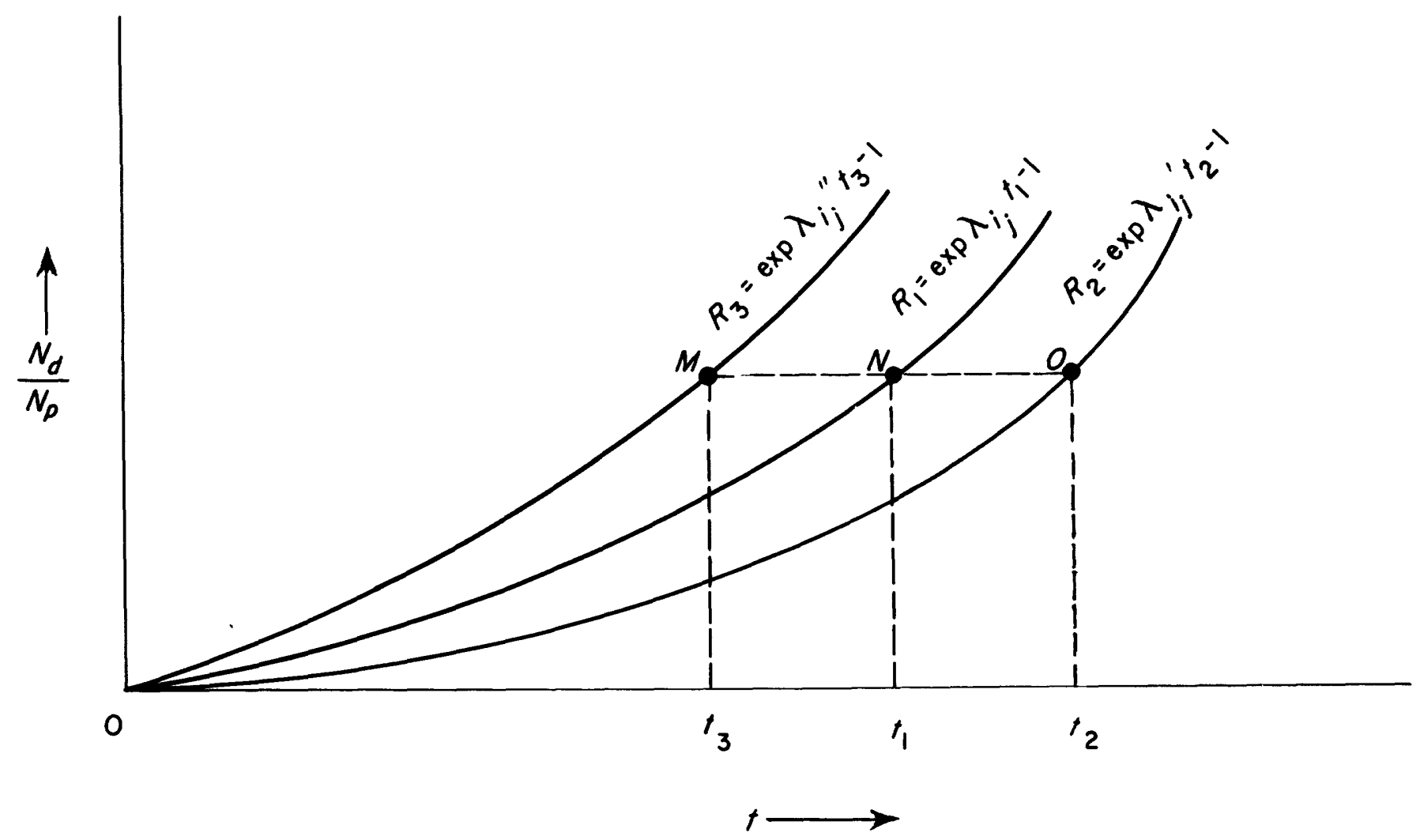

FIGURE 1.-Atomic ratio $N_{d} / N_{D}$ plotted against the age, $t$, showing the plus and minus tolerance curves.

Simplifying and taking logarithms,

$$
\lambda_{i_{1}} t_{1}=\lambda_{i_{2}} t_{2}
$$

and

$$
\lambda_{i_{1}} t_{1}=\lambda_{i_{3}} t_{3} \text {. }
$$

Also it is evident from figure 1 that $M N=t_{1}-t_{3}$ and $N O=t_{2}-t_{1}$. Thus, by the substitution the error in the age, $M N$ and $N O$, can be simply evaluated in a convenient form,

$$
M N=t_{1}\left(1-\frac{\lambda_{i_{1}}}{\lambda_{i_{3}}}\right)
$$

and

$$
N O=t_{1}\left(\frac{\lambda_{i_{1}}}{\lambda_{i_{2}}}-1\right)
$$

If new, more nearly accurate, values of the decay constants become available, it is a simple matter to obtain the corrected age from the tables. For example, suppose the half life of the $\mathrm{Th}^{232}$ is changed from $1.42 \pm$ $0.07 \times 10^{10}$ years to $1.45 \pm 0.02 \times 10^{10}$ years. Calculated in this way using $T$ instead of $\lambda$, the new value of the age, $t_{1}^{\prime}$ say, will be

$$
t_{1}{ }^{\prime}=\frac{1.45 t_{1}}{1.42}
$$

Similarly, $t_{2}^{\prime}$ and $t_{3}^{\prime}$ will be

$$
t_{2}^{\prime}=\frac{1.47 t_{1}}{1.42}
$$

and

$$
t_{3}{ }^{\prime}=\frac{1.43 t_{1}}{1.42}
$$

Then, if one assumes an experimental ratio of $N_{208} / N_{232}$ of $\left(0.0500_{23}\right)$, the corresponding value of $t_{1}\left(N_{208} / N_{232}\right.$ table) is $1,000 \times 10^{6}$ years. The new half-life value would yield $t_{1}{ }^{\prime}, t_{2}{ }^{\prime}$, and $t_{3}{ }^{\prime}$ ages of $1,021 \times 10^{6}$ years, $1,035 \times 10^{6}$ years, and $1,007 \times 10^{6}$ years, respectively. Thus, the tables can still be used even if new values of the half lives are redetermined at a later date.

\section{THE $\boldsymbol{N}_{207} / \boldsymbol{N}_{200}$ RELATION}

The general equation,

$$
\frac{N_{207}}{N_{206}}=R=\frac{\exp \lambda_{2_{1}} t-1}{k_{1}\left(\exp \lambda_{1_{1}} t-1\right)},
$$

was used for computing the $\mathrm{Pb}^{207} / \mathrm{Pb}^{208}$ table. The value, $k_{1}$, used for the $\mathrm{U}^{238} / \mathrm{U}^{235}$ atomic ratio was 137.7 \pm 0.32 where $k_{2}$ and $k_{3}$ designate the abur lance ratios with the plus and minus tolerances, respectively. 
As in the $N_{d} / N_{p}$ relation previously described, the calculation of limits of error in the age due to uncertainties in the decay constants has been simplified for programing on the digital computer. By use of a similar graphical argument as shown in figure 2, it can be seen that

$$
R=\frac{\exp \lambda_{2_{1}} t_{1}-1}{k_{1}\left(\exp \lambda_{1_{1}} t_{1}-1\right)}=\frac{\exp \lambda_{22} t_{2}-1}{k_{2}\left(\exp \lambda_{1_{2}} t_{2}-1\right)}
$$

and

$$
R=\frac{\exp \lambda_{2_{1}} t_{1}-1}{k_{1}\left(\exp \lambda_{1_{1}} t_{1}-1\right)}=\frac{\exp \lambda_{2_{3}} t_{3}-1}{k_{3}\left(\exp \lambda_{1_{3}} t_{3}-1\right)}
$$

However, unlike the previous solution, the equations $(7 \mathrm{a}, \mathrm{b})$ have no direct solution for $t_{2}$ and $t_{3}$ and an iterative-approximation method had to be used. The left-hand sides of the equations can be evaluated. The right-hand sides are quotients of infinite series in $t_{2}$ and $t_{3}$. An initial guess was made for $t_{2}$ or $t_{3}$, as the case may be, and a test for equality was made. Successive approximations were made to the $t$ values until equality was obtained.

For the particular case where $t_{1}=t_{2}=t_{3}=0$, the ratio $R$ is indeterminate. However, by using L'Hospital's ule an approximate but quite accurate value of $R$ can be obtained. Hence, the limits of the ratios as $t_{1}, t_{2}$, and $t_{3} \rightarrow 0$, are $R_{1}=0.0459_{36}, \quad R_{2}=0.0449$, ard $R_{3}$ $=0.0470$. Thus, radiogenic lead being formed at the present time should and does have a $N_{207} / N_{206}$ ratio between 0.045 and 0.047 , a value very close to the experimentally observed value.

From the age tables it can be seen that below an age of $56 \times 10^{6}$ years, the $\mathrm{Pb}^{207} / \mathrm{Pb}^{206}$ method has errors that are larger than the calculated value of $t$. As has been mentioned, this error is due only to the uncertainties in the physical constants used in the calculations. It is shown in figure 2 for an age $t$, (which is less than $56 \times 10^{6}$ years) that the horizontal line $(a-b)$ between the ages representing the plus and minus tolerances does not intersect the upper curve on the positive side of the coordinates because of the flatness of the curves in this region. Also, this "flatness" and the round-off error cause oscillation of approximately 0.1 to 1.0 million years in the quoted error in $t$ for ages less than 400 million years, and small irregularities in the 5 th and 6th places of the $N_{207} / N_{206}$ ratio for the range from 0 to 50 million years.

\section{SAMPLE AGE CALCULATION}

A hypothetical uraninite gave the following chemical data: $\mathrm{U}=43.6_{46}$ percent, $\mathrm{Pb}=7.53_{2}$ percent, $\mathrm{Th}=5.20_{1}$

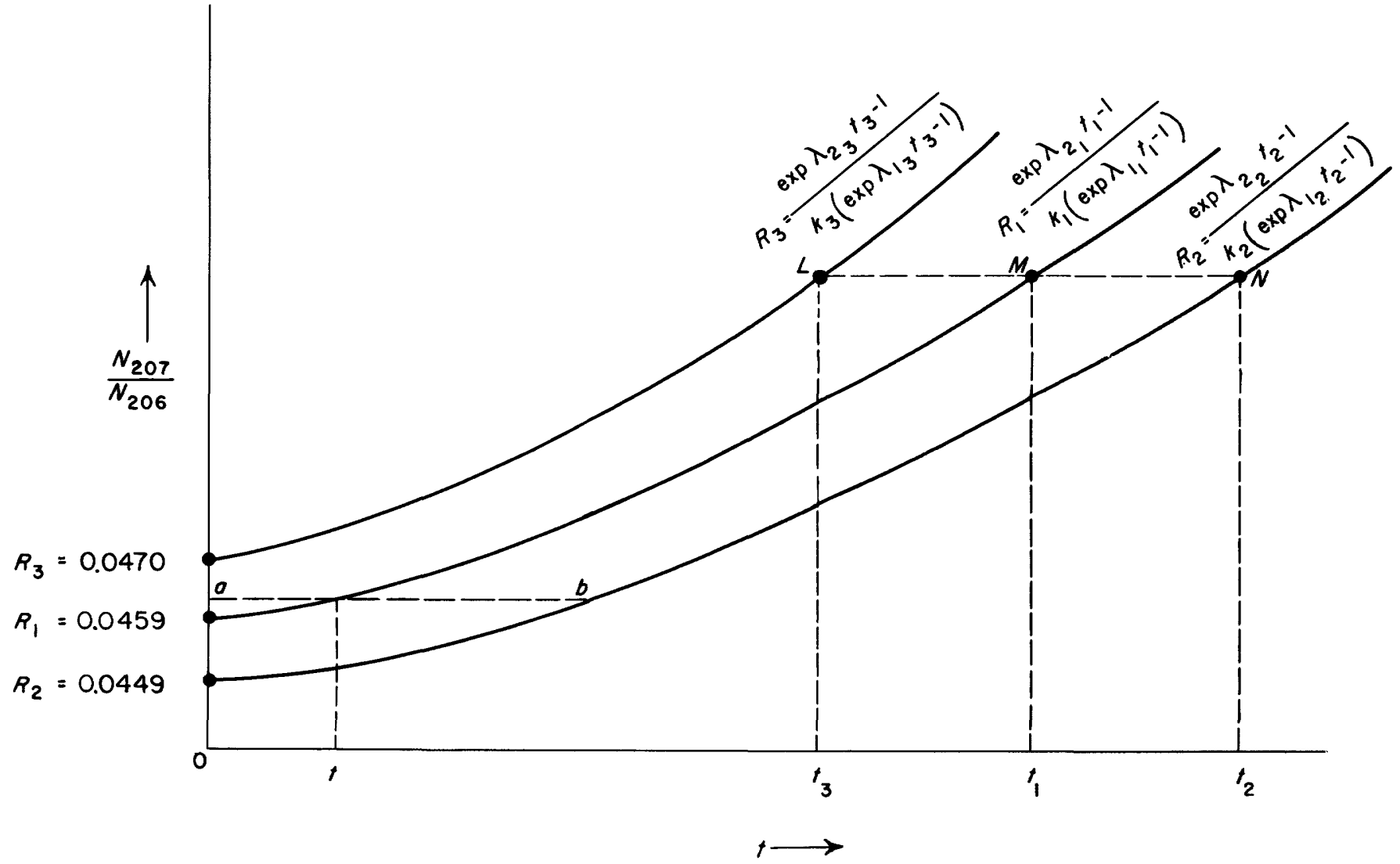


percent. For an exact age solution in terms of numbers of atoms it is not necessary to convert these data from the chemical to physical scale of atomic weights by multiplying these values by the conversion factor 1.0002783 given by Nier (1950). This conversion factor cancels out in the lead-uranium ratio age calculations.

However, for an exact age solution, it is necessary to use the calculated physical atomic weight of the lead based on the isotopic composition of the sample being dated rather than the physical atomic weight of average common lead, 207.282. The physical atomic weight of this hypothetical radiogenic lead is obtained by multiplying the four lead atom percent abundances by the respective physical atomic weights for the individual lead isotopes. Using the isotopic atomic masses given by Sullivan (1957), 204.0368, 206.039, 207.041, and 208.041 , the calculated atomic weight of the radiogenic lead in the uraninite is 206.32. The weight percent of lead corrected for this small factor is:

$$
7.53_{2} \times \frac{207.282}{206.32}=7.56_{7} .
$$

Failure to correct for differences in atomic weight will result in errors of approximately 0.2 to 0.5 percent in the calculated lead-uranium and lead-thorium ages depending on the atomic weight of the lead in the radioactive mineral and the physical constants used in the calculation.

Isotopic analyses, in atom percent, of lead in uraninite and associated hypothetical galena

\begin{tabular}{l|c|c}
\hline & \multicolumn{2}{|c}{ Lead in- } \\
\cline { 2 - 3 } & Uraninite & $\begin{array}{c}\text { Associated } \\
\text { hypothetlcal } \\
\text { galena }\end{array}$ \\
\hline $\mathrm{Pb}^{204}$ & $\mathbf{0 . 1 9 5 _ { 7 }}$ & $\mathbf{1 . 4 3 _ { 6 }}$ \\
$\mathrm{Pb}^{206}$ & $\mathbf{8 1 . 1 7 _ { 8 }}$ & $\mathbf{2 3 . 3 0 _ { 6 }}$ \\
$\mathrm{Pb}^{207}$ & $\mathbf{8 . 6 4 _ { 2 }}$ & $\mathbf{2 3 . 2 7 _ { 7 }}$ \\
$\mathrm{Pb}^{208}$ & $\mathbf{9 . 9 8 _ { 4 }}$ & $\mathbf{5 1 . 9 8 _ { 1 }}$ \\
\hline
\end{tabular}

The presence of lead originally deposited (common lead) with the uraninite and not produced by the radioactive decay of the uranium or thorium in the mineral is indicated by $0.195_{7}$ percent $\mathrm{Pb}^{204}$ in the isotopic analysis of the lead extracted from the uraninite. $\mathrm{Pb}^{204}$ is the only isotope of lead not known to be produced by radioactive decay. The isotopic analysis of the hypothetical galena associated with the uraninite is assumed to approximate closely the isotopic composition of the nonradiogenic lead originally deposited with the uraninite. As this galena also contains $\mathrm{Pb}^{206}$,
$\mathrm{Pb}^{207}$, and $\mathrm{Pb}^{208}$, it is necessary to correct the uraninite lead before the age calculations can be made.

\section{CORRECTION FOR ORIGINAL COMMON LEAD}

The correction for the original common lead may be made in the following way:

1. Using $\mathrm{Pb}^{204}$ as the "index" of the amount of original lead present, a factor proportional to the amount of $\mathrm{Pb}^{204}$ present in the uraninite $\left(0.195_{7}\right)$ compared to the $\mathrm{Pb}^{204}$ in the galena $\left(1.43_{6}\right)$ is obtained.

$$
\frac{0.195_{7}}{1.43_{6}}=0.1362_{8}
$$

2. This factor, when multiplied by the percent abundances of the lead isotopes in the associated galena, will give the proportional amounts of $\mathrm{Pb}^{204}$, $\mathrm{Pb}^{206}, \mathrm{~Pb}^{207}$, and $\mathrm{Pb}^{208}$ originally present in the uraninite.

$$
\begin{aligned}
& \mathrm{Pb}^{204}=1.43_{6} \times 0.1362_{8}=0.195_{7} \\
& \mathrm{~Pb}^{206}=23.30_{6} \times 0.1362_{8}=3.17_{6} \\
& \mathrm{~Pb}^{207}=23.27_{7} \times 0.1362_{8}=3.17_{2} \\
& \mathrm{~Pb}^{208}=51.98_{1} \times 0.1362_{8}=7.08_{4}
\end{aligned}
$$

\begin{tabular}{|c|c|c|c|c|}
\hline & $\mathrm{Pb}^{204}$ & $\mathbf{P b}^{200}$ & $F^{9} b^{907}$ & $\mathrm{~Pb}^{200}$ \\
\hline $\begin{array}{l}\text { Isotopic analysis of uran- } \\
\text { inite lead }\end{array}$ & o. $195_{7}$ & $81.17 \mathrm{~g}$ & E. $64_{2}$ & 9. $98_{4}$ \\
\hline $\begin{array}{l}\text { Isotopic analysis of origi- } \\
\text { nal lead present }\end{array}$ & $-.195_{7}$ & -3.17 & $-\varepsilon .17_{2}$ & -7.08 \\
\hline $\begin{array}{l}\text { Radiogenic lead pro- } \\
\text { duced by } \mathrm{U}+\mathrm{Th}\end{array}$ & 0.000 & 78. $\mathrm{OO}_{2}$ & ह. 470 & 2. $90_{0}$ \\
\hline
\end{tabular}

3. The isotopic analysis of the lead, in atom percent, from the uraninite is then corrected for the original lead present.

\section{$\mathbf{P b}^{200 / \text { T238 AGE METHOD }}$}

The $\mathrm{Pb}^{206} / \mathrm{U}^{238}$ age is obtained by first multiplying the weight percent (chemical scale) of totel lead by the corrected percent of radiogenic $\mathrm{Pb}^{206}$ produced by the uranium and dividing by the weight perc ont (chemical scale) of total uranium multiplied by the atom percent abundance of $\mathrm{U}^{238}$, that is $7.56_{7} \times 78.00_{2}$ divided by $43.6_{46} \times 99.27_{3}$. It is then necessary to convert this weight ratio into an atomic ratio by dividing the physical atomic weight of the uranium, $2 £ \$ .103$, by the physical atomic weight of the lead, 206.32; that is,

$$
\frac{238.103}{206.32}=1.154_{0} \text {. }
$$

As Avagadro's number would appear in both the denominator and numerator, it can be canceled out 
without altering the numerical value of the ratio $N_{d} / N_{p}$. The age calculation thus becomes

$$
N_{200} / N_{238}=\frac{7.56_{7} \times 78.00_{2}}{43.6_{46} \times 99.27_{3}} \times 1.154_{0}=0.1572_{0} .
$$

From the tables read $950 \pm 2.1$ million years.

\section{$\mathrm{Pb}^{207} / \mathrm{U}^{236}$ AGE METHOD}

Similarly, the $\mathrm{Pb}^{207} / \mathrm{U}^{235}$ age is obtained by multiplying the chemical weight percent of total lead by the corrected atom percent abundance of radiogenic $\mathrm{Pb}^{207}$ and dividing by the chemical weight percent of total uranium multiplied by the atom percent abundance of $\mathrm{U}^{235}$. The conversion factor of weight to atom percent, $1.154_{0}$, is used to change the lead-uranium ratio to an atomic ratio. The $\mathrm{Pb}^{207} / \mathrm{U}^{235}$ age thus becomes

$$
N_{207} / N_{235}=\frac{7.56_{7} \times 5.47_{0}}{43.6_{48} \times 0.720_{9}} \times 1.154_{0}=1.518_{1} .
$$

From the tables read $950 \pm 21.3$ million years.

\section{$\mathbf{P b}^{207} / \mathbf{P b}^{208}$ AGE METHOD}

The age calculated from the $\mathrm{Pb}^{207} / \mathrm{Pb}^{206}$ ratio may be obtained directly from the isotopic composition of the remaining radiogenic $\mathrm{Pb}^{206}$ and $\mathrm{Pb}^{207}$. The $\mathrm{Pb}^{207} / \mathrm{Pb}^{206}$ age thus becomes

$$
N_{207} / N_{208}=\frac{5.47_{0}}{78.00_{2}}=0.0701_{3} .
$$

From the tables read $950 \pm 71.9$ million years.

\section{$\mathbf{P b}^{208} / \mathrm{Th}^{232}$ AGE METHOD}

The $\mathrm{Pb}^{208} / \mathrm{Th}^{232}$ age is obtained by multiplying the total chemical weight percent of lead by the atom percent abundance of remaining radiogenic $\mathrm{Pb}^{208}$ and dividing by the total chemical weight percent of thorium times 100. This ratio is converted to an atomic ratio by using the following factor:

$$
\frac{232.111}{206.32}=1.1250_{0} \text {. }
$$

The $\mathrm{Pb}^{208} / \mathrm{Th}^{232}$ age thus becomes

$$
N_{208} / N_{232}=\frac{7.56_{7} \times 2.90_{0}}{5.20_{1} \times 100} \times 1.125_{0}=0.0474_{7} .
$$

From the tables read $950 \pm 46.8$ million years.

Physical isotopic masses were calculated from data given by Huizenga (1955) and by Sullivan (1957).

\section{LITERATURE CITED}

Fleming, E. H., Jr., Ghiorso, A., and Cunningham, B. B., 1952, The specific alpha-activities and half-lives of $\mathrm{U}^{234}, \mathrm{U}^{23^{\circ}}$, and U236: Phys. Rev., v. 88, p. 642-652.

Holmes, Arthur, 1931, Radioactivity and geological time, pt. 4 of The age of the earth: Natl. Research Council Bull. 80, p. 124-459.

Huizenga, J. R., 1955, Isotopic masses $A>201$ : Physica, v. 21, p. 410-424.

Keevil, N. B., 1939, The calculation of geological age: Am. Jour. Sci., v. 237, p. 195-214.

Kovarik, A. F., 1931, Calculating the age of minerals from radioactivity data and principles, pt. 3 of The age of the earth: Natl. Research Council Bull. 80, p. 73-123.

Kovarik, A. F. and Adams, N. I., Jr., 1938, The disintegration constant of thorium and the branching ratio of thorium $\mathrm{C}$ : Phys. Rev., v. 54, p. 413-421.

- 1955 , Redetermination of the disintegration constant of U238: Phys. Rev., v. 98, p. 46.

Kulp, J. L., Bate, G. L., and Broecker, W. S., 1954, Present status of the lead method of age determination: Am. Jour. Sci., v. 252, p. $345 \cdot 365$.

Nier, A. O., 1950, A redetermination of the relative abunciances of the isotopes of carbon, nitrogen, oxygen, argon, and potassium: Phys. Rev., v. 77, p. 789.

Rosholt, J. N., 1958, Radioactive disequilibrium studies as an aid in understanding the natural migration of uranium and its decay products: United Nations Internat. Conf. Penceful Uses of Atomic Energy, 2d, Geneva, 1958, Proc. v. 2, Survey of raw material resources, p. 230-236.

Senftle, F. E., Farley, T. A., and Lazar, N., 1956, Half-life of $\mathrm{Th}^{232}$ and the branching ratio of $\mathrm{Bi}^{212}$ : Phys. Rev., v. 104, p. 1629.

Senftle, F. E., Stieff, L. R., Cuttitta, Frank, and Kuroda, P. K., 1957, Comparison of the isotopic abundance of $\mathrm{U}^{235}$ and $\mathrm{U}^{238}$ and the radium activity ratios in Colorado Plateau uranium ores: Geochim. et Cosmochim. Acta, v. 11, p. 189-193.

Sullivan, W. H., 1957, Trilinear chart of nuclides: U. S. Government Printing Office, Washington 25, D. C.

Wickman, F. E., 1939, Some graphs on the calculation of geological age: Sveriges Geol. Undersökning Årsbok, v. 33, no. 7 , p. 1-8. 



\section{TABLES FOR THE GALCULATION OF LEAD ISOTOPE AGES}

[Numbers above tables are the ages, in millions of years, that are given on each page] 


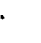


Geologic age, in millions of years, calculated from the atomic ratios of indicated isotopes

\begin{tabular}{|c|c|c|c|c|c|c|c|c|c|c|c|}
\hline \multicolumn{3}{|c|}{$N_{206} / N_{238}$} & \multicolumn{3}{|c|}{$N_{207} / N_{235}$} & \multicolumn{3}{|c|}{$N_{207} / N_{206}$} & \multicolumn{3}{|c|}{$N_{208} / N_{232}$} \\
\hline \multirow[b]{2}{*}{ Ratio } & \multicolumn{2}{|c|}{ Age } & \multirow[b]{2}{*}{ Ratio } & \multicolumn{2}{|c|}{ Age } & \multirow[b]{2}{*}{ Ratio } & \multicolumn{2}{|c|}{ Age } & \multirow[b]{2}{*}{ Ratio } & \multicolumn{2}{|c|}{ Age } \\
\hline & $\begin{array}{c}\text { Number } \\
\text { of } \\
\text { years }\end{array}$ & $\begin{array}{c}\text { Error } \\
\pm\end{array}$ & & \begin{tabular}{|c|}
$\begin{array}{c}\text { Number } \\
\text { of } \\
\text { years }\end{array}$ \\
\end{tabular} & $\begin{array}{c}\text { Error } \\
\pm\end{array}$ & & \begin{tabular}{|c|}
$\begin{array}{c}\text { Number } \\
\text { of } \\
\text { years }\end{array}$ \\
\end{tabular} & $\begin{array}{c}\text { Error } \\
\pm\end{array}$ & & $\begin{array}{c}\text { Number } \\
\text { of } \\
\text { years }\end{array}$ & $\begin{array}{c}\text { Error } \\
\pm\end{array}$ \\
\hline $\begin{array}{l}0.000153 \\
0.000307 \\
0.000461 \\
0.000614 \\
0.000768\end{array}$ & $\begin{array}{l}1 \\
2 \\
3 \\
4 \\
5\end{array}$ & $\begin{array}{l}0.0 \\
0.0 \\
0.0 \\
0.0 \\
0.0\end{array}$ & $\begin{array}{l}0.000972 \\
0.001945 \\
0.002920 \\
0.003895 \\
0.004871\end{array}$ & $\begin{array}{l}1 \\
2 \\
3 \\
4 \\
5\end{array}$ & $\begin{array}{l}0.0 \\
0.0 \\
0.1 \\
0.1 \\
0.1\end{array}$ & $\begin{array}{l}0.045955 \\
0.045974 \\
0.045993 \\
0.046012 \\
0.046030\end{array}$ & $\begin{array}{l}1 \\
2 \\
3 \\
4 \\
5\end{array}$ & $\begin{array}{l}55.8 \\
55.2 \\
55.2 \\
55.2 \\
55.1\end{array}$ & $\begin{array}{l}0.000048 \\
0.000097 \\
0.000146 \\
0.000195 \\
0.000244\end{array}$ & $\begin{array}{l}1 \\
2 \\
3 \\
4 \\
5\end{array}$ & $\begin{array}{l}0.0 \\
0.1 \\
0.1 \\
0.2 \\
0.2\end{array}$ \\
\hline $\begin{array}{l}0.000922 \\
0.001075 \\
0.001229 \\
0.001383 \\
0.001537\end{array}$ & $\begin{array}{r}6 \\
7 \\
8 \\
9 \\
10\end{array}$ & $\begin{array}{l}0.0 \\
0.0 \\
0.0 \\
0.0 \\
0.0\end{array}$ & $\begin{array}{l}0.005849 \\
0.006828 \\
0.007807 \\
0.008787 \\
0.009768\end{array}$ & $\begin{array}{r}6 \\
7 \\
8 \\
9 \\
10\end{array}$ & $\begin{array}{l}0.1 \\
0.2 \\
0.2 \\
0.2 \\
0.2\end{array}$ & $\begin{array}{l}0.046049 \\
0.046068 \\
0.046087 \\
0.046106 \\
0.046124\end{array}$ & $\begin{array}{r}6 \\
7 \\
8 \\
9 \\
10\end{array}$ & $\begin{array}{l}55.1 \\
55.1 \\
55.1 \\
55.1 \\
55.1\end{array}$ & $\begin{array}{l}0.000292 \\
0.000341 \\
0.000390 \\
0.000439 \\
0.000488\end{array}$ & $\begin{array}{r}6 \\
7 \\
8 \\
9 \\
10\end{array}$ & $\begin{array}{l}0.3 \\
0.3 \\
0.4 \\
0.4 \\
0.5\end{array}$ \\
\hline $\begin{array}{l}0.001691 \\
0.001845 \\
0.001998 \\
0.002153 \\
0.002307\end{array}$ & $\begin{array}{l}11 \\
12 \\
13 \\
14 \\
15\end{array}$ & $\begin{array}{l}0.0 \\
0.0 \\
0.0 \\
0.0 \\
0.0\end{array}$ & $\begin{array}{l}0.010750 \\
0.011733 \\
0.012717 \\
0.013702 \\
0.014688\end{array}$ & $\begin{array}{l}11 \\
12 \\
13 \\
14 \\
15\end{array}$ & $\begin{array}{l}0.2 \\
0.3 \\
0.3 \\
0.3\end{array}$ & $\begin{array}{l}0.046143 \\
0.046162 \\
0.046181 \\
0.046200 \\
0.046219\end{array}$ & $\begin{array}{l}11 \\
12 \\
13 \\
14 \\
15\end{array}$ & $\begin{array}{l}56.6 \\
56.7 \\
57.7 \\
56.7\end{array}$ & $\begin{array}{l}0.000536 \\
0.000585 \\
0.000634 \\
0.000683 \\
0.000732\end{array}$ & $\begin{array}{l}11 \\
12 \\
13 \\
14 \\
15\end{array}$ & $\begin{array}{l}0.5 \\
0.6 \\
0.6 \\
0.7 \\
0.7\end{array}$ \\
\hline $\begin{array}{l}0.002462 \\
0.002615 \\
0.002769 \\
0.002924 \\
0.003077\end{array}$ & $\begin{array}{l}16 \\
17 \\
18 \\
19 \\
20\end{array}$ & $\begin{array}{l}0.0 \\
0.0 \\
0.0 \\
0.0 \\
0.0\end{array}$ & $\begin{array}{l}0.015674 \\
0.016662 \\
0.017651 \\
0.018642 \\
0.019633\end{array}$ & $\begin{array}{l}16 \\
17 \\
18 \\
19 \\
20\end{array}$ & $\begin{array}{l}0.4 \\
0.4 \\
0.4 \\
0.4\end{array}$ & $\begin{array}{l}0.046237 \\
0,046256 \\
0.046275 \\
0.046294 \\
0.046313\end{array}$ & $\begin{array}{l}16 \\
17 \\
18 \\
19 \\
20\end{array}$ & $\begin{array}{l}56.5 \\
56.6\end{array}$ & $\begin{array}{l}0.000781 \\
0.000829 \\
0.000878 \\
0.000927 \\
0.000976\end{array}$ & $\begin{array}{l}16 \\
17 \\
18 \\
19 \\
20\end{array}$ & $\begin{array}{l}0.8 \\
0.8 \\
0.9 \\
0.9 \\
1.0\end{array}$ \\
\hline $\begin{array}{l}0.003232 \\
0.003386 \\
0.003540 \\
0.003694 \\
0.003849\end{array}$ & $\begin{array}{l}21 \\
22 \\
23 \\
24 \\
25\end{array}$ & $\begin{array}{l}0.0 \\
0.0 \\
0.1 \\
0.1 \\
0.1\end{array}$ & $\begin{array}{l}0.020624 \\
0.021616 \\
0.022609 \\
0.023605 \\
0.024600\end{array}$ & $\begin{array}{l}21 \\
22 \\
23 \\
24 \\
25\end{array}$ & $\begin{array}{l}0.5 \\
0.5 \\
0.5 \\
0.5 \\
0.6\end{array}$ & $\begin{array}{l}0.046337 \\
0.046361 \\
0.046380 \\
0.046399 \\
0.046417\end{array}$ & $\begin{array}{l}21 \\
22 \\
23 \\
24 \\
25\end{array}$ & $\begin{array}{l}56.3 \\
56.3 \\
56.4\end{array}$ & $\begin{array}{l}0.001025 \\
0.001073 \\
0.001122 \\
0.001171 \\
0.001220\end{array}$ & $\begin{array}{l}21 \\
22 \\
23 \\
24 \\
25\end{array}$ & $\begin{array}{l}1.0 \\
1.1 \\
1.1 \\
1.2 \\
1.2\end{array}$ \\
\hline $\begin{array}{l}0.004002 \\
0.004157 \\
0.004312 \\
0.004466 \\
0.004620\end{array}$ & $\begin{array}{l}26 \\
27 \\
28 \\
29 \\
30\end{array}$ & $\begin{array}{l}0.1 \\
0.1 \\
0.1 \\
0.1 \\
0.1\end{array}$ & $\begin{array}{l}0.025597 \\
0.026595 \\
0.027593 \\
0.028592 \\
0.029593\end{array}$ & $\begin{array}{l}26 \\
27 \\
28 \\
29 \\
30\end{array}$ & $\begin{array}{l}0.6 \\
0.6 \\
0.6 \\
07\end{array}$ & $\begin{array}{l}0.046436 \\
0.046455 \\
0.046474 \\
0.046493 \\
0.046513\end{array}$ & $\begin{array}{l}26 \\
27 \\
28 \\
29 \\
30\end{array}$ & $\begin{array}{l}56.9 \\
56.4 \\
56.0\end{array}$ & $\begin{array}{l}0.001269 \\
0.001317 \\
0.001366 \\
0.001416 \\
0.001465\end{array}$ & $\begin{array}{l}26 \\
27 \\
28 \\
29 \\
30\end{array}$ & $\begin{array}{l}1.3 \\
1.3 \\
1.4 \\
1.4 \\
1.5\end{array}$ \\
\hline $\begin{array}{l}0,004775 \\
0.004930 \\
0.005083 \\
0.005238 \\
0.005393\end{array}$ & $\begin{array}{l}31 \\
32 \\
33 \\
34 \\
35\end{array}$ & $\begin{array}{l}0.1 \\
0.1 \\
0.1 \\
0.1 \\
0.1\end{array}$ & $\begin{array}{l}0.030594 \\
0.031597 \\
0.032600 \\
0.033605 \\
0.034609\end{array}$ & $\begin{array}{l}31 \\
32 \\
33 \\
34 \\
35\end{array}$ & $\begin{array}{l}0.7 \\
0.7 \\
0.7 \\
0.8 \\
0.8\end{array}$ & $\begin{array}{l}0.046532 \\
0.046551 \\
0.046570 \\
0.046589 \\
0.046607\end{array}$ & $\begin{array}{l}31 \\
32 \\
33 \\
34 \\
35\end{array}$ & $56 ?$ & $\begin{array}{l}0.001514 \\
0.001563 \\
0.001611 \\
0.001660 \\
0.001709\end{array}$ & $\begin{array}{l}31 \\
32 \\
33 \\
34 \\
35\end{array}$ & $\begin{array}{l}1.5 \\
1.6 \\
1.6 \\
1.7 \\
1.7\end{array}$ \\
\hline $\begin{array}{l}0.005547 \\
0.005702 \\
0.005857 \\
0.006010 \\
0.006165\end{array}$ & $\begin{array}{l}36 \\
37 \\
38 \\
39 \\
40\end{array}$ & $\begin{array}{l}0.1 \\
0.1 \\
0.1 \\
0.1 \\
0.1\end{array}$ & $\begin{array}{l}0.035616 \\
0.036622 \\
0.037632 \\
0.038641 \\
0.039651\end{array}$ & $\begin{array}{l}36 \\
37 \\
38 \\
39 \\
40\end{array}$ & $\begin{array}{l}0.8 \\
0.8 \\
0.9 \\
0.9 \\
0.9\end{array}$ & $\begin{array}{l}0.046626 \\
0.046645 \\
0.046664 \\
0.046682 \\
0.046701\end{array}$ & $\begin{array}{l}36 \\
37 \\
38 \\
39 \\
40\end{array}$ & $\begin{array}{l}56.4 \\
56.2 \\
56.1 \\
56.8 \\
56.5\end{array}$ & $\begin{array}{l}0.001758 \\
0.001807 \\
0.001855 \\
0.001904 \\
0.001953\end{array}$ & $\begin{array}{l}36 \\
37 \\
38 \\
39 \\
40\end{array}$ & $\begin{array}{l}1.8 \\
1.8 \\
1.9 \\
1.9 \\
2.0\end{array}$ \\
\hline $\begin{array}{l}0.006320 \\
0.006475 \\
0.006629 \\
0.006784 \\
0.006939\end{array}$ & $\begin{array}{l}41 \\
42 \\
43 \\
44 \\
45\end{array}$ & $\begin{array}{l}0.1 \\
0.1 \\
0.1 \\
0.1 \\
0.1\end{array}$ & $\begin{array}{l}0.040662 \\
0.041674 \\
0.042687 \\
0.043702 \\
0.044716\end{array}$ & $\begin{array}{l}41 \\
42 \\
43 \\
44 \\
45\end{array}$ & $\begin{array}{l}0.9 \\
0.9 \\
1.0 \\
1.0 \\
1.0\end{array}$ & $\begin{array}{l}0.046720 \\
0.046739 \\
0.046758 \\
0.046777 \\
0.046796\end{array}$ & $\begin{array}{l}41 \\
42 \\
43 \\
44 \\
45\end{array}$ & $\begin{array}{l}56.4 \\
56.3 \\
56.6 \\
56.5 \\
56.3\end{array}$ & $\begin{array}{l}0.002003 \\
0.002052 \\
0.002100 \\
0.002149 \\
0.002198\end{array}$ & $\begin{array}{l}41 \\
42 \\
43 \\
44 \\
45\end{array}$ & $\begin{array}{l}2.0 \\
2.1 \\
2.1 \\
2.2 \\
2.2\end{array}$ \\
\hline $\begin{array}{l}0.007093 \\
0.007249 \\
0.007404 \\
0.007558 \\
0.007713\end{array}$ & $\begin{array}{l}46 \\
47 \\
48 \\
49 \\
50\end{array}$ & $\begin{array}{l}0.1 \\
0.1 \\
0.1 \\
0.1 \\
0.1\end{array}$ & $\begin{array}{l}0.045732 \\
0.046749 \\
0.047767 \\
0.048787 \\
0.049807\end{array}$ & $\begin{array}{l}46 \\
47 \\
48 \\
49 \\
50\end{array}$ & $\begin{array}{l}1.0 \\
1.1 \\
1.1 \\
1.1 \\
1.1\end{array}$ & $\begin{array}{l}0,046815 \\
0.046834 \\
0.046853 \\
0.046872 \\
0.046891\end{array}$ & $\begin{array}{l}46 \\
47 \\
48 \\
49 \\
50\end{array}$ & $\begin{array}{l}56.6 \\
56.2 \\
56.1 \\
56.5 \\
56.5\end{array}$ & $\begin{array}{l}0.002247 \\
0.002296 \\
0.002345 \\
0.002393 \\
0.002442\end{array}$ & $\begin{array}{l}46 \\
47 \\
48 \\
49 \\
50\end{array}$ & $\begin{array}{l}2.3 \\
2.3 \\
2.4 \\
2.4 \\
2.5\end{array}$ \\
\hline
\end{tabular}


Geologic age, in millions of years, calculated from the atomic ratios of indicated isotopes

\begin{tabular}{|c|c|c|c|c|c|c|c|c|c|c|c|}
\hline \multicolumn{3}{|c|}{$N_{206} / N_{238}$} & \multicolumn{3}{|c|}{$N_{207} / N_{235}$} & \multicolumn{3}{|c|}{$N_{207} / N_{206}$} & \multicolumn{3}{|c|}{$N_{208} / N_{232}$} \\
\hline \multirow[b]{2}{*}{ Ratio } & \multicolumn{2}{|c|}{ Age } & \multirow[b]{2}{*}{ Ratio } & \multicolumn{2}{|c|}{ Age } & \multirow[b]{2}{*}{ Ratio } & \multicolumn{2}{|c|}{ Age } & \multirow[b]{2}{*}{ Ratio } & \multicolumn{2}{|c|}{ Age } \\
\hline & $\begin{array}{c}\text { Number } \\
\text { of } \\
\text { years }\end{array}$ & $\begin{array}{c}\text { Error } \\
\pm\end{array}$ & & \begin{tabular}{|c|}
$\begin{array}{c}\text { Number } \\
\text { of } \\
\text { years }\end{array}$ \\
\end{tabular} & $\begin{array}{c}\text { Error } \\
\pm\end{array}$ & & $\begin{array}{c}\text { Number } \\
\text { of } \\
\text { years }\end{array}$ & $\begin{array}{c}\text { Error } \\
\pm \\
\end{array}$ & & $\begin{array}{c}\begin{array}{c}\text { Number } \\
\text { of } \\
\text { years }\end{array} \\
\end{array}$ & $\begin{array}{c}\text { Error } \\
\pm\end{array}$ \\
\hline $\begin{array}{l}0.007868 \\
0.008022 \\
0.008178 \\
0.008333 \\
0.008488\end{array}$ & $\begin{array}{l}51 \\
52 \\
53 \\
54 \\
55\end{array}$ & $\begin{array}{l}0.1 \\
0.1 \\
0.1 \\
0.1 \\
0.1\end{array}$ & $\begin{array}{l}0.050829 \\
0.051850 \\
0.052873 \\
0.053897 \\
0.054922\end{array}$ & $\begin{array}{l}51 \\
52 \\
53 \\
54 \\
55\end{array}$ & $\begin{array}{l}1.1 \\
1.2 \\
1.2 \\
1.2 \\
1.2\end{array}$ & $\begin{array}{l}0.046910 \\
0.046929 \\
0.046948 \\
0.046967 \\
0.046986\end{array}$ & $\begin{array}{l}51 \\
52 \\
53 \\
54 \\
55\end{array}$ & $\begin{array}{l}56.6 \\
56.7 \\
56.4 \\
56.4 \\
56.5\end{array}$ & $\begin{array}{l}0.002492 \\
0.002541 \\
0.002590 \\
0.002638 \\
0.002687\end{array}$ & $\begin{array}{l}51 \\
52 \\
53 \\
54 \\
55\end{array}$ & $\begin{array}{l}2.5 \\
2.6 \\
2.6 \\
2.7 \\
2.7\end{array}$ \\
\hline $\begin{array}{l}0.008643 \\
0.008798 \\
0.008953 \\
0.009108 \\
0.009263\end{array}$ & $\begin{array}{l}56 \\
57 \\
58 \\
59 \\
60\end{array}$ & $\begin{array}{l}0.1 \\
0.1 \\
0.1 \\
0.1 \\
0.1\end{array}$ & $\begin{array}{l}947 \\
976 \\
003 \\
032 \\
063\end{array}$ & $\begin{array}{l}56 \\
57 \\
58 \\
59 \\
60\end{array}$ & $\begin{array}{l}1.3 \\
1.3 \\
1.3 \\
1.3 \\
1.3\end{array}$ & $\begin{array}{l}0.0470_{05} \\
0.0470_{24} \\
0.0470_{43} \\
0.0470_{62} \\
0.047081\end{array}$ & $\begin{array}{l}56 \\
57 \\
58 \\
59 \\
60\end{array}$ & $\begin{array}{l}56 \\
56 \\
56 \\
56 \\
56\end{array}$ & $\begin{array}{l}736 \\
785 \\
835 \\
883 \\
932\end{array}$ & $\begin{array}{l}56 \\
57 \\
58 \\
59 \\
60\end{array}$ & $\begin{array}{l}2 \\
2 \\
3\end{array}$ \\
\hline $\begin{array}{l}0.009418 \\
0.009573 \\
0.009728 \\
0.009884 \\
0.010038\end{array}$ & $\begin{array}{l}61 \\
62 \\
63 \\
64 \\
65\end{array}$ & $\begin{array}{l}0.1 \\
0.1 \\
0.1 \\
0.1 \\
0.1\end{array}$ & $\begin{array}{l}093 \\
125 \\
159 \\
193 \\
228\end{array}$ & $\begin{array}{l}61 \\
62 \\
63 \\
64 \\
65\end{array}$ & $\begin{array}{l}1.4 \\
1.4 \\
1.4 \\
1.4 \\
1.5\end{array}$ & $\begin{array}{l}0.047100 \\
0.047119 \\
0.047138 \\
0.047157 \\
0.047176\end{array}$ & $\begin{array}{l}61 \\
62 \\
63 \\
64 \\
65\end{array}$ & $\begin{array}{l}56.6 \\
56.7 \\
56.8 \\
56.7 \\
56.9\end{array}$ & $\begin{array}{l}981 \\
030 \\
079 \\
128 \\
177\end{array}$ & $\begin{array}{l}61 \\
62 \\
63 \\
64 \\
65\end{array}$ & $\begin{array}{l}3.1 \\
3.1 \\
3.2 \\
3.2\end{array}$ \\
\hline $\begin{array}{l}0.010194 \\
0.010350 \\
0.010505 \\
0.010660 \\
0.010815\end{array}$ & $\begin{array}{l}66 \\
67 \\
68 \\
69 \\
70\end{array}$ & $\begin{array}{l}0.1 \\
0.1 \\
0.2 \\
0.2 \\
0.2\end{array}$ & $\begin{array}{l}264 \\
301 \\
339 \\
378 \\
18\end{array}$ & $\begin{array}{l}66 \\
67 \\
68 \\
69 \\
70\end{array}$ & $\begin{array}{l}1.5 \\
1.5 \\
1.5 \\
1.5 \\
1.6\end{array}$ & $\begin{array}{l}0.047196 \\
0.047216 \\
0.047243 \\
0.047264 \\
0.047284\end{array}$ & $\begin{array}{l}66 \\
67 \\
68 \\
69 \\
70\end{array}$ & $\begin{array}{l}56 \\
56 \\
56 \\
56 \\
56\end{array}$ & $\begin{array}{l}3226 \\
3275 \\
3324 \\
3373 \\
3421\end{array}$ & $\begin{array}{l}66 \\
67 \\
68 \\
69 \\
70\end{array}$ & $\begin{array}{l}3.3 \\
3.4 \\
3.4 \\
3.5\end{array}$ \\
\hline $\begin{array}{l}0.010971 \\
0.011126 \\
0.011281 \\
0.011437 \\
0.011592\end{array}$ & $\begin{array}{l}71 \\
72 \\
73 \\
74 \\
75\end{array}$ & $\begin{array}{l}0.2 \\
0.2 \\
0.2 \\
0.2 \\
0.2\end{array}$ & $\begin{array}{l}59 \\
01 \\
45 \\
89 \\
34\end{array}$ & $\begin{array}{l}71 \\
72 \\
73 \\
74 \\
75\end{array}$ & $\begin{array}{l}1.6 \\
1.6 \\
1.6 \\
1.7 \\
1.7\end{array}$ & $\begin{array}{l}01 \\
22 \\
44 \\
61 \\
83\end{array}$ & $\begin{array}{l}71 \\
72 \\
73 \\
74 \\
75\end{array}$ & & $\begin{array}{l}71 \\
20 \\
69 \\
18 \\
67\end{array}$ & $\begin{array}{l}71 \\
72 \\
73 \\
74 \\
75\end{array}$ & $\begin{array}{l}3.5 \\
3.5 \\
3.6 \\
3.6 \\
3.7\end{array}$ \\
\hline $\begin{array}{l}0.011748 \\
0.011904 \\
0.012058 \\
0.012214 \\
0.012370\end{array}$ & $\begin{array}{l}76 \\
77 \\
78 \\
79 \\
80\end{array}$ & $\begin{array}{l}0.2 \\
0.2 \\
0.2 \\
0.2 \\
0.2\end{array}$ & $\begin{array}{l}81 \\
27 \\
75 \\
25 \\
75\end{array}$ & $\begin{array}{l}76 \\
77 \\
78 \\
79 \\
80\end{array}$ & $\begin{array}{l}1.7 \\
1.7 \\
1.8 \\
1.8 \\
1.8\end{array}$ & $\begin{array}{l}0.047401 \\
0.047418 \\
0.047443 \\
0.047462 \\
0.047479\end{array}$ & $\begin{array}{l}76 \\
77 \\
78 \\
79 \\
80\end{array}$ & & $\begin{array}{l}15 \\
65 \\
14 \\
63 \\
12\end{array}$ & $\begin{array}{l}76 \\
77 \\
78 \\
79 \\
80\end{array}$ & $\begin{array}{l}3.7 \\
3.8 \\
3.8 \\
3.9 \\
3.9\end{array}$ \\
\hline $\begin{array}{l}0.012526 \\
0.012681 \\
0.012837 \\
0.012993 \\
0.013148\end{array}$ & $\begin{array}{l}81 \\
82 \\
83 \\
84\end{array}$ & $\begin{array}{l}0.2 \\
0.2 \\
0.2 \\
0.2\end{array}$ & $\begin{array}{l}26 \\
79 \\
32 \\
86 \\
42\end{array}$ & $\begin{array}{l}81 \\
82 \\
83 \\
84 \\
85\end{array}$ & $\begin{array}{l}1.8 \\
1.8 \\
1.9 \\
1.9\end{array}$ & $\begin{array}{l}98 \\
520 \\
38 \\
57 \\
579\end{array}$ & $\begin{array}{l}81 \\
82 \\
83 \\
84 \\
85\end{array}$ & $\begin{array}{l}56 \\
57 \\
57\end{array}$ & & $\begin{array}{l}81 \\
82 \\
83 \\
84 \\
85\end{array}$ & $\begin{array}{l}4.1 \\
4.1\end{array}$ \\
\hline $\begin{array}{l}0.013304 \\
0.013460 \\
0.013615 \\
0.013771 \\
0.013927\end{array}$ & $\begin{array}{l}86 \\
87 \\
88 \\
89 \\
90\end{array}$ & $\begin{array}{l}0.2 \\
0.2 \\
0.2 \\
0.2 \\
0.2\end{array}$ & $\begin{array}{l}0.087198 \\
0.088255 \\
0.089315 \\
0.090374 \\
0.091434\end{array}$ & $\begin{array}{l}86 \\
87 \\
88 \\
89 \\
90\end{array}$ & $\begin{array}{l}1.9 \\
2.0 \\
2.0\end{array}$ & $\begin{array}{l}0.047598 \\
0.047616 \\
0.047640 \\
0.047658 \\
0.047677\end{array}$ & $\begin{array}{l}86 \\
87 \\
88 \\
89 \\
90\end{array}$ & $\begin{array}{l}57.0 \\
56.9 \\
57.1 \\
57.0 \\
57.1\end{array}$ & $\begin{array}{l}4205 \\
4255 \\
4304 \\
4353 \\
4402\end{array}$ & $\begin{array}{l}86 \\
87 \\
88 \\
89 \\
90\end{array}$ & $\begin{array}{l}4.2 \\
4.3 \\
4.3 \\
4.4 \\
4.4\end{array}$ \\
\hline $\begin{array}{l}0.014082 \\
0.014238 \\
0.014395 \\
0.014551 \\
0.014706\end{array}$ & $\begin{array}{l}91 \\
92 \\
93 \\
94\end{array}$ & $\begin{array}{l}0.2 \\
0.2 \\
0.2 \\
0.2 \\
0.2\end{array}$ & $\begin{array}{l}0.092496 \\
0.093558 \\
0.094621 \\
0.095687 \\
0.096753\end{array}$ & $\begin{array}{l}91 \\
92 \\
93 \\
94 \\
95\end{array}$ & $\begin{array}{l}2.0 \\
2.1 \\
2.1 \\
2.1\end{array}$ & $\begin{array}{l}0.047700 \\
0.047719 \\
0.047735 \\
0.047755 \\
0.047778\end{array}$ & $\begin{array}{l}91 \\
92 \\
93 \\
94 \\
95\end{array}$ & $\begin{array}{l}57 \\
57 \\
57 \\
57 \\
57\end{array}$ & $\begin{array}{l}51 \\
00 \\
49 \\
98 \\
47\end{array}$ & $\begin{array}{l}91 \\
92 \\
93 \\
94 \\
95\end{array}$ & $\begin{array}{l}4.5 \\
4.6\end{array}$ \\
\hline $\begin{array}{l}0.014862 \\
0.015019 \\
0.015174 \\
0.015330 \\
0.015487\end{array}$ & $\begin{array}{r}96 \\
97 \\
98 \\
99 \\
100\end{array}$ & $\begin{array}{l}0.2 \\
0.2 \\
0.2 \\
0.2\end{array}$ & $\begin{array}{l}0.097819 \\
0.098887 \\
0.099956 \\
0.101025 \\
0.102096\end{array}$ & $\begin{array}{r}96 \\
97 \\
98 \\
99 \\
100\end{array}$ & \begin{tabular}{l|}
2.2 \\
2.2 \\
2.2 \\
2.2 \\
2.2
\end{tabular} & $\begin{array}{l}0.047798 \\
0.047815 \\
0.047838 \\
0.047857 \\
0.047874\end{array}$ & $\begin{array}{r}96 \\
97 \\
98 \\
99 \\
100\end{array}$ & $\begin{array}{l}57.3 \\
57.2 \\
57.3 \\
57.3 \\
57.2\end{array}$ & $\begin{array}{l}0.004696 \\
0.004745 \\
0.004794 \\
0.004843 \\
0.004892\end{array}$ & $\begin{array}{r}96 \\
97 \\
98 \\
99 \\
100\end{array}$ & $\begin{array}{l}4.7 \\
4.8 \\
4.8 \\
4.9 \\
4.9\end{array}$ \\
\hline
\end{tabular}


$101-150$

Geologic age, in millions of years, calculated from the atomic ratios of indicated isotopes

\begin{tabular}{|c|c|c|c|c|c|c|c|c|c|c|c|}
\hline \multicolumn{3}{|c|}{$N_{206} / N_{238}$} & \multicolumn{3}{|c|}{$N_{207} / N_{235}$} & \multicolumn{3}{|c|}{$N_{207} / N_{206}$} & \multicolumn{3}{|c|}{$N_{208} / N_{232}$} \\
\hline \multirow[b]{2}{*}{ Ratio } & \multicolumn{2}{|c|}{ Age } & \multirow[b]{2}{*}{ Ratio } & \multicolumn{2}{|c|}{ Age } & \multirow[b]{2}{*}{ Ratio } & \multicolumn{2}{|c|}{ Age } & \multirow[b]{2}{*}{ Ratio } & \multicolumn{2}{|c|}{ Age } \\
\hline & \begin{tabular}{|c|}
$\begin{array}{c}\text { Number } \\
\text { of } \\
\text { years }\end{array}$ \\
\end{tabular} & $\begin{array}{c}\text { Error } \\
\pm\end{array}$ & & \begin{tabular}{|c|}
$\begin{array}{c}\text { Number } \\
\text { of } \\
\text { years }\end{array}$ \\
\end{tabular} & $\begin{array}{c}\text { Error } \\
\pm\end{array}$ & & \begin{tabular}{|c|}
$\begin{array}{c}\text { Number } \\
\text { of } \\
\text { years }\end{array}$ \\
\end{tabular} & $\begin{array}{c}\text { Error } \\
\pm\end{array}$ & & \begin{tabular}{|c|}
$\begin{array}{c}\text { Number } \\
\text { of } \\
\text { years }\end{array}$ \\
\end{tabular} & $\begin{array}{c}\text { Error } \\
\pm\end{array}$ \\
\hline $\begin{array}{l}0.015642 \\
0.015798 \\
0.015955 \\
0.016110 \\
0.016267\end{array}$ & $\begin{array}{l}101 \\
102 \\
103 \\
104 \\
105\end{array}$ & $\begin{array}{l}0.2 \\
0.2 \\
0.2 \\
0.2 \\
0.2\end{array}$ & $\begin{array}{l}0.103168 \\
0.104242 \\
0.105316 \\
0.106391 \\
0.107466\end{array}$ & $\begin{array}{l}101 \\
102 \\
103 \\
104 \\
105\end{array}$ & $\begin{array}{l}2.3 \\
2.3 \\
2.3 \\
2.3 \\
2.4\end{array}$ & $\begin{array}{l}0.047898 \\
0.047918 \\
0.047936 \\
0.047959 \\
0.047976\end{array}$ & $\begin{array}{l}101 \\
102 \\
103 \\
104 \\
105\end{array}$ & $\begin{array}{l}57.4 \\
57.5 \\
57.3 \\
57.5 \\
57.3\end{array}$ & $\begin{array}{l}0.004942 \\
0.004990 \\
0.005039 \\
0.005088 \\
0.005138\end{array}$ & $\begin{array}{l}101 \\
102 \\
103 \\
104 \\
105\end{array}$ & $\begin{array}{l}5.0 \\
5.0 \\
5.1 \\
5.1 \\
5.2\end{array}$ \\
\hline $\begin{array}{l}0.016423 \\
0.016580 \\
0.016735 \\
0.016892 \\
0.017048\end{array}$ & $\begin{array}{l}106 \\
107 \\
108 \\
109 \\
110\end{array}$ & $\begin{array}{l}0.2 \\
0.2 \\
0.2 \\
0.2 \\
0.2\end{array}$ & $\begin{array}{l}0.108543 \\
0.109622 \\
0.110701 \\
0.111782 \\
0.112862\end{array}$ & $\begin{array}{l}106 \\
107 \\
108 \\
109 \\
110\end{array}$ & $\begin{array}{l}2.4 \\
2.4 \\
2.4 \\
2.4 \\
2.5\end{array}$ & $\begin{array}{l}0.047997 \\
0.048015 \\
0.048038 \\
0.048057 \\
0.048077\end{array}$ & $\begin{array}{l}106 \\
107 \\
108 \\
109 \\
110\end{array}$ & $\begin{array}{l}57.4 \\
57.3 \\
57.5 \\
57.4 \\
57.4\end{array}$ & $\begin{array}{l}0.005187 \\
0.005236 \\
0.005284 \\
0.005334 \\
0.005383\end{array}$ & $\begin{array}{l}106 \\
107 \\
108 \\
109 \\
110\end{array}$ & $\begin{array}{l}5.2 \\
5.3 \\
5.3 \\
5.4 \\
5.4\end{array}$ \\
\hline $\begin{array}{l}0.017204 \\
0.017361 \\
0.017517 \\
0.017673 \\
0.017830\end{array}$ & $\begin{array}{l}111 \\
112 \\
113 \\
114 \\
115\end{array}$ & $\begin{array}{l}0.2 \\
0.2 \\
0.3 \\
0.3 \\
0.3\end{array}$ & $\begin{array}{l}0.113945 \\
0.115028 \\
0.116113 \\
0.117199 \\
0.118285\end{array}$ & $\begin{array}{l}111 \\
112 \\
113 \\
114 \\
115\end{array}$ & $\begin{array}{l}2.5 \\
2.5 \\
2.5 \\
2.6 \\
2.6\end{array}$ & $\begin{array}{l}0.048098 \\
0.048116 \\
0.048137 \\
0.048159 \\
0.048177\end{array}$ & $\begin{array}{l}111 \\
112 \\
113 \\
114 \\
115\end{array}$ & $\begin{array}{l}57.5 \\
57.4 \\
57.5 \\
57.7 \\
57.6\end{array}$ & $\begin{array}{l}0.005432 \\
0.005481 \\
0.005530 \\
0.005579 \\
0.005628\end{array}$ & $\begin{array}{l}111 \\
112 \\
113 \\
114 \\
115\end{array}$ & $\begin{array}{l}5.5 \\
5.5 \\
5.6 \\
5.6 \\
5.7\end{array}$ \\
\hline $\begin{array}{l}0,017986 \\
0.018142 \\
0.018299 \\
0.018457 \\
0.018614\end{array}$ & $\begin{array}{l}116 \\
117 \\
118 \\
119 \\
120\end{array}$ & $\begin{array}{l}0.3 \\
0.3 \\
0.3 \\
0.3 \\
0.3\end{array}$ & $\begin{array}{l}0.119372 \\
0.120461 \\
0.121551 \\
0.122643 \\
0.123734\end{array}$ & $\begin{array}{l}116 \\
117 \\
118 \\
119 \\
120\end{array}$ & $\begin{array}{l}2.6 \\
2.6 \\
2.6 \\
2.7 \\
2.7\end{array}$ & $\begin{array}{l}0.048198 \\
0.048220 \\
0.048238 \\
0.048255 \\
0.048274\end{array}$ & $\begin{array}{l}116 \\
117 \\
118 \\
119 \\
120\end{array}$ & $\begin{array}{l}57.6 \\
57.7 \\
57.6 \\
57.4 \\
57.4\end{array}$ & $\begin{array}{l}0.005678 \\
0.005727 \\
0.005775 \\
0.005824 \\
0.005874\end{array}$ & $\begin{array}{l}116 \\
117 \\
118 \\
119 \\
120\end{array}$ & $\begin{array}{l}5.7 \\
5.8 \\
5.8 \\
5.9 \\
5.9\end{array}$ \\
\hline $\begin{array}{l}0.018769 \\
0.018926 \\
0.019083 \\
0.019239 \\
0.019396\end{array}$ & $\begin{array}{l}121 \\
122 \\
123 \\
124 \\
125\end{array}$ & $\begin{array}{l}0.3 \\
0.3 \\
0.3 \\
0.3 \\
0.3\end{array}$ & $\begin{array}{l}0.124827 \\
0.125922 \\
0.127016 \\
0128111 \\
0.12921 n\end{array}$ & $\begin{array}{l}121 \\
122 \\
123 \\
124 \\
125\end{array}$ & $\begin{array}{l}2.7 \\
2.7 \\
2.8 \\
2.8 \\
2.8\end{array}$ & $\begin{array}{l}0.048298 \\
0.048317 \\
0.048336 \\
0.048358 \\
0.048378\end{array}$ & $\begin{array}{l}121 \\
122 \\
123 \\
124 \\
125\end{array}$ & $\begin{array}{l}57.6 \\
57.5 \\
57.5 \\
57.6 \\
57.6\end{array}$ & $\begin{array}{l}5923 \\
5972 \\
022 \\
070 \\
5119\end{array}$ & $\begin{array}{l}121 \\
122 \\
123 \\
124 \\
125\end{array}$ & $\begin{array}{l}6.0 \\
6.0 \\
6.1 \\
6.1 \\
6.2\end{array}$ \\
\hline $\begin{array}{l}0.019553 \\
0.019709 \\
0.019866 \\
0.020023 \\
0.020179\end{array}$ & $\begin{array}{l}126 \\
127 \\
128 \\
129 \\
130\end{array}$ & $\begin{array}{l}0.3 \\
0.3 \\
0.3 \\
0.3 \\
0.3\end{array}$ & $\begin{array}{l}0.130309 \\
0.131407 \\
0.132508 \\
0.133609 \\
0.134711\end{array}$ & $\begin{array}{l}126 \\
127 \\
128 \\
129 \\
130\end{array}$ & $\begin{array}{l}2.8 \\
2.8 \\
2.9 \\
2.9 \\
2.9\end{array}$ & $\begin{array}{l}0.048397 \\
0.048419 \\
0.048439 \\
0.048458 \\
0.048480\end{array}$ & $\begin{array}{l}126 \\
127 \\
128 \\
129 \\
130\end{array}$ & $\begin{array}{l}57.6 \\
57.7 \\
57.7 \\
57.6 \\
57.7\end{array}$ & $\begin{array}{l}0.006168 \\
0.006218 \\
0.006267 \\
0.006315 \\
0.006365\end{array}$ & $\begin{array}{l}126 \\
127 \\
128 \\
129 \\
130\end{array}$ & $\begin{array}{l}6.2 \\
6.3 \\
6.3 \\
6.4 \\
6.4\end{array}$ \\
\hline $\begin{array}{l}0.020336 \\
0.020493 \\
0.020650 \\
0.020807 \\
0.020964\end{array}$ & $\begin{array}{l}131 \\
132 \\
133 \\
134 \\
135\end{array}$ & $\begin{array}{l}0.3 \\
0.3 \\
0.3 \\
0.3 \\
0.3\end{array}$ & $\begin{array}{l}0.135815 \\
0.136921 \\
0.138026 \\
0.139133 \\
0.140241\end{array}$ & $\begin{array}{l}131 \\
132 \\
133 \\
134 \\
135\end{array}$ & $\begin{array}{l}2.9 \\
3.0 \\
3.0 \\
3.0 \\
3.0\end{array}$ & $\begin{array}{l}0.048500 \\
0.048521 \\
0.048540 \\
0.048560 \\
0.048581\end{array}$ & $\begin{array}{l}131 \\
132 \\
133 \\
134 \\
135\end{array}$ & $\begin{array}{l}57.8 \\
57.8 \\
57.7 \\
57.8 \\
57.8\end{array}$ & $\begin{array}{l}0.006414 \\
0.006463 \\
0.006513 \\
0.006561 \\
0.006610\end{array}$ & $\begin{array}{l}131 \\
132 \\
133 \\
134 \\
135\end{array}$ & $\begin{array}{l}6.5 \\
6.5 \\
6.6 \\
6.6 \\
6.7\end{array}$ \\
\hline $\begin{array}{l}0.021121 \\
0.021277 \\
0.021434 \\
0.021592 \\
0.021748\end{array}$ & $\begin{array}{l}136 \\
137 \\
138 \\
139 \\
140\end{array}$ & $\begin{array}{l}0.3 \\
0.3 \\
0.3 \\
0.3 \\
0.3\end{array}$ & $\begin{array}{l}0.14135 n \\
0.142460 \\
0.143572 \\
0.144684 \\
0.145797\end{array}$ & $\begin{array}{l}136 \\
137 \\
138 \\
139 \\
140\end{array}$ & $\begin{array}{l}3.1 \\
3.1 \\
3.1 \\
3.1 \\
3.1\end{array}$ & $\begin{array}{l}0.048601 \\
0.048623 \\
0.048644 \\
0.048662 \\
0.048685\end{array}$ & $\begin{array}{l}136 \\
137 \\
138 \\
139 \\
140\end{array}$ & $\begin{array}{l}57.8 \\
57.9 \\
57.9 \\
57.8 \\
58.0\end{array}$ & $\begin{array}{l}0.006660 \\
0.006709 \\
0.006758 \\
0.006808 \\
0.006856\end{array}$ & $\begin{array}{l}136 \\
137 \\
138 \\
139 \\
140\end{array}$ & $\begin{array}{l}6.7 \\
6.8 \\
6.8 \\
69\end{array}$ \\
\hline $\begin{array}{l}0.021905 \\
0.022063 \\
0.022219 \\
0.022376 \\
0.022534\end{array}$ & $\begin{array}{l}141 \\
142 \\
143 \\
144 \\
145\end{array}$ & $\begin{array}{l}0.3 \\
0.3 \\
0.3 \\
0.3 \\
0.3\end{array}$ & $\begin{array}{l}0.146911 \\
0.148027 \\
0.149143 \\
0.150261 \\
0.151380\end{array}$ & $\begin{array}{l}141 \\
142 \\
143 \\
144 \\
145\end{array}$ & $\begin{array}{l}3.2 \\
3.2 \\
3.2 \\
3.2 \\
3.3\end{array}$ & $\begin{array}{l}0.048705 \\
0.048723 \\
0.048746 \\
0.048767 \\
0.048786\end{array}$ & $\begin{array}{l}141 \\
142 \\
143 \\
144 \\
145\end{array}$ & $\begin{array}{l}57.9 \\
57.8 \\
58.0 \\
58.1 \\
58.0\end{array}$ & $\begin{array}{l}0.006905 \\
0.006955 \\
0.007004 \\
0.007053 \\
0.007102\end{array}$ & $\begin{array}{l}141 \\
142 \\
143 \\
144 \\
145\end{array}$ & $\begin{array}{l}7.0 \\
7.0 \\
7.0 \\
7.1 \\
7.1\end{array}$ \\
\hline $\begin{array}{l}0.022691 \\
0.022848 \\
0.023005 \\
0.023163 \\
0.023320\end{array}$ & $\begin{array}{l}146 \\
147 \\
148 \\
149 \\
150\end{array}$ & $\begin{array}{l}0.3 \\
0.3 \\
0.3 \\
0.3 \\
0.3\end{array}$ & $\begin{array}{l}0.152499 \\
0.153621 \\
0.154742 \\
0.155865 \\
0.156989\end{array}$ & $\begin{array}{l}146 \\
147 \\
148 \\
149 \\
150\end{array}$ & $\begin{array}{l}3.3 \\
3.3 \\
3.3 \\
3.3 \\
3.4\end{array}$ & $\begin{array}{l}0.048806 \\
0.048827 \\
0.048848 \\
0.048867 \\
0.048888\end{array}$ & $\begin{array}{l}146 \\
147 \\
148 \\
149 \\
150\end{array}$ & $\begin{array}{l}58.0 \\
58.0 \\
58.1 \\
58.1 \\
58.0\end{array}$ & $\begin{array}{l}0.007151 \\
0.007200 \\
0.007250 \\
0.007299 \\
0.007348\end{array}$ & $\begin{array}{l}146 \\
147 \\
148 \\
149 \\
150\end{array}$ & $\begin{array}{l}7.2 \\
7.2 \\
7.3 \\
7.3 \\
7.4\end{array}$ \\
\hline
\end{tabular}


$151-200$

Geologic age, in millions of years, calculated from the atomic ratios of indicated isotopes

\begin{tabular}{|c|c|c|c|c|c|c|c|c|c|c|c|}
\hline \multicolumn{3}{|c|}{$N_{206} / N_{238}$} & \multicolumn{3}{|c|}{$N_{207} / N_{235}$} & \multicolumn{3}{|c|}{$N_{207} / N_{206}$} & \multicolumn{3}{|c|}{$N_{208} / N_{232}$} \\
\hline \multirow[b]{2}{*}{ Ratio } & \multicolumn{2}{|c|}{ Age } & \multirow[b]{2}{*}{ Ratio } & \multicolumn{2}{|c|}{ Age } & \multirow[b]{2}{*}{ Ratio } & \multicolumn{2}{|c|}{ Age } & \multirow[b]{2}{*}{ Ratio } & \multicolumn{2}{|c|}{ Age } \\
\hline & \begin{tabular}{|c|}
$\begin{array}{c}\text { Number } \\
\text { of } \\
\text { years }\end{array}$ \\
\end{tabular} & $\begin{array}{c}\text { Error } \\
\pm\end{array}$ & & $\begin{array}{c}\text { Number } \\
\text { of } \\
\text { years }\end{array}$ & $\begin{array}{l}\text { Error } \\
\pm\end{array}$ & & \begin{tabular}{|c|}
$\begin{array}{c}\text { Number } \\
\text { of } \\
\text { years }\end{array}$ \\
\end{tabular} & $\begin{array}{c}\text { Error } \\
\pm \\
\end{array}$ & & \begin{tabular}{|c|}
$\begin{array}{c}\text { Number } \\
\text { of } \\
\text { years }\end{array}$ \\
\end{tabular} & $\begin{array}{c}\text { Error } \\
\pm\end{array}$ \\
\hline $\begin{array}{l}0.023478 \\
0.023635 \\
0.023792 \\
0.023950 \\
0.024107\end{array}$ & $\begin{array}{l}151 \\
152 \\
153 \\
154 \\
155\end{array}$ & $\begin{array}{l}0.3 \\
0.3 \\
0.3 \\
0.3 \\
0.3\end{array}$ & $\begin{array}{l}0.158116 \\
0.159241 \\
0.160369 \\
0.161497 \\
0162627\end{array}$ & $\begin{array}{l}151 \\
152 \\
153 \\
154 \\
155\end{array}$ & $\begin{array}{l}3.4 \\
3.4 \\
3.5 \\
3.5\end{array}$ & $\begin{array}{l}0.048908 \\
0.048928 \\
0.048950 \\
0.048969 \\
0.048990\end{array}$ & $\begin{array}{l}151 \\
152 \\
153 \\
154 \\
155\end{array}$ & $\begin{array}{l}58.0 \\
58.0 \\
58.1 \\
58.0 \\
58.1\end{array}$ & $\begin{array}{l}0.007397 \\
0.007446 \\
0.007495 \\
0.007545 \\
0.007594\end{array}$ & $\begin{array}{l}151 \\
152 \\
153 \\
154 \\
155\end{array}$ & $\begin{array}{l}7.4 \\
7.5 \\
7.5 \\
7.6 \\
7.6\end{array}$ \\
\hline $\begin{array}{l}0.024264 \\
0.024422 \\
0.024579 \\
0.024736 \\
0.024894\end{array}$ & $\begin{array}{l}156 \\
157 \\
158 \\
159 \\
160\end{array}$ & $\begin{array}{l}0.3 \\
0.3 \\
0.4 \\
0.4 \\
0.4\end{array}$ & $\begin{array}{l}0.163758 \\
0.164890 \\
0.166023 \\
0.1671 .57 \\
0.168293\end{array}$ & $\begin{array}{l}156 \\
157 \\
158 \\
159 \\
160\end{array}$ & $\begin{array}{l}3.5 \\
3.5 \\
3.5 \\
3.6 \\
3.6\end{array}$ & $\begin{array}{l}0.049012 \\
0.049031 \\
0.049053 \\
0.049075 \\
0.049095\end{array}$ & $\begin{array}{l}156 \\
157 \\
158 \\
159 \\
160\end{array}$ & $\begin{array}{l}58.2 \\
58.1 \\
58.2 \\
58.3 \\
58.2\end{array}$ & $\begin{array}{l}0.007642 \\
0.007692 \\
0.007741 \\
0.007791 \\
0.007840\end{array}$ & $\begin{array}{l}155 \\
157 \\
159 \\
159 \\
1 \leqslant 0\end{array}$ & $\begin{array}{l}7.7 \\
7.7 \\
7.8 \\
7.8 \\
7.9\end{array}$ \\
\hline $\begin{array}{l}0.025052 \\
0.025209 \\
0.025366 \\
0.025524 \\
0.025682\end{array}$ & $\begin{array}{l}161 \\
162 \\
163 \\
164 \\
165\end{array}$ & $\begin{array}{l}0.4 \\
0.4 \\
0.4 \\
0.4 \\
0.4\end{array}$ & $\begin{array}{l}0.169429 \\
0.170566 \\
0.171706 \\
0.172844 \\
0.173985\end{array}$ & $\begin{array}{l}161 \\
162 \\
163 \\
164 \\
165\end{array}$ & $\begin{array}{l}3.6 \\
3.6 \\
3.7 \\
3.7\end{array}$ & $\begin{array}{l}0.049114 \\
0.049136 \\
0.049158 \\
0.049178 \\
0.049198\end{array}$ & $\begin{array}{l}161 \\
162 \\
163 \\
164 \\
165\end{array}$ & $\begin{array}{l}58.2 \\
58.3 \\
58.4 \\
58.3 \\
58.3\end{array}$ & $\begin{array}{l}0.007888 \\
0.007938 \\
0.007987 \\
0.008037 \\
0.008086\end{array}$ & $\begin{array}{l}161 \\
16 ? \\
163 \\
164 \\
165\end{array}$ & $\begin{array}{l}7.9 \\
8.0 \\
8.0 \\
8.1 \\
8.1\end{array}$ \\
\hline $\begin{array}{l}0.025839 \\
0.025997 \\
0.026155 \\
0.026312 \\
0.026470\end{array}$ & $\begin{array}{l}166 \\
167 \\
168 \\
169 \\
170\end{array}$ & $\begin{array}{l}0.4 \\
0.4 \\
0.4 \\
0.4 \\
0.4\end{array}$ & $\begin{array}{l}0.175127 \\
0.176269 \\
0.177414 \\
0.178560 \\
0.179707\end{array}$ & $\begin{array}{l}166 \\
167 \\
168 \\
169 \\
170\end{array}$ & $\begin{array}{l}3.7 \\
3.7 \\
3.8 \\
3.8 \\
3.8\end{array}$ & $\begin{array}{l}0.049220 \\
0.049240 \\
0.049260 \\
0.049282 \\
0.049303\end{array}$ & $\begin{array}{l}166 \\
167 \\
168 \\
169 \\
170\end{array}$ & $\begin{array}{l}58.4 \\
58.3 \\
58.3 \\
58.4 \\
58.5\end{array}$ & $\begin{array}{l}0.008135 \\
0.008184 \\
0.008233 \\
0.008283 \\
0.008332\end{array}$ & $\begin{array}{l}166 \\
167 \\
168 \\
169 \\
170\end{array}$ & $\begin{array}{l}8.2 \\
8.2 \\
8.3 \\
8.3 \\
8.4\end{array}$ \\
\hline $\begin{array}{l}0.026629 \\
0.026786 \\
0.026944 \\
0.027102 \\
0.027260\end{array}$ & $\begin{array}{l}171 \\
172 \\
173 \\
174 \\
175\end{array}$ & $\begin{array}{l}0.4 \\
0.4 \\
0.4 \\
0.4 \\
0.4\end{array}$ & $\begin{array}{l}0.180853 \\
0.182002 \\
0.183151 \\
0.184302 \\
0.185453\end{array}$ & $\begin{array}{l}171 \\
172 \\
173 \\
174 \\
175\end{array}$ & $\begin{array}{l}3.8 \\
3.9 \\
3.9 \\
3.9 \\
3.9\end{array}$ & $\begin{array}{l}0.049321 \\
0.049344 \\
0.049364 \\
0.049384 \\
0.049405\end{array}$ & $\begin{array}{l}171 \\
172 \\
173 \\
174 \\
175\end{array}$ & $\begin{array}{l}58.3 \\
58.4 \\
58.4 \\
58.4 \\
58.4\end{array}$ & $\begin{array}{l}381 \\
430 \\
479 \\
529 \\
578\end{array}$ & $\begin{array}{l}171 \\
172 \\
173 \\
174 \\
175\end{array}$ & $\begin{array}{l}8.4 \\
8.5 \\
8.5 \\
8.6 \\
8.6\end{array}$ \\
\hline $\begin{array}{l}0.027417 \\
0.027576 \\
0.027734 \\
0.027891 \\
0.028049\end{array}$ & $\begin{array}{l}176 \\
177 \\
178 \\
179 \\
180\end{array}$ & $\begin{array}{l}0.4 \\
0.4 \\
0.4 \\
0.4 \\
0.4\end{array}$ & $\begin{array}{l}0.186607 \\
0.187762 \\
0.188917 \\
0.190073 \\
0.191231\end{array}$ & $\begin{array}{l}176 \\
177 \\
178 \\
179 \\
180\end{array}$ & $\begin{array}{l}3.9 \\
4.0 \\
4.0 \\
4.0 \\
4.0\end{array}$ & $\begin{array}{l}0.049428 \\
0.049447 \\
0.049468 \\
0.049490 \\
0.049511\end{array}$ & $\begin{array}{l}176 \\
177 \\
178 \\
179 \\
180\end{array}$ & $\begin{array}{l}58.5 \\
58.4 \\
58.4 \\
58.6 \\
58.6\end{array}$ & $\begin{array}{l}0.008627 \\
0.008676 \\
0.008725 \\
0.008775 \\
0.008824\end{array}$ & $\begin{array}{l}176 \\
177 \\
178 \\
179 \\
180\end{array}$ & $\begin{array}{l}8.7 \\
8.7 \\
8.8 \\
8.8 \\
8.9\end{array}$ \\
\hline $\begin{array}{l}0.028207 \\
0.028365 \\
0.028523 \\
0.028681 \\
0.028839\end{array}$ & $\begin{array}{l}181 \\
182 \\
183 \\
184 \\
185\end{array}$ & $\begin{array}{l}0.4 \\
0.4 \\
0.4 \\
0.4 \\
0.4\end{array}$ & $\begin{array}{l}0.192387 \\
0.193549 \\
0.194710 \\
0.195871 \\
0.197034\end{array}$ & $\begin{array}{l}181 \\
182 \\
183 \\
184 \\
185\end{array}$ & $\begin{array}{l}4.1 \\
4.1 \\
4.1 \\
4.1 \\
4.2\end{array}$ & $\begin{array}{l}0.049531 \\
0.049553 \\
0.049574 \\
0.049595 \\
0.049616\end{array}$ & $\begin{array}{l}181 \\
182 \\
183 \\
184 \\
185\end{array}$ & $\begin{array}{l}58.5 \\
58.6 \\
58.6 \\
58,6 \\
58.6\end{array}$ & $\begin{array}{l}0.008874 \\
0.008923 \\
0.008971 \\
0.009021 \\
0.009070\end{array}$ & $\begin{array}{l}181 \\
182 \\
183 \\
184 \\
185\end{array}$ & $\begin{array}{l}8.9 \\
9.0 \\
9.0 \\
9.1 \\
9.1\end{array}$ \\
\hline $\begin{array}{l}0.028997 \\
0.029155 \\
0.029315 \\
0.029472 \\
0.029631\end{array}$ & $\begin{array}{l}186 \\
187 \\
188 \\
189 \\
190\end{array}$ & $\begin{array}{l}0.4 \\
0.4 \\
0.4 \\
0.4 \\
0.4\end{array}$ & $\begin{array}{l}0.198199 \\
0.199364 \\
0.200531 \\
0.201698 \\
0.202867\end{array}$ & $\begin{array}{l}186 \\
187 \\
188 \\
189 \\
190\end{array}$ & $\begin{array}{l}4.2 \\
4.2 \\
4.2 \\
4.2 \\
4.3\end{array}$ & $\begin{array}{l}0.049638 \\
0.049659 \\
0.049677 \\
0.049700 \\
0.049720\end{array}$ & $\begin{array}{l}186 \\
187 \\
188 \\
189 \\
190\end{array}$ & $\begin{array}{l}58.7 \\
58.7 \\
58.6 \\
58.7 \\
58.7\end{array}$ & $\begin{array}{l}0.009120 \\
0.009169 \\
0.009218 \\
0.009267 \\
0.009317\end{array}$ & $\begin{array}{l}185 \\
187 \\
189 \\
189 \\
180\end{array}$ & $\begin{array}{l}9.2 \\
9.2 \\
9.3 \\
9.3 \\
9.4\end{array}$ \\
\hline $\begin{array}{l}0.029789 \\
0.029947 \\
0.030105 \\
0.030264 \\
0.030422\end{array}$ & $\begin{array}{l}191 \\
192 \\
193 \\
194 \\
195\end{array}$ & $\begin{array}{l}0.4 \\
0.4 \\
0.4 \\
0.4 \\
0.4\end{array}$ & $\begin{array}{l}0.204036 \\
0.205207 \\
0.206379 \\
0.207554 \\
0.208729\end{array}$ & $\begin{array}{l}191 \\
192 \\
193 \\
194 \\
195\end{array}$ & $\begin{array}{l}4.3 \\
4.3 \\
4.3 \\
4.4 \\
4.4\end{array}$ & $\begin{array}{l}0.049741 \\
0.049762 \\
0.049784 \\
0.049804 \\
0.049826\end{array}$ & $\begin{array}{l}191 \\
192 \\
193 \\
194 \\
195\end{array}$ & $\begin{array}{l}58.7 \\
58.7 \\
58.8 \\
58.7 \\
58.8\end{array}$ & $\begin{array}{l}0,009366 \\
0.009415 \\
0.009464 \\
0.009513 \\
0.009563\end{array}$ & $\begin{array}{l}191 \\
192 \\
193 \\
194 \\
195\end{array}$ & $\begin{array}{l}9.4 \\
9.5 \\
9.5 \\
9.6 \\
9.6\end{array}$ \\
\hline $\begin{array}{l}0.030580 \\
0.030739 \\
0.030896 \\
0.031055 \\
0.031214\end{array}$ & $\begin{array}{l}196 \\
197 \\
198 \\
139 \\
200\end{array}$ & $\begin{array}{l}0.4 \\
0.4 \\
0.4 \\
0.4 \\
0.4\end{array}$ & $\begin{array}{l}0.209904 \\
0.211081 \\
0.212259 \\
0.213438 \\
0.214617\end{array}$ & $\begin{array}{l}196 \\
197 \\
198 \\
199 \\
200\end{array}$ & $\begin{array}{l}4.4 \\
4.4 \\
4.4 \\
4.5 \\
4.5\end{array}$ & $\begin{array}{l}0.049848 \\
0.049868 \\
0.049891 \\
0.049912 \\
0.049932\end{array}$ & $\begin{array}{l}196 \\
197 \\
198 \\
199 \\
200\end{array}$ & $\begin{array}{l}58.8 \\
58.8 \\
58.9 \\
58.9 \\
58.8\end{array}$ & $\begin{array}{l}0.009612 \\
0.009662 \\
0.009711 \\
0.009760 \\
0.009809\end{array}$ & $\begin{array}{l}196 \\
197 \\
198 \\
199 \\
200\end{array}$ & $\begin{array}{l}9.7 \\
9.7 \\
9.8 \\
9.8 \\
9.9\end{array}$ \\
\hline
\end{tabular}


Geologic age, in millions of years, calculated from the atomic ratios of indicated isotopes

\begin{tabular}{|c|c|c|c|c|c|c|c|c|c|c|c|}
\hline \multicolumn{3}{|c|}{$N_{206} / N_{238}$} & \multicolumn{3}{|c|}{$N_{207} / N_{235}$} & \multicolumn{3}{|c|}{$N_{207} / N_{206}$} & \multicolumn{3}{|c|}{$N_{208} / N_{232}$} \\
\hline \multirow[b]{2}{*}{ Ratio } & \multicolumn{2}{|c|}{ Age } & \multirow[b]{2}{*}{ Ratio } & \multicolumn{2}{|c|}{ Age } & \multirow[b]{2}{*}{ Ratio } & \multicolumn{2}{|c|}{ Age } & \multirow[b]{2}{*}{ Ratio } & \multicolumn{2}{|c|}{ Age } \\
\hline & $\begin{array}{c}\begin{array}{c}\text { Number } \\
\text { of } \\
\text { years }\end{array} \\
\end{array}$ & $\begin{array}{c}\text { Error } \\
\pm\end{array}$ & & $\begin{array}{c}\text { Number } \\
\text { of } \\
\text { years }\end{array}$ & $\begin{array}{c}\text { Error } \\
\pm\end{array}$ & & \begin{tabular}{|c|}
$\begin{array}{c}\text { Number } \\
\text { of } \\
\text { years }\end{array}$ \\
\end{tabular} & $\begin{array}{c}\text { Error } \\
\pm\end{array}$ & & \begin{tabular}{|c|}
$\begin{array}{c}\text { Number } \\
\text { of } \\
\text { years }\end{array}$ \\
\end{tabular} & $\begin{array}{c}\text { Error } \\
\pm\end{array}$ \\
\hline $\begin{array}{l}0.031373 \\
0.031530 \\
0.031690 \\
0.031849 \\
0.032007\end{array}$ & $\begin{array}{l}201 \\
202 \\
203 \\
204 \\
205\end{array}$ & $\begin{array}{l}0.4 \\
0.4 \\
0.5 \\
0.5 \\
0.5\end{array}$ & $\begin{array}{l}0.215800 \\
0.216982 \\
0.218166 \\
0.219350 \\
0.220536\end{array}$ & $\begin{array}{l}201 \\
202 \\
203 \\
204 \\
205\end{array}$ & $\begin{array}{l}4.5 \\
4.5 \\
4.6 \\
4.6 \\
4.6\end{array}$ & $\begin{array}{l}0.049952 \\
0.049976 \\
0.049995 \\
0.050015 \\
0.050038\end{array}$ & $\begin{array}{l}201 \\
202 \\
203 \\
204 \\
205\end{array}$ & $\begin{array}{l}58.8 \\
59.0 \\
58.9 \\
58.8 \\
59.0\end{array}$ & $\begin{array}{l}0.009859 \\
0.009908 \\
0.009958 \\
0.010006 \\
0.010056\end{array}$ & $\begin{array}{l}201 \\
202 \\
203 \\
204 \\
205\end{array}$ & $\begin{array}{r}9.9 \\
10.0 \\
10.0 \\
10.1 \\
10.1\end{array}$ \\
\hline $\begin{array}{l}0.032166 \\
0.032325 \\
0.032482 \\
0.032641 \\
0.032800\end{array}$ & $\begin{array}{l}206 \\
207 \\
208 \\
209 \\
210\end{array}$ & $\begin{array}{l}0.5 \\
0.5 \\
0.5 \\
0.5 \\
0.5\end{array}$ & $\begin{array}{l}0.221722 \\
0.222913 \\
0.224101 \\
0.225291 \\
0.226484\end{array}$ & $\begin{array}{l}206 \\
207 \\
208 \\
209 \\
210\end{array}$ & $\begin{array}{l}4.6 \\
4.6 \\
4.7 \\
4.7 \\
4.7\end{array}$ & $\begin{array}{l}0.050058 \\
0.050079 \\
0.050103 \\
0.050124 \\
0.050145\end{array}$ & $\begin{array}{l}206 \\
207 \\
208 \\
209 \\
210\end{array}$ & $\begin{array}{l}58.9 \\
58.9 \\
59.1 \\
59.1 \\
59.0\end{array}$ & $\begin{array}{l}0.010105 \\
0.010155 \\
0.010204 \\
0.010253 \\
0.010302\end{array}$ & $\begin{array}{l}206 \\
207 \\
208 \\
209 \\
210\end{array}$ & $\begin{array}{l}10.2 \\
10.2 \\
10.3 \\
10.3 \\
10.4\end{array}$ \\
\hline $\begin{array}{l}0.032958 \\
0.033117 \\
0.033276 \\
0.033435 \\
0.033594\end{array}$ & $\begin{array}{l}211 \\
212 \\
213 \\
214 \\
215\end{array}$ & $\begin{array}{l}0.5 \\
0.5 \\
0.5 \\
0.5 \\
0.5\end{array}$ & $\begin{array}{l}0.227676 \\
0.228870 \\
0.230066 \\
0.231263 \\
0.232460\end{array}$ & $\begin{array}{l}211 \\
212 \\
213 \\
214 \\
215\end{array}$ & $\begin{array}{l}4.7 \\
4.8 \\
4.8 \\
4.8 \\
4.8\end{array}$ & $\begin{array}{l}0.050167 \\
0.050188 \\
0.050209 \\
0.050230 \\
0.050251\end{array}$ & $\begin{array}{l}211 \\
212 \\
213 \\
214 \\
215\end{array}$ & $\begin{array}{l}59.1 \\
59.1 \\
59.1 \\
59.1 \\
59.1\end{array}$ & $\begin{array}{l}0.010352 \\
0.010401 \\
0.010451 \\
0.010500 \\
0.010549\end{array}$ & $\begin{array}{l}211 \\
212 \\
213 \\
214 \\
215\end{array}$ & $\begin{array}{l}10.4 \\
10.5 \\
10.5 \\
10.5 \\
10.6\end{array}$ \\
\hline $\begin{array}{l}0.033754 \\
0.033913 \\
0.034071 \\
0.034230 \\
0.034389\end{array}$ & $\begin{array}{l}216 \\
217 \\
218 \\
219 \\
220\end{array}$ & $\begin{array}{l}0.5 \\
0.5 \\
0.5 \\
0.5 \\
0.5\end{array}$ & $\begin{array}{l}0.233660 \\
0.234858 \\
0.236060 \\
0.237262 \\
0.238466\end{array}$ & $\begin{array}{l}216 \\
217 \\
218 \\
219 \\
220\end{array}$ & $\begin{array}{l}4.8 \\
4.9 \\
4.9 \\
4.9 \\
4.9\end{array}$ & $\begin{array}{l}0.050271 \\
0.050292 \\
0.050315 \\
0.050337 \\
0.050358\end{array}$ & $\begin{array}{l}216 \\
217 \\
218 \\
219 \\
220\end{array}$ & $\begin{array}{l}59.0 \\
59.0 \\
59.2 \\
59.2 \\
59.2\end{array}$ & $\begin{array}{l}598 \\
648 \\
697 \\
747 \\
795\end{array}$ & $\begin{array}{l}216 \\
217 \\
218 \\
219 \\
220\end{array}$ & $\begin{array}{l}10.6 \\
10.7 \\
10.7 \\
10.8 \\
10.8\end{array}$ \\
\hline $\begin{array}{l}0.034547 \\
0.034707 \\
0.034866 \\
0.035024 \\
0.035183\end{array}$ & $\begin{array}{l}221 \\
222 \\
223 \\
224 \\
225\end{array}$ & $\begin{array}{l}0.5 \\
0.5 \\
0.5 \\
0.5 \\
0.5\end{array}$ & $\begin{array}{l}0.239669 \\
0.240875 \\
0.242083 \\
0.243291 \\
0.244500\end{array}$ & $\begin{array}{l}221 \\
222 \\
223 \\
224 \\
225\end{array}$ & $\begin{array}{l}5.0 \\
5.0 \\
5.0 \\
5.0 \\
5.0\end{array}$ & $\begin{array}{l}0.050381 \\
0.050401 \\
0.050422 \\
0.050445 \\
0.050467\end{array}$ & $\begin{array}{l}221 \\
222 \\
223 \\
224 \\
225\end{array}$ & $\begin{array}{l}59.3 \\
59.2 \\
59.2 \\
59.3 \\
59.3\end{array}$ & $\begin{array}{l}0.010845 \\
0.010894 \\
0.010944 \\
0.010993 \\
0.011043\end{array}$ & $\begin{array}{l}221 \\
222 \\
223 \\
224 \\
225\end{array}$ & $\begin{array}{l}10.9 \\
10.9 \\
11.0 \\
11.0 \\
111\end{array}$ \\
\hline $\begin{array}{l}0.035343 \\
0.035503 \\
0.035661 \\
0.035821 \\
0.035980\end{array}$ & $\begin{array}{l}226 \\
227 \\
228 \\
229 \\
230\end{array}$ & $\begin{array}{l}0.5 \\
0.5 \\
0.5 \\
0.5 \\
0.5\end{array}$ & $\begin{array}{l}0.245711 \\
0.246922 \\
0.248134 \\
0.249348 \\
0.250564\end{array}$ & $\begin{array}{l}226 \\
227 \\
228 \\
229 \\
230\end{array}$ & $\begin{array}{l}5.1 \\
5.1 \\
5.1 \\
5.1 \\
5.2\end{array}$ & $\begin{array}{l}0.050487 \\
0.050508 \\
0.050531 \\
0.050551 \\
0.050573\end{array}$ & $\begin{array}{l}226 \\
227 \\
228 \\
229 \\
230\end{array}$ & $\begin{array}{l}59.2 \\
59.3 \\
59.4 \\
59.3 \\
59.3\end{array}$ & $\begin{array}{l}0.011091 \\
0.011141 \\
0.011190 \\
0.011240 \\
0.011290\end{array}$ & $\begin{array}{l}226 \\
227 \\
228 \\
229 \\
230\end{array}$ & $\begin{array}{l}11.1 \\
11.2 \\
11.2 \\
11.3 \\
11.3\end{array}$ \\
\hline $\begin{array}{l}0.036139 \\
0.036298 \\
0.036458 \\
0.036616 \\
0.036776\end{array}$ & $\begin{array}{l}231 \\
232 \\
233 \\
234 \\
235\end{array}$ & $\begin{array}{l}0.5 \\
0.5 \\
0.5 \\
0.5 \\
0.5\end{array}$ & $\begin{array}{l}0.251779 \\
0.252998 \\
0.254216 \\
0.255436 \\
0.256658\end{array}$ & $\begin{array}{l}231 \\
232 \\
233 \\
234 \\
235\end{array}$ & $\begin{array}{l}5.2 \\
5.2 \\
5.2 \\
5.3 \\
5.3\end{array}$ & $\begin{array}{l}0.050595 \\
0.050617 \\
0.050637 \\
0.050661 \\
0.050682\end{array}$ & $\begin{array}{l}231 \\
232 \\
233 \\
234 \\
235\end{array}$ & $\begin{array}{l}59.4 \\
59.4 \\
59.4 \\
59.5 \\
59.5\end{array}$ & $\begin{array}{l}0.011338 \\
0.011388 \\
0.011437 \\
0.011487 \\
0.011536\end{array}$ & $\begin{array}{l}231 \\
232 \\
233 \\
234 \\
235\end{array}$ & $\begin{array}{l}11.4 \\
11.4 \\
11.5 \\
11.5 \\
11.6\end{array}$ \\
\hline $\begin{array}{l}0.036935 \\
0.037095 \\
0.037254 \\
0.037414 \\
0.037574\end{array}$ & $\begin{array}{l}236 \\
237 \\
238 \\
239 \\
240\end{array}$ & $\begin{array}{l}0.5 \\
0.5 \\
0.5 \\
0.5 \\
0.5\end{array}$ & $\begin{array}{l}0.257879 \\
0.259103 \\
0.260328 \\
0.261554 \\
0.262780\end{array}$ & $\begin{array}{l}236 \\
237 \\
238 \\
239 \\
240\end{array}$ & $\begin{array}{l}5.3 \\
5.3 \\
5.3 \\
5.4 \\
5.4\end{array}$ & $\begin{array}{l}0.050704 \\
0.050725 \\
0.050747 \\
0.050768 \\
0.050789\end{array}$ & $\begin{array}{l}236 \\
237 \\
238 \\
239 \\
240\end{array}$ & $\begin{array}{l}59.5 \\
59.5 \\
59.5 \\
59.5 \\
59.5\end{array}$ & $\begin{array}{l}0.011585 \\
0.011634 \\
0.011684 \\
0.011734 \\
0.011783\end{array}$ & $\begin{array}{l}236 \\
237 \\
238 \\
239 \\
240\end{array}$ & $\begin{array}{l}11.6 \\
11.7 \\
11.7 \\
118 \\
11.8\end{array}$ \\
\hline $\begin{array}{l}0.037732 \\
0.037892 \\
0.038052 \\
0.038211 \\
0.038370\end{array}$ & $\begin{array}{l}241 \\
242 \\
243 \\
244 \\
245\end{array}$ & $\begin{array}{l}0.5 \\
0.5 \\
0.5 \\
0.5 \\
0.5\end{array}$ & $\begin{array}{l}0.264008 \\
0.265238 \\
0.266469 \\
0.267701 \\
0.268934\end{array}$ & $\begin{array}{l}241 \\
242 \\
243 \\
244 \\
245\end{array}$ & $\begin{array}{l}5.4 \\
5.4 \\
5.5 \\
5.5 \\
5.5\end{array}$ & $\begin{array}{l}0.050812 \\
0.050833 \\
0.050855 \\
0.050877 \\
0.050900\end{array}$ & $\begin{array}{l}241 \\
242 \\
243 \\
244 \\
245\end{array}$ & $\begin{array}{l}59.6 \\
59.5 \\
59.6 \\
59.6 \\
59.7\end{array}$ & $\begin{array}{l}0.011833 \\
0.011881 \\
0.011931 \\
0.011980 \\
0.012030\end{array}$ & $\begin{array}{l}241 \\
242 \\
243 \\
244 \\
245\end{array}$ & $\begin{array}{l}11.9 \\
11.9 \\
12.0 \\
12.0 \\
12.1\end{array}$ \\
\hline $\begin{array}{l}0.038530 \\
0.038690 \\
0.038850 \\
0.039010 \\
0.039169\end{array}$ & $\begin{array}{l}246 \\
247 \\
248 \\
249 \\
250\end{array}$ & $\begin{array}{l}0.5 \\
0.5 \\
0.5 \\
0.6 \\
0.6\end{array}$ & $\begin{array}{l}0.270168 \\
0.271403 \\
0.272639 \\
0.273878 \\
0.2751_{16}\end{array}$ & $\begin{array}{l}246 \\
247 \\
248 \\
249 \\
250\end{array}$ & $\begin{array}{l}5.5 \\
5.5 \\
5.6 \\
5.6 \\
5.6\end{array}$ & $\begin{array}{l}0.050921 \\
0.050942 \\
0.050963 \\
0.050985 \\
0.051008\end{array}$ & $\begin{array}{l}246 \\
247 \\
248 \\
249 \\
250\end{array}$ & $\begin{array}{l}59.7 \\
59.6 \\
59.6 \\
59.6\end{array}$ & $\begin{array}{l}0.012080 \\
0.012128 \\
0.012178 \\
0.012227 \\
0.012277\end{array}$ & $\begin{array}{l}246 \\
247 \\
248 \\
249 \\
250\end{array}$ & $\begin{array}{l}12.1 \\
12.2 \\
12.2 \\
123 \\
12.3\end{array}$ \\
\hline
\end{tabular}


Geologic age, in millions of years, calculated from the atomic ratios of indicated isotopes

\begin{tabular}{|c|c|c|c|c|c|c|c|c|c|c|c|}
\hline \multicolumn{3}{|c|}{$N_{206} / N_{238}$} & \multicolumn{3}{|c|}{$N_{207} / N_{235}$} & \multicolumn{3}{|c|}{$N_{207} / N_{206}$} & \multicolumn{3}{|c|}{$N_{208} / N_{232}$} \\
\hline \multirow[b]{2}{*}{ Ratio } & \multicolumn{2}{|c|}{ Age } & \multirow[b]{2}{*}{ Ratio } & \multicolumn{2}{|c|}{ Age } & \multirow[b]{2}{*}{ Ratio } & \multicolumn{2}{|c|}{ Age } & \multirow[b]{2}{*}{ Ratio } & \multicolumn{2}{|c|}{ Age } \\
\hline & \begin{tabular}{|c|}
$\begin{array}{c}\text { Number } \\
\text { of } \\
\text { years }\end{array}$ \\
\end{tabular} & $\begin{array}{c}\text { Error } \\
\pm\end{array}$ & & \begin{tabular}{|c|} 
Number \\
of \\
years
\end{tabular} & $\begin{array}{c}\text { Error } \\
\pm\end{array}$ & & \begin{tabular}{|c|}
$\begin{array}{c}\text { Number } \\
\text { of } \\
\text { years }\end{array}$ \\
\end{tabular} & $\begin{array}{c}\text { Error } \\
\pm\end{array}$ & & \begin{tabular}{|c|}
$\begin{array}{c}\text { Numl or } \\
\text { of } \\
\text { years }\end{array}$ \\
\end{tabular} & $\begin{array}{c}\text { Error } \\
\pm\end{array}$ \\
\hline $\begin{array}{l}0.039329 \\
0.039489 \\
0.039648 \\
0.039807 \\
0.039968\end{array}$ & $\begin{array}{l}251 \\
252 \\
253 \\
254 \\
255\end{array}$ & $\begin{array}{l}0.6 \\
0.6 \\
0.6 \\
0.6 \\
0.6\end{array}$ & $\begin{array}{l}0.276357 \\
0.277599 \\
0.278841 \\
0.280084 \\
0.281330\end{array}$ & $\begin{array}{l}251 \\
252 \\
253 \\
254 \\
255\end{array}$ & $\begin{array}{l}5.6 \\
5.7 \\
5.7 \\
5.7 \\
5.7\end{array}$ & $\begin{array}{l}0.051029 \\
0.051051 \\
0.051074 \\
0.051096 \\
0.051117\end{array}$ & $\begin{array}{l}251 \\
252 \\
253 \\
254 \\
255\end{array}$ & $\begin{array}{l}59.7 \\
59.7 \\
59.8 \\
59.8 \\
59.7\end{array}$ & $\begin{array}{l}0.012327 \\
0.012375 \\
0.012425 \\
0.012474 \\
0.012524\end{array}$ & $\begin{array}{l}251 \\
252 \\
253 \\
254 \\
255\end{array}$ & $\begin{array}{l}12.4 \\
12.4 \\
12.5 \\
12.5 \\
12.6\end{array}$ \\
\hline $\begin{array}{l}0.040129 \\
0.040288 \\
0.040448 \\
0.040608 \\
0.040767\end{array}$ & $\begin{array}{l}256 \\
257 \\
258 \\
259 \\
260\end{array}$ & $\begin{array}{l}0.6 \\
0.6 \\
0.6 \\
0.6 \\
0.6\end{array}$ & $\begin{array}{l}0.282575 \\
0.283825 \\
0.285072 \\
0.286322 \\
0.287574\end{array}$ & $\begin{array}{l}256 \\
257 \\
258 \\
259 \\
260\end{array}$ & $\begin{array}{l}5.7 \\
5.8 \\
5.8 \\
5.8 \\
5.8\end{array}$ & $\begin{array}{l}0.051137 \\
0.051161 \\
0.051182 \\
0.051204 \\
0.051227\end{array}$ & $\begin{array}{l}256 \\
257 \\
258 \\
259 \\
260\end{array}$ & $\begin{array}{l}59.7 \\
59.8 \\
59.8 \\
59.8 \\
59.8\end{array}$ & $\begin{array}{l}0.012574 \\
0.012623 \\
0.012672 \\
0.012721 \\
0.012771\end{array}$ & $\begin{array}{l}256 \\
257 \\
258 \\
259 \\
260\end{array}$ & $\begin{array}{l}12.6 \\
12.7 \\
12.7 \\
12.8 \\
12.8\end{array}$ \\
\hline $\begin{array}{l}0,040927 \\
0,041087 \\
0.041246 \\
0.041408 \\
0.041568\end{array}$ & $\begin{array}{l}261 \\
262 \\
263 \\
264 \\
265\end{array}$ & $\begin{array}{l}0.6 \\
0.6 \\
0.6 \\
0.6 \\
0.6\end{array}$ & $\begin{array}{l}0.288825 \\
0.290078 \\
0.291335 \\
0.292590 \\
0.293847\end{array}$ & $\begin{array}{l}261 \\
262 \\
263 \\
264 \\
265\end{array}$ & $\begin{array}{l}5.9 \\
5.9 \\
5.9 \\
59\end{array}$ & $\begin{array}{l}0.051249 \\
0.051271 \\
0.051295 \\
0.051314 \\
0.051336\end{array}$ & $\begin{array}{l}261 \\
262 \\
263 \\
264 \\
265\end{array}$ & $\begin{array}{l}59.9 \\
59.9 \\
60.0 \\
59.9 \\
59.9\end{array}$ & $\begin{array}{l}0.012821 \\
0.012870 \\
0.012919 \\
0.012969 \\
0.013018\end{array}$ & $\begin{array}{l}261 \\
262 \\
263 \\
264 \\
265\end{array}$ & $\begin{array}{l}12.9 \\
12.9 \\
13.0 \\
13.0 \\
13.1\end{array}$ \\
\hline $\begin{array}{l}0.041728 \\
0.041887 \\
0.042048 \\
0.042208 \\
0.042367\end{array}$ & $\begin{array}{l}266 \\
267 \\
268 \\
269 \\
270\end{array}$ & $\begin{array}{l}0.6 \\
0.6 \\
0.6 \\
0.6 \\
0.6\end{array}$ & $\begin{array}{l}0.295106 \\
0.296365 \\
0.297625 \\
0.298889 \\
0.300152\end{array}$ & $\begin{array}{l}266 \\
267 \\
268 \\
269 \\
270\end{array}$ & $\begin{array}{l}6.0 \\
6.0\end{array}$ & $\begin{array}{l}0.051358 \\
0.051382 \\
0.051403 \\
0.051425 \\
0.051449\end{array}$ & $\begin{array}{l}266 \\
267 \\
268 \\
269 \\
270\end{array}$ & $\begin{array}{l}59.9 \\
60.0 \\
60.0 \\
60.0 \\
60.1\end{array}$ & $\begin{array}{l}3068 \\
3117 \\
3166 \\
3216 \\
3265\end{array}$ & $\begin{array}{l}266 \\
267 \\
268 \\
269 \\
270\end{array}$ & $\begin{array}{l}13.1 \\
13.2 \\
13.2 \\
13.3 \\
13.3\end{array}$ \\
\hline $\begin{array}{l}0.042529 \\
0.042689 \\
0.042849 \\
0.043009 \\
0.043170\end{array}$ & $\begin{array}{l}271 \\
272 \\
273 \\
274 \\
275\end{array}$ & $\begin{array}{l}0.6 \\
0.6 \\
0.6 \\
0.6 \\
0.6\end{array}$ & $\begin{array}{l}0.301416 \\
0.302681 \\
0.303948 \\
0.305216 \\
0.306486\end{array}$ & $\begin{array}{l}271 \\
272 \\
273 \\
274 \\
275\end{array}$ & $\begin{array}{l}6.1 \\
6.1 \\
6.1\end{array}$ & $\begin{array}{l}0.051469 \\
0.051491 \\
0.051513 \\
0.051536 \\
0.051557\end{array}$ & $\begin{array}{l}271 \\
272 \\
273 \\
274 \\
275\end{array}$ & $\begin{array}{l}60.0 \\
60.0 \\
60.0 \\
60.1 \\
60.0\end{array}$ & $\begin{array}{l}3315 \\
3365 \\
3414 \\
3463 \\
3513\end{array}$ & $\begin{array}{l}271 \\
272 \\
273 \\
274 \\
275\end{array}$ & $\begin{array}{l}13.4 \\
13.4 \\
13.5 \\
13.5 \\
13.6\end{array}$ \\
\hline $\begin{array}{l}0.043329 \\
0.043490 \\
0.043650 \\
0.043812 \\
0.043971\end{array}$ & $\begin{array}{l}276 \\
277 \\
278 \\
279 \\
280\end{array}$ & $\begin{array}{l}0.6 \\
0.6 \\
0.6 \\
0.6 \\
0.6\end{array}$ & $\begin{array}{l}0.307757 \\
0.309030 \\
0.310302 \\
0.311577 \\
0.312852\end{array}$ & $\begin{array}{l}276 \\
277 \\
278 \\
279 \\
280\end{array}$ & $\begin{array}{l}6.2 \\
6.2 \\
6.2 \\
6.3\end{array}$ & $\begin{array}{l}0.051581 \\
0.051603 \\
0.051625 \\
0.051646 \\
0.051670\end{array}$ & $\begin{array}{l}276 \\
277 \\
278 \\
279 \\
280\end{array}$ & $\begin{array}{l}60.1 \\
60.2 \\
60.2 \\
60.1 \\
60.2\end{array}$ & $\begin{array}{l}3562 \\
3612 \\
3662 \\
3710 \\
3760\end{array}$ & $\begin{array}{l}276 \\
277 \\
278 \\
279 \\
280\end{array}$ & $\begin{array}{l}13.6 \\
13.7 \\
13.7 \\
13.8 \\
13.8\end{array}$ \\
\hline $\begin{array}{l}0.044132 \\
0.044293 \\
0.044452 \\
0.044613 \\
0.044775\end{array}$ & $\begin{array}{l}281 \\
282 \\
283 \\
284 \\
285\end{array}$ & $\begin{array}{l}0.6 \\
0.6 \\
0.6 \\
0.6 \\
0.6\end{array}$ & $\begin{array}{l}0.314128 \\
0.315407 \\
0.316687 \\
0.317968 \\
0.319249\end{array}$ & $\begin{array}{l}281 \\
282 \\
283 \\
284 \\
285\end{array}$ & $\begin{array}{l}6.3 \\
6.3 \\
6.4 \\
6.4 \\
6.4\end{array}$ & $\begin{array}{l}0.051691 \\
0.051713 \\
0.051737 \\
0.051759 \\
0.051779\end{array}$ & $\begin{array}{l}281 \\
282 \\
283 \\
284 \\
285\end{array}$ & $\begin{array}{l}60.1 \\
60.2 \\
60.3 \\
60.3 \\
60.2\end{array}$ & $\begin{array}{l}0.013810 \\
0.013859 \\
0.013909 \\
0.013958 \\
0.014007\end{array}$ & $\begin{array}{l}2 \& 1 \\
2 \& ? \\
2 \varepsilon 3 \\
2 \& 4 \\
2 \& 5\end{array}$ & $\begin{array}{l}13.9 \\
13.9 \\
14.0 \\
14.0 \\
14.0\end{array}$ \\
\hline $\begin{array}{l}0.044935 \\
0.045095 \\
0.045256 \\
0.045416 \\
0.045577\end{array}$ & $\begin{array}{l}286 \\
287 \\
288 \\
289 \\
290\end{array}$ & $\begin{array}{l}0.6 \\
0.6 \\
0.6 \\
0.6 \\
0.6\end{array}$ & $\begin{array}{l}0.320532 \\
0.321817 \\
0.323102 \\
0.324390 \\
0.325678\end{array}$ & $\begin{array}{l}286 \\
287 \\
288 \\
289 \\
290\end{array}$ & $\begin{array}{l}6.4 \\
6.4 \\
6.5 \\
6.5 \\
6.5\end{array}$ & $\begin{array}{l}0.051802 \\
0.051825 \\
0.051847 \\
0.051871 \\
0.051892\end{array}$ & $\begin{array}{l}286 \\
287 \\
288 \\
289 \\
290\end{array}$ & $\begin{array}{l}60.2 \\
60.3 \\
60.3 \\
60.4 \\
60.3\end{array}$ & $\begin{array}{l}0.014057 \\
0.014107 \\
0.014156 \\
0.014206 \\
0.014255\end{array}$ & $\begin{array}{l}2 \& 6 \\
2 \varepsilon 7 \\
2 \varepsilon 8 \\
2 \varepsilon ? \\
2 \subsetneq 7\end{array}$ & $\begin{array}{l}14.1 \\
14.1 \\
14.2 \\
14.2 \\
14.3\end{array}$ \\
\hline $\begin{array}{l}0.045738 \\
0.045900 \\
0.046059 \\
0.046220 \\
0.046381\end{array}$ & $\begin{array}{l}291 \\
292 \\
293 \\
294 \\
295\end{array}$ & $\begin{array}{l}0.6 \\
0.6 \\
0.6 \\
0.7 \\
0.7\end{array}$ & $\begin{array}{l}0.326967 \\
0.328258 \\
0.329549 \\
0.330844 \\
0.332138\end{array}$ & $\begin{array}{l}291 \\
292 \\
293 \\
294 \\
295\end{array}$ & $\begin{array}{l}6.5 \\
6.6 \\
6.6 \\
6.6 \\
6.6\end{array}$ & $\begin{array}{l}0.051914 \\
0.051936 \\
0.051960 \\
0.051982 \\
0.052004\end{array}$ & $\begin{array}{l}291 \\
292 \\
293 \\
294 \\
295\end{array}$ & $\begin{array}{l}60.3 \\
60.3 \\
60.4 \\
60.4 \\
60.4\end{array}$ & $\begin{array}{l}0.014304 \\
0.014354 \\
0.014404 \\
0.014453 \\
0.014502\end{array}$ & $\begin{array}{l}2 \subsetneq 1 \\
2 \subseteq 2 \\
2 \subsetneq 3 \\
2 \subsetneq 4 \\
2 \div 5\end{array}$ & $\begin{array}{l}14.3 \\
14.4 \\
14.4 \\
14.5 \\
14.5\end{array}$ \\
\hline $\begin{array}{l}0.046541 \\
0.046702 \\
0.046863 \\
0.047024 \\
0.047185\end{array}$ & $\begin{array}{l}296 \\
297 \\
298 \\
299 \\
300\end{array}$ & $\begin{array}{l}0.7 \\
0.7 \\
0.7 \\
0.7 \\
0.7\end{array}$ & $\begin{array}{l}0.333433 \\
0.334729 \\
0.336027 \\
0.337327 \\
0.338627\end{array}$ & $\begin{array}{l}296 \\
297 \\
298 \\
299 \\
300\end{array}$ & $\begin{array}{l}6.6 \\
6.7 \\
6.7 \\
6.7 \\
6.7\end{array}$ & $\begin{array}{l}0.052028 \\
0.052050 \\
0.052072 \\
0.052095 \\
0.052117\end{array}$ & $\begin{array}{l}296 \\
297 \\
298 \\
299 \\
300\end{array}$ & $\begin{array}{l}60.5 \\
60.5 \\
60.5 \\
60.5 \\
60.5\end{array}$ & $\begin{array}{l}0.014552 \\
0.014602 \\
0.014651 \\
0.014701 \\
0.014751\end{array}$ & $\begin{array}{l}255 \\
2 c 7 \\
2 c 8 \\
2 c 9 \\
3 c 0\end{array}$ & $\begin{array}{l}14.6 \\
14.6 \\
14.7 \\
14.7 \\
14.8\end{array}$ \\
\hline
\end{tabular}


$301-350$

Geologic age, in millions of years, calculated from the atomic ratios of indicated isotopes

\begin{tabular}{|c|c|c|c|c|c|c|c|c|c|c|c|}
\hline \multicolumn{3}{|c|}{$N_{206} / N_{238}$} & \multicolumn{3}{|c|}{$N_{207} / N_{235}$} & \multicolumn{3}{|c|}{$N_{207} / N_{206}$} & \multicolumn{3}{|c|}{$N_{208} / N_{232}$} \\
\hline \multirow[b]{2}{*}{ Ratio } & \multicolumn{2}{|c|}{ Age } & \multirow[b]{2}{*}{ Ratio } & \multicolumn{2}{|c|}{ Age } & \multirow[b]{2}{*}{ Ratio } & \multicolumn{2}{|c|}{ Age } & \multirow[b]{2}{*}{ Ratio } & \multicolumn{2}{|c|}{ Aq $\mathrm{e}$} \\
\hline & \begin{tabular}{|c|}
$\begin{array}{c}\text { Number } \\
\text { of } \\
\text { years }\end{array}$ \\
\end{tabular} & $\begin{array}{c}\text { Error } \\
\pm\end{array}$ & & \begin{tabular}{|c|}
$\begin{array}{c}\text { Number } \\
\text { of } \\
\text { years }\end{array}$ \\
\end{tabular} & $\begin{array}{c}\text { Error } \\
\pm\end{array}$ & & \begin{tabular}{|c|} 
Number \\
of \\
years
\end{tabular} & $\begin{array}{c}\text { Error } \\
\pm\end{array}$ & & \begin{tabular}{|c|}
$\begin{array}{c}\text { Number } \\
\text { of } \\
\text { years }\end{array}$ \\
\end{tabular} & $\begin{array}{c}\text { Error } \\
\pm\end{array}$ \\
\hline $\begin{array}{l}0.047347 \\
0.047507 \\
0.047668 \\
0.047829 \\
0.047990\end{array}$ & $\begin{array}{l}301 \\
302 \\
303 \\
304 \\
305\end{array}$ & $\begin{array}{l}0.7 \\
0.7 \\
0.7 \\
0.7 \\
0.7\end{array}$ & $\begin{array}{l}0.339930 \\
0.341234 \\
0.342537 \\
0.343843 \\
0.345151\end{array}$ & $\begin{array}{l}301 \\
302 \\
303 \\
304 \\
305\end{array}$ & $\begin{array}{l}6.8 \\
6.8 \\
6.8 \\
6.8 \\
6.8\end{array}$ & $\begin{array}{l}0.052139 \\
0.052162 \\
0.052185 \\
0.052207 \\
0.052230\end{array}$ & $\begin{array}{l}301 \\
302 \\
303 \\
304 \\
305\end{array}$ & $\begin{array}{l}60.5 \\
60.5 \\
60.6 \\
60.6 \\
60.6\end{array}$ & $\begin{array}{l}0.014799 \\
0.014849 \\
0.014899 \\
0.014949 \\
0.014998\end{array}$ & $\begin{array}{l}301 \\
302 \\
303 \\
304 \\
305\end{array}$ & $\begin{array}{l}14.8 \\
14.9 \\
14.9 \\
15.0 \\
15.0\end{array}$ \\
\hline $\begin{array}{l}0.048151 \\
0.048313 \\
0.048474 \\
0.048634 \\
0.048796\end{array}$ & $\begin{array}{l}306 \\
307 \\
308 \\
309 \\
310\end{array}$ & $\begin{array}{l}0.7 \\
0.7 \\
0.7 \\
0.7 \\
0.7\end{array}$ & $\begin{array}{l}0.346459 \\
0.347769 \\
0.349080 \\
0.350393 \\
0.351705\end{array}$ & $\begin{array}{l}306 \\
307 \\
308 \\
309 \\
310\end{array}$ & $\begin{array}{l}6.9 \\
6.9 \\
6.9 \\
6.9 \\
7.0\end{array}$ & $\begin{array}{l}0.052253 \\
0.052274 \\
0.052297 \\
0.052321 \\
0.052343\end{array}$ & $\begin{array}{l}306 \\
307 \\
308 \\
309 \\
310\end{array}$ & $\begin{array}{l}60.6 \\
60.6 \\
60.6 \\
60.7 \\
60.7\end{array}$ & $\begin{array}{l}0.015047 \\
0.015097 \\
0.015147 \\
0.015196 \\
0.015246\end{array}$ & $\begin{array}{l}306 \\
307 \\
308 \\
309 \\
310\end{array}$ & $\begin{array}{l}15.1 \\
15.1 \\
15.2 \\
15.2 \\
15.3\end{array}$ \\
\hline $\begin{array}{l}0.048958 \\
0.049118 \\
0.049280 \\
0.049441 \\
0.049601\end{array}$ & $\begin{array}{l}311 \\
312 \\
313 \\
314 \\
315\end{array}$ & $\begin{array}{l}0.7 \\
0.7 \\
0.7 \\
0.7 \\
0.7\end{array}$ & $\begin{array}{l}0.353022 \\
0.354336 \\
0.355654 \\
0.356973 \\
0.358293\end{array}$ & $\begin{array}{l}311 \\
312 \\
313 \\
314 \\
315\end{array}$ & $\begin{array}{l}7.0 \\
7.0 \\
7.0 \\
7.0 \\
7.1\end{array}$ & $\begin{array}{l}0.052365 \\
0.052389 \\
0.052411 \\
0.052434 \\
0.052458\end{array}$ & $\begin{array}{l}311 \\
312 \\
313 \\
314 \\
315\end{array}$ & $\begin{array}{l}60.7 \\
60.7 \\
60.7 \\
60.8 \\
60.8\end{array}$ & $\begin{array}{l}0.015295 \\
0.015344 \\
0.015394 \\
0.015444 \\
0.015494\end{array}$ & $\begin{array}{l}311 \\
312 \\
313 \\
314 \\
315\end{array}$ & $\begin{array}{l}15.3 \\
15.4 \\
15.4 \\
15.5 \\
15.5\end{array}$ \\
\hline $\begin{array}{l}0.049764 \\
0.049925 \\
0.050086 \\
0.050247 \\
0.050409\end{array}$ & $\begin{array}{l}316 \\
317 \\
318 \\
319 \\
320\end{array}$ & $\begin{array}{l}0.7 \\
0.7 \\
0.7 \\
0.7 \\
0.7\end{array}$ & $\begin{array}{l}0.359613 \\
0.360935 \\
0.362259 \\
0.363584 \\
0.364911\end{array}$ & $\begin{array}{l}316 \\
317 \\
318 \\
319 \\
320\end{array}$ & $\begin{array}{l}7.1 \\
7.1 \\
7.1 \\
7.2 \\
7.2\end{array}$ & $\begin{array}{l}479 \\
502 \\
525 \\
548 \\
570\end{array}$ & $\begin{array}{l}316 \\
317 \\
318 \\
319 \\
320\end{array}$ & $\begin{array}{l}60.8 \\
60.8 \\
60.8 \\
60.8 \\
60.8\end{array}$ & $\begin{array}{l}0.015543 \\
0.015592 \\
0.015642 \\
0.015692 \\
0.015741\end{array}$ & $\begin{array}{l}316 \\
317 \\
318 \\
319 \\
320\end{array}$ & $\begin{array}{l}15.6 \\
15.6 \\
15.7 \\
15.7 \\
15.8\end{array}$ \\
\hline $\begin{array}{l}0.050570 \\
0.050732 \\
0.050894 \\
0.051055 \\
0.051216\end{array}$ & $\begin{array}{l}321 \\
322 \\
323 \\
324 \\
325\end{array}$ & $\begin{array}{l}0.7 \\
0.7 \\
0.7 \\
0.7 \\
0.7\end{array}$ & $\begin{array}{l}0.366238 \\
0.367568 \\
0.368898 \\
0.370228 \\
0.371561\end{array}$ & $\begin{array}{l}321 \\
322 \\
323 \\
324 \\
325\end{array}$ & $\begin{array}{l}7.2 \\
7.2 \\
7.2 \\
7.3 \\
7.3\end{array}$ & $\begin{array}{l}2594 \\
2616 \\
2638 \\
2661 \\
2685\end{array}$ & $\begin{array}{l}321 \\
322 \\
323 \\
324 \\
325\end{array}$ & $\begin{array}{l}60.9 \\
60.9 \\
60.9 \\
60.9 \\
61.0\end{array}$ & $\begin{array}{l}0.015791 \\
0.015840 \\
0.015890 \\
0.015940 \\
0.015989\end{array}$ & $\begin{array}{l}321 \\
322 \\
323 \\
324 \\
325\end{array}$ & $\begin{array}{l}15.8 \\
15.9 \\
15.9 \\
16.0 \\
16.0\end{array}$ \\
\hline $\begin{array}{l}0.051378 \\
0.051540 \\
0.051701 \\
0.051863 \\
0.052025\end{array}$ & $\begin{array}{l}326 \\
327 \\
328 \\
329 \\
330\end{array}$ & $\begin{array}{l}0.7 \\
0.7 \\
0.7 \\
0.7 \\
0.7\end{array}$ & $\begin{array}{l}0.372896 \\
0.374231 \\
0.375567 \\
0.376905 \\
0.378244\end{array}$ & $\begin{array}{l}326 \\
327 \\
328 \\
329 \\
330\end{array}$ & $\begin{array}{l}7.3 \\
7.3 \\
7.4 \\
7.4 \\
7.4\end{array}$ & $\begin{array}{l}2708 \\
2730 \\
2753 \\
2776 \\
2799\end{array}$ & $\begin{array}{l}326 \\
327 \\
328 \\
329 \\
330\end{array}$ & $\begin{array}{l}61.0 \\
61.0 \\
61.0 \\
61.0 \\
61.0\end{array}$ & $\begin{array}{l}0.016039 \\
0.016088 \\
0.016138 \\
0.016187 \\
0.016237\end{array}$ & $\begin{array}{l}326 \\
327 \\
328 \\
329 \\
330\end{array}$ & $\begin{array}{l}16.1 \\
16.1 \\
16.2 \\
16.2 \\
16.3\end{array}$ \\
\hline $\begin{array}{l}0.052185 \\
0.052348 \\
0.052510 \\
0.052672 \\
0.052833\end{array}$ & $\begin{array}{l}331 \\
332 \\
333 \\
334 \\
335\end{array}$ & $\begin{array}{l}0.7 \\
0.7 \\
0.7 \\
0.7 \\
0.7\end{array}$ & $\begin{array}{l}0.379584 \\
0.380928 \\
0.382270 \\
0.383615 \\
0.384959\end{array}$ & $\begin{array}{l}331 \\
332 \\
333 \\
334 \\
335\end{array}$ & $\begin{array}{l}7.4 \\
7.5 \\
7.5 \\
7.5 \\
7.5\end{array}$ & $\begin{array}{l}0.052823 \\
0.052845 \\
0.052868 \\
0.052891 \\
0.052914\end{array}$ & $\begin{array}{l}331 \\
332 \\
333 \\
334 \\
335\end{array}$ & $\begin{array}{l}61.1 \\
61.1 \\
61.1 \\
61.1 \\
61.1\end{array}$ & $\begin{array}{l}0.016287 \\
0.016337 \\
0.016386 \\
0.016435 \\
0.016485\end{array}$ & $\begin{array}{l}331 \\
332 \\
333 \\
334 \\
335\end{array}$ & $\begin{array}{l}16.3 \\
16.4 \\
16.4 \\
16.5 \\
16.5\end{array}$ \\
\hline $\begin{array}{l}0.052995 \\
0.053158 \\
0.053319 \\
0.053481 \\
0.053643\end{array}$ & $\begin{array}{l}336 \\
337 \\
338 \\
339 \\
340\end{array}$ & $\begin{array}{l}0.7 \\
0.7 \\
0.7 \\
0.8 \\
0.8\end{array}$ & $\begin{array}{l}0.386306 \\
0.387654 \\
0.389004 \\
0.390356 \\
0.391708\end{array}$ & $\begin{array}{l}336 \\
337 \\
338 \\
339 \\
340\end{array}$ & $\begin{array}{l}7.5 \\
7.6 \\
7.6 \\
7.6 \\
7.6\end{array}$ & $\begin{array}{l}0.052937 \\
0.052959 \\
0.052983 \\
0.053006 \\
0.053029\end{array}$ & $\begin{array}{l}336 \\
337 \\
338 \\
339 \\
340\end{array}$ & $\begin{array}{l}61.1 \\
61.1 \\
61.2 \\
61.2 \\
61.2\end{array}$ & $\begin{array}{l}0.016535 \\
0.016585 \\
0.016634 \\
0.016683 \\
0.016733\end{array}$ & $\begin{array}{l}336 \\
337 \\
338 \\
339 \\
340\end{array}$ & $\begin{array}{l}16.6 \\
16.6 \\
16.7 \\
16.7 \\
16.8\end{array}$ \\
\hline $\begin{array}{l}0.053804 \\
0.053967 \\
0.054129 \\
0.054290 \\
0.054452\end{array}$ & $\begin{array}{l}341 \\
342 \\
343 \\
344 \\
345\end{array}$ & $\begin{array}{l}0.8 \\
0.8 \\
0.8 \\
0.8 \\
0.8\end{array}$ & $\begin{array}{l}0.393062 \\
0.394416 \\
0.395773 \\
0.397131 \\
0.398489\end{array}$ & $\begin{array}{l}341 \\
342 \\
343 \\
344 \\
345\end{array}$ & $\begin{array}{l}7.7 \\
7.7 \\
7.7 \\
7.7 \\
7.7\end{array}$ & $\begin{array}{l}0.053053 \\
0.053075 \\
0.053098 \\
0.053122 \\
0.053145\end{array}$ & $\begin{array}{l}341 \\
342 \\
343 \\
344 \\
345\end{array}$ & $\begin{array}{l}61.2 \\
61.2 \\
61.2 \\
61.2 \\
61.3\end{array}$ & $\begin{array}{l}0.016783 \\
0.016833 \\
0.016882 \\
0.016931 \\
0.016981\end{array}$ & $\begin{array}{l}341 \\
342 \\
343 \\
344 \\
345\end{array}$ & $\begin{array}{l}16.8 \\
16.9 \\
16.9 \\
17.0 \\
17.0\end{array}$ \\
\hline $\begin{array}{l}0.054615 \\
0.054778 \\
0.054939 \\
0.055101 \\
0.055263\end{array}$ & $\begin{array}{l}346 \\
347 \\
348 \\
349 \\
350\end{array}$ & $\begin{array}{l}0.8 \\
0.8 \\
0.8 \\
0.8 \\
0.8\end{array}$ & $\begin{array}{l}0.399849 \\
0.401211 \\
0.402574 \\
0.403939 \\
0.405303\end{array}$ & $\begin{array}{l}346 \\
347 \\
348 \\
349 \\
350\end{array}$ & $\begin{array}{l}7.8 \\
7.8 \\
7.8 \\
7.8 \\
7.9\end{array}$ & $\begin{array}{l}0,053167 \\
0.053190 \\
0.053214 \\
0.053238 \\
0.053261\end{array}$ & $\begin{array}{l}346 \\
347 \\
348 \\
349 \\
350\end{array}$ & $\begin{array}{l}61.3 \\
61.2 \\
61.3 \\
61.3 \\
61.3\end{array}$ & $\begin{array}{l}0.017031 \\
0.017081 \\
0.017131 \\
0.017180 \\
0.017229\end{array}$ & $\begin{array}{l}346 \\
347 \\
348 \\
349 \\
350\end{array}$ & $\begin{array}{l}17.1 \\
17.1 \\
17.2 \\
17.2 \\
17.3\end{array}$ \\
\hline
\end{tabular}


Geologic age, in millions of years, calculated from the atomic ratios of indicated isotopes

\begin{tabular}{|c|c|c|c|c|c|c|c|c|c|c|c|}
\hline \multicolumn{3}{|c|}{$N_{206} / N_{238}$} & \multicolumn{3}{|c|}{$N_{207} / N_{235}$} & \multicolumn{3}{|c|}{$N_{207} / N_{206}$} & \multicolumn{3}{|c|}{$N_{208} / N_{232}$} \\
\hline \multirow[b]{2}{*}{ Ratio } & \multicolumn{2}{|c|}{ Age } & \multirow[b]{2}{*}{ Ratio } & \multicolumn{2}{|c|}{ Age } & \multirow[b]{2}{*}{ Ratio } & \multicolumn{2}{|c|}{ Age } & \multirow[b]{2}{*}{ Ratio } & \multicolumn{2}{|c|}{ Age } \\
\hline & \begin{tabular}{|c|}
$\begin{array}{c}\text { Number } \\
\text { of } \\
\text { years }\end{array}$ \\
\end{tabular} & $\begin{array}{c}\text { Error } \\
\pm\end{array}$ & & \begin{tabular}{|c|}
$\begin{array}{c}\text { Number } \\
\text { of } \\
\text { years }\end{array}$ \\
\end{tabular} & $\begin{array}{c}\text { Error } \\
\pm\end{array}$ & & \begin{tabular}{|c|}
$\begin{array}{c}\text { Number } \\
\text { of } \\
\text { years }\end{array}$ \\
\end{tabular} & $\begin{array}{c}\text { Error } \\
\pm\end{array}$ & & \begin{tabular}{|c|}
$\begin{array}{c}\text { Number } \\
\text { of } \\
\text { years }\end{array}$ \\
\end{tabular} & $\begin{array}{c}\text { Error } \\
\pm\end{array}$ \\
\hline $\begin{array}{l}0.055426 \\
0.055588 \\
0.055750 \\
0.055912 \\
0.056075\end{array}$ & $\begin{array}{l}351 \\
352 \\
353 \\
354 \\
355\end{array}$ & $\begin{array}{l}0.8 \\
0.8 \\
0.8 \\
0.8 \\
0.8\end{array}$ & $\begin{array}{l}0.406671 \\
0.408040 \\
0.409408 \\
0.410780 \\
0.412151\end{array}$ & $\begin{array}{l}351 \\
352 \\
353 \\
354 \\
355\end{array}$ & $\begin{array}{l}7.9 \\
7.9 \\
7.9 \\
7.9 \\
8.0\end{array}$ & $\begin{array}{l}0.053283 \\
0.053307 \\
0.053330 \\
0.053354 \\
0.053376\end{array}$ & $\begin{array}{l}351 \\
352 \\
353 \\
354 \\
355\end{array}$ & $\begin{array}{l}61.4 \\
61.4 \\
61.4 \\
61.4 \\
61.4\end{array}$ & $\begin{array}{l}0.017279 \\
0.017329 \\
0.017379 \\
0.017428 \\
0.017478\end{array}$ & $\begin{array}{l}351 \\
35 ? \\
353 \\
354 \\
355\end{array}$ & $\begin{array}{l}17.3 \\
17.4 \\
17.4 \\
17.5 \\
17.5\end{array}$ \\
\hline $\begin{array}{l}0.056237 \\
0.056399 \\
0.056561 \\
0.056724 \\
0.056887\end{array}$ & $\begin{array}{l}356 \\
357 \\
358 \\
359 \\
360\end{array}$ & $\begin{array}{l}0.8 \\
0.8 \\
0.8 \\
0.8 \\
0.8\end{array}$ & $\begin{array}{l}0.413524 \\
0.414900 \\
0.416276 \\
0.417655 \\
0.419032\end{array}$ & $\begin{array}{l}356 \\
357 \\
358 \\
359 \\
360\end{array}$ & $\begin{array}{l}8.0 \\
8.0 \\
8.0 \\
8.1 \\
8.1\end{array}$ & $\begin{array}{l}0.053400 \\
0.053424 \\
0.053447 \\
0.053470 \\
0.053493\end{array}$ & $\begin{array}{l}356 \\
357 \\
358 \\
359 \\
360\end{array}$ & $\begin{array}{l}61 \\
6] \\
6] \\
6]\end{array}$ & $\begin{array}{l}0,017527 \\
0.017577 \\
0.017627 \\
0.017677 \\
0.017726\end{array}$ & $\begin{array}{l}356 \\
357 \\
359 \\
359 \\
360\end{array}$ & $\begin{array}{l}17.5 \\
17.6 \\
17.6 \\
17.7 \\
17.7\end{array}$ \\
\hline $\begin{array}{l}0.057049 \\
0.057211 \\
0.057374 \\
0.057536 \\
0.057699\end{array}$ & $\begin{array}{l}361 \\
362 \\
363 \\
364 \\
365\end{array}$ & $\begin{array}{l}0.8 \\
0.8 \\
0.8 \\
0.8 \\
0.8\end{array}$ & $\begin{array}{l}0.420413 \\
0.421794 \\
0.423178 \\
0.424562 \\
0.425947\end{array}$ & $\begin{array}{l}361 \\
362 \\
363 \\
364 \\
365\end{array}$ & $\begin{array}{l}8.1 \\
8.1 \\
8.1 \\
8.2 \\
8.2\end{array}$ & $\begin{array}{l}0.053517 \\
0.053541 \\
0.053564 \\
0.053587 \\
0.053610\end{array}$ & $\begin{array}{l}361 \\
362 \\
363 \\
364 \\
365\end{array}$ & $\begin{array}{l}61.6 \\
61.6 \\
61.6 \\
61.6 \\
61.6\end{array}$ & $\begin{array}{l}0.017776 \\
0.017826 \\
0.017875 \\
0.017925 \\
0.017974\end{array}$ & $\begin{array}{l}361 \\
36 ? \\
363 \\
364 \\
365\end{array}$ & $\begin{array}{l}17.8 \\
17.8 \\
17.9 \\
17.9 \\
18.0\end{array}$ \\
\hline $\begin{array}{l}0.057862 \\
0.058023 \\
0.058187 \\
0.058350 \\
0.058511\end{array}$ & $\begin{array}{l}366 \\
367 \\
368 \\
369 \\
370\end{array}$ & $\begin{array}{l}0.8 \\
0.8 \\
0.8 \\
0.8 \\
0.8\end{array}$ & $\begin{array}{l}0.427333 \\
0.428722 \\
0.430110 \\
0.431501 \\
0.432895\end{array}$ & $\begin{array}{l}366 \\
367 \\
368 \\
369 \\
370\end{array}$ & $\begin{array}{l}8.2 \\
8.2 \\
8.3 \\
8.3 \\
8.3\end{array}$ & $\begin{array}{l}633 \\
658 \\
680 \\
704 \\
729\end{array}$ & $\begin{array}{l}366 \\
367 \\
368 \\
369 \\
370\end{array}$ & $\begin{array}{l}61.6 \\
61.7 \\
61.6 \\
61.6 \\
61.7\end{array}$ & $\begin{array}{l}8024 \\
8074 \\
8124 \\
8174 \\
8223\end{array}$ & $\begin{array}{l}366 \\
367 \\
369 \\
369 \\
3 \div 0\end{array}$ & $\begin{array}{l}18.0 \\
18.1 \\
18.1 \\
18.2 \\
18.2\end{array}$ \\
\hline $\begin{array}{l}0.058674 \\
0.058838 \\
0.059001 \\
0.059162 \\
0.059325\end{array}$ & $\begin{array}{l}371 \\
372 \\
373 \\
374 \\
375\end{array}$ & $\begin{array}{l}0.8 \\
0.8 \\
0.8 \\
0.8 \\
0.8\end{array}$ & $\begin{array}{l}0.43 \\
0.43 \\
0.43 \\
0.43 \\
0.43\end{array}$ & $\begin{array}{l}371 \\
372 \\
373 \\
374 \\
375\end{array}$ & $\begin{array}{l}8.3 \\
8.3 \\
8.4 \\
8.4 \\
8.4\end{array}$ & $\begin{array}{l}752 \\
774 \\
798 \\
823 \\
846\end{array}$ & $\begin{array}{l}371 \\
372 \\
373 \\
374 \\
375\end{array}$ & $\begin{array}{l}61.7 \\
61.6 \\
61.7 \\
61.8 \\
61.8\end{array}$ & $\begin{array}{l}3272 \\
3322 \\
3373 \\
3423 \\
3473\end{array}$ & 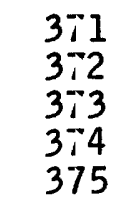 & $\begin{array}{l}18.3 \\
18.3 \\
18.4 \\
18.4 \\
18.5\end{array}$ \\
\hline $\begin{array}{l}0.059489 \\
0.059651 \\
0.059814 \\
0.059977 \\
0.060140\end{array}$ & $\begin{array}{l}376 \\
377 \\
378 \\
379 \\
380\end{array}$ & $\begin{array}{l}0.8 \\
0.8 \\
0.8 \\
0.8 \\
0.8\end{array}$ & $\begin{array}{l}0.441277 \\
0.442680 \\
0.444082 \\
0.445486 \\
0.446892\end{array}$ & $\begin{array}{l}376 \\
377 \\
378 \\
379 \\
380\end{array}$ & $\begin{array}{l}8.4 \\
8.5 \\
8.5 \\
8.5 \\
8.5\end{array}$ & $\begin{array}{l}869 \\
899 \\
917 \\
9940 \\
964\end{array}$ & $\begin{array}{l}376 \\
377 \\
378 \\
379 \\
380\end{array}$ & $\begin{array}{l}61.8 \\
61.8 \\
61.9 \\
61.8 \\
61.8\end{array}$ & $\begin{array}{l}522 \\
572 \\
3622 \\
672 \\
3722\end{array}$ & $\begin{array}{l}376 \\
377 \\
378 \\
379 \\
380\end{array}$ & $\begin{array}{l}18.5 \\
18.6 \\
18.6 \\
18.7 \\
18.7\end{array}$ \\
\hline $\begin{array}{l}0.060303 \\
0.060466 \\
0.060628 \\
0.060792 \\
0.060955\end{array}$ & $\begin{array}{l}381 \\
382 \\
383 \\
384 \\
385\end{array}$ & $\begin{array}{l}0.8 \\
0.8 \\
0.8 \\
0.9 \\
0.9\end{array}$ & $\begin{array}{l}0.448301 \\
0.449708 \\
0.451119 \\
0.452530 \\
0.453943\end{array}$ & $\begin{array}{l}381 \\
382 \\
383 \\
384 \\
385\end{array}$ & $\begin{array}{l}8.5 \\
8.6 \\
8.6 \\
8.6 \\
8.6\end{array}$ & $\begin{array}{l}0.053987 \\
0.054011 \\
0.054036 \\
0.054058 \\
0.054082\end{array}$ & $\begin{array}{l}381 \\
382 \\
383 \\
384 \\
385\end{array}$ & $\begin{array}{l}61.9 \\
61.9 \\
61.9 \\
61.9 \\
61.9\end{array}$ & $\begin{array}{l}0.018770 \\
0.018820 \\
0.018870 \\
0.018920 \\
0.018970\end{array}$ & $\begin{array}{l}381 \\
38 ? \\
383 \\
384 \\
385\end{array}$ & $\begin{array}{l}18.8 \\
18.8 \\
18.9 \\
18.9 \\
19.0\end{array}$ \\
\hline $\begin{array}{l}0.061118 \\
0.061281 \\
0.061444 \\
0.061608 \\
0.061770\end{array}$ & $\begin{array}{l}386 \\
387 \\
388 \\
389 \\
390\end{array}$ & $\begin{array}{l}0.9 \\
0.9 \\
0.9 \\
0.9 \\
0.9\end{array}$ & $\begin{array}{l}0.455356 \\
0.456771 \\
0.458190 \\
0.459609 \\
0.461028\end{array}$ & $\begin{array}{l}386 \\
387 \\
388 \\
389 \\
390\end{array}$ & $\begin{array}{l}8.7 \\
8.7 \\
8.7 \\
8.7 \\
8.8\end{array}$ & $\begin{array}{l}0.054106 \\
0.054130 \\
0.054154 \\
0.054177 \\
0.054202\end{array}$ & $\begin{array}{l}386 \\
387 \\
388 \\
389 \\
390\end{array}$ & $\begin{array}{l}62.0 \\
62.0 \\
62.0 \\
62.0 \\
62.0\end{array}$ & $\begin{array}{l}0.019019 \\
0.019069 \\
0.019119 \\
0.019169 \\
0.019219\end{array}$ & $\begin{array}{l}386 \\
387 \\
388 \\
389 \\
390\end{array}$ & $\begin{array}{l}19.0 \\
19.1 \\
19.1 \\
19.2 \\
19.2\end{array}$ \\
\hline $\begin{array}{l}0.061934 \\
0.062097 \\
0.062260 \\
0.062423 \\
0.062587\end{array}$ & $\begin{array}{l}391 \\
392 \\
393 \\
394 \\
395\end{array}$ & $\begin{array}{l}0.9 \\
0.9 \\
0.9 \\
0.9 \\
0.9\end{array}$ & $\begin{array}{l}0.462449 \\
0.463870 \\
0.465294 \\
0.466718 \\
0.468147\end{array}$ & $\begin{array}{l}391 \\
392 \\
393 \\
394 \\
395\end{array}$ & $\begin{array}{l}8.8 \\
8.8 \\
8.8 \\
8.8 \\
8.9\end{array}$ & $\begin{array}{l}0.054225 \\
0.054248 \\
0.054273 \\
0.054297 \\
0.054320\end{array}$ & $\begin{array}{l}391 \\
392 \\
393 \\
394 \\
395\end{array}$ & $\begin{array}{l}62.0 \\
62.0 \\
62.1 \\
62.1 \\
62.1\end{array}$ & $\begin{array}{l}0.019269 \\
0.019318 \\
0.019367 \\
0.019417 \\
0.019467\end{array}$ & $\begin{array}{l}391 \\
392 \\
393 \\
394 \\
395\end{array}$ & $\begin{array}{l}19.3 \\
19.3 \\
19.4 \\
19.4 \\
19.5\end{array}$ \\
\hline $\begin{array}{l}0.062750 \\
0.062913 \\
0.063077 \\
0.063241 \\
0.063403\end{array}$ & $\begin{array}{l}396 \\
397 \\
398 \\
399 \\
400\end{array}$ & $\begin{array}{l}0.9 \\
0.9 \\
0.9 \\
0.9 \\
0.9\end{array}$ & $\begin{array}{l}0.469575 \\
0.471004 \\
0.472435 \\
0.473866 \\
0.475300\end{array}$ & $\begin{array}{l}396 \\
397 \\
398 \\
399 \\
400\end{array}$ & $\begin{array}{l}8.9 \\
8.9 \\
8.9 \\
9.0 \\
9.0\end{array}$ & $\begin{array}{l}0.054344 \\
0.054368 \\
0.054392 \\
0.054415 \\
0.054440\end{array}$ & $\begin{array}{l}396 \\
397 \\
398 \\
399 \\
400\end{array}$ & $\begin{array}{l}62.1 \\
62.2 \\
62.2 \\
62.2 \\
62.2\end{array}$ & $\begin{array}{l}0.019517 \\
0.019566 \\
0.019616 \\
0.019666 \\
0.019716\end{array}$ & $\begin{array}{l}396 \\
397 \\
398 \\
399 \\
400\end{array}$ & $\begin{array}{l}19.5 \\
19.6 \\
19.6 \\
19.7 \\
19.7\end{array}$ \\
\hline
\end{tabular}


Geologic age, in millions of years, calculated from the atomic ratios of indicated isotopes

\begin{tabular}{|c|c|c|c|c|c|c|c|c|c|c|c|}
\hline \multicolumn{3}{|c|}{$N_{206} / N_{238}$} & \multicolumn{3}{|c|}{$N_{207} / N_{235}$} & \multicolumn{3}{|c|}{$N_{207} / N_{206}$} & \multicolumn{3}{|c|}{$N_{208} / N_{232}$} \\
\hline \multirow[b]{2}{*}{ Ratio } & \multicolumn{2}{|c|}{ Age } & \multirow[b]{2}{*}{ Ratio } & \multicolumn{2}{|c|}{ Age } & \multirow[b]{2}{*}{ Ratio } & \multicolumn{2}{|c|}{ Age } & \multirow[b]{2}{*}{ Ratio } & \multicolumn{2}{|c|}{$\mathrm{Ag} \circ$} \\
\hline & \begin{tabular}{|c|}
$\begin{array}{c}\text { Number } \\
\text { of } \\
\text { years }\end{array}$ \\
\end{tabular} & $\begin{array}{c}\text { Error } \\
\pm\end{array}$ & & \begin{tabular}{|c|}
$\begin{array}{c}\text { Number } \\
\text { of } \\
\text { years }\end{array}$ \\
\end{tabular} & $\begin{array}{c}\text { Error } \\
\pm\end{array}$ & & \begin{tabular}{|c|}
$\begin{array}{c}\text { Number } \\
\text { of } \\
\text { years }\end{array}$ \\
\end{tabular} & $\begin{array}{c}\text { Error } \\
\pm \\
\end{array}$ & & \begin{tabular}{|c|}
$\begin{array}{c}\text { Number } \\
\text { of } \\
\text { years }\end{array}$ \\
\end{tabular} & $\begin{array}{c}\text { Error } \\
\pm\end{array}$ \\
\hline $\begin{array}{l}0.063568 \\
0.063731 \\
0.063894 \\
0.064057 \\
0.064222\end{array}$ & $\begin{array}{l}401 \\
402 \\
403 \\
404 \\
405\end{array}$ & $\begin{array}{l}0.9 \\
0.9 \\
0.9 \\
0.9\end{array}$ & $\begin{array}{l}0.476737 \\
0.478171 \\
0.479608 \\
0.481049 \\
0.482489\end{array}$ & $\begin{array}{l}401 \\
402 \\
403 \\
404 \\
405\end{array}$ & $\begin{array}{l}9.0 \\
9.0 \\
9.0 \\
9.1 \\
9.1\end{array}$ & $\begin{array}{l}0.054463 \\
0.054487 \\
0.054512 \\
0.054536 \\
0.054559\end{array}$ & $\begin{array}{l}401 \\
402 \\
403 \\
404 \\
405\end{array}$ & $\begin{array}{l}62.2 \\
62.2 \\
62.3 \\
62.3 \\
62.3\end{array}$ & $\begin{array}{l}0.019766 \\
0.019815 \\
0.019865 \\
0.019915 \\
0.019965\end{array}$ & $\begin{array}{l}401 \\
402 \\
403 \\
404 \\
405\end{array}$ & $\begin{array}{l}19.8 \\
19.8 \\
19.9 \\
19.9 \\
20.0\end{array}$ \\
\hline $\begin{array}{l}0.064384 \\
0.064548 \\
0.064713 \\
0.064875 \\
0.065039\end{array}$ & $\begin{array}{l}406 \\
407 \\
408 \\
409 \\
410\end{array}$ & $\begin{array}{l}0.9 \\
0.9 \\
0.9 \\
0.9 \\
0.9\end{array}$ & $\begin{array}{l}0.483930 \\
0.485374 \\
0.486820 \\
0.488265 \\
0.489712\end{array}$ & $\begin{array}{l}406 \\
407 \\
408 \\
409 \\
410\end{array}$ & $\begin{array}{l}9.1 \\
9.1 \\
9.2 \\
9.2 \\
9.2\end{array}$ & $\begin{array}{l}0.054584 \\
0.054608 \\
0.054631 \\
0.054656 \\
0.054680\end{array}$ & $\begin{array}{l}406 \\
407 \\
408 \\
409 \\
410\end{array}$ & $\begin{array}{l}62.3 \\
62.4 \\
62.3 \\
62.4 \\
62.4\end{array}$ & $\begin{array}{l}0.020015 \\
0.020065 \\
0.020114 \\
0.020164 \\
0.020214\end{array}$ & $\begin{array}{l}406 \\
407 \\
408 \\
409 \\
410\end{array}$ & $\begin{array}{l}20.0 \\
20.1 \\
20.1 \\
20.2 \\
20.2\end{array}$ \\
\hline $\begin{array}{l}0.065204 \\
0.065367 \\
0.065530 \\
0.065694 \\
0.065859\end{array}$ & $\begin{array}{l}411 \\
412 \\
413 \\
414 \\
415\end{array}$ & $\begin{array}{l}0.9 \\
0.9 \\
0.9 \\
0.9 \\
0.9\end{array}$ & $\begin{array}{l}0.491160 \\
0.492610 \\
0.494063 \\
0.495516 \\
0.496971\end{array}$ & $\begin{array}{l}411 \\
412 \\
413 \\
414 \\
415\end{array}$ & $\begin{array}{l}9.2 \\
9.2 \\
9.3 \\
9.3 \\
9.3\end{array}$ & $\begin{array}{l}0.054703 \\
0.054728 \\
0.054753 \\
0.054776 \\
0.054800\end{array}$ & $\begin{array}{l}411 \\
412 \\
413 \\
414 \\
415\end{array}$ & $\begin{array}{l}62.3 \\
62.4 \\
62.5 \\
62.4 \\
62.5\end{array}$ & $\begin{array}{l}0.020264 \\
0.020314 \\
0.020363 \\
0.020413 \\
0.020463\end{array}$ & $\begin{array}{l}411 \\
412 \\
413 \\
414 \\
415\end{array}$ & $\begin{array}{l}20.3 \\
20.3 \\
20.4 \\
20.4 \\
20.5\end{array}$ \\
\hline $\begin{array}{l}0.066021 \\
0.066185 \\
0.066350 \\
0.066513 \\
0.066677\end{array}$ & $\begin{array}{l}416 \\
417 \\
418 \\
419 \\
420\end{array}$ & $\begin{array}{l}0.9 \\
0.9 \\
0.9 \\
0.9 \\
0.9\end{array}$ & $\begin{array}{l}0.498427 \\
0.499885 \\
0.501344 \\
0.502804 \\
0.504267\end{array}$ & $\begin{array}{l}416 \\
417 \\
418 \\
419 \\
420\end{array}$ & $\begin{array}{l}9.3 \\
9.4 \\
9.4 \\
9.4 \\
9.4\end{array}$ & $\begin{array}{l}0.054825 \\
0.054849 \\
0.054873 \\
0.054898 \\
0.054922\end{array}$ & $\begin{array}{l}416 \\
417 \\
418 \\
419 \\
420\end{array}$ & $\begin{array}{l}62.5 \\
62.5 \\
62.5 \\
62.6 \\
62.6\end{array}$ & $\begin{array}{l}0.020513 \\
0.020563 \\
0.020612 \\
0.020662 \\
0.020712\end{array}$ & $\begin{array}{l}416 \\
417 \\
418 \\
419 \\
420\end{array}$ & $\begin{array}{l}20.5 \\
20.6 \\
20.6 \\
20.7 \\
20.7\end{array}$ \\
\hline $\begin{array}{l}066842 \\
067005 \\
067169 \\
067334 \\
067498\end{array}$ & $\begin{array}{l}421 \\
422 \\
423 \\
424 \\
425\end{array}$ & $\begin{array}{l}0.9 \\
0.9 \\
0.9 \\
0.9 \\
0.9\end{array}$ & $\begin{array}{l}0.505729 \\
0.507192 \\
0.508653 \\
0.510126 \\
0.511595\end{array}$ & $\begin{array}{l}421 \\
422 \\
423 \\
424 \\
425\end{array}$ & $\begin{array}{l}9.4 \\
9.5 \\
9.5 \\
9.5 \\
9.5\end{array}$ & $\begin{array}{l}0.054945 \\
0.054970 \\
0.054994 \\
0.055018 \\
0.055042\end{array}$ & $\begin{array}{l}421 \\
422 \\
423 \\
424 \\
425\end{array}$ & $\begin{array}{l}62.5 \\
62.6 \\
62.6 \\
62.6 \\
62.6\end{array}$ & $\begin{array}{l}0.020762 \\
0.020812 \\
0.020862 \\
0.020911 \\
0.020961\end{array}$ & $\begin{array}{l}421 \\
422 \\
423 \\
424 \\
425\end{array}$ & $\begin{array}{l}20 \\
20 \\
20 \\
20 \\
21\end{array}$ \\
\hline $\begin{array}{l}0.067661 \\
0.067826 \\
0.067990 \\
0.068153 \\
0.068318\end{array}$ & $\begin{array}{l}426 \\
427 \\
428 \\
429 \\
430\end{array}$ & $\begin{array}{l}0.9 \\
0.9 \\
0.9 \\
1.0 \\
1.0\end{array}$ & $\begin{array}{l}0.513065 \\
0.514537 \\
0.516009 \\
0.517485 \\
0.518960\end{array}$ & $\begin{array}{l}426 \\
427 \\
428 \\
429 \\
430\end{array}$ & $\begin{array}{l}9.6 \\
9.6 \\
9.6 \\
9.6 \\
9.6\end{array}$ & $\begin{array}{l}068 \\
091 \\
116 \\
141 \\
165\end{array}$ & $\begin{array}{l}426 \\
427 \\
428 \\
429 \\
430\end{array}$ & $\begin{array}{l}62.7 \\
62.7 \\
62.7 \\
62.7\end{array}$ & $\begin{array}{l}0.021011 \\
0.021061 \\
0.021111 \\
0.021160 \\
0.021210\end{array}$ & $\begin{array}{l}426 \\
427 \\
428 \\
429 \\
430\end{array}$ & $\begin{array}{l}21.0 \\
21.0 \\
21.1 \\
21.1 \\
21.2\end{array}$ \\
\hline $\begin{array}{l}0.068482 \\
0.068646 \\
0.068811 \\
0.068975 \\
0.069138\end{array}$ & $\begin{array}{l}431 \\
432 \\
433 \\
434 \\
435\end{array}$ & $\begin{array}{l}1.0 \\
1.0 \\
1.0 \\
1.0 \\
1.0\end{array}$ & $\begin{array}{l}0.520437 \\
0.521917 \\
0.523398 \\
0.524878 \\
0.526361\end{array}$ & $\begin{array}{l}431 \\
432 \\
433 \\
434 \\
435\end{array}$ & $\begin{array}{l}9.7 \\
9.7 \\
9.7 \\
9.7 \\
9.8\end{array}$ & $\begin{array}{l}0.055189 \\
0.055214 \\
0.055238 \\
0.055262 \\
0.055288\end{array}$ & $\begin{array}{l}431 \\
432 \\
433 \\
434 \\
435\end{array}$ & $\begin{array}{l}62.7 \\
62.8 \\
62.8 \\
62.8\end{array}$ & $\begin{array}{l}0.021260 \\
0.021310 \\
0.021360 \\
0.021410 \\
0.021459\end{array}$ & $\begin{array}{l}431 \\
432 \\
433 \\
434 \\
435\end{array}$ & $\begin{array}{l}21.2 \\
21.3 \\
21.3 \\
21.4 \\
21.4\end{array}$ \\
\hline $\begin{array}{l}0.069304 \\
0.069468 \\
0.069632 \\
0.069797 \\
0.069961\end{array}$ & $\begin{array}{l}436 \\
437 \\
438 \\
439 \\
440\end{array}$ & $\begin{array}{l}1.0 \\
1.0 \\
1.0 \\
1.0 \\
1.0\end{array}$ & $\begin{array}{l}0.527845 \\
0.529333 \\
0.530821 \\
0.532309 \\
0.533800\end{array}$ & $\begin{array}{l}436 \\
437 \\
438\end{array}$ & $\begin{array}{l}9.8 \\
9.8 \\
9.8 \\
9.9 \\
9.9\end{array}$ & $\begin{array}{l}0.055311 \\
0.055336 \\
0.055361 \\
0.055385 \\
0.055410\end{array}$ & $\begin{array}{l}436 \\
437 \\
438 \\
439 \\
440\end{array}$ & $\begin{array}{l}62.8 \\
62.8 \\
62.9 \\
62.9 \\
62.9\end{array}$ & $\begin{array}{l}0.021509 \\
0.021559 \\
0.021609 \\
0.021659 \\
0.021708\end{array}$ & $\begin{array}{l}436 \\
437 \\
438 \\
439 \\
440\end{array}$ & $\begin{array}{l}21 \\
21 \\
21 \\
21 \\
21\end{array}$ \\
\hline $\begin{array}{l}0.070125 \\
0.070290 \\
0.070454 \\
0.070619 \\
0.070783\end{array}$ & $\begin{array}{l}441 \\
442 \\
443 \\
444 \\
445\end{array}$ & $\begin{array}{l}1.0 \\
1.0 \\
1.0 \\
1.0 \\
1.0\end{array}$ & $\begin{array}{l}0.535291 \\
0.536784 \\
0.538278 \\
0.539774 \\
0.541275\end{array}$ & $\begin{array}{l}441 \\
442 \\
443 \\
444 \\
445\end{array}$ & $\begin{array}{r}9.9 \\
9.9 \\
9.9 \\
10.0 \\
10.0\end{array}$ & $\begin{array}{l}0.055434 \\
0.055459 \\
0.055483 \\
0.055508 \\
0.055533\end{array}$ & $\begin{array}{l}441 \\
442 \\
443 \\
444 \\
445\end{array}$ & $\begin{array}{l}62.9 \\
62.9 \\
62.9 \\
63.0 \\
63.0\end{array}$ & $\begin{array}{l}0.021758 \\
0.021808 \\
0.021858 \\
0.021908 \\
0.021957\end{array}$ & $\begin{array}{l}441 \\
442 \\
443 \\
444 \\
445\end{array}$ & $\begin{array}{l}21.7 \\
21.8 \\
21.8 \\
21.9 \\
21.9\end{array}$ \\
\hline $\begin{array}{l}0.070948 \\
0.071113 \\
0.071277 \\
0.071441 \\
0.071607\end{array}$ & $\begin{array}{l}446 \\
447 \\
448 \\
449 \\
450\end{array}$ & $\begin{array}{l}1.0 \\
1.0 \\
1.0 \\
1.0 \\
1.0\end{array}$ & $\begin{array}{l}0.542773 \\
0.544273 \\
0.545774 \\
0.547277 \\
0.548782\end{array}$ & $\begin{array}{l}446 \\
447 \\
448 \\
449 \\
450\end{array}$ & $\begin{array}{l}100 \\
10.0 \\
10.1 \\
10.1 \\
10.1\end{array}$ & $\begin{array}{l}0.055557 \\
0.055581 \\
0.055607 \\
0.055632 \\
0.055655\end{array}$ & $\begin{array}{l}446 \\
447 \\
448 \\
449 \\
450\end{array}$ & $\begin{array}{l}63.0 \\
63.0 \\
63.1 \\
63.1 \\
63.0\end{array}$ & $\begin{array}{l}0.022007 \\
0.022058 \\
0.022108 \\
0.022158 \\
0.022208\end{array}$ & $\begin{array}{l}446 \\
447 \\
448 \\
449 \\
450\end{array}$ & $\begin{array}{l}22.0 \\
22.0 \\
22.1 \\
22.1 \\
22.1\end{array}$ \\
\hline
\end{tabular}


Geologic age, in millions of years, calculated from the atomic ratios of indicated isotopes

\begin{tabular}{|c|c|c|c|c|c|c|c|c|c|c|c|}
\hline \multicolumn{3}{|c|}{$N_{206} / N_{238}$} & \multicolumn{3}{|c|}{$N_{207} / N_{235}$} & \multicolumn{3}{|c|}{$N_{207} / N_{206}$} & \multicolumn{3}{|c|}{$N_{208} / N_{232}$} \\
\hline \multirow[b]{2}{*}{ Ratio } & \multicolumn{2}{|c|}{ Age } & \multirow[b]{2}{*}{ Ratio } & \multicolumn{2}{|c|}{ Age } & \multirow[b]{2}{*}{ Ratio } & \multicolumn{2}{|c|}{ Age } & \multirow[b]{2}{*}{ Ratio } & \multicolumn{2}{|c|}{ Age } \\
\hline & $\begin{array}{c}\text { Number } \\
\text { of } \\
\text { years }\end{array}$ & $\begin{array}{c}\text { Error } \\
\pm\end{array}$ & & \begin{tabular}{|c|}
$\begin{array}{c}\text { Number } \\
\text { of } \\
\text { years }\end{array}$ \\
\end{tabular} & $\begin{array}{c}\text { Error } \\
\pm\end{array}$ & & \begin{tabular}{|c|}
$\begin{array}{c}\text { Number } \\
\text { of } \\
\text { years }\end{array}$ \\
\end{tabular} & $\begin{array}{c}\text { Error } \\
\pm\end{array}$ & & \begin{tabular}{|c|}
$\begin{array}{c}\text { Number } \\
\text { of } \\
\text { years }\end{array}$ \\
\end{tabular} & $\begin{array}{c}\text { Error } \\
\pm\end{array}$ \\
\hline $\begin{array}{l}0.071771 \\
0.071935 \\
0.072101 \\
0.072266 \\
0.072430\end{array}$ & $\begin{array}{l}451 \\
452 \\
453 \\
454 \\
455\end{array}$ & $\begin{array}{l}1.0 \\
1.0 \\
1.0 \\
1.0 \\
1.0\end{array}$ & $\begin{array}{l}0.550289 \\
0.551797 \\
0.553307 \\
0.554818 \\
0.556330\end{array}$ & $\begin{array}{l}451 \\
452 \\
453 \\
454 \\
455\end{array}$ & $\begin{array}{l}10.1 \\
10.1 \\
10.2 \\
10.2 \\
10.2\end{array}$ & $\begin{array}{l}0.055681 \\
0.055706 \\
0.055730 \\
0.055754 \\
0.055780\end{array}$ & $\begin{array}{l}451 \\
452 \\
453 \\
454 \\
455\end{array}$ & $\begin{array}{l}63.1 \\
63.1 \\
63.1 \\
63.1 \\
63.2\end{array}$ & $\begin{array}{l}0.022257 \\
0.022307 \\
0.022357 \\
0.022407 \\
0.022457\end{array}$ & $\begin{array}{l}451 \\
452 \\
453 \\
454 \\
455\end{array}$ & $\begin{array}{l}22.2 \\
22.3 \\
22.3 \\
22.4 \\
22.4\end{array}$ \\
\hline $\begin{array}{l}0.072595 \\
0.072761 \\
0.072926 \\
0.073091 \\
0.073256\end{array}$ & $\begin{array}{l}456 \\
457 \\
458 \\
459 \\
460\end{array}$ & $\begin{array}{l}1.0 \\
1.0 \\
1.0 \\
1.0 \\
1.0\end{array}$ & $\begin{array}{l}0.557843 \\
0.559360 \\
0.560875 \\
0.562392 \\
0.563913\end{array}$ & $\begin{array}{l}456 \\
457 \\
458 \\
459 \\
460\end{array}$ & $\begin{array}{l}10.2 \\
10.3 \\
10.3 \\
10.3 \\
10.3\end{array}$ & $\begin{array}{l}0.055804 \\
0.055828 \\
0.055853 \\
0.055878 \\
0.055902\end{array}$ & $\begin{array}{l}456 \\
457 \\
458 \\
459 \\
460\end{array}$ & $\begin{array}{l}63.2 \\
63.1 \\
63.2 \\
63.2 \\
63.2\end{array}$ & $\begin{array}{l}0.022506 \\
0.022556 \\
0.022606 \\
0.022656 \\
0.022707\end{array}$ & $\begin{array}{l}456 \\
457 \\
458 \\
459 \\
460\end{array}$ & $\begin{array}{l}22.5 \\
22.5 \\
22.6 \\
22.6 \\
22.7\end{array}$ \\
\hline $\begin{array}{l}0.073420 \\
0.073585 \\
0.073751 \\
0.073915 \\
0.074080\end{array}$ & $\begin{array}{l}461 \\
462 \\
463 \\
464 \\
465\end{array}$ & $\begin{array}{l}1.0 \\
1.0 \\
1.0 \\
1.0 \\
1.0\end{array}$ & $\begin{array}{l}0.565433 \\
0.566957 \\
0.568481 \\
0.570007 \\
0.571533\end{array}$ & $\begin{array}{l}461 \\
462 \\
463 \\
464 \\
465\end{array}$ & $\begin{array}{l}10.3 \\
10.4 \\
10.4 \\
10.4 \\
10.4\end{array}$ & $\begin{array}{l}0.055928 \\
0.055953 \\
0.055977 \\
0.056003 \\
0.056028\end{array}$ & $\begin{array}{l}461 \\
462 \\
463 \\
464 \\
465\end{array}$ & $\begin{array}{l}63.2 \\
63.3 \\
63.2 \\
63.3 \\
63.3\end{array}$ & $\begin{array}{l}0.022756 \\
0.022806 \\
0.022856 \\
0.022906 \\
0.022956\end{array}$ & $\begin{array}{l}461 \\
462 \\
463 \\
464 \\
465\end{array}$ & $\begin{array}{l}22.7 \\
22.8 \\
22.8 \\
22.9 \\
22.9\end{array}$ \\
\hline $\begin{array}{l}0.074246 \\
0.074411 \\
0.074576 \\
0.074741 \\
0.074906\end{array}$ & $\begin{array}{l}466 \\
467 \\
468 \\
469 \\
470\end{array}$ & $\begin{array}{l}1.0 \\
1.0 \\
1.0 \\
1.0 \\
1.0\end{array}$ & $\begin{array}{l}0.573060 \\
0.574592 \\
0.576123 \\
0.577656 \\
0.579191\end{array}$ & $\begin{array}{l}466 \\
467 \\
468 \\
469 \\
470\end{array}$ & $\begin{array}{l}10.5 \\
10.5 \\
10.5 \\
10.5 \\
10.5\end{array}$ & $\begin{array}{l}0.056052 \\
0.056077 \\
0.056102 \\
0.056127 \\
0.056152\end{array}$ & $\begin{array}{l}466 \\
467 \\
468 \\
469 \\
470\end{array}$ & $\begin{array}{l}63.3 \\
63.3 \\
63.3 \\
63.4 \\
63.4\end{array}$ & $\begin{array}{l}3006 \\
3055 \\
3105 \\
3156 \\
3207\end{array}$ & $\begin{array}{l}466 \\
467 \\
468 \\
469 \\
470\end{array}$ & $\begin{array}{l}23.0 \\
23.0 \\
23.1 \\
23.1 \\
23.2\end{array}$ \\
\hline $\begin{array}{l}0.075072 \\
0.075237 \\
0.075403 \\
0.075567 \\
0.075732\end{array}$ & $\begin{array}{l}471 \\
472 \\
473 \\
474 \\
475\end{array}$ & $\begin{array}{l}1.0 \\
1.0 \\
1.0 \\
1.1 \\
1.1\end{array}$ & $\begin{array}{l}0.580727 \\
0.582264 \\
0.583803 \\
0.585343 \\
0.586885\end{array}$ & $\begin{array}{l}471 \\
472 \\
473 \\
474 \\
475\end{array}$ & $\begin{array}{l}10.6 \\
10.6 \\
10.6 \\
10.6 \\
10.7\end{array}$ & $\begin{array}{l}0.056177 \\
0.056202 \\
0.056226 \\
0.056252 \\
0.056278\end{array}$ & $\begin{array}{l}471 \\
472 \\
473 \\
474 \\
475\end{array}$ & $\begin{array}{l}63.4 \\
63.4 \\
63.4 \\
63.4 \\
63.5\end{array}$ & $\begin{array}{l}0.023257 \\
0.023306 \\
0.023356 \\
0.023406 \\
0.023456\end{array}$ & $\begin{array}{l}471 \\
472 \\
473 \\
474 \\
475\end{array}$ & $\begin{array}{l}23.2 \\
23.3 \\
23.3 \\
23.4 \\
23.4\end{array}$ \\
\hline $\begin{array}{l}0.075898 \\
0.076063 \\
0.076229 \\
0.076394 \\
0.076560\end{array}$ & $\begin{array}{l}476 \\
477 \\
478 \\
479 \\
480\end{array}$ & $\begin{array}{l}1.1 \\
1.1 \\
1.1 \\
1.1 \\
1.1\end{array}$ & $\begin{array}{l}0.588429 \\
0.589974 \\
0.591520 \\
0.593069 \\
0.594617\end{array}$ & $\begin{array}{l}476 \\
477 \\
478 \\
479 \\
480\end{array}$ & $\begin{array}{l}10.7 \\
10.7 \\
10.7 \\
10.7 \\
10.8\end{array}$ & $\begin{array}{l}0.056302 \\
0.056328 \\
0.056352 \\
0.056378 \\
0.056402\end{array}$ & $\begin{array}{l}476 \\
477 \\
478 \\
479 \\
480\end{array}$ & $\begin{array}{l}63.5 \\
63.5 \\
63.5 \\
63.5 \\
63.5\end{array}$ & $\begin{array}{l}0.023506 \\
0.023556 \\
0.023606 \\
0.023656 \\
0.023706\end{array}$ & $\begin{array}{l}476 \\
477 \\
478 \\
479 \\
480\end{array}$ & $\begin{array}{l}23.5 \\
23.5 \\
23.6 \\
23.6 \\
23.7\end{array}$ \\
\hline $\begin{array}{l}0.076725 \\
0.076890 \\
0.077057 \\
0.077221 \\
0.077388\end{array}$ & $\begin{array}{l}481 \\
482 \\
483 \\
484 \\
485\end{array}$ & $\begin{array}{l}1.1 \\
1.1 \\
1.1 \\
1.1 \\
1.1\end{array}$ & $\begin{array}{l}0.596168 \\
0.597722 \\
0.599276 \\
0.600832 \\
0.602389\end{array}$ & $\begin{array}{l}481 \\
482 \\
483 \\
484 \\
485\end{array}$ & $\begin{array}{l}10.8 \\
10.8 \\
10.8 \\
10.9 \\
10.9\end{array}$ & $\begin{array}{l}0.056428 \\
0.056454 \\
0.056478 \\
0.056504 \\
0.056528\end{array}$ & $\begin{array}{l}481 \\
482 \\
483 \\
484 \\
485\end{array}$ & $\begin{array}{l}63.6 \\
63.6 \\
63.6 \\
63.6 \\
63.6\end{array}$ & $\begin{array}{l}0.023756 \\
0.023806 \\
0.023855 \\
0.023906 \\
0.023956\end{array}$ & $\begin{array}{l}481 \\
482 \\
483 \\
484 \\
485\end{array}$ & $\begin{array}{l}23.7 \\
23.8 \\
23.8 \\
23.9 \\
23.9\end{array}$ \\
\hline $\begin{array}{l}0.077553 \\
0.077718 \\
0.077884 \\
0.078050 \\
0.078215\end{array}$ & $\begin{array}{l}486 \\
487 \\
488 \\
489 \\
490\end{array}$ & $\begin{array}{l}1.1 \\
1.1 \\
1.1 \\
1.1 \\
1.1\end{array}$ & $\begin{array}{l}0.603947 \\
0.605507 \\
0.607069 \\
0.608632 \\
0.610197\end{array}$ & $\begin{array}{l}486 \\
487 \\
488 \\
489 \\
490\end{array}$ & $\begin{array}{l}10.9 \\
10.9 \\
11.0 \\
11.0 \\
11.0\end{array}$ & $\begin{array}{l}0.056554 \\
0.056580 \\
0.056605 \\
0.056630 \\
0.056656\end{array}$ & $\begin{array}{l}486 \\
487 \\
488 \\
489 \\
490\end{array}$ & $\begin{array}{l}63.7 \\
63.7 \\
63.7 \\
63.7 \\
63.8\end{array}$ & $\begin{array}{l}0.024006 \\
0.024056 \\
0.024105 \\
0.024155 \\
0.024206\end{array}$ & $\begin{array}{l}486 \\
487 \\
488 \\
489 \\
490\end{array}$ & $\begin{array}{l}24.0 \\
24.0 \\
24.1 \\
24.1 \\
24.2\end{array}$ \\
\hline $\begin{array}{l}0.078381 \\
0.078547 \\
0.078713 \\
0.078878 \\
0.079044\end{array}$ & $\begin{array}{l}491 \\
492 \\
493 \\
494 \\
495\end{array}$ & $\begin{array}{l}1.1 \\
1.1 \\
1.1 \\
1.1 \\
1.1\end{array}$ & $\begin{array}{l}0.611761 \\
0.613330 \\
0.614898 \\
0.616469 \\
0.618043\end{array}$ & $\begin{array}{l}491 \\
492 \\
493 \\
494 \\
495\end{array}$ & $\begin{array}{l}11.0 \\
11.0 \\
11.1 \\
11.1 \\
11.1\end{array}$ & $\begin{array}{l}0.056680 \\
0.056706 \\
0.056731 \\
0.056757 \\
0.056782\end{array}$ & $\begin{array}{l}491 \\
492 \\
493 \\
494 \\
495\end{array}$ & $\begin{array}{l}63.7 \\
63.8 \\
63.8 \\
63.8 \\
63.8\end{array}$ & $\begin{array}{l}0.024256 \\
0.024306 \\
0.024355 \\
0.024405 \\
0.024455\end{array}$ & $\begin{array}{l}491 \\
492 \\
493 \\
494 \\
495\end{array}$ & $\begin{array}{l}24.2 \\
24.3 \\
24.3 \\
24.4 \\
24.4\end{array}$ \\
\hline $\begin{array}{l}079210 \\
0.079376 \\
0.079542 \\
0079708 \\
0.079873\end{array}$ & $\begin{array}{l}496 \\
497 \\
498 \\
499 \\
500\end{array}$ & $\begin{array}{l}1.1 \\
1.1 \\
1.1 \\
1.1 \\
1.1\end{array}$ & $\begin{array}{l}0.619617 \\
0.621191 \\
0.622767 \\
0.624346 \\
0.625927\end{array}$ & $\begin{array}{l}496 \\
497 \\
498 \\
499 \\
500\end{array}$ & $\begin{array}{l}11.1 \\
11.2 \\
11.2 \\
11.2 \\
11.2\end{array}$ & $\begin{array}{l}0.056807 \\
0.056833 \\
0.056858 \\
0.056883 \\
0.056910\end{array}$ & $\begin{array}{l}496 \\
497 \\
498 \\
499 \\
500\end{array}$ & $\begin{array}{l}63.8 \\
63.9 \\
63.9 \\
63.9 \\
63.9\end{array}$ & $\begin{array}{l}0.024506 \\
0.024556 \\
0.024606 \\
0.024655 \\
0.024705\end{array}$ & $\begin{array}{l}496 \\
497 \\
498 \\
499 \\
500\end{array}$ & $\begin{array}{l}24.5 \\
24.5 \\
24.5 \\
24.6 \\
24.6\end{array}$ \\
\hline
\end{tabular}


Geologic age, in millions of years, calculated from the atomic ratios of indicated isotopes

\begin{tabular}{|c|c|c|c|c|c|c|c|c|c|c|c|}
\hline \multicolumn{3}{|c|}{$N_{206} / N_{238}$} & \multicolumn{3}{|c|}{$N_{207} / N_{235}$} & \multicolumn{3}{|c|}{$N_{207} / N_{206}$} & \multicolumn{3}{|c|}{$N_{208} / N_{232}$} \\
\hline \multirow[b]{2}{*}{ Ratio } & \multicolumn{2}{|c|}{ Age } & \multirow[b]{2}{*}{ Ratio } & \multicolumn{2}{|c|}{ Age } & \multirow[b]{2}{*}{ Ratio } & \multicolumn{2}{|c|}{ Age } & \multirow[b]{2}{*}{ Ratio } & \multicolumn{2}{|c|}{ Age } \\
\hline & \begin{tabular}{|c|}
$\begin{array}{c}\text { Number } \\
\text { of } \\
\text { years }\end{array}$ \\
\end{tabular} & $\begin{array}{c}\text { Error } \\
\pm\end{array}$ & & \begin{tabular}{|c|} 
Number \\
of \\
years
\end{tabular} & $\begin{array}{c}\text { Error } \\
\pm\end{array}$ & & \begin{tabular}{|c|}
$\begin{array}{c}\text { Number } \\
\text { of } \\
\text { years }\end{array}$ \\
\end{tabular} & $\begin{array}{c}\text { Error } \\
\pm \\
\end{array}$ & & \begin{tabular}{|c|}
$\begin{array}{c}\text { Number } \\
\text { of } \\
\text { years }\end{array}$ \\
\end{tabular} & $\begin{array}{c}\text { Error } \\
\pm\end{array}$ \\
\hline $\begin{array}{l}0.080206 \\
0.080538 \\
0.080870 \\
0.081202 \\
0.081534\end{array}$ & $\begin{array}{l}502 \\
504 \\
506 \\
508 \\
510\end{array}$ & $\begin{array}{l}1.1 \\
1.1 \\
1.1 \\
1.1 \\
1.1\end{array}$ & $\begin{array}{l}0.629090 \\
0.632262 \\
0.635436 \\
0.638621 \\
0.641809\end{array}$ & $\begin{array}{l}502 \\
504 \\
506 \\
508 \\
510\end{array}$ & $\begin{array}{l}11.3 \\
11.3 \\
11.4 \\
11.4 \\
11.4\end{array}$ & $\begin{array}{l}0.056960 \\
0.057011 \\
0.057062 \\
0.057113 \\
0.057165\end{array}$ & $\begin{array}{l}502 \\
504 \\
506 \\
508 \\
510\end{array}$ & $\begin{array}{l}63.9 \\
64.0 \\
64.0 \\
64.0 \\
64.1\end{array}$ & $\begin{array}{l}0.024806 \\
0.024905 \\
0.025006 \\
0.025106 \\
0.025205\end{array}$ & $\begin{array}{l}502 \\
504 \\
506 \\
508 \\
510\end{array}$ & $\begin{array}{l}24.7 \\
24.8 \\
24.9 \\
25.0 \\
25.1\end{array}$ \\
\hline $\begin{array}{l}0.081868 \\
0.082200 \\
0.082532 \\
0.082866 \\
0.083198\end{array}$ & $\begin{array}{l}512 \\
514 \\
516 \\
518 \\
520\end{array}$ & $\begin{array}{l}1.1 \\
1.1 \\
1.1 \\
1.1 \\
1.2\end{array}$ & $\begin{array}{l}0.645005 \\
0.648207 \\
0.651415 \\
0.654627 \\
0.657850\end{array}$ & $\begin{array}{l}512 \\
514 \\
516 \\
518 \\
520\end{array}$ & $\begin{array}{l}11.5 \\
11.5 \\
11.6 \\
11.6 \\
11.7\end{array}$ & $\begin{array}{l}0.057215 \\
0.057267 \\
0.057319 \\
0.057369 \\
0.057422\end{array}$ & $\begin{array}{l}512 \\
514 \\
516 \\
518 \\
520\end{array}$ & $\begin{array}{l}64.1 \\
64.2 \\
64.2 \\
64.2 \\
64.2\end{array}$ & $\begin{array}{l}0.025306 \\
0.025406 \\
0.025506 \\
0.025606 \\
0.025706\end{array}$ & $\begin{array}{l}512 \\
514 \\
516 \\
518 \\
520\end{array}$ & $\begin{array}{l}25.3 \\
25.4 \\
25.5 \\
25.6\end{array}$ \\
\hline $\begin{array}{l}0.083532 \\
0.083864 \\
0.084197 \\
0.084531 \\
0.084864\end{array}$ & $\begin{array}{l}522 \\
524 \\
526 \\
528 \\
530\end{array}$ & $\begin{array}{l}1.2 \\
1.2 \\
1.2 \\
1.2 \\
1.2\end{array}$ & $\begin{array}{l}0.661075 \\
0.664306 \\
0.667547 \\
0.670792 \\
0.674043\end{array}$ & $\begin{array}{l}522 \\
524 \\
526 \\
528 \\
530\end{array}$ & $\begin{array}{l}11.7 \\
11.8 \\
11.8 \\
11.8 \\
11.9\end{array}$ & $\begin{array}{l}0.057473 \\
0.057525 \\
0.057577 \\
0.057628 \\
0.057680\end{array}$ & $\begin{array}{l}522 \\
524 \\
526 \\
528 \\
530\end{array}$ & $\begin{array}{l}64.3 \\
64.3 \\
64.4 \\
64.3 \\
64.4\end{array}$ & $\begin{array}{l}0.025806 \\
0.025907 \\
0.026006 \\
0.026107 \\
0.026207\end{array}$ & $\begin{array}{l}522 \\
524 \\
526 \\
528 \\
530\end{array}$ & $\begin{array}{l}25.7 \\
25.8 \\
25.9 \\
26.0 \\
26.1\end{array}$ \\
\hline $\begin{array}{l}0.085198 \\
0.085531 \\
0.085865 \\
0.086199 \\
0.086532\end{array}$ & $\begin{array}{l}532 \\
534 \\
536 \\
538 \\
540\end{array}$ & $\begin{array}{l}1.2 \\
1.2 \\
1.2 \\
1.2 \\
1.2\end{array}$ & $\begin{array}{l}0.677303 \\
0.680568 \\
0.683837 \\
0.687116 \\
0.690399\end{array}$ & $\begin{array}{l}532 \\
534 \\
536 \\
538 \\
540\end{array}$ & $\begin{array}{l}11.9 \\
12.0 \\
12.0 \\
12.1 \\
12.1\end{array}$ & $\begin{array}{l}0.057732 \\
0.057784 \\
0.057836 \\
0.057888 \\
0.057941\end{array}$ & $\begin{array}{l}532 \\
534 \\
536 \\
538 \\
540\end{array}$ & $\begin{array}{l}64.4 \\
64.5 \\
64.5 \\
64.6 \\
64.6\end{array}$ & $\begin{array}{l}0.026307 \\
0.026407 \\
0.026507 \\
0.026608 \\
0.026709\end{array}$ & $\begin{array}{l}532 \\
534 \\
536 \\
538 \\
540\end{array}$ & $\begin{array}{l}26.2 \\
26.3 \\
26.4 \\
26.5 \\
26.6\end{array}$ \\
\hline $\begin{array}{l}0.086866 \\
0.087202 \\
0.087535 \\
0.087870 \\
0.088204\end{array}$ & $\begin{array}{l}542 \\
544 \\
546 \\
548 \\
550\end{array}$ & $\begin{array}{l}1.2 \\
1.2 \\
1.2 \\
1.2 \\
1.2\end{array}$ & $\begin{array}{l}0.693688 \\
0.696984 \\
0.700288 \\
0.703595 \\
0.706911\end{array}$ & $\begin{array}{l}542 \\
544 \\
546 \\
548 \\
550\end{array}$ & $\begin{array}{l}12.2 \\
12.2 \\
12.3 \\
12.3 \\
12.3\end{array}$ & $\begin{array}{l}0.057993 \\
0.058044 \\
0.058097 \\
0.058149 \\
0.058202\end{array}$ & $\begin{array}{l}542 \\
544 \\
546 \\
548 \\
550\end{array}$ & $\begin{array}{l}64.7 \\
64.6 \\
64.7 \\
64.7 \\
64.8\end{array}$ & $\begin{array}{l}808 \\
909 \\
010 \\
109 \\
210\end{array}$ & $\begin{array}{l}542 \\
544 \\
546 \\
548 \\
550\end{array}$ & $\begin{array}{l}26.7 \\
26.8 \\
26.9 \\
27.0 \\
27.1\end{array}$ \\
\hline $\begin{array}{l}0.088538 \\
0.088873 \\
0.089208 \\
0.089544 \\
0.089878\end{array}$ & $\begin{array}{l}552 \\
554 \\
556 \\
558 \\
560\end{array}$ & $\begin{array}{l}1.2 \\
1.2 \\
1.2 \\
1.2 \\
1.2\end{array}$ & $\begin{array}{l}0.710233 \\
0.713561 \\
0.716895 \\
0.720240 \\
0.723585\end{array}$ & $\begin{array}{l}552 \\
554 \\
556 \\
558 \\
560\end{array}$ & $\begin{array}{l}12.4 \\
12.4 \\
12.5 \\
12.5 \\
12.6\end{array}$ & $\begin{array}{l}255 \\
307 \\
360 \\
412 \\
465\end{array}$ & $\begin{array}{l}552 \\
554 \\
556 \\
558 \\
560\end{array}$ & $\begin{array}{l}64.8 \\
64.8 \\
64.9 \\
64.9 \\
64.9\end{array}$ & $\begin{array}{l}309 \\
4110 \\
511 \\
610 \\
711\end{array}$ & $\begin{array}{l}552 \\
554 \\
556 \\
558 \\
560\end{array}$ & $\begin{array}{l}27.2 \\
27.3 \\
27.4 \\
27.5 \\
27.6\end{array}$ \\
\hline $\begin{array}{l}0.090213 \\
0.090548 \\
0.090883 \\
0.091218 \\
0.091555\end{array}$ & $\begin{array}{l}562 \\
564 \\
566 \\
568 \\
570\end{array}$ & $\begin{array}{l}1.2 \\
1.3 \\
1.3 \\
1.3 \\
1.3\end{array}$ & $\begin{array}{l}0.726939 \\
0.730301 \\
0.733669 \\
0.737042 \\
0.740425\end{array}$ & $\begin{array}{l}562 \\
564 \\
566 \\
568 \\
570\end{array}$ & $\begin{array}{l}12.6 \\
12.7 \\
12.7 \\
12.7 \\
12.8\end{array}$ & $\begin{array}{l}0.058518 \\
0.058571 \\
0.058625 \\
0.058678 \\
0.058730\end{array}$ & $\begin{array}{l}562 \\
564 \\
566 \\
568 \\
570\end{array}$ & $\begin{array}{l}65.0 \\
65.0 \\
65.1 \\
65.1 \\
65.1\end{array}$ & $\begin{array}{l}0.027812 \\
0.027911 \\
0.028012 \\
0.028112 \\
0.028213\end{array}$ & $\begin{array}{l}562 \\
564 \\
566 \\
568 \\
570\end{array}$ & $\begin{array}{l}27.7 \\
27.8 \\
27.9 \\
28.0 \\
28.1\end{array}$ \\
\hline $\begin{array}{l}0.091890 \\
0.092226 \\
0.092561 \\
0.092896 \\
0.093234\end{array}$ & $\begin{array}{l}572 \\
574 \\
576 \\
578 \\
580\end{array}$ & $\begin{array}{l}1.3 \\
1.3 \\
1.3 \\
1.3 \\
1.3\end{array}$ & $\begin{array}{l}0.743810 \\
0.747203 \\
0.750605 \\
0.754012 \\
0.757425\end{array}$ & $\begin{array}{l}572 \\
574 \\
576 \\
578 \\
580\end{array}$ & $\begin{array}{l}12.8 \\
12.9 \\
12.9 \\
13.0 \\
13.0\end{array}$ & $\begin{array}{l}0.058784 \\
0.058837 \\
0.058891 \\
0.058945 \\
0.058997\end{array}$ & $\begin{array}{l}572 \\
574 \\
576 \\
578 \\
580\end{array}$ & $\begin{array}{l}65.1 \\
65.2 \\
65.2 \\
65.3 \\
65.3\end{array}$ & $\begin{array}{l}0.028313 \\
0.028413 \\
0.028514 \\
0.028615 \\
0.028714\end{array}$ & $\begin{array}{l}572 \\
574 \\
576 \\
578 \\
580\end{array}$ & $\begin{array}{l}28.2 \\
28.3 \\
28.4 \\
28.5 \\
28.6\end{array}$ \\
\hline $\begin{array}{l}0.093569 \\
0.09390 \\
0.094241 \\
0.094577 \\
0.094915\end{array}$ & $\begin{array}{l}582 \\
584 \\
586 \\
588 \\
590\end{array}$ & $\begin{array}{l}1.3 \\
1.3 \\
1.3 \\
1.3 \\
1.3\end{array}$ & $\begin{array}{l}0.760847 \\
0.764272 \\
0.767706 \\
0.771147 \\
0.774595\end{array}$ & $\begin{array}{l}582 \\
584 \\
586 \\
588 \\
590\end{array}$ & $\begin{array}{l}13.1 \\
13.1 \\
13.2 \\
13.2 \\
13.2\end{array}$ & $\begin{array}{l}0.059051 \\
0.059104 \\
0.059159 \\
0.059213 \\
0.059266\end{array}$ & $\begin{array}{l}582 \\
584 \\
586 \\
588 \\
590\end{array}$ & $\begin{array}{l}65.3 \\
65.3 \\
65.4 \\
65.5 \\
65.5\end{array}$ & $\begin{array}{l}0.028815 \\
0.028916 \\
0.029016 \\
0.029116 \\
0.029216\end{array}$ & $\begin{array}{l}582 \\
584 \\
586 \\
588 \\
590\end{array}$ & $\begin{array}{l}28.7 \\
28.8 \\
28.9 \\
29.0 \\
29.1\end{array}$ \\
\hline $\begin{array}{l}0.095251 \\
0.095587 \\
0.095925 \\
0.096261 \\
0.096598\end{array}$ & $\begin{array}{l}592 \\
594 \\
596 \\
598 \\
600\end{array}$ & $\begin{array}{l}1.3 \\
1.3 \\
1.3 \\
1.3 \\
1.3\end{array}$ & $\begin{array}{l}0.778049 \\
0.781509 \\
0.784976 \\
0.788450 \\
0.791929\end{array}$ & $\begin{array}{l}592 \\
594 \\
596 \\
598 \\
600\end{array}$ & $\begin{array}{l}13.3 \\
13.3 \\
13.4 \\
13.4 \\
13.5\end{array}$ & $\begin{array}{l}0.059320 \\
0.059374 \\
0.059427 \\
0.059482 \\
0.059536\end{array}$ & $\begin{array}{l}592 \\
594 \\
596 \\
598 \\
600\end{array}$ & $\begin{array}{l}65.5 \\
65.5 \\
65.6 \\
65.6 \\
65.6\end{array}$ & $\begin{array}{l}0.029318 \\
0.029419 \\
0.029519 \\
0.029620 \\
0.029720\end{array}$ & $\begin{array}{l}592 \\
594 \\
596 \\
598 \\
600\end{array}$ & $\begin{array}{l}29 \\
29 \\
29 \\
29 \\
29\end{array}$ \\
\hline
\end{tabular}


Geologic age, in millions of years, calculated from the atomic ratios of indicated isotopes

\begin{tabular}{|c|c|c|c|c|c|c|c|c|c|c|c|}
\hline \multicolumn{3}{|c|}{$N_{206} / N_{238}$} & \multicolumn{3}{|c|}{$N_{207} / N_{235}$} & \multicolumn{3}{|c|}{$N_{207} / N_{206}$} & \multicolumn{3}{|c|}{$N_{208} / N_{232}$} \\
\hline \multirow[b]{2}{*}{ Ratio } & \multicolumn{2}{|c|}{ Age } & \multirow[b]{2}{*}{ Ratio } & \multicolumn{2}{|c|}{ Age } & \multirow[b]{2}{*}{ Ratio } & \multicolumn{2}{|c|}{ Age } & \multirow[b]{2}{*}{ Ratio } & \multicolumn{2}{|c|}{ Age } \\
\hline & $\begin{array}{c}\begin{array}{c}\text { Number } \\
\text { of } \\
\text { years }\end{array} \\
\end{array}$ & $\begin{array}{c}\text { Error } \\
\pm\end{array}$ & & \begin{tabular}{|c|}
$\begin{array}{c}\text { Number } \\
\text { of } \\
\text { years }\end{array}$ \\
\end{tabular} & $\begin{array}{c}\text { Error } \\
\pm\end{array}$ & & $\begin{array}{c}\text { Number } \\
\text { of } \\
\text { years }\end{array}$ & $\begin{array}{c}\text { Error } \\
\pm\end{array}$ & & \begin{tabular}{|c|}
$\begin{array}{c}\text { Number } \\
\text { of } \\
\text { years }\end{array}$ \\
\end{tabular} & $\begin{array}{c}\text { Error } \\
\pm\end{array}$ \\
\hline $\begin{array}{l}0.096936 \\
0.097273 \\
0.097611 \\
0.097947 \\
0.098285\end{array}$ & $\begin{array}{l}602 \\
604 \\
606 \\
608 \\
610\end{array}$ & $\begin{array}{l}1.3 \\
1.3 \\
1.3 \\
1.3 \\
1.4\end{array}$ & $\begin{array}{l}0.795417 \\
0.798911 \\
0.802413 \\
0.805921 \\
0.809436\end{array}$ & $\begin{array}{l}602 \\
604 \\
606 \\
608 \\
610\end{array}$ & $\begin{array}{l}13.5 \\
13.6 \\
13.6 \\
13.6 \\
13.7\end{array}$ & $\begin{array}{l}590 \\
644 \\
698 \\
754 \\
808\end{array}$ & $\begin{array}{l}602 \\
604 \\
606 \\
608 \\
610\end{array}$ & $\begin{array}{l}6 \\
6 \\
6\end{array}$ & $\begin{array}{l}820 \\
921 \\
021 \\
122 \\
223\end{array}$ & $\begin{array}{l}602 \\
604 \\
606 \\
608 \\
610\end{array}$ & $\begin{array}{l}29.7 \\
29.8 \\
29.9 \\
30.0 \\
30.1\end{array}$ \\
\hline $\begin{array}{l}0.098623 \\
0.098961 \\
0.099299 \\
0.099636 \\
0.099974\end{array}$ & $\begin{array}{l}612 \\
614 \\
616 \\
618 \\
620\end{array}$ & $\begin{array}{l}1.4 \\
1.4 \\
1.4 \\
1.4 \\
1.4\end{array}$ & $\begin{array}{l}0.812957 \\
0.816486 \\
0.820020 \\
0.823562 \\
0.827113\end{array}$ & $\begin{array}{l}612 \\
614 \\
616 \\
618 \\
620\end{array}$ & $\begin{array}{l}13.7 \\
13.8 \\
13.8 \\
13.9 \\
13.9\end{array}$ & $\begin{array}{l}862 \\
917 \\
971 \\
026 \\
081\end{array}$ & $\begin{array}{l}612 \\
614 \\
616 \\
618 \\
620\end{array}$ & & $\begin{array}{l}0.030323 \\
0.030424 \\
0.030525 \\
0.030624 \\
0.030725\end{array}$ & $\begin{array}{l}612 \\
614 \\
616 \\
618 \\
620\end{array}$ & $\begin{array}{l}30.2 \\
30.3 \\
30.4 \\
30.5 \\
30.6\end{array}$ \\
\hline $\begin{array}{l}0.100313 \\
0.100650 \\
0,100990 \\
0.101327 \\
0.101666\end{array}$ & $\begin{array}{l}622 \\
624 \\
626 \\
628 \\
630\end{array}$ & $\begin{array}{l}1.4 \\
1.4 \\
1.4 \\
1.4 \\
1.4\end{array}$ & $\begin{array}{l}0,830667 \\
0.834231 \\
0.837801 \\
0.841377 \\
0.844961\end{array}$ & $\begin{array}{l}622 \\
624 \\
626 \\
628 \\
630\end{array}$ & $\begin{array}{l}14.0 \\
14.0 \\
14.0 \\
14.1 \\
14.1\end{array}$ & $\begin{array}{l}136 \\
191 \\
246 \\
301 \\
356\end{array}$ & $\begin{array}{l}622 \\
624 \\
626 \\
628 \\
630\end{array}$ & 6 & $\begin{array}{l}825 \\
926 \\
027 \\
127 \\
228\end{array}$ & $\begin{array}{l}622 \\
624 \\
626 \\
628 \\
630\end{array}$ & $\begin{array}{l}30.7 \\
30.8 \\
30.9 \\
31.0 \\
31.1\end{array}$ \\
\hline $\begin{array}{l}0.102005 \\
0.102344 \\
0.102682 \\
0.103022 \\
0.103360\end{array}$ & $\begin{array}{l}632 \\
634 \\
636 \\
638 \\
640\end{array}$ & $\begin{array}{l}1.4 \\
1.4 \\
1.4 \\
1.4 \\
1.4\end{array}$ & $\begin{array}{l}552 \\
150 \\
754 \\
365 \\
985\end{array}$ & $\begin{array}{l}632 \\
634 \\
636 \\
638 \\
640\end{array}$ & $\begin{array}{l}14.2 \\
14.2 \\
14.3 \\
14.3 \\
14.4\end{array}$ & $\begin{array}{l}411 \\
467 \\
523 \\
577 \\
634\end{array}$ & $\begin{array}{l}632 \\
634 \\
636 \\
638 \\
640\end{array}$ & 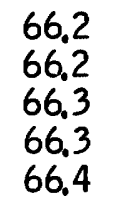 & $\begin{array}{l}329 \\
429 \\
530 \\
631 \\
732\end{array}$ & $\begin{array}{l}632 \\
634 \\
636 \\
638 \\
640\end{array}$ & $\begin{array}{l}31.2 \\
31.3 \\
31.4 \\
31.5 \\
31.5\end{array}$ \\
\hline $\begin{array}{l}0.103700 \\
0.104039 \\
0.104379 \\
0.104719 \\
0.105058\end{array}$ & $\begin{array}{l}642 \\
644 \\
646 \\
648 \\
650\end{array}$ & $\begin{array}{l}1.4 \\
1.4 \\
1.4 \\
1.4 \\
1.4\end{array}$ & $\begin{array}{l}611 \\
242 \\
884 \\
529 \\
183\end{array}$ & $\begin{array}{l}642 \\
644 \\
646 \\
648 \\
650\end{array}$ & $\begin{array}{l}14.4 \\
14.5 \\
14.5 \\
14.5 \\
14.6\end{array}$ & $\begin{array}{l}689 \\
744 \\
800 \\
855 \\
912\end{array}$ & $\begin{array}{l}642 \\
644 \\
646 \\
648 \\
650\end{array}$ & 6 & $\begin{array}{l}334 \\
934 \\
35 \\
36 \\
36\end{array}$ & $\begin{array}{l}642 \\
644 \\
646 \\
648 \\
650\end{array}$ & $\begin{array}{l}31.6 \\
31.7 \\
31.8 \\
31.9 \\
32.0\end{array}$ \\
\hline $\begin{array}{l}0.105398 \\
0.105738 \\
0.106077 \\
0.106418 \\
0.106757\end{array}$ & $\begin{array}{l}652 \\
654 \\
656 \\
658 \\
660\end{array}$ & $\begin{array}{l}1.4 \\
1.5 \\
1.5 \\
1.5 \\
1.5\end{array}$ & $\begin{array}{l}7846 \\
3513 \\
189 \\
872 \\
5611\end{array}$ & $\begin{array}{l}652 \\
654 \\
656 \\
658 \\
660\end{array}$ & $\begin{array}{l}14.6 \\
14.7 \\
14.7 \\
14.8 \\
14.8\end{array}$ & $\begin{array}{l}967 \\
023 \\
080 \\
135 \\
192\end{array}$ & $\begin{array}{l}652 \\
654 \\
656 \\
658 \\
660\end{array}$ & & $\begin{array}{l}337 \\
437 \\
538 \\
639 \\
739\end{array}$ & $\begin{array}{l}652 \\
654 \\
656 \\
658 \\
650\end{array}$ & $\begin{array}{l}32.2 \\
32.3 \\
32.4 \\
32.5\end{array}$ \\
\hline $\begin{array}{l}0.107097 \\
0.107439 \\
0.107778 \\
0.108120 \\
0.108459\end{array}$ & $\begin{array}{l}662 \\
664 \\
666 \\
668 \\
670\end{array}$ & $\begin{array}{l}1.5 \\
1.5 \\
1.5 \\
1.5 \\
1.5\end{array}$ & $\begin{array}{l}257 \\
963 \\
673 \\
392 \\
117\end{array}$ & $\begin{array}{l}662 \\
664 \\
666 \\
668 \\
670\end{array}$ & $\begin{array}{l}14.9 \\
14.9 \\
14.9 \\
15.0 \\
15.0\end{array}$ & $\begin{array}{l}249 \\
304 \\
361 \\
417 \\
474\end{array}$ & $\begin{array}{l}662 \\
664 \\
666 \\
668 \\
670\end{array}$ & & $\begin{array}{l}841 \\
942 \\
042 \\
143 \\
243\end{array}$ & $\begin{array}{l}652 \\
654 \\
656 \\
658 \\
670\end{array}$ & $\begin{array}{l}32.6 \\
32.7 \\
32.8 \\
32.9 \\
33.0\end{array}$ \\
\hline $\begin{array}{l}0.108800 \\
0.109142 \\
0.109482 \\
0.109824 \\
0.110165\end{array}$ & $\begin{array}{l}672 \\
674 \\
676 \\
678 \\
680\end{array}$ & $\begin{array}{l}1.5 \\
1.5 \\
1.5 \\
1.5 \\
1.5\end{array}$ & $\begin{array}{l}850 \\
591 \\
339 \\
094 \\
855\end{array}$ & $\begin{array}{l}672 \\
674 \\
676 \\
678 \\
680\end{array}$ & $\begin{array}{l}15.1 \\
15.1 \\
15.2 \\
15.2 \\
15.3\end{array}$ & $\begin{array}{l}531 \\
587 \\
644 \\
701 \\
758\end{array}$ & $\begin{array}{l}672 \\
674 \\
676 \\
678 \\
680\end{array}$ & $\begin{array}{l}66.9 \\
66.9 \\
67.0 \\
67.0 \\
67.0\end{array}$ & $\begin{array}{l}0.033344 \\
0.033446 \\
0.033546 \\
0.033648 \\
0.033749\end{array}$ & $\begin{array}{l}672 \\
674 \\
676 \\
678 \\
690\end{array}$ & $\begin{array}{l}33.1 \\
33.2 \\
33.3 \\
33.4 \\
33.5\end{array}$ \\
\hline $\begin{array}{l}0.110506 \\
0.110848 \\
0.111189 \\
0.111531 \\
0.111872\end{array}$ & $\begin{array}{l}682 \\
684 \\
686 \\
688 \\
690\end{array}$ & $\begin{array}{l}1.5 \\
1.5 \\
1.5 \\
1.5 \\
1.5\end{array}$ & $\begin{array}{l}0.940627 \\
0.944403 \\
0,948186 \\
0.951979 \\
0.955778\end{array}$ & $\begin{array}{l}682 \\
684 \\
686 \\
688 \\
690\end{array}$ & $\begin{array}{l}15.3 \\
15.3 \\
15.4 \\
15.4 \\
15.5\end{array}$ & $\begin{array}{l}0.061815 \\
0.061872 \\
0.061929 \\
0.061986 \\
0.062044\end{array}$ & $\begin{array}{l}682 \\
684 \\
686 \\
688 \\
690\end{array}$ & $\begin{array}{l}67.1 \\
67.1 \\
67.1 \\
67.2 \\
67.2\end{array}$ & $\begin{array}{l}0.033850 \\
0.033951 \\
0.034051 \\
0.034152 \\
0.034254\end{array}$ & $\begin{array}{l}682 \\
694 \\
696 \\
698 \\
690\end{array}$ & $\begin{array}{l}33.6 \\
33.7 \\
33.8 \\
33.9 \\
34.0\end{array}$ \\
\hline $\begin{array}{l}0.112214 \\
0.112557 \\
0.112898 \\
0.113240 \\
0.113583\end{array}$ & $\begin{array}{l}692 \\
694 \\
696 \\
698 \\
700\end{array}$ & $\begin{array}{l}1.5 \\
1.5 \\
1.5 \\
1.5 \\
1.6\end{array}$ & $\begin{array}{l}0.959585 \\
0.963397 \\
0.967220 \\
0.971047 \\
0.974883\end{array}$ & $\begin{array}{l}692 \\
694 \\
696 \\
698 \\
700\end{array}$ & $\begin{array}{l}15.5 \\
15.6 \\
15.6 \\
15.7 \\
15.7\end{array}$ & $\begin{array}{l}0.062101 \\
0.062158 \\
0.062216 \\
0.062273 \\
0.062331\end{array}$ & $\begin{array}{l}692 \\
694 \\
696 \\
698 \\
700\end{array}$ & $\begin{array}{l}67.3 \\
67.3 \\
67.3 \\
67.3 \\
67.4\end{array}$ & $\begin{array}{l}0.034354 \\
0.034455 \\
0.034557 \\
0.034657 \\
0.034758\end{array}$ & $\begin{array}{l}692 \\
694 \\
696 \\
698 \\
700\end{array}$ & $\begin{array}{l}34.1 \\
34.2 \\
34.3 \\
34.4 \\
34.5\end{array}$ \\
\hline
\end{tabular}


Geologic age, in millions of years, calculated from the atomic ratios of indicated isotopes

\begin{tabular}{|c|c|c|c|c|c|c|c|c|c|c|c|}
\hline \multicolumn{3}{|c|}{$N_{206} / N_{238}$} & \multicolumn{3}{|c|}{$N_{207} / N_{235}$} & \multicolumn{3}{|c|}{$N_{207} / N_{206}$} & \multicolumn{3}{|c|}{$N_{208} / N_{232}$} \\
\hline \multirow[b]{2}{*}{ Ratio } & \multicolumn{2}{|c|}{ Age } & \multirow[b]{2}{*}{ Ratio } & \multicolumn{2}{|c|}{ Age } & \multirow[b]{2}{*}{ Ratio } & \multicolumn{2}{|c|}{ Age } & \multirow[b]{2}{*}{ Ratio } & \multicolumn{2}{|c|}{ Age } \\
\hline & \begin{tabular}{|c|}
$\begin{array}{c}\text { Number } \\
\text { of } \\
\text { years }\end{array}$ \\
\end{tabular} & $\begin{array}{c}\text { Error } \\
\pm\end{array}$ & & $\begin{array}{c}\text { Number } \\
\text { of } \\
\text { years }\end{array}$ & $\begin{array}{c}\text { Error } \\
\pm\end{array}$ & & $\begin{array}{c}\text { Number } \\
\text { of } \\
\text { years }\end{array}$ & $\begin{array}{c}\text { Error } \\
\pm \\
\end{array}$ & & $\begin{array}{c}\text { Number } \\
\text { of } \\
\text { years }\end{array}$ & $\begin{array}{c}\text { Error } \\
\pm\end{array}$ \\
\hline $\begin{array}{l}0.113925 \\
0.114268 \\
0.114609 \\
0.114952 \\
0.115295\end{array}$ & $\begin{array}{l}702 \\
704 \\
706 \\
708 \\
710\end{array}$ & $\begin{array}{l}1.6 \\
1.6 \\
1.6 \\
1.6 \\
1.6\end{array}$ & $\begin{array}{l}0.978729 \\
0.982578 \\
0.986437 \\
0.990304 \\
0.994176\end{array}$ & $\begin{array}{l}702 \\
704 \\
706 \\
708 \\
710\end{array}$ & $\begin{array}{l}15.8 \\
15.8 \\
15.8 \\
15.9 \\
15.9\end{array}$ & $\begin{array}{l}0.062389 \\
0.062446 \\
0.062505 \\
0.062563 \\
0.062620\end{array}$ & $\begin{array}{l}702 \\
704 \\
706 \\
708 \\
710\end{array}$ & $\begin{array}{l}67.4 \\
67.4 \\
67.5 \\
67.6 \\
67.6\end{array}$ & $\begin{array}{l}0.034859 \\
0.034960 \\
0.035061 \\
0.035162 \\
0.035263\end{array}$ & $\begin{array}{l}702 \\
704 \\
706 \\
708 \\
710\end{array}$ & $\begin{array}{l}34.6 \\
34.7 \\
34.8 \\
34.9 \\
35.0\end{array}$ \\
\hline $\begin{array}{l}0.115638 \\
0.115981 \\
0.116325 \\
0.116667 \\
0.1170_{11}\end{array}$ & $\begin{array}{l}712 \\
714 \\
716 \\
718 \\
720\end{array}$ & $\begin{array}{l}1.6 \\
1.6 \\
1.6 \\
1.6 \\
1.6\end{array}$ & $\begin{array}{l}0.998057 \\
1.001947 \\
1.005842 \\
1.009744 \\
1.013659\end{array}$ & $\begin{array}{l}712 \\
714 \\
716 \\
718 \\
720\end{array}$ & $\begin{array}{l}160 \\
16.0 \\
16.1 \\
16.1 \\
16.2\end{array}$ & $\begin{array}{l}0.062678 \\
0.062737 \\
0.062794 \\
0.062853 \\
0.062911\end{array}$ & $\begin{array}{l}712 \\
714 \\
716 \\
718 \\
720\end{array}$ & $\begin{array}{l}67.6 \\
67.6 \\
67.6 \\
67.7 \\
67.7\end{array}$ & $\begin{array}{l}364 \\
466 \\
567 \\
669 \\
769\end{array}$ & $\begin{array}{l}712 \\
714 \\
716 \\
718 \\
720\end{array}$ & $\begin{array}{l}35.1 \\
35.2 \\
35.3 \\
35.4 \\
35.5\end{array}$ \\
\hline $\begin{array}{l}0.117354 \\
0.117697 \\
0.118042 \\
0.118385 \\
0.118729\end{array}$ & $\begin{array}{l}722 \\
724 \\
726 \\
728 \\
730\end{array}$ & $\begin{array}{l}1.6 \\
1.6 \\
1.6 \\
1.6 \\
1.6\end{array}$ & $\begin{array}{l}1.017577 \\
1.021502 \\
1.025439 \\
1.029379 \\
1.033328\end{array}$ & $\begin{array}{l}722 \\
724 \\
726 \\
728 \\
730\end{array}$ & $\begin{array}{l}162 \\
16.2 \\
16.3 \\
16.3 \\
16.4\end{array}$ & $\begin{array}{l}970 \\
028 \\
086 \\
145 \\
204\end{array}$ & $\begin{array}{l}722 \\
724 \\
726 \\
728 \\
730\end{array}$ & $\begin{array}{l}67.8 \\
67.8 \\
67.8 \\
67.9 \\
67.9\end{array}$ & $\begin{array}{l}871 \\
971 \\
072 \\
174 \\
274\end{array}$ & $\begin{array}{l}722 \\
724 \\
726 \\
728 \\
730\end{array}$ & $\begin{array}{l}35.6 \\
35.7 \\
35.8 \\
35.9 \\
36.0\end{array}$ \\
\hline $\begin{array}{l}0.119073 \\
0.119416 \\
0.119741 \\
0.120105 \\
.120449\end{array}$ & $\begin{array}{l}732 \\
734 \\
736 \\
738 \\
740\end{array}$ & $\begin{array}{l}1.6 \\
1.6 \\
1.6 \\
1.6 \\
1.6\end{array}$ & $\begin{array}{l}1.037288 \\
1.041251 \\
1.045224 \\
1.049203 \\
1.053191\end{array}$ & $\begin{array}{l}732 \\
734 \\
736 \\
738\end{array}$ & $\begin{array}{l}16.4 \\
16.5 \\
16.5 \\
16.6 \\
16.6\end{array}$ & $\begin{array}{l}3263 \\
3322 \\
3381 \\
3440 \\
3499\end{array}$ & $\begin{array}{l}732 \\
734 \\
736 \\
738 \\
740\end{array}$ & $\begin{array}{l}68.0 \\
68.0 \\
68.0 \\
68.1 \\
68.1\end{array}$ & $\begin{array}{l}376 \\
477 \\
578 \\
679 \\
780\end{array}$ & $\begin{array}{l}732 \\
734 \\
736 \\
738 \\
740\end{array}$ & $\begin{array}{l}36.1 \\
36.2 \\
36.3 \\
36.4 \\
36.5\end{array}$ \\
\hline $\begin{array}{l}120795 \\
121139 \\
121484 \\
121829 \\
122173\end{array}$ & $\begin{array}{l}742 \\
744 \\
746 \\
748 \\
750\end{array}$ & $\begin{array}{l}1.6 \\
1.6 \\
1.7 \\
1.7 \\
1.7\end{array}$ & $\begin{array}{l}189 \\
191 \\
203 \\
223 \\
249\end{array}$ & $\begin{array}{l}742 \\
744 \\
746 \\
748 \\
750\end{array}$ & $\begin{array}{l}16.7 \\
16.7 \\
16.7 \\
16.8 \\
16.8\end{array}$ & $\begin{array}{l}557 \\
617 \\
676 \\
735 \\
795\end{array}$ & $\begin{array}{l}742 \\
744 \\
746 \\
748 \\
750\end{array}$ & $\begin{array}{l}1 \\
1 \\
2 \\
2 \\
3\end{array}$ & & $\begin{array}{l}742 \\
744 \\
746 \\
748 \\
750\end{array}$ & $\begin{array}{l}36.6 \\
36.7 \\
36.8 \\
36.9 \\
37.0\end{array}$ \\
\hline $\begin{array}{l}122518 \\
1122863 \\
1123208 \\
0.123553 \\
0.123898\end{array}$ & $\begin{array}{l}752 \\
754 \\
756 \\
758 \\
760\end{array}$ & $\begin{array}{l}1.7 \\
1.7 \\
1.7 \\
1.7 \\
1.7\end{array}$ & $\begin{array}{l}1.077288 \\
1.081328 \\
1.085377 \\
1.089439 \\
1.093504\end{array}$ & $\begin{array}{l}752 \\
754 \\
756 \\
758 \\
760\end{array}$ & $\begin{array}{l}16.9 \\
16.9 \\
170 \\
17.0 \\
17.1\end{array}$ & $\begin{array}{l}855 \\
914 \\
974 \\
034 \\
094\end{array}$ & $\begin{array}{l}752 \\
754 \\
756 \\
758 \\
760\end{array}$ & $\begin{array}{l}68.3 \\
68.3 \\
68.4 \\
68.4 \\
68.4\end{array}$ & & $\begin{array}{l}752 \\
754 \\
756 \\
758 \\
760\end{array}$ & $\begin{array}{l}37.1 \\
37.2 \\
37.3 \\
37.4 \\
37.5\end{array}$ \\
\hline $\begin{array}{l}.124244 \\
124589 \\
1124934 \\
0.125283 \\
0125628\end{array}$ & $\begin{array}{l}762 \\
764 \\
766 \\
768 \\
770\end{array}$ & $\begin{array}{l}1.7 \\
1.7 \\
1.7 \\
1.7 \\
1.7\end{array}$ & $\begin{array}{l}1.097578 \\
1.101661 \\
1.105752 \\
1.109849 \\
1.113957\end{array}$ & $\begin{array}{l}762 \\
764 \\
766 \\
768 \\
770\end{array}$ & $\begin{array}{l}171 \\
17.1 \\
17.2 \\
17.2 \\
17.3\end{array}$ & $\begin{array}{l}0.064154 \\
0.064214 \\
0.064275 \\
0.064333 \\
0.064394\end{array}$ & $\begin{array}{l}762 \\
764 \\
766 \\
768 \\
770\end{array}$ & $\begin{array}{l}68.5 \\
68.5 \\
68.6 \\
68.6 \\
68.6\end{array}$ & $\begin{array}{l}394 \\
996 \\
098 \\
198 \\
300\end{array}$ & $\begin{array}{l}762 \\
764 \\
766 \\
768 \\
770\end{array}$ & $\begin{array}{l}37.6 \\
37.7 \\
37.8 \\
37.9 \\
38.0\end{array}$ \\
\hline $\begin{array}{l}0.125974 \\
0.126320 \\
0.126665 \\
0.127012 \\
0.127359\end{array}$ & $\begin{array}{l}772 \\
774 \\
776 \\
778\end{array}$ & $\begin{array}{l}1.7 \\
1.7 \\
1.7 \\
1.7 \\
1.7\end{array}$ & $\begin{array}{l}1.118071 \\
1.122190 \\
1.126322 \\
1.130459 \\
1.134607\end{array}$ & $\begin{array}{l}772 \\
774 \\
776 \\
778 \\
780\end{array}$ & $\begin{array}{l}17.3 \\
17.4 \\
17.4 \\
17.5 \\
17.5\end{array}$ & $\begin{array}{l}4454 \\
4514 \\
4576 \\
4636 \\
4696\end{array}$ & $\begin{array}{l}772 \\
774 \\
776 \\
778 \\
780\end{array}$ & $\begin{array}{l}68.7 \\
68.7 \\
68.8 \\
68.8 \\
68.8\end{array}$ & $\begin{array}{l}3401 \\
3502 \\
3605 \\
3706 \\
3807\end{array}$ & $\begin{array}{l}772 \\
774 \\
776 \\
778 \\
780\end{array}$ & $\begin{array}{l}38.1 \\
38.2 \\
38.3 \\
38.4 \\
38.5\end{array}$ \\
\hline $\begin{array}{l}0.127705 \\
0.128052 \\
0.128398 \\
0.128746 \\
0.129093\end{array}$ & $\begin{array}{l}782 \\
784 \\
786 \\
788 \\
790\end{array}$ & $\begin{array}{l}1.7 \\
1.7 \\
1.7 \\
1.7 \\
1.8\end{array}$ & $\begin{array}{l}1.138763 \\
1.142923 \\
1.147094 \\
1.151270 \\
1.155459\end{array}$ & $\begin{array}{l}782 \\
784 \\
786 \\
788 \\
790\end{array}$ & $\begin{array}{l}17.5 \\
17.6 \\
17.6 \\
17.7 \\
17.7\end{array}$ & $\begin{array}{l}0.064757 \\
0.064818 \\
0.064879 \\
0.064939 \\
0.065000\end{array}$ & $\begin{array}{l}782 \\
784 \\
786 \\
788 \\
790\end{array}$ & $\begin{array}{l}68.9 \\
68.9 \\
68.9 \\
68.9 \\
69.0\end{array}$ & $\begin{array}{l}909 \\
010 \\
112 \\
212 \\
314\end{array}$ & $\begin{array}{l}782 \\
784 \\
786 \\
788 \\
790\end{array}$ & $\begin{array}{l}38.6 \\
38.7 \\
38.8 \\
38.9\end{array}$ \\
\hline $\begin{array}{l}0.129440 \\
0.129787 \\
0.130135 \\
0.130483 \\
0.130829\end{array}$ & $\begin{array}{l}792 \\
794 \\
796 \\
798 \\
800\end{array}$ & $\begin{array}{l}1.8 \\
1.8 \\
1.8 \\
1.8 \\
1.8\end{array}$ & $\begin{array}{l}1.159653 \\
1.163857 \\
1.168070 \\
1.172287 \\
1.176515\end{array}$ & $\begin{array}{l}792 \\
794 \\
796 \\
798 \\
800\end{array}$ & $\begin{array}{l}17.8 \\
17.8 \\
17.9 \\
17.9 \\
18.0\end{array}$ & $\begin{array}{l}0.065061 \\
0.065123 \\
0.065183 \\
0.065244 \\
0.065306\end{array}$ & $\begin{array}{l}792 \\
794 \\
796 \\
798 \\
800\end{array}$ & $\begin{array}{l}69.0 \\
69.1 \\
69.1 \\
69.1 \\
69.2\end{array}$ & $\begin{array}{l}0.039416 \\
0.039517 \\
0.039618 \\
0.039720 \\
0.039821\end{array}$ & $\begin{array}{l}792 \\
794 \\
796 \\
798 \\
800\end{array}$ & $\begin{array}{l}39.0 \\
39.1 \\
39.2 \\
39.3 \\
39.4\end{array}$ \\
\hline
\end{tabular}


Geologic age, in millions of years, calculated from the atomic ratios of indicated isotopes

\begin{tabular}{|c|c|c|c|c|c|c|c|c|c|c|c|}
\hline \multicolumn{3}{|c|}{$N_{206} / N_{238}$} & \multicolumn{3}{|c|}{$N_{207} / N_{235}$} & \multicolumn{3}{|c|}{$N_{207} / N_{206}$} & \multicolumn{3}{|c|}{$N_{208} / N_{232}$} \\
\hline \multirow[b]{2}{*}{ Ratio } & \multicolumn{2}{|c|}{ Age } & \multirow[b]{2}{*}{ Ratio } & \multicolumn{2}{|c|}{ Age } & \multirow[b]{2}{*}{ Ratio } & \multicolumn{2}{|c|}{ Age } & \multirow[b]{2}{*}{ Ratio } & \multicolumn{2}{|c|}{ Age } \\
\hline & $\begin{array}{c}\begin{array}{c}\text { Number } \\
\text { of } \\
\text { years }\end{array} \\
\end{array}$ & $\begin{array}{c}\text { Error } \\
\pm\end{array}$ & & \begin{tabular}{|c|} 
Number \\
of \\
years
\end{tabular} & $\begin{array}{c}\text { Error } \\
\pm\end{array}$ & & \begin{tabular}{|c|}
$\begin{array}{c}\text { Number } \\
\text { of } \\
\text { years }\end{array}$ \\
\end{tabular} & $\begin{array}{c}\text { Error } \\
\pm \\
\end{array}$ & & $\begin{array}{c}\begin{array}{c}\text { Number } \\
\text { of } \\
\text { years }\end{array} \\
\end{array}$ & $\begin{array}{c}\text { Error } \\
\pm\end{array}$ \\
\hline $\begin{array}{l}0.131177 \\
0.131525 \\
0.131872 \\
0.132220 \\
0.132568\end{array}$ & $\begin{array}{l}802 \\
804 \\
806 \\
808 \\
810\end{array}$ & $\begin{array}{l}1.8 \\
1.8 \\
1.8 \\
1.8 \\
1.8\end{array}$ & $\begin{array}{l}1.180752 \\
1.184996 \\
1.189248 \\
1.193510 \\
1.197777\end{array}$ & $\begin{array}{l}802 \\
804 \\
806 \\
808 \\
810\end{array}$ & $\begin{array}{l}18.0 \\
18.0 \\
18.1 \\
18.1 \\
18.2\end{array}$ & $\begin{array}{l}368 \\
429 \\
491 \\
553 \\
615\end{array}$ & $\begin{array}{l}802 \\
804 \\
806 \\
808 \\
810\end{array}$ & $\begin{array}{l}69.2 \\
69.2 \\
69.3 \\
69.3 \\
69.4\end{array}$ & $\begin{array}{l}923 \\
025 \\
126 \\
228 \\
329\end{array}$ & $\begin{array}{l}802 \\
804 \\
806 \\
808 \\
810\end{array}$ & $\begin{array}{l}39.5 \\
39.6 \\
39.7 \\
39.8 \\
39.9\end{array}$ \\
\hline $\begin{array}{l}0.132917 \\
0.133266 \\
0.133614 \\
0.133962 \\
0.134311\end{array}$ & $\begin{array}{l}812 \\
814 \\
816 \\
818 \\
820\end{array}$ & $\begin{array}{l}1.8 \\
1.8 \\
1.8 \\
1.8 \\
1.8\end{array}$ & $\begin{array}{l}1.202055 \\
1.206341 \\
1.210636 \\
1.214937 \\
1.219249\end{array}$ & $\begin{array}{l}812 \\
814 \\
816 \\
818 \\
820\end{array}$ & $\begin{array}{l}18.2 \\
18.3 \\
18.3 \\
18.4 \\
18.4\end{array}$ & $\begin{array}{l}676 \\
738 \\
800 \\
862 \\
924\end{array}$ & $\begin{array}{l}812 \\
814 \\
816 \\
818 \\
820\end{array}$ & $\begin{array}{l}69.4 \\
69.4 \\
69.5 \\
69.5 \\
69.5\end{array}$ & $\begin{array}{l}31 \\
33 \\
34 \\
36 \\
37\end{array}$ & $\begin{array}{l}812 \\
814 \\
816 \\
818 \\
820\end{array}$ & $\begin{array}{l}40.0 \\
40.1 \\
40.2 \\
40.3 \\
40.4\end{array}$ \\
\hline $\begin{array}{l}0.134660 \\
0.135009 \\
0.135357 \\
0.135706 \\
0.136056\end{array}$ & $\begin{array}{l}822 \\
824 \\
826 \\
828 \\
830\end{array}$ & $\begin{array}{l}1.8 \\
1.8 \\
1.8 \\
1.8 \\
1.8\end{array}$ & $\begin{array}{l}1.223569 \\
1.227894 \\
1.232233 \\
1.236576 \\
1.240929\end{array}$ & $\begin{array}{l}822 \\
824 \\
826 \\
828 \\
830\end{array}$ & $\begin{array}{l}18.4 \\
18.5 \\
18.5 \\
18.6 \\
18.6\end{array}$ & $\begin{array}{l}0.065986 \\
0.066048 \\
0.066111 \\
0.066174 \\
0.066236\end{array}$ & $\begin{array}{l}822 \\
824 \\
826 \\
828 \\
830\end{array}$ & $\begin{array}{l}69.5 \\
69.6 \\
69.6 \\
69.7 \\
69.7\end{array}$ & $\begin{array}{l}0.040938 \\
0.041040 \\
0.041141 \\
0.041243 \\
0.041346\end{array}$ & $\begin{array}{l}822 \\
824 \\
826 \\
828 \\
830\end{array}$ & $\begin{array}{l}40.5 \\
40.6 \\
40.7 \\
40.8 \\
40.9\end{array}$ \\
\hline $\begin{array}{l}0.136405 \\
0.136753 \\
0.137104 \\
0.137453 \\
0.137803\end{array}$ & $\begin{array}{l}832 \\
834 \\
836 \\
838 \\
840\end{array}$ & $\begin{array}{l}1.8 \\
1.8 \\
1.9 \\
1.9 \\
1.9\end{array}$ & $\begin{array}{l}1.245290 \\
1.249659 \\
1.254037 \\
1.258423 \\
1.262819\end{array}$ & $\begin{array}{l}832 \\
834 \\
836 \\
838 \\
840\end{array}$ & $\begin{array}{l}18.7 \\
18.7 \\
18.8 \\
18.8 \\
18.8\end{array}$ & $\begin{array}{l}298 \\
362 \\
424 \\
487 \\
550\end{array}$ & $\begin{array}{l}832 \\
834 \\
836 \\
838 \\
840\end{array}$ & $\begin{array}{l}69.7 \\
69.8 \\
69.8 \\
69.9 \\
69.9\end{array}$ & $\begin{array}{l}47 \\
49 \\
50 \\
52 \\
54\end{array}$ & $\begin{array}{l}832 \\
834 \\
836 \\
838 \\
840\end{array}$ & $\begin{array}{l}41.0 \\
41.1 \\
41.2 \\
41.3 \\
41.4\end{array}$ \\
\hline $\begin{array}{l}0.138153 \\
0.138501 \\
0.138852 \\
0.139201 \\
0.139554\end{array}$ & $\begin{array}{l}842 \\
844 \\
846 \\
848 \\
850\end{array}$ & $\begin{array}{l}1.9 \\
1.9 \\
1.9 \\
1.9 \\
1.9\end{array}$ & $\begin{array}{l}1.267223 \\
1.271635 \\
1.276058 \\
1.280486 \\
1.284925\end{array}$ & $\begin{array}{l}842 \\
844 \\
846 \\
848 \\
850\end{array}$ & $\begin{array}{l}18.9 \\
18.9 \\
19.0 \\
19.0 \\
19.1\end{array}$ & $\begin{array}{l}612 \\
676 \\
739 \\
803 \\
865\end{array}$ & $\begin{array}{l}842 \\
844 \\
846 \\
848\end{array}$ & $\begin{array}{l}69.9 \\
70.0 \\
70.0 \\
70.1 \\
70.1\end{array}$ & & $\begin{array}{l}\varepsilon 42 \\
\varepsilon 44 \\
\varepsilon 46 \\
\varepsilon 48 \\
\varepsilon 50\end{array}$ & $\begin{array}{l}41.5 \\
41.6 \\
41.7 \\
41.8 \\
41.9\end{array}$ \\
\hline $\begin{array}{l}139904 \\
.140253 \\
140605 \\
140955 \\
141304\end{array}$ & $\begin{array}{l}852 \\
854 \\
856 \\
858 \\
860\end{array}$ & $\begin{array}{l}1.9 \\
1.9 \\
1.9 \\
1.9 \\
1.9\end{array}$ & $\begin{array}{l}1.28 \\
1.29 \\
1.29 \\
1.30 \\
1.30\end{array}$ & $\begin{array}{l}852 \\
854 \\
856 \\
858\end{array}$ & $\begin{array}{l}19.1 \\
19.2 \\
19.2 \\
19.3 \\
19.3\end{array}$ & $\begin{array}{l}929 \\
993 \\
055 \\
120 \\
184\end{array}$ & $\begin{array}{l}852 \\
854 \\
856 \\
858 \\
860\end{array}$ & $\begin{array}{l}70.1 \\
70.1 \\
70.1 \\
70.2 \\
70.3\end{array}$ & $\begin{array}{l}63 \\
66 \\
68 \\
70 \\
72\end{array}$ & $\begin{array}{l}\varepsilon 52 \\
\varepsilon 54 \\
\varepsilon 56 \\
\varepsilon 58 \\
\varepsilon 60\end{array}$ & $\begin{array}{l}42.0 \\
42.1 \\
42.2 \\
42.3 \\
42.4\end{array}$ \\
\hline $\begin{array}{l}141656 \\
142007 \\
142359 \\
142710 \\
143061\end{array}$ & $\begin{array}{l}862 \\
864 \\
866 \\
868\end{array}$ & $\begin{array}{l}1.9 \\
1.9 \\
1.9 \\
1.9 \\
1.9\end{array}$ & $\begin{array}{l}1.311735 \\
1.316237 \\
1.320745 \\
1.325260 \\
1.329788\end{array}$ & $\begin{array}{l}862 \\
864 \\
866 \\
868 \\
870\end{array}$ & $\begin{array}{l}19.3 \\
19.4 \\
19.4 \\
19.5 \\
19.5\end{array}$ & $\begin{array}{l}247 \\
311 \\
375 \\
439 \\
503\end{array}$ & $\begin{array}{l}862 \\
864 \\
866 \\
868 \\
870\end{array}$ & $\begin{array}{l}70.3 \\
70.3 \\
70.4 \\
70.4 \\
70.4\end{array}$ & $\begin{array}{l}974 \\
075 \\
177 \\
279 \\
380\end{array}$ & $\begin{array}{l}\varepsilon \varsigma 2 \\
\varepsilon \varsigma 4 \\
\varepsilon \varsigma 6 \\
\varepsilon \varsigma 8 \\
\varepsilon 70\end{array}$ & $\begin{array}{l}42.5 \\
42.6 \\
42.7 \\
42.8 \\
42.9\end{array}$ \\
\hline $\begin{array}{l}143413 \\
143763 \\
144116 \\
144467 \\
144819\end{array}$ & $\begin{array}{l}872 \\
874 \\
876 \\
878 \\
880\end{array}$ & $\begin{array}{l}1.9 \\
1.9 \\
1.9 \\
1.9 \\
2.0\end{array}$ & $\begin{array}{l}1.334321 \\
1.338864 \\
1.343416 \\
1.347976 \\
1.352546\end{array}$ & $\begin{array}{l}872 \\
874 \\
876 \\
878 \\
880\end{array}$ & $\begin{array}{l}19.6 \\
19.6 \\
19.7 \\
19.7 \\
19.7\end{array}$ & $\begin{array}{l}0.067567 \\
0.067632 \\
0.067696 \\
0.067760 \\
0.067825\end{array}$ & $\begin{array}{l}872 \\
874 \\
876 \\
878 \\
880\end{array}$ & $\begin{array}{l}70.5 \\
70.5 \\
70.5 \\
70.6 \\
70.6\end{array}$ & $\begin{array}{l}0.043482 \\
0.043584 \\
0.043687 \\
0.043789 \\
0.043890\end{array}$ & $\begin{array}{l}\varepsilon 72 \\
874 \\
876 \\
878 \\
890\end{array}$ & $\begin{array}{l}43.0 \\
43.1 \\
43.2 \\
43.3 \\
43.4\end{array}$ \\
\hline $\begin{array}{l}145172 \\
145523 \\
145875 \\
146228 \\
146580\end{array}$ & $\begin{array}{l}882 \\
884 \\
886 \\
888 \\
890\end{array}$ & $\begin{array}{l}2.0 \\
2.0 \\
2.0 \\
2.0 \\
2.0\end{array}$ & $\begin{array}{l}1.357126 \\
1.361713 \\
1.366308 \\
1.370912 \\
1.375528\end{array}$ & $\begin{array}{l}882 \\
884 \\
886 \\
888 \\
890\end{array}$ & $\begin{array}{l}19.8 \\
19.8 \\
19.9 \\
19.9 \\
20.0\end{array}$ & $\begin{array}{l}0.067889 \\
0.067954 \\
0.068019 \\
0.068084 \\
0.068149\end{array}$ & $\begin{array}{l}882 \\
884 \\
886 \\
888 \\
890\end{array}$ & $\begin{array}{l}70.6 \\
70.7 \\
70.7 \\
70.8 \\
70.8\end{array}$ & $\begin{array}{l}0.043992 \\
0.044095 \\
0.044196 \\
0.044298 \\
0.044399\end{array}$ & $\begin{array}{l}\varepsilon 82 \\
894 \\
\varepsilon 96 \\
898 \\
890\end{array}$ & $\begin{array}{l}43.5 \\
43.6 \\
43.7 \\
43.8 \\
43.9\end{array}$ \\
\hline $\begin{array}{l}146933 \\
147285 \\
147637 \\
147992 \\
148344\end{array}$ & $\begin{array}{l}892 \\
894 \\
896 \\
898 \\
900\end{array}$ & $\begin{array}{l}2.0 \\
2.0 \\
2.0 \\
2.0 \\
2.0\end{array}$ & $\begin{array}{l}1.380152 \\
1.384783 \\
1.389426 \\
1.394075 \\
1.398732\end{array}$ & $\begin{array}{l}892 \\
894 \\
896 \\
898 \\
900\end{array}$ & $\begin{array}{l}20.0 \\
20.1 \\
20.1 \\
20.2 \\
20.2\end{array}$ & $\begin{array}{l}0.068214 \\
0.068279 \\
0.068344 \\
0.068409 \\
0.068474\end{array}$ & $\begin{array}{l}892 \\
894 \\
896 \\
898 \\
900\end{array}$ & $\begin{array}{l}70.8 \\
70.9 \\
70.9 \\
70.9 \\
71.0\end{array}$ & $\begin{array}{l}0.044501 \\
0.044604 \\
0.044705 \\
0.044808 \\
0.044911\end{array}$ & $\begin{array}{l}892 \\
894 \\
896 \\
898 \\
900\end{array}$ & $\begin{array}{l}44.0 \\
44.1 \\
44.2 \\
44.3 \\
44.4\end{array}$ \\
\hline
\end{tabular}


Geologic age, in millions of years, calculated from the atomic ratios of indicated isotopes

\begin{tabular}{|c|c|c|c|c|c|c|c|c|c|c|c|}
\hline \multicolumn{3}{|c|}{$N_{206} / N_{238}$} & \multicolumn{3}{|c|}{$N_{207} / N_{235}$} & \multicolumn{3}{|c|}{$N_{207} / N_{206}$} & \multicolumn{3}{|c|}{$N_{208} / N_{232}$} \\
\hline \multirow[b]{2}{*}{ Ratio } & \multicolumn{2}{|c|}{ Age } & \multirow[b]{2}{*}{ Ratio } & \multicolumn{2}{|c|}{ Age } & \multirow[b]{2}{*}{ Ratio } & \multicolumn{2}{|c|}{ Age } & \multirow[b]{2}{*}{ Ratio } & \multicolumn{2}{|c|}{$\mathrm{Ag}$ ? } \\
\hline & \begin{tabular}{|c|}
$\begin{array}{c}\text { Number } \\
\text { of } \\
\text { years }\end{array}$ \\
\end{tabular} & $\begin{array}{c}\text { Error } \\
\pm\end{array}$ & & \begin{tabular}{|c|}
$\begin{array}{c}\text { Number } \\
\text { of } \\
\text { years }\end{array}$ \\
\end{tabular} & $\begin{array}{c}\text { Error } \\
\pm\end{array}$ & & \begin{tabular}{|c|}
$\begin{array}{c}\text { Number } \\
\text { of } \\
\text { years }\end{array}$ \\
\end{tabular} & $\begin{array}{c}\text { Error } \\
\pm\end{array}$ & & \begin{tabular}{|c|}
$\begin{array}{c}\text { Number } \\
\text { of } \\
\text { years }\end{array}$ \\
\end{tabular} & $\begin{array}{c}\text { Error } \\
\pm\end{array}$ \\
\hline $\begin{array}{l}0.148695 \\
0.149049 \\
0.149402 \\
0.149756 \\
0.150109\end{array}$ & $\begin{array}{l}902 \\
904 \\
906 \\
908 \\
910\end{array}$ & $\begin{array}{l}2.0 \\
2.0 \\
2.0 \\
2.0 \\
2.0\end{array}$ & $\begin{array}{l}1.403404 \\
1.408079 \\
1.412767 \\
1.417465 \\
1.422169\end{array}$ & $\begin{array}{l}902 \\
904 \\
906 \\
908 \\
910\end{array}$ & $\begin{array}{l}20.2 \\
20.3 \\
20.3 \\
20.4 \\
20.4\end{array}$ & $\begin{array}{l}0.068541 \\
0.068606 \\
0.068672 \\
0.068737 \\
0.068803\end{array}$ & $\begin{array}{l}902 \\
904 \\
906 \\
908 \\
910\end{array}$ & $\begin{array}{l}71.0 \\
71.1 \\
71.1 \\
71.1 \\
71.2\end{array}$ & $\begin{array}{l}0.045012 \\
0.045114 \\
0.045215 \\
0.045318 \\
0.045420\end{array}$ & $\begin{array}{l}902 \\
904 \\
906 \\
908 \\
910\end{array}$ & $\begin{array}{l}44.5 \\
44.6 \\
44.7 \\
44.8 \\
44.9\end{array}$ \\
\hline $\begin{array}{l}0.150463 \\
0.150817 \\
0.151170 \\
0.151526 \\
0.151879\end{array}$ & $\begin{array}{l}912 \\
914 \\
916 \\
918 \\
920\end{array}$ & $\begin{array}{l}2.0 \\
2.0 \\
2.0 \\
2.0 \\
2.0\end{array}$ & $\begin{array}{l}1.426881 \\
1.431607 \\
1.436339 \\
1.441078 \\
1.445831\end{array}$ & $\begin{array}{l}912 \\
914 \\
916 \\
918 \\
920\end{array}$ & $\begin{array}{l}20.5 \\
20.5 \\
20.6 \\
20.6 \\
20.6\end{array}$ & $\begin{array}{l}0.068869 \\
0.068934 \\
0.069001 \\
0.069066 \\
0.069133\end{array}$ & $\begin{array}{l}912 \\
914 \\
916 \\
918 \\
920\end{array}$ & $\begin{array}{l}71.2 \\
71.2 \\
71.3 \\
71.3 \\
71.3\end{array}$ & $\begin{array}{l}0.045521 \\
0.045624 \\
0.045726 \\
0.045827 \\
0.045931\end{array}$ & $\begin{array}{l}912 \\
914 \\
916 \\
918 \\
920\end{array}$ & $\begin{array}{l}45.0 \\
45.1 \\
45.2 \\
45.3 \\
45.4\end{array}$ \\
\hline $\begin{array}{l}0.152232 \\
0.152587 \\
0.152942 \\
0.153295 \\
0.153650\end{array}$ & $\begin{array}{l}922 \\
924 \\
926 \\
928 \\
930\end{array}$ & $\begin{array}{l}2.0 \\
2.0 \\
2.1 \\
2.1 \\
2.1\end{array}$ & $\begin{array}{l}1.450591 \\
1.455358 \\
1.460142 \\
1.464927 \\
1.469722\end{array}$ & $\begin{array}{l}922 \\
924 \\
926 \\
928 \\
930\end{array}$ & $\begin{array}{l}20.7 \\
20.7 \\
20.8 \\
20.8 \\
20.9\end{array}$ & $\begin{array}{l}0.069199 \\
0.069265 \\
0.069332 \\
0.069399 \\
0.069465\end{array}$ & $\begin{array}{l}922 \\
924 \\
926 \\
928 \\
930\end{array}$ & $\begin{array}{l}71.4 \\
71.4 \\
71.4 \\
71.5 \\
71.5\end{array}$ & $\begin{array}{l}0.046032 \\
0.046135 \\
0.046237 \\
0.046338 \\
0.046441\end{array}$ & $\begin{array}{l}922 \\
924 \\
926 \\
928 \\
930\end{array}$ & $\begin{array}{l}45.5 \\
45.5 \\
45.6 \\
45.7 \\
45.8\end{array}$ \\
\hline $\begin{array}{l}0.154004 \\
0.154360 \\
0.154715 \\
0.155069 \\
0.155425\end{array}$ & $\begin{array}{l}932 \\
934 \\
936 \\
938 \\
940\end{array}$ & $\begin{array}{l}2.1 \\
2.1 \\
2.1 \\
2.1 \\
2.1\end{array}$ & $\begin{array}{l}1.474533 \\
1.479348 \\
1.484172 \\
1.489006 \\
1.493850\end{array}$ & $\begin{array}{l}932 \\
934 \\
936 \\
938 \\
940\end{array}$ & $\begin{array}{l}20.9 \\
21.0 \\
21.0 \\
21.0 \\
21.1\end{array}$ & $\begin{array}{l}532 \\
598 \\
665 \\
732 \\
799\end{array}$ & $\begin{array}{l}932 \\
934 \\
936 \\
938 \\
940\end{array}$ & $\begin{array}{l}71.6 \\
71.6 \\
71.6 \\
71.6 \\
71.7\end{array}$ & $\begin{array}{l}0.046543 \\
0.046645 \\
0.046747 \\
0.046849 \\
0.046952\end{array}$ & $\begin{array}{l}932 \\
934 \\
936 \\
938 \\
940\end{array}$ & $\begin{array}{l}45.9 \\
46.0 \\
46.1 \\
46.2 \\
46.3\end{array}$ \\
\hline $\begin{array}{l}0.155780 \\
0.156135 \\
0.156491 \\
0.156846 \\
0.157202\end{array}$ & $\begin{array}{l}942 \\
944 \\
946 \\
948 \\
950\end{array}$ & $\begin{array}{l}2.1 \\
2.1 \\
2.1 \\
2.1 \\
2.1\end{array}$ & $\begin{array}{l}1.498706 \\
1.503566 \\
1.508441 \\
1.513320 \\
1.518213\end{array}$ & $\begin{array}{l}942 \\
944 \\
946 \\
948 \\
950\end{array}$ & $\begin{array}{l}21.1 \\
21.2 \\
21.2 \\
21.3 \\
21.3\end{array}$ & $\begin{array}{l}0.069866 \\
0.069933 \\
0.070001 \\
0.070068 \\
0.070135\end{array}$ & $\begin{array}{l}942 \\
944 \\
946 \\
948 \\
950\end{array}$ & $\begin{array}{l}71.7 \\
71.7 \\
71.8 \\
71.8 \\
71.9\end{array}$ & $\begin{array}{l}0.047055 \\
0.047156 \\
0.047259 \\
0.047361 \\
0.047463\end{array}$ & $\begin{array}{l}942 \\
944 \\
946 \\
948 \\
950\end{array}$ & $\begin{array}{l}46.4 \\
46.5 \\
46.6 \\
46.7 \\
46.8\end{array}$ \\
\hline $\begin{array}{l}0.157557 \\
0.157913 \\
0.158270 \\
0.158625 \\
0.158982\end{array}$ & $\begin{array}{l}952 \\
954 \\
956 \\
958 \\
960\end{array}$ & $\begin{array}{l}2.1 \\
2.1 \\
2.1 \\
2.1 \\
2.1\end{array}$ & $\begin{array}{l}1.523116 \\
1.528023 \\
1.532944 \\
1.537874 \\
1.542813\end{array}$ & $\begin{array}{l}952 \\
954 \\
956 \\
958 \\
960\end{array}$ & $\begin{array}{l}21.4 \\
21.4 \\
21.5 \\
21.5 \\
21.5\end{array}$ & $\begin{array}{l}0.070203 \\
0.070271 \\
0.070338 \\
0.070406 \\
0.070474\end{array}$ & $\begin{array}{l}952 \\
954 \\
956 \\
958 \\
960\end{array}$ & $\begin{array}{l}71.9 \\
72.0 \\
72.0 \\
72.0 \\
72.1\end{array}$ & $\begin{array}{l}0.047565 \\
0.047667 \\
0.047769 \\
0.047873 \\
0.047974\end{array}$ & $\begin{array}{l}952 \\
954 \\
956 \\
958 \\
960\end{array}$ & $\begin{array}{l}46.9 \\
47.0 \\
47.1 \\
47.2 \\
47.3\end{array}$ \\
\hline $\begin{array}{l}0.159337 \\
0.159695 \\
0.160052 \\
0.160407 \\
0.160765\end{array}$ & $\begin{array}{l}962 \\
964 \\
966 \\
968 \\
970\end{array}$ & $\begin{array}{l}2.1 \\
2.1 \\
2.1 \\
2.1 \\
2.1\end{array}$ & $\begin{array}{l}1.547763 \\
1.552720 \\
1.557689 \\
1.562665 \\
1.567654\end{array}$ & $\begin{array}{l}962 \\
964 \\
966 \\
968 \\
970\end{array}$ & $\begin{array}{l}21.6 \\
21.6 \\
21.7 \\
21.7 \\
21.8\end{array}$ & $\begin{array}{l}0.070542 \\
0.070610 \\
0.070678 \\
0.070747 \\
0.070814\end{array}$ & $\begin{array}{l}962 \\
964 \\
966 \\
968 \\
970\end{array}$ & $\begin{array}{l}72.1 \\
72.1 \\
72.2 \\
72.2 \\
72.2\end{array}$ & $\begin{array}{l}0.048077 \\
0.048180 \\
0.048281 \\
0.048384 \\
0.048485\end{array}$ & $\begin{array}{l}962 \\
964 \\
966 \\
968 \\
970\end{array}$ & $\begin{array}{l}47.4 \\
47.5 \\
47.6 \\
47.7 \\
47.8\end{array}$ \\
\hline $\begin{array}{l}0.161121 \\
0.161478 \\
0.161836 \\
0.162192 \\
0.162549\end{array}$ & $\begin{array}{l}972 \\
974 \\
976 \\
978 \\
980\end{array}$ & $\begin{array}{l}2.2 \\
2.2 \\
2.2 \\
2.2 \\
2.2\end{array}$ & $\begin{array}{l}1.572651 \\
1.577656 \\
1.582677 \\
1.587702 \\
1.592736\end{array}$ & $\begin{array}{l}972 \\
974 \\
976 \\
978 \\
980\end{array}$ & $\begin{array}{l}21.8 \\
21.9 \\
21.9 \\
21.9 \\
22.0\end{array}$ & $\begin{array}{l}0.070883 \\
0.070952 \\
0.071020 \\
0.071089 \\
0.071158\end{array}$ & $\begin{array}{l}972 \\
974 \\
976 \\
978 \\
980\end{array}$ & $\begin{array}{l}72.3 \\
72.3 \\
72.3 \\
72.4 \\
72.4\end{array}$ & $\begin{array}{l}0.048588 \\
0.048691 \\
0.048793 \\
0.048896 \\
0.048999\end{array}$ & $\begin{array}{l}972 \\
974 \\
976 \\
978 \\
980\end{array}$ & $\begin{array}{l}47.9 \\
48.0 \\
48.1 \\
48.2 \\
48.3\end{array}$ \\
\hline $\begin{array}{l}0.162907 \\
0.163264 \\
0.163622 \\
0.163980 \\
0.164338\end{array}$ & $\begin{array}{l}982 \\
984 \\
986 \\
988 \\
990\end{array}$ & $\begin{array}{l}2.2 \\
2.2 \\
2.2 \\
2.2 \\
2.2\end{array}$ & $\begin{array}{l}1.597785 \\
1.602840 \\
1.607905 \\
1.612980 \\
1.618067\end{array}$ & $\begin{array}{l}982 \\
984 \\
986 \\
988 \\
990\end{array}$ & $\begin{array}{l}22.0 \\
22.1 \\
22.1 \\
22.2 \\
22.2\end{array}$ & $\begin{array}{l}0.071227 \\
0.071296 \\
0.071364 \\
0.071433 \\
0.071503\end{array}$ & $\begin{array}{l}982 \\
984 \\
986 \\
988 \\
990\end{array}$ & $\begin{array}{l}72.5 \\
72.5 \\
72.5 \\
72.6 \\
72.6\end{array}$ & $\begin{array}{l}0.049100 \\
0.049203 \\
0.049305 \\
0.049407 \\
0.049510\end{array}$ & $\begin{array}{l}982 \\
984 \\
986 \\
988 \\
990\end{array}$ & $\begin{array}{l}48.4 \\
48.5 \\
48.6 \\
48.7 \\
48.8\end{array}$ \\
\hline $\begin{array}{l}0.164696 \\
0.165054 \\
0.165412 \\
0.165771 \\
0.166129\end{array}$ & $\begin{array}{r}992 \\
994 \\
996 \\
998 \\
1000\end{array}$ & $\begin{array}{l}2.2 \\
2.2 \\
2.2 \\
2.2 \\
2.2\end{array}$ & $\begin{array}{l}1.623161 \\
1.628263 \\
1.633384 \\
1.638507 \\
1.643640\end{array}$ & $\begin{array}{r}992 \\
994 \\
996 \\
998 \\
1000\end{array}$ & $\begin{array}{l}22.3 \\
22.3 \\
22.4 \\
22.4 \\
22.4\end{array}$ & $\begin{array}{l}0.071572 \\
0.071641 \\
0.071711 \\
0.071780 \\
0.071850\end{array}$ & $\begin{array}{r}992 \\
994 \\
996 \\
998 \\
1000\end{array}$ & $\begin{array}{l}72.6 \\
72.7 \\
72.7 \\
72.7 \\
72.8\end{array}$ & $\begin{array}{l}0.049612 \\
0.049716 \\
0.049818 \\
0.049920 \\
0.050023\end{array}$ & $\begin{array}{r}992 \\
994 \\
996 \\
998 \\
1000\end{array}$ & $\begin{array}{l}48.9 \\
49.0 \\
49.1 \\
49.2 \\
49.3\end{array}$ \\
\hline
\end{tabular}


Geologic age, in millions of years, calculated from the atomic ratios of indicated isotopes

\begin{tabular}{|c|c|c|c|c|c|c|c|c|c|c|c|}
\hline \multicolumn{3}{|c|}{$N_{206} / N_{238}$} & \multicolumn{3}{|c|}{$N_{207} / N_{235}$} & \multicolumn{3}{|c|}{$N_{207} / N_{206}$} & \multicolumn{3}{|c|}{$N_{208} / N_{232}$} \\
\hline \multirow[b]{2}{*}{ Ratio } & \multicolumn{2}{|c|}{ Age } & \multirow[b]{2}{*}{ Ratio } & \multicolumn{2}{|c|}{ Age } & \multirow[b]{2}{*}{ Ratio } & \multicolumn{2}{|c|}{ Age } & \multirow[b]{2}{*}{ Ratio } & \multicolumn{2}{|c|}{ Age } \\
\hline & \begin{tabular}{|c|}
$\begin{array}{c}\text { Number } \\
\text { of } \\
\text { years }\end{array}$ \\
\end{tabular} & $\begin{array}{c}\text { Error } \\
\pm\end{array}$ & & \begin{tabular}{|c|} 
Number \\
of \\
years
\end{tabular} & $\begin{array}{c}\text { Error } \\
\pm\end{array}$ & & \begin{tabular}{|c|}
$\begin{array}{c}\text { Number } \\
\text { of } \\
\text { years }\end{array}$ \\
\end{tabular} & $\begin{array}{c}\text { Error } \\
\pm\end{array}$ & & \begin{tabular}{|c|}
$\begin{array}{c}\text { Number } \\
\text { of } \\
\text { years }\end{array}$ \\
\end{tabular} & $\begin{array}{c}\text { Error } \\
\pm\end{array}$ \\
\hline $\begin{array}{l}0.166487 \\
0.166846 \\
0.167203 \\
0.167563 \\
0.167922\end{array}$ & $\begin{array}{l}1002 \\
1004 \\
1006 \\
1008 \\
1010\end{array}$ & $\begin{array}{l}2.2 \\
2.2 \\
2.2 \\
2.2 \\
2.2\end{array}$ & $\begin{array}{l}1.65 \\
1.65 \\
1.66 \\
1.66\end{array}$ & $\begin{array}{l}1002 \\
1004 \\
1006 \\
1008 \\
1010\end{array}$ & $\begin{array}{l}22.5 \\
22.5 \\
22.6 \\
22.6 \\
22.7\end{array}$ & $\begin{array}{l}920 \\
989 \\
060 \\
129 \\
199\end{array}$ & $\begin{array}{l}1002 \\
1004 \\
1006 \\
1008 \\
1010\end{array}$ & $\begin{array}{l}72 \\
72 \\
72 \\
73\end{array}$ & $\begin{array}{l}126 \\
227 \\
330 \\
432 \\
535\end{array}$ & $\begin{array}{l}1002 \\
1004 \\
1006 \\
1008 \\
1010\end{array}$ & $\begin{array}{l}49.5 \\
49.6 \\
49.7 \\
49.8\end{array}$ \\
\hline $\begin{array}{l}0.168282 \\
0.168640 \\
0.168999 \\
0.169359 \\
0.169719\end{array}$ & $\begin{array}{l}1012 \\
1014 \\
1016 \\
1018 \\
1020\end{array}$ & $\begin{array}{l}2.2 \\
2.2 \\
2.3 \\
2.3 \\
2.3\end{array}$ & $\begin{array}{l}1.6 \\
1.6 \\
1.68 \\
1.6 \\
1.6\end{array}$ & $\begin{array}{l}1012 \\
1014 \\
1016 \\
1018 \\
1020\end{array}$ & $\begin{array}{l}22.7 \\
22.8 \\
22.8 \\
22.8 \\
22.9\end{array}$ & $\begin{array}{l}269 \\
340 \\
410 \\
880 \\
451\end{array}$ & $\begin{array}{l}1012 \\
1014 \\
1016 \\
1018 \\
1020\end{array}$ & $\begin{array}{l}73.1 \\
73.1 \\
73.1 \\
73.2\end{array}$ & $\begin{array}{l}39 \\
40 \\
43 \\
46 \\
48\end{array}$ & $\begin{array}{l}1012 \\
1014 \\
1016 \\
1018 \\
1020\end{array}$ & $\begin{array}{l}50.0 \\
50.1 \\
50.2 \\
50.3\end{array}$ \\
\hline $\begin{array}{l}0.170078 \\
0.170438 \\
0.170797 \\
0.171158 \\
0.171518\end{array}$ & $\begin{array}{l}1022 \\
1024 \\
1026 \\
1028 \\
1030\end{array}$ & $\begin{array}{l}2.3 \\
2.3 \\
2.3 \\
2.3 \\
2.3\end{array}$ & $\begin{array}{l}1.70 \\
1.70 \\
1.71 \\
1.71 \\
1.72\end{array}$ & $\begin{array}{l}1022 \\
1024 \\
1026 \\
1028 \\
1030\end{array}$ & $\begin{array}{l}22 \\
23 \\
23 \\
23 \\
23\end{array}$ & $\begin{array}{l}622 \\
692 \\
763 \\
834 \\
905\end{array}$ & $\begin{array}{l}1022 \\
1024 \\
1026 \\
1028 \\
1030\end{array}$ & $\begin{array}{l}73 \\
73 \\
73 \\
73\end{array}$ & & $\begin{array}{l}1022 \\
1024 \\
1026 \\
1028 \\
1030\end{array}$ & $\begin{array}{l}5 \\
5 \\
5 \\
5\end{array}$ \\
\hline $\begin{array}{l}0.171877 \\
0.172238 \\
0.172598 \\
0.172959 \\
0.173320\end{array}$ & $\begin{array}{l}1032 \\
1034 \\
1036 \\
1038 \\
1040\end{array}$ & $\begin{array}{l}2.3 \\
2.3 \\
2.3 \\
2.3 \\
2.3\end{array}$ & $\begin{array}{l}1.72 \\
1.73 \\
1.73 \\
1.74 \\
1.74\end{array}$ & $\begin{array}{l}1032 \\
1034 \\
1036 \\
1038 \\
1040\end{array}$ & $\begin{array}{l}23 \\
23 \\
23 \\
23 \\
23\end{array}$ & $\begin{array}{l}976 \\
047 \\
119 \\
190 \\
261\end{array}$ & $\begin{array}{l}1032 \\
1034 \\
1036 \\
1038 \\
1040\end{array}$ & $\begin{array}{l}73 \\
73 \\
73 \\
73\end{array}$ & & $\begin{array}{l}10 \\
10 \\
10 \\
10 \\
10\end{array}$ & $\begin{array}{l}5] \\
5] \\
5] \\
5]\end{array}$ \\
\hline $\begin{array}{l}0.173680 \\
0.174041 \\
0.174402 \\
0.174764 \\
0.175125\end{array}$ & $\begin{array}{l}1042 \\
1044 \\
1046 \\
1048 \\
1050\end{array}$ & $\begin{array}{l}2.3 \\
2.3 \\
2.3 \\
2.3 \\
2.3\end{array}$ & $\begin{array}{l}1.75 \\
1.75 \\
1.76 \\
1.76 \\
1.77\end{array}$ & $\begin{array}{l}1042 \\
1044 \\
1046 \\
1048 \\
1050\end{array}$ & $\begin{array}{l}23 \\
23 \\
23 \\
23 \\
23\end{array}$ & $\begin{array}{l}333 \\
404 \\
476 \\
547 \\
619\end{array}$ & $\begin{array}{l}1042 \\
1044 \\
1046 \\
1048 \\
1050\end{array}$ & $\begin{array}{l}73 \\
73 \\
73 \\
73 \\
73\end{array}$ & $\begin{array}{l}77 \\
81 \\
83 \\
86 \\
89\end{array}$ & $\begin{array}{l}10 \\
10 \\
10 \\
10 \\
10\end{array}$ & $\begin{array}{l}5] \\
5] \\
5] \\
5]\end{array}$ \\
\hline $\begin{array}{l}0.175485 \\
0.175847 \\
0.176208 \\
0.176570 \\
0.176932\end{array}$ & $\begin{array}{l}1052 \\
1054 \\
1056 \\
1058 \\
1060\end{array}$ & $\begin{array}{l}2.3 \\
2.3 \\
2.3 \\
2.3 \\
2.4\end{array}$ & $\begin{array}{l}1.78 \\
1.78 \\
1.79 \\
1.79 \\
1.80\end{array}$ & $\begin{array}{l}1052 \\
1054 \\
1056 \\
1058 \\
1060\end{array}$ & $\begin{array}{l}23 \\
23 \\
23 \\
23 \\
23\end{array}$ & $\left\{\begin{array}{l}92 \\
64 \\
336 \\
08 \\
80\end{array}\right.$ & $\begin{array}{l}1052 \\
1054 \\
1056 \\
1058 \\
1060\end{array}$ & $\begin{array}{l}73 \\
73 \\
73 \\
73 \\
73\end{array}$ & & $\begin{array}{l}10 \\
10 \\
10 \\
10 \\
10\end{array}$ & $\begin{array}{l}52.0 \\
52.1 \\
52.2 \\
52.3\end{array}$ \\
\hline $\begin{array}{l}0.177293 \\
0.177655 \\
0.178016 \\
0.178380 \\
0.178742\end{array}$ & $\begin{array}{l}1062 \\
1064 \\
1066 \\
1068 \\
1070\end{array}$ & $\begin{array}{l}2.4 \\
2.4 \\
2.4 \\
2.4 \\
2.4\end{array}$ & $\begin{array}{l}1.80 \\
1.81 \\
1.81 \\
1.82 \\
1.82\end{array}$ & $\begin{array}{l}1062 \\
1064 \\
1066 \\
1068 \\
1070\end{array}$ & $\begin{array}{l}23 \\
23 \\
23 \\
24 \\
24\end{array}$ & $\begin{array}{l}053 \\
125 \\
198 \\
270 \\
343\end{array}$ & $\begin{array}{l}1062 \\
1064 \\
1066 \\
1068 \\
1070\end{array}$ & $\begin{array}{l}73 \\
74 \\
74\end{array}$ & $\begin{array}{l}205 \\
308 \\
411 \\
513 \\
616\end{array}$ & $\begin{array}{l}1062 \\
1064 \\
1066 \\
1068 \\
1070\end{array}$ & $\begin{array}{l}52.4 \\
52.5 \\
52.5 \\
52.6 \\
52.7\end{array}$ \\
\hline $\begin{array}{l}0.179104 \\
0.179466 \\
0.179830 \\
0.180192 \\
0.180556\end{array}$ & $\begin{array}{l}1072 \\
1074 \\
1076 \\
1078 \\
1080\end{array}$ & $\begin{array}{l}2.4 \\
2.4 \\
2.4 \\
2.4 \\
2.4\end{array}$ & $\begin{array}{l}1.8 \\
1.8 \\
1.8 \\
1.8 \\
1.8\end{array}$ & $\begin{array}{l}1072 \\
1074 \\
1076 \\
1078 \\
1080\end{array}$ & $\begin{array}{l}24.1 \\
24.1 \\
24.1 \\
24.2 \\
24.2\end{array}$ & $\begin{array}{l}4416 \\
4489 \\
4562 \\
4636 \\
4708\end{array}$ & $\begin{array}{l}1072 \\
1074 \\
1076 \\
1078 \\
1080\end{array}$ & $\begin{array}{l}74 \\
74 \\
74 \\
74 \\
74\end{array}$ & $\begin{array}{l}19 \\
23 \\
26 \\
28 \\
31\end{array}$ & & $\begin{array}{l}52.8 \\
52.9 \\
53.0 \\
53.1 \\
53.2\end{array}$ \\
\hline $\begin{array}{l}0.180918 \\
0.181281 \\
0.181645 \\
0.182008 \\
0.182372\end{array}$ & $\begin{array}{l}1082 \\
1084 \\
1086 \\
1088 \\
1090\end{array}$ & $\begin{array}{l}2.4 \\
2.4 \\
2.4 \\
2.4 \\
2.4\end{array}$ & $\begin{array}{l}1.8630_{12} \\
1.868587 \\
1.874168 \\
1.879760 \\
1.885368\end{array}$ & $\begin{array}{l}1082 \\
1084 \\
1086 \\
1088 \\
1090\end{array}$ & $\begin{array}{l}24.3 \\
24.3 \\
24.4 \\
24.4 \\
24.5\end{array}$ & $\begin{array}{l}0.074782 \\
0.074856 \\
0.074929 \\
0.075002 \\
0.075076\end{array}$ & $\begin{array}{l}1082 \\
1084 \\
1086 \\
1088 \\
1090\end{array}$ & $\begin{array}{l}74.3 \\
74.4 \\
74.4 \\
74.4 \\
74.4\end{array}$ & $\begin{array}{l}0,054234 \\
0.054336 \\
0.054440 \\
0.054542 \\
0.054646\end{array}$ & $\begin{array}{l}1082 \\
1084 \\
1086 \\
1088 \\
1090\end{array}$ & $\begin{array}{l}53.3 \\
53.4 \\
53.5 \\
53.6 \\
53.7\end{array}$ \\
\hline $\begin{array}{l}0.182735 \\
0.183099 \\
0.183463 \\
0.183825 \\
0.184190\end{array}$ & $\begin{array}{l}1092 \\
1094 \\
1096 \\
1098 \\
1100\end{array}$ & $\begin{array}{l}2.4 \\
2.4 \\
2.4 \\
2.4 \\
2.4\end{array}$ & $\begin{array}{l}1.890980 \\
1.896606 \\
1902248 \\
1.907894 \\
1.913552\end{array}$ & $\begin{array}{l}1092 \\
1094 \\
1096 \\
1098 \\
1100\end{array}$ & $\begin{array}{l}24.5 \\
24.5 \\
24.6 \\
24.6 \\
24.7\end{array}$ & $\begin{array}{l}0.075150 \\
0.075224 \\
0.075298 \\
0.075372 \\
0.075446\end{array}$ & $\begin{array}{l}1092 \\
1094 \\
1096 \\
1098 \\
1100\end{array}$ & $\begin{array}{l}74.5 \\
74.5 \\
74.6 \\
74.6 \\
74.6\end{array}$ & $\begin{array}{l}0.054749 \\
0.054851 \\
0.054955 \\
0.055058 \\
0.055160\end{array}$ & $\begin{array}{l}1092 \\
1094 \\
1096 \\
1098 \\
1100\end{array}$ & $\begin{array}{l}53.8 \\
53.9 \\
54.0 \\
54.1 \\
54.2\end{array}$ \\
\hline
\end{tabular}


Geologic age, in millions of years, calculated from the atomic ratios of indicated isotopes

\begin{tabular}{|c|c|c|c|c|c|c|c|c|c|c|c|}
\hline \multicolumn{3}{|c|}{$N_{206} / N_{238}$} & \multicolumn{3}{|c|}{$N_{20 z / N_{235}}$} & \multicolumn{3}{|c|}{$N_{207} / N_{206}$} & \multicolumn{3}{|c|}{$N_{208} / N_{232}$} \\
\hline \multirow[b]{2}{*}{ Ratio } & \multicolumn{2}{|c|}{ Age } & \multirow[b]{2}{*}{ Ratio } & \multicolumn{2}{|c|}{ Age } & \multirow[b]{2}{*}{ Ratio } & \multicolumn{2}{|c|}{ Age } & \multirow[b]{2}{*}{ Ratio } & \multicolumn{2}{|c|}{ Age } \\
\hline & \begin{tabular}{|c} 
Number \\
of \\
years
\end{tabular} & $\begin{array}{c}\text { Error } \\
\pm\end{array}$ & & $\begin{array}{c}\text { Number } \\
\text { of } \\
\text { years }\end{array}$ & $\begin{array}{c}\text { Error } \\
\pm\end{array}$ & & \begin{tabular}{|c|}
$\begin{array}{c}\text { Number } \\
\text { of } \\
\text { years }\end{array}$ \\
\end{tabular} & $\begin{array}{c}\text { Error } \\
\pm \\
\end{array}$ & & \begin{tabular}{|c|}
$\begin{array}{c}\text { Number } \\
\text { of } \\
\text { years }\end{array}$ \\
\end{tabular} & $\begin{array}{c}\text { Error } \\
\pm\end{array}$ \\
\hline $\begin{array}{l}0.184554 \\
0.184918 \\
0.185283 \\
0.185648 \\
0.1860_{11}\end{array}$ & $\begin{array}{l}1102 \\
1104 \\
1106 \\
1108 \\
1110\end{array}$ & $\begin{array}{l}2.4 \\
2.4 \\
2.5 \\
2.5 \\
2.5\end{array}$ & $\begin{array}{l}1.919225 \\
1.924905 \\
1.930598 \\
1.936302 \\
1.942016\end{array}$ & $\begin{array}{l}1102 \\
1104 \\
1106 \\
1108 \\
1110\end{array}$ & $\begin{array}{l}24.7 \\
24.8 \\
24.8 \\
24.9 \\
24.9\end{array}$ & $\begin{array}{l}0.075521 \\
0.075595 \\
0.075669 \\
0.075744 \\
0.075819\end{array}$ & $\begin{array}{l}1102 \\
1104 \\
1106 \\
1108 \\
1110\end{array}$ & $\begin{array}{l}74.7 \\
74.7 \\
74.7 \\
74.8 \\
74.8\end{array}$ & $\begin{array}{l}0.055263 \\
0.055367 \\
0.055470 \\
0.055573 \\
0.055675\end{array}$ & $\begin{array}{l}1102 \\
1104 \\
1106 \\
1108 \\
111 C\end{array}$ & $\begin{array}{l}54.3 \\
54.4 \\
54.5 \\
54.6 \\
54.7\end{array}$ \\
\hline $\begin{array}{l}0.186376 \\
0.186740 \\
0.187106 \\
0.187471 \\
0.187836\end{array}$ & $\begin{array}{l}1112 \\
1114 \\
1116 \\
1118 \\
1120\end{array}$ & $\begin{array}{l}2.5 \\
2.5 \\
2.5 \\
2.5 \\
2.5\end{array}$ & $\begin{array}{l}1.947741 \\
1.953480 \\
1.959227 \\
1.964985 \\
1.970758\end{array}$ & $\begin{array}{l}1112 \\
1114 \\
1116 \\
1118 \\
1120\end{array}$ & $\begin{array}{l}25.0 \\
25.0 \\
25.0 \\
25.1 \\
25.1\end{array}$ & $\begin{array}{l}0.075893 \\
0.075969 \\
0.076043 \\
0.076118 \\
0.076193\end{array}$ & $\begin{array}{l}1112 \\
1114 \\
1116 \\
1118 \\
1120\end{array}$ & $\begin{array}{l}74.8 \\
74.9 \\
74.9 \\
74.9 \\
75.0\end{array}$ & $\begin{array}{l}779 \\
882 \\
984 \\
089 \\
191\end{array}$ & $\begin{array}{l}1112 \\
1114 \\
1116 \\
1118 \\
112 \mathrm{C}\end{array}$ & $\begin{array}{l}54.8 \\
54.9 \\
55.0 \\
55.1 \\
55.2\end{array}$ \\
\hline $\begin{array}{l}0.188201 \\
0.188567 \\
0.188931 \\
0.189297 \\
0.189661\end{array}$ & $\begin{array}{l}1122 \\
1124 \\
1126 \\
1128 \\
1130\end{array}$ & $\begin{array}{l}2.5 \\
2.5 \\
2.5 \\
2.5 \\
2.5\end{array}$ & $\begin{array}{l}1.976537 \\
1.982333 \\
1.988138 \\
1.993953 \\
1.999777\end{array}$ & $\begin{array}{l}1122 \\
1124 \\
1126 \\
1128 \\
1130\end{array}$ & $\begin{array}{l}25.2 \\
25.2 \\
25.3 \\
25.3 \\
25.4\end{array}$ & $\begin{array}{l}0.076269 \\
0.076344 \\
0.076420 \\
0.076495 \\
0.076571\end{array}$ & $\begin{array}{l}1122 \\
1124 \\
1126 \\
1128 \\
1130\end{array}$ & $\begin{array}{l}75.0 \\
75.1 \\
75.1 \\
75.2 \\
75.2\end{array}$ & $\begin{array}{l}294 \\
398 \\
500 \\
603 \\
707\end{array}$ & $\begin{array}{l}1122 \\
1124 \\
1126 \\
1128 \\
1130\end{array}$ & $\begin{array}{l}55.3 \\
55.4 \\
55.5 \\
55.6 \\
55.7\end{array}$ \\
\hline $\begin{array}{l}0.190029 \\
0.190394 \\
0.190759 \\
0.191126 \\
0.191492\end{array}$ & $\begin{array}{l}1132 \\
1134 \\
1136 \\
1138 \\
1140\end{array}$ & $\begin{array}{l}2.5 \\
2.5 \\
2.5 \\
2.5 \\
2.5\end{array}$ & $\begin{array}{l}2.005615 \\
2.011466 \\
2.017325 \\
2.023197 \\
2.029084\end{array}$ & $\begin{array}{l}1132 \\
1134 \\
1136 \\
1138 \\
1140\end{array}$ & $\begin{array}{l}25.4 \\
25.4 \\
25.5 \\
25.5 \\
25.6\end{array}$ & $\begin{array}{l}0.076646 \\
0.076722 \\
0.076799 \\
0.076874 \\
0.076951\end{array}$ & $\begin{array}{l}1132 \\
1134 \\
1136 \\
1138 \\
1140\end{array}$ & $\begin{array}{l}75.2 \\
75.2 \\
75.3 \\
75.3 \\
75.4\end{array}$ & $\begin{array}{l}810 \\
914 \\
016 \\
119 \\
223\end{array}$ & $\begin{array}{l}1132 \\
1134 \\
1136 \\
1138 \\
114 C\end{array}$ & $\begin{array}{l}55.8 \\
55.9 \\
56.0 \\
56.1 \\
56.2\end{array}$ \\
\hline $\begin{array}{l}0.191859 \\
0.192224 \\
0.192591 \\
0.192959 \\
0.193325\end{array}$ & $\begin{array}{l}1142 \\
1144 \\
1146 \\
1148 \\
1150\end{array}$ & $\begin{array}{l}2.5 \\
2.5 \\
2.5 \\
2.5 \\
2.5\end{array}$ & $\begin{array}{l}2.034978 \\
2.040884 \\
2.046803 \\
2.052732 \\
2.058672\end{array}$ & $\begin{array}{l}1142 \\
1144 \\
1146 \\
1148 \\
1150\end{array}$ & $\begin{array}{l}25.6 \\
25.7 \\
25.7 \\
25.8 \\
25.8\end{array}$ & $\begin{array}{l}0.077027 \\
0.077103 \\
0.077180 \\
0.077256 \\
0.077333\end{array}$ & $\begin{array}{l}1142 \\
1144 \\
1146 \\
1148 \\
1150\end{array}$ & $\begin{array}{l}75.4 \\
75.4 \\
75.5 \\
75.5 \\
75.6\end{array}$ & $\begin{array}{l}325 \\
430 \\
533 \\
636 \\
739\end{array}$ & $\begin{array}{l}1142 \\
1144 \\
1146 \\
1148 \\
115 C\end{array}$ & $\begin{array}{l}56.3 \\
56.4 \\
56.5 \\
56.6 \\
56.7\end{array}$ \\
\hline $\begin{array}{l}0.193690 \\
0.194059 \\
0.194425 \\
0.194793 \\
0.195161\end{array}$ & $\begin{array}{l}1152 \\
1154 \\
1156 \\
1158 \\
1160\end{array}$ & $\begin{array}{l}2.6 \\
2.6 \\
2.6 \\
2.6 \\
2.6\end{array}$ & $\begin{array}{l}2.064629 \\
2.070591 \\
2.076567 \\
2.082554 \\
2.088556\end{array}$ & $\begin{array}{l}1152 \\
1154 \\
1156 \\
1158 \\
1160\end{array}$ & $\begin{array}{l}25.9 \\
25.9 \\
25.9 \\
26.0 \\
26.0\end{array}$ & $\begin{array}{l}0.077410 \\
0.077486 \\
0.077563 \\
0.077640 \\
0.077717\end{array}$ & $\begin{array}{l}1152 \\
1154 \\
1156 \\
1158 \\
1160\end{array}$ & $\begin{array}{l}75.6 \\
75.6 \\
75.7 \\
75.7 \\
75.7\end{array}$ & $\begin{array}{l}7843 \\
7945 \\
3049 \\
3152 \\
3256\end{array}$ & $\begin{array}{l}1152 \\
1154 \\
1156 \\
115 \varepsilon \\
116 c\end{array}$ & $\begin{array}{l}56.8 \\
56.9 \\
57.0 \\
57.1 \\
57.2\end{array}$ \\
\hline $\begin{array}{l}0.195528 \\
0.195896 \\
0.196263 \\
0.196632 \\
0.196999\end{array}$ & $\begin{array}{l}1162 \\
1164 \\
1166 \\
1168 \\
1170\end{array}$ & $\begin{array}{l}2.6 \\
2.6 \\
2.6 \\
2.6 \\
2.6\end{array}$ & $\begin{array}{l}2.094564 \\
2.100590 \\
2.106624 \\
2.112667 \\
2.118727\end{array}$ & $\begin{array}{l}1162 \\
1164 \\
1166 \\
1168 \\
1170\end{array}$ & $\begin{array}{l}26.1 \\
26.1 \\
26.2 \\
26.2 \\
26.3\end{array}$ & $\begin{array}{l}0.077794 \\
0.077872 \\
0.077949 \\
0.078026 \\
0.078104\end{array}$ & $\begin{array}{l}1162 \\
1164 \\
1166 \\
1168 \\
1170\end{array}$ & $\begin{array}{l}75.8 \\
75.8 \\
75.9 \\
75.9 \\
75.9\end{array}$ & $\begin{array}{l}359 \\
462 \\
565 \\
669 \\
772\end{array}$ & $\begin{array}{l}1162 \\
1164 \\
1166 \\
1168 \\
117 c\end{array}$ & $\begin{array}{l}57.3 \\
57.4 \\
57.5 \\
57.6 \\
57.7\end{array}$ \\
\hline $\begin{array}{l}0.197366 \\
0.197736 \\
0.198103 \\
0.198471 \\
0.198840\end{array}$ & $\begin{array}{l}1172 \\
1174 \\
1176 \\
1178 \\
1180\end{array}$ & $\begin{array}{l}2.6 \\
2.6 \\
2.6 \\
2.6 \\
2.6\end{array}$ & $\begin{array}{l}2.124797 \\
2.130878 \\
2.136974 \\
2.143077 \\
2.149193\end{array}$ & $\begin{array}{l}1172 \\
1174 \\
1176 \\
1178 \\
1180\end{array}$ & $\begin{array}{l}26.3 \\
26.3 \\
26.4 \\
26.4 \\
26.5\end{array}$ & $\begin{array}{l}0.078182 \\
0.078259 \\
0.078338 \\
0.078416 \\
0.078494\end{array}$ & $\begin{array}{l}1172 \\
1174 \\
1176 \\
1178 \\
1180\end{array}$ & $\begin{array}{l}76.0 \\
76.0 \\
76.1 \\
76.1 \\
76.1\end{array}$ & $\begin{array}{l}0.058876 \\
0.058978 \\
0.059082 \\
0.059186 \\
0.059288\end{array}$ & $\begin{array}{l}1172 \\
1174 \\
1176 \\
1178 \\
118 \mathrm{C}\end{array}$ & $\begin{array}{l}57.8 \\
57.9 \\
58.0 \\
58.1 \\
58.2\end{array}$ \\
\hline $\begin{array}{l}0.199208 \\
0.199577 \\
0.199945 \\
0.200314 \\
0.200683\end{array}$ & $\begin{array}{l}1182 \\
1184 \\
1186 \\
1188 \\
1190\end{array}$ & $\begin{array}{l}2.6 \\
2.6 \\
2.6 \\
2.6 \\
2.6\end{array}$ & $\begin{array}{l}2.155323 \\
2.161464 \\
2.167616 \\
2.173780 \\
2.179961\end{array}$ & $\begin{array}{l}1182 \\
1184 \\
1186 \\
1188 \\
1190\end{array}$ & $\begin{array}{l}26.5 \\
26.5 \\
26.6 \\
26.7 \\
26.7\end{array}$ & $\begin{array}{l}0.078572 \\
0.078650 \\
0.078729 \\
0.078808 \\
0.078886\end{array}$ & $\begin{array}{l}1182 \\
1184 \\
1186 \\
1188 \\
1190\end{array}$ & $\begin{array}{l}76.2 \\
76.2 \\
76.2 \\
76.3 \\
76.3\end{array}$ & $\begin{array}{l}0.059393 \\
0.059497 \\
0.059599 \\
0.059703 \\
0.059806\end{array}$ & $\begin{array}{l}1182 \\
1184 \\
1186 \\
1188 \\
1190\end{array}$ & $\begin{array}{l}58.3 \\
58.4 \\
58.5 \\
58.6 \\
58.7\end{array}$ \\
\hline $\begin{array}{l}0.201052 \\
0.201422 \\
0.201791 \\
0.202160 \\
0.202531\end{array}$ & $\begin{array}{l}1192 \\
1194 \\
1196 \\
1198 \\
1200\end{array}$ & $\begin{array}{l}2.6 \\
2.6 \\
2.7 \\
2.7 \\
2.7\end{array}$ & $\begin{array}{l}2.186146 \\
2.192346 \\
2.198562 \\
2.204786 \\
2.211023\end{array}$ & $\begin{array}{l}1192 \\
1194 \\
1196 \\
1198 \\
1200\end{array}$ & $\begin{array}{l}26.7 \\
26.8 \\
26.8 \\
26.9\end{array}$ & $\begin{array}{l}0.078965 \\
0.079043 \\
0.079123 \\
0.079202 \\
0.079280\end{array}$ & $\begin{array}{l}1192 \\
1194 \\
1196 \\
1198\end{array}$ & $\begin{array}{l}76.4 \\
76.4 \\
76.4 \\
76.5\end{array}$ & $\begin{array}{l}0.059909 \\
0.060013 \\
0.060117 \\
0.060220 \\
0.060324\end{array}$ & $\begin{array}{l}1192 \\
1194 \\
1196 \\
1198 \\
120 C\end{array}$ & $\begin{array}{l}58.8 \\
58.9 \\
59.0 \\
59.1 \\
59.2\end{array}$ \\
\hline
\end{tabular}


$1202-1300$

Geologic age, in millions of years, calculated from the atomic ratios of indicated isotopes

\begin{tabular}{|c|c|c|c|c|c|c|c|c|c|c|c|}
\hline \multicolumn{3}{|c|}{$N_{206} / N_{238}$} & \multicolumn{3}{|c|}{$N_{207} / N_{235}$} & \multicolumn{3}{|c|}{$N_{207} / N_{206}$} & \multicolumn{3}{|c|}{$N_{208} / N_{232}$} \\
\hline \multirow[b]{2}{*}{ Ratio } & \multicolumn{2}{|c|}{ Age } & \multirow[b]{2}{*}{ Ratio } & \multicolumn{2}{|c|}{ Age } & \multirow[b]{2}{*}{ Ratio } & \multicolumn{2}{|c|}{ Age } & \multirow[b]{2}{*}{ Ratio } & \multicolumn{2}{|c|}{ Age } \\
\hline & $\begin{array}{c}\text { Number } \\
\text { of } \\
\text { years }\end{array}$ & $\begin{array}{c}\text { Error } \\
\pm\end{array}$ & & \begin{tabular}{|c|}
$\begin{array}{c}\text { Number } \\
\text { of } \\
\text { years }\end{array}$ \\
\end{tabular} & $\begin{array}{c}\text { Error } \\
\pm\end{array}$ & & \begin{tabular}{|c|}
$\begin{array}{c}\text { Number } \\
\text { of } \\
\text { years }\end{array}$ \\
\end{tabular} & $\begin{array}{c}\text { Error } \\
\pm\end{array}$ & & \begin{tabular}{|c|}
$\begin{array}{c}\text { Numb:r } \\
\text { of } \\
\text { years }\end{array}$ \\
\end{tabular} & $\begin{array}{c}\text { Error } \\
\pm\end{array}$ \\
\hline $\begin{array}{l}0.202899 \\
0.203268 \\
0.203640 \\
0.204009 \\
0.204379\end{array}$ & $\begin{array}{l}1202 \\
1204 \\
1206 \\
1208 \\
1210\end{array}$ & $\begin{array}{l}2.7 \\
2.7 \\
2.7 \\
2.7 \\
2.7\end{array}$ & $\begin{array}{l}2.217275 \\
2.223534 \\
2.229809 \\
2.236095 \\
2.242392\end{array}$ & $\begin{array}{l}1202 \\
1204 \\
1206 \\
1208 \\
1210\end{array}$ & $\begin{array}{l}27.0 \\
27.0 \\
27.1 \\
27.1 \\
27.2\end{array}$ & $\begin{array}{l}9360 \\
9440 \\
9518 \\
9598 \\
9678\end{array}$ & $\begin{array}{l}1202 \\
1204 \\
1206 \\
1208 \\
1210\end{array}$ & $\begin{array}{l}76.5 \\
76.6 \\
76.6 \\
76.7 \\
76.7\end{array}$ & $\begin{array}{l}427 \\
531 \\
633 \\
738 \\
842\end{array}$ & $\begin{array}{l}1202 \\
120^{4} \\
1205 \\
120^{\circ} \\
1210\end{array}$ & $\begin{array}{l}59.4 \\
59.5 \\
59.5 \\
59.6\end{array}$ \\
\hline $\begin{array}{l}0.204751 \\
0.205120 \\
0.205490 \\
0.205862 \\
0.206231\end{array}$ & $\begin{array}{l}1212 \\
1214 \\
1216 \\
1218 \\
1220\end{array}$ & $\begin{array}{l}2.7 \\
2.7 \\
2.7 \\
2.7 \\
2.7\end{array}$ & $\begin{array}{l}2.248701 \\
2.255027 \\
2.261361 \\
2.267705 \\
2.274067\end{array}$ & $\begin{array}{l}1212 \\
1214 \\
1216 \\
1218 \\
1220\end{array}$ & $\begin{array}{l}27.2 \\
27.2 \\
27.3 \\
27.3 \\
27.4\end{array}$ & $\begin{array}{l}57 \\
338 \\
18 \\
997 \\
78\end{array}$ & $\begin{array}{l}1212 \\
1214 \\
1216 \\
1218 \\
1220\end{array}$ & $\begin{array}{l}76.7 \\
76.8 \\
76.8 \\
76.8 \\
76.9\end{array}$ & $\begin{array}{l}0945 \\
1048 \\
1152 \\
1256 \\
1360\end{array}$ & $\begin{array}{l}1212 \\
1214 \\
1216 \\
1218 \\
1220\end{array}$ & $\begin{array}{l}59.7 \\
59.8 \\
59.9 \\
60.0 \\
60.1\end{array}$ \\
\hline $\begin{array}{l}0.206603 \\
0.206974 \\
0.207345 \\
0.207716 \\
0.208088\end{array}$ & $\begin{array}{l}1222 \\
1224 \\
1226 \\
1228 \\
1230\end{array}$ & $\begin{array}{l}2.7 \\
2.7 \\
2.7 \\
2.7 \\
2.7\end{array}$ & $\begin{array}{l}439 \\
824 \\
221 \\
221 \\
630 \\
051\end{array}$ & $\begin{array}{l}1222 \\
1224 \\
1226 \\
1228 \\
1230\end{array}$ & $\begin{array}{l}27.4 \\
27.5 \\
27.5 \\
27.6 \\
27.6\end{array}$ & $\begin{array}{l}158 \\
238 \\
319 \\
399 \\
479\end{array}$ & $\begin{array}{l}1222 \\
1224 \\
1226 \\
1228 \\
1230\end{array}$ & $\begin{array}{l}76.9 \\
76.9 \\
77.0 \\
77.0 \\
77.0\end{array}$ & $\begin{array}{l}463 \\
.566 \\
.670 \\
773 \\
878\end{array}$ & $\begin{array}{l}1222 \\
1224 \\
1226 \\
1228 \\
1230\end{array}$ & $\begin{array}{l}60 \\
60 \\
60 \\
60 \\
60\end{array}$ \\
\hline $\begin{array}{l}0.208460 \\
0.208830 \\
0.209203 \\
0.209575 \\
0.209945\end{array}$ & $\begin{array}{l}1232 \\
1234 \\
1236 \\
1238 \\
1240\end{array}$ & $\begin{array}{l}2.7 \\
2.7 \\
2.7 \\
2.7 \\
2.7\end{array}$ & $\begin{array}{l}2.312482 \\
2.318934 \\
2.325392 \\
2.331860 \\
2.338347\end{array}$ & $\begin{array}{l}1232 \\
1234 \\
1236 \\
1238 \\
1240\end{array}$ & $\begin{array}{l}27.6 \\
27.7 \\
27.7 \\
27.8 \\
27.8\end{array}$ & $\begin{array}{l}560 \\
642 \\
722 \\
803 \\
885\end{array}$ & $\begin{array}{l}1232 \\
1234 \\
1236 \\
1238 \\
1240\end{array}$ & $\begin{array}{l}77 \\
77 \\
77 \\
77 \\
77\end{array}$ & & $\begin{array}{l}1232 \\
1234 \\
1235 \\
1239 \\
1240\end{array}$ & $\begin{array}{l}60.8 \\
60.9 \\
61.0 \\
61.1\end{array}$ \\
\hline $\begin{array}{l}0.210319 \\
0.210690 \\
0.211062 \\
0.211435 \\
0.211806\end{array}$ & $\begin{array}{l}1242 \\
1244 \\
1246 \\
1248 \\
1250\end{array}$ & $\begin{array}{l}2.8 \\
2.8 \\
2.8 \\
2.8 \\
2.8\end{array}$ & $\begin{array}{l}844 \\
355 \\
878 \\
412 \\
959\end{array}$ & $\begin{array}{l}1242 \\
1244 \\
1246 \\
1248 \\
1250\end{array}$ & $\begin{array}{l}27.9 \\
27.9 \\
28.0 \\
28.0 \\
28.1\end{array}$ & $\begin{array}{l}0.080965 \\
0.081047 \\
0.081129 \\
0.081210 \\
0.081292\end{array}$ & $\begin{array}{l}1242 \\
1244 \\
1246 \\
1248 \\
1250\end{array}$ & $\begin{array}{l}77.3 \\
77.3 \\
77.4 \\
77.4 \\
77.4\end{array}$ & & $\begin{array}{l}1242 \\
1244 \\
1246 \\
1248 \\
1250\end{array}$ & $\begin{array}{l}61.2 \\
61.3 \\
61.4 \\
61.5 \\
61.6\end{array}$ \\
\hline $\begin{array}{l}0.212181 \\
0.212553 \\
0.212924 \\
0.213298 \\
0.213671\end{array}$ & $\begin{array}{l}1252 \\
1254 \\
1256 \\
1258 \\
1260\end{array}$ & $\begin{array}{l}2.8 \\
2.8 \\
2.8 \\
2.8 \\
2.8\end{array}$ & $\begin{array}{l}2.377523 \\
2.384092 \\
2.390678 \\
2.397279 \\
2.403892\end{array}$ & $\begin{array}{l}1252 \\
1254 \\
1256 \\
1258 \\
1260\end{array}$ & $\begin{array}{l}28.1 \\
28.1 \\
28.2 \\
28.2 \\
28.3\end{array}$ & $\begin{array}{l}1373 \\
1455 \\
1538 \\
1620 \\
1702\end{array}$ & $\begin{array}{l}1252 \\
1254 \\
1256 \\
1258 \\
1260\end{array}$ & $\begin{array}{l}77.5 \\
77.5 \\
77.5 \\
77.6 \\
77.6\end{array}$ & $\begin{array}{l}019 \\
122 \\
226 \\
330 \\
433\end{array}$ & $\begin{array}{l}1252 \\
1254 \\
1256 \\
1258 \\
1260\end{array}$ & $\begin{array}{l}61 \\
61 \\
61 \\
62\end{array}$ \\
\hline $\begin{array}{l}0.214045 \\
0.214417 \\
0.214790 \\
0.215165 \\
0.215538\end{array}$ & $\begin{array}{l}1262 \\
1264 \\
1266 \\
1268 \\
1270\end{array}$ & $\begin{array}{l}2.8 \\
2.8 \\
2.8 \\
2.8 \\
2.8\end{array}$ & $\begin{array}{l}2.410516 \\
2.417156 \\
2.423804 \\
2.430469 \\
2.437145\end{array}$ & $\begin{array}{l}1262 \\
1264 \\
1266 \\
1268 \\
1270\end{array}$ & $\begin{array}{l}28.3 \\
28.4 \\
28.4 \\
28.5 \\
28.5\end{array}$ & $\begin{array}{l}81784 \\
81867 \\
81950 \\
82032 \\
82115\end{array}$ & $\begin{array}{l}1262 \\
1264 \\
1266 \\
1268 \\
1270\end{array}$ & $\begin{array}{l}77 \\
77 \\
77 \\
77 \\
77\end{array}$ & $\begin{array}{l}537 \\
642 \\
745 \\
849 \\
952\end{array}$ & $\begin{array}{l}1262 \\
1264 \\
1266 \\
1268 \\
1270\end{array}$ & $\begin{array}{l}\text { OL. } \\
62.4 \\
62.5 \\
62.6\end{array}$ \\
\hline $\begin{array}{l}0.215911 \\
0.216286 \\
0.216658 \\
0.217033 \\
0.217407\end{array}$ & $\begin{array}{l}1272 \\
1274 \\
1276 \\
1278 \\
1280\end{array}$ & $\begin{array}{l}2.8 \\
2.8 \\
2.8 \\
2.8 \\
2.8\end{array}$ & $\begin{array}{l}2.443833 \\
2.450534 \\
2.457254 \\
2.463980 \\
2.470720\end{array}$ & $\begin{array}{l}1272 \\
1274 \\
1276 \\
1278 \\
1280\end{array}$ & $\begin{array}{l}28.5 \\
28.6 \\
28.6 \\
28.7 \\
28.7\end{array}$ & $\begin{array}{l}0.082198 \\
0.082280 \\
0.082364 \\
0.082447 \\
0.082530\end{array}$ & $\begin{array}{l}1272 \\
1274 \\
1276 \\
1278 \\
1280\end{array}$ & $\begin{array}{l}77.9 \\
77.9 \\
77.9 \\
78.0 \\
78.0\end{array}$ & & $\begin{array}{l}1272 \\
1274 \\
1276 \\
1278 \\
1280\end{array}$ & $\begin{array}{l}62.7 \\
62.8 \\
62.9 \\
63.0 \\
63.1\end{array}$ \\
\hline $\begin{array}{l}0.217780 \\
0.218156 \\
0.218530 \\
0.218905 \\
0.219279\end{array}$ & $\begin{array}{l}1282 \\
1284 \\
1286 \\
1288 \\
1290\end{array}$ & $\begin{array}{l}2.8 \\
2.8 \\
2.9 \\
2.9 \\
2.9\end{array}$ & $\begin{array}{l}2.477473 \\
2.484244 \\
2.491026 \\
2.497817 \\
2.504627\end{array}$ & $\begin{array}{l}1282 \\
1284 \\
1286 \\
1288 \\
1290\end{array}$ & $\begin{array}{l}28.8 \\
28.8 \\
28.9 \\
28.9 \\
28.9\end{array}$ & $\begin{array}{l}0.082614 \\
0.082697 \\
0.082781 \\
0.082864 \\
0.082949\end{array}$ & $\begin{array}{l}1282 \\
1284 \\
1286 \\
1288 \\
1290\end{array}$ & $\begin{array}{l}78.0 \\
78.1 \\
78.1 \\
78.1 \\
78.1\end{array}$ & $\begin{array}{l}4576 \\
64681 \\
4785 \\
6888 \\
64992\end{array}$ & $\begin{array}{l}1282 \\
1284 \\
1285 \\
1288 \\
1250\end{array}$ & $\begin{array}{l}63.2 \\
63.3 \\
63.4 \\
63.5 \\
63.6\end{array}$ \\
\hline $\begin{array}{l}0.219653 \\
0.220030 \\
0.220405 \\
0.220779 \\
0.221155\end{array}$ & $\begin{array}{l}1292 \\
1294 \\
1296 \\
1298 \\
1300\end{array}$ & $\begin{array}{l}2.9 \\
2.9 \\
2.9 \\
2.9 \\
2.9\end{array}$ & $\begin{array}{l}2.511448 \\
2.518281 \\
2.525129 \\
2.531988 \\
2.538864\end{array}$ & $\begin{array}{l}1292 \\
1294 \\
1296 \\
1298 \\
1300\end{array}$ & $\begin{array}{l}29.0 \\
29.0 \\
29.1 \\
29.1 \\
29.2\end{array}$ & $\begin{array}{l}0.083033 \\
0.083116 \\
0.083200 \\
0.083285 \\
0.083369\end{array}$ & $\begin{array}{l}1292 \\
1294 \\
1296 \\
1298 \\
1300\end{array}$ & $\begin{array}{l}78.3 \\
78.3 \\
78.3 \\
78.4 \\
78.4\end{array}$ & $\begin{array}{l}0.065095 \\
0.065199 \\
0.065305 \\
0.065408 \\
0.065512\end{array}$ & $\begin{array}{l}12 \% 2 \\
1254 \\
12 \% 5 \\
12 \% \% \\
13 c 0\end{array}$ & $\begin{array}{l}63.7 \\
63.8 \\
63.9 \\
64.0\end{array}$ \\
\hline
\end{tabular}


$1302-1400$

Geologic age, in millions of years, calculated from the atomic ratios of indicated isotopes

\begin{tabular}{|c|c|c|c|c|c|c|c|c|c|c|c|}
\hline \multicolumn{3}{|c|}{$N_{206} / N_{238}$} & \multicolumn{3}{|c|}{$N_{207} / N_{235}$} & \multicolumn{3}{|c|}{$N_{207} / N_{206}$} & \multicolumn{3}{|c|}{$N_{208} / N_{232}$} \\
\hline \multirow[b]{2}{*}{ Ratio } & \multicolumn{2}{|c|}{ Age } & \multirow[b]{2}{*}{ Ratio } & \multicolumn{2}{|c|}{ Age } & \multirow[b]{2}{*}{ Ratio } & \multicolumn{2}{|c|}{ Age } & \multirow[b]{2}{*}{ Ratio } & \multicolumn{2}{|c|}{ Age } \\
\hline & \begin{tabular}{|c|}
$\begin{array}{c}\text { Number } \\
\text { of } \\
\text { years }\end{array}$ \\
\end{tabular} & $\begin{array}{c}\text { Error } \\
\pm\end{array}$ & & \begin{tabular}{|c|}
$\begin{array}{c}\text { Number } \\
\text { of } \\
\text { years }\end{array}$ \\
\end{tabular} & $\begin{array}{c}\text { Error } \\
\pm\end{array}$ & & \begin{tabular}{|c|}
$\begin{array}{c}\text { Number } \\
\text { of } \\
\text { years }\end{array}$ \\
\end{tabular} & $\begin{array}{c}\text { Error } \\
\pm \\
\end{array}$ & & \begin{tabular}{|c|}
$\begin{array}{c}\text { Number } \\
\text { of } \\
\text { years }\end{array}$ \\
\end{tabular} & $\begin{array}{c}\text { Error } \\
\pm\end{array}$ \\
\hline $\begin{array}{l}0.221530 \\
0.221906 \\
0.222281 \\
0.222656 \\
0.223034\end{array}$ & $\begin{array}{l}1302 \\
1304 \\
1306 \\
1308 \\
1310\end{array}$ & $\begin{array}{l}2.9 \\
2.9 \\
2.9 \\
2.9 \\
2.9\end{array}$ & $\begin{array}{l}2.545752 \\
2.552653 \\
2.559564 \\
2.566495 \\
2.573437\end{array}$ & $\begin{array}{l}1302 \\
1304 \\
1306 \\
1308 \\
1310\end{array}$ & $\begin{array}{l}29.3 \\
29.3 \\
29.4 \\
29.4\end{array}$ & $\begin{array}{l}0.083454 \\
0.083538 \\
0.083623 \\
0.083708 \\
0.083793\end{array}$ & $\begin{array}{l}1302 \\
1304 \\
1306 \\
1308 \\
1310\end{array}$ & $\begin{array}{l}78.4 \\
78.5 \\
78.5 \\
78.5 \\
78.6\end{array}$ & $\begin{array}{l}0.065616 \\
0.065719 \\
0.065825 \\
0.065928 \\
0.066032\end{array}$ & $\begin{array}{l}1302 \\
1304 \\
1306 \\
1308 \\
1310\end{array}$ & $\begin{array}{l}64.2 \\
64.3 \\
64.4 \\
64.5 \\
64.6\end{array}$ \\
\hline $\begin{array}{l}0.223408 \\
0.223786 \\
0.224161 \\
0.224538 \\
0.224914\end{array}$ & $\begin{array}{l}1312 \\
1314 \\
1316 \\
1318 \\
1320\end{array}$ & $\begin{array}{l}2.9 \\
2.9 \\
2.9 \\
2.9 \\
2.9\end{array}$ & $\begin{array}{l}2.580388 \\
2.587360 \\
2.594338 \\
2.601335 \\
2.608345\end{array}$ & $\begin{array}{l}1312 \\
1314 \\
1316 \\
1318 \\
1320\end{array}$ & $\begin{array}{l}29.4 \\
29.5 \\
29.5 \\
29.6 \\
29.6\end{array}$ & $\begin{array}{l}0.083878 \\
0.083963 \\
0.084049 \\
0.084134 \\
0.084219\end{array}$ & $\begin{array}{l}1312 \\
1314 \\
1316 \\
1318 \\
1320\end{array}$ & $\begin{array}{l}78.6 \\
78.6 \\
78.7 \\
78.7 \\
78.8\end{array}$ & $\begin{array}{l}136 \\
239 \\
345 \\
449 \\
552\end{array}$ & $\begin{array}{l}1312 \\
1314 \\
1316 \\
1318 \\
1320\end{array}$ & $\begin{array}{l}64.7 \\
64.8 \\
64.9 \\
65.0 \\
65.1\end{array}$ \\
\hline $\begin{array}{l}0.225290 \\
0.225667 \\
0.226044 \\
0.226421 \\
0.226798\end{array}$ & $\begin{array}{l}1322 \\
1324 \\
1326 \\
1328 \\
1330\end{array}$ & $\begin{array}{l}2.9 \\
2.9 \\
2.9 \\
2.9 \\
2.9\end{array}$ & $\begin{array}{l}2.615367 \\
2.622403 \\
2.629455 \\
2.636516 \\
2.643593\end{array}$ & $\begin{array}{l}1322 \\
1324 \\
1326 \\
1328 \\
1330\end{array}$ & $\begin{array}{l}29.7 \\
29.7 \\
29.8 \\
29.8 \\
29.8\end{array}$ & $\begin{array}{l}0.084305 \\
0.084391 \\
0.084477 \\
0.084562 \\
0.084648\end{array}$ & $\begin{array}{l}1322 \\
1324 \\
1326 \\
1328 \\
1330\end{array}$ & $\begin{array}{l}78.8 \\
78.9 \\
78.9 \\
78.9 \\
78.9\end{array}$ & $\begin{array}{l}0.066657 \\
0.066760 \\
0.066865 \\
0.066970 \\
0.067073\end{array}$ & $\begin{array}{l}1322 \\
1324 \\
1326 \\
1328 \\
1330\end{array}$ & $\begin{array}{l}65.2 \\
65.3 \\
65.4 \\
65.5 \\
65.6\end{array}$ \\
\hline $\begin{array}{l}0.227175 \\
0.227552 \\
0.227931 \\
0.228308 \\
0.228686\end{array}$ & $\begin{array}{l}1332 \\
1334 \\
1336 \\
1338 \\
1340\end{array}$ & $\begin{array}{l}3.0 \\
3.0 \\
3.0 \\
3.0 \\
3.0\end{array}$ & $\begin{array}{l}2.650684 \\
2.657792 \\
2.664906 \\
2.672041 \\
2.679188\end{array}$ & $\begin{array}{l}1332 \\
1334 \\
1336 \\
1338 \\
1340\end{array}$ & $\begin{array}{l}29.9 \\
29.9 \\
30.0 \\
30.0 \\
30.1\end{array}$ & $\begin{array}{l}0.084735 \\
0.084821 \\
0.084907 \\
0.084993 \\
0.085080\end{array}$ & $\begin{array}{l}1332 \\
1334 \\
1336 \\
1338 \\
1340\end{array}$ & $\begin{array}{l}79.0 \\
79.0 \\
79.1 \\
79.1 \\
79.1\end{array}$ & $\begin{array}{l}0.067177 \\
0.067283 \\
0.067386 \\
0,067490 \\
0,067594\end{array}$ & $\begin{array}{l}1332 \\
1334 \\
1336 \\
1338 \\
1340\end{array}$ & $\begin{array}{l}65.7 \\
65.8 \\
65.9 \\
66.0 \\
66.1\end{array}$ \\
\hline $\begin{array}{l}0.229064 \\
0.229440 \\
0.229820 \\
0.230196 \\
0.230575\end{array}$ & $\begin{array}{l}1342 \\
1344 \\
1346 \\
1348 \\
1350\end{array}$ & $\begin{array}{l}3.0 \\
3.0 \\
3.0 \\
3.0 \\
3.0\end{array}$ & $\begin{array}{l}2.686349 \\
2.693522 \\
2.700714 \\
2.707915 \\
2.715129\end{array}$ & $\begin{array}{l}1342 \\
1344 \\
1346 \\
1348 \\
1350\end{array}$ & $\begin{array}{l}30.1 \\
30.2 \\
30.2 \\
30.2 \\
30.3\end{array}$ & $\begin{array}{l}0.085167 \\
0.085254 \\
0.085340 \\
0.085428 \\
0.085515\end{array}$ & $\begin{array}{l}1342 \\
1344 \\
1346 \\
1348 \\
1350\end{array}$ & $\begin{array}{l}79.2 \\
79.2 \\
79.3 \\
79.3 \\
79.3\end{array}$ & $\begin{array}{l}698 \\
804 \\
907 \\
011 \\
116\end{array}$ & $\begin{array}{l}1342 \\
1344 \\
1346 \\
1348 \\
1350\end{array}$ & $\begin{array}{l}66.2 \\
66.3 \\
66.4 \\
66.5 \\
66.5\end{array}$ \\
\hline $\begin{array}{l}0.230954 \\
0.231332 \\
0.231711 \\
0.232090 \\
0.232467\end{array}$ & $\begin{array}{l}1352 \\
1354 \\
1356 \\
1358 \\
1360\end{array}$ & $\begin{array}{l}3.0 \\
3.0 \\
3.0 \\
3.0 \\
3.0\end{array}$ & $\begin{array}{l}2.722362 \\
2.729607 \\
2.736863 \\
2.744136 \\
2.751424\end{array}$ & $\begin{array}{l}1352 \\
1354 \\
1356 \\
1358 \\
1360\end{array}$ & $\begin{array}{l}30.3 \\
30.4 \\
30.4 \\
30.5 \\
30.5\end{array}$ & $\begin{array}{l}0.085602 \\
0.085690 \\
0.085777 \\
0.085864 \\
0.085953\end{array}$ & $\begin{array}{l}1352 \\
1354 \\
1356 \\
1358 \\
1360\end{array}$ & $\begin{array}{l}79.4 \\
79.4 \\
79.4 \\
79.5 \\
79.5\end{array}$ & $\begin{array}{l}219 \\
325 \\
428 \\
533 \\
637\end{array}$ & $\begin{array}{l}1352 \\
1354 \\
1356 \\
1358 \\
1360\end{array}$ & $\begin{array}{l}66.6 \\
66.7 \\
66.8 \\
66.9 \\
67.0\end{array}$ \\
\hline $\begin{array}{l}0.232847 \\
0.233226 \\
0.233604 \\
0.233984 \\
0.234363\end{array}$ & $\begin{array}{l}1362 \\
1364 \\
1366 \\
1368 \\
1370\end{array}$ & $\begin{array}{l}3.0 \\
3.0 \\
3.0 \\
3.0 \\
3.0\end{array}$ & $\begin{array}{l}2.758724 \\
2.766041 \\
2.773370 \\
2.780711 \\
2.788072\end{array}$ & $\begin{array}{l}1362 \\
1364 \\
1366 \\
1368 \\
1370\end{array}$ & $\begin{array}{l}30.6 \\
30.6 \\
30.7 \\
30.7 \\
30.7\end{array}$ & $\begin{array}{l}0.086040 \\
0.086128 \\
0.086217 \\
0.086304 \\
0.086393\end{array}$ & $\begin{array}{l}1362 \\
1364 \\
1366 \\
1368 \\
1370\end{array}$ & $\begin{array}{l}79.6 \\
79.6 \\
79.6 \\
79.7 \\
79.7\end{array}$ & $\begin{array}{l}740 \\
846 \\
951 \\
054 \\
159\end{array}$ & $\begin{array}{l}1362 \\
1364 \\
1366 \\
1368 \\
1370\end{array}$ & $\begin{array}{l}67.1 \\
67.2 \\
67.3 \\
67.4 \\
67.5\end{array}$ \\
\hline $\begin{array}{l}0.234744 \\
0.235122 \\
0.235502 \\
0.235882 \\
0.236262\end{array}$ & $\begin{array}{l}1372 \\
1374 \\
1376 \\
1378 \\
1380\end{array}$ & $\begin{array}{l}3.0 \\
3.0 \\
3.1 \\
3.1 \\
3.1\end{array}$ & $\begin{array}{l}2.795445 \\
2.802831 \\
2.810232 \\
2.817649 \\
2.825076\end{array}$ & $\begin{array}{l}1372 . \\
1374 \\
1376 \\
1378 \\
1380\end{array}$ & $\begin{array}{l}30.8 \\
30.8 \\
30.9 \\
30.9 \\
31.0\end{array}$ & $\begin{array}{l}0,086481 \\
0.086570 \\
0,086658 \\
0.086747 \\
0.086836\end{array}$ & $\begin{array}{l}1372 \\
1374 \\
1376 \\
1378 \\
1380\end{array}$ & $\begin{array}{l}79.7 \\
79.8 \\
79.8 \\
79.9 \\
79.9\end{array}$ & $\begin{array}{l}0,069263 \\
0,069368 \\
0.069472 \\
0.069576 \\
0,069680\end{array}$ & $\begin{array}{l}1372 \\
1374 \\
1376 \\
1378 \\
1380\end{array}$ & $\begin{array}{l}67.6 \\
67.7 \\
67.8 \\
67.9 \\
68.0\end{array}$ \\
\hline $\begin{array}{l}0.236643 \\
0.237022 \\
0.237401 \\
0.237783 \\
0.238163\end{array}$ & $\begin{array}{l}1382 \\
1384 \\
1386 \\
1388 \\
1390\end{array}$ & $\begin{array}{l}3.1 \\
3.1 \\
3.1 \\
3.1 \\
3.1\end{array}$ & $\begin{array}{l}2.832521 \\
2.839981 \\
2.847454 \\
2.854939 \\
2.862445\end{array}$ & $\begin{array}{l}1382 \\
1384 \\
1386 \\
1388 \\
1390\end{array}$ & $\begin{array}{l}31.0 \\
31.1 \\
31.1 \\
31.1 \\
31.2\end{array}$ & $\begin{array}{l}0.086925 \\
0.087014 \\
0.087104 \\
0.087193 \\
0.087282\end{array}$ & $\begin{array}{l}1382 \\
1384 \\
1386 \\
1388 \\
1390\end{array}$ & $\begin{array}{l}79.9 \\
80.0 \\
80.0 \\
80.1 \\
80,1\end{array}$ & $\begin{array}{l}0.069786 \\
0.069889 \\
0.069994 \\
0.070098 \\
0.070203\end{array}$ & $\begin{array}{l}1382 \\
1384 \\
1386 \\
1388 \\
1390\end{array}$ & $\begin{array}{l}68.1 \\
68.2 \\
68.3 \\
68.4 \\
68.5\end{array}$ \\
\hline $\begin{array}{l}0.238543 \\
0.238924 \\
0.239306 \\
0.239687 \\
0.240068\end{array}$ & $\begin{array}{l}1392 \\
1394 \\
1396 \\
1398 \\
1400\end{array}$ & $\begin{array}{l}3.1 \\
3.1 \\
3.1 \\
3.1 \\
3.1\end{array}$ & $\begin{array}{l}2.869960 \\
2.877491 \\
2.885039 \\
2.892600 \\
2.900174\end{array}$ & $\begin{array}{l}1392 \\
1394 \\
1396 \\
1398 \\
1400\end{array}$ & $\begin{array}{l}31.2 \\
31.3 \\
31.3 \\
31.4 \\
31.4\end{array}$ & $\begin{array}{l}0.087372 \\
0.087462 \\
0.087551 \\
0.087641 \\
0.087731\end{array}$ & $\begin{array}{l}1392 \\
1394 \\
1396 \\
1398 \\
1400\end{array}$ & $\begin{array}{l}80,1 \\
80.2 \\
80.2 \\
80.2 \\
80.3\end{array}$ & $\begin{array}{l}0.070308 \\
0.070412 \\
0.070516 \\
0.070621 \\
0.070726\end{array}$ & $\begin{array}{l}1392 \\
1394 \\
1396 \\
1398 \\
1400\end{array}$ & $\begin{array}{l}68.6 \\
68.7 \\
68.8 \\
68.9 \\
69.0\end{array}$ \\
\hline
\end{tabular}


$1402-1500$

Geologic age, in millions of years, calculated from the atomic ratios of indicated isotopes

\begin{tabular}{|c|c|c|c|c|c|c|c|c|c|c|c|}
\hline \multicolumn{3}{|c|}{$N_{206} / N_{238}$} & \multicolumn{3}{|c|}{$N_{207} / N_{235}$} & \multicolumn{3}{|c|}{$N_{207} / N_{206}$} & \multicolumn{3}{|c|}{$N_{208} / N_{232}$} \\
\hline \multirow[b]{2}{*}{ Ratio } & \multicolumn{2}{|c|}{ Age } & \multirow[b]{2}{*}{ Ratio } & \multicolumn{2}{|c|}{ Age } & \multirow[b]{2}{*}{ Ratio } & \multicolumn{2}{|c|}{ Age } & \multirow[b]{2}{*}{ Ratio } & \multicolumn{2}{|c|}{ Age } \\
\hline & $\begin{array}{c}\begin{array}{c}\text { Number } \\
\text { of } \\
\text { years }\end{array} \\
\end{array}$ & $\begin{array}{c}\text { Error } \\
\pm\end{array}$ & & $\begin{array}{c}\text { Number } \\
\text { of } \\
\text { years }\end{array}$ & $\begin{array}{c}\text { Error } \\
\pm\end{array}$ & & \begin{tabular}{|c|}
$\begin{array}{c}\text { Number } \\
\text { of } \\
\text { years }\end{array}$ \\
\end{tabular} & $\begin{array}{c}\text { Error } \\
\pm\end{array}$ & & $\begin{array}{c}\text { Number } \\
\text { of } \\
\text { years }\end{array}$ & $\begin{array}{c}\text { Error } \\
\pm\end{array}$ \\
\hline $\begin{array}{l}0.240448 \\
0.240831 \\
0.241211 \\
0.241594 \\
0.241975\end{array}$ & $\begin{array}{l}1402 \\
1404 \\
1406 \\
1408 \\
1410\end{array}$ & $\begin{array}{l}3.1 \\
3.1 \\
3.1 \\
3.1 \\
3.1\end{array}$ & $\begin{array}{l}767 \\
372 \\
991 \\
630 \\
278\end{array}$ & $\begin{array}{l}1402 \\
1404 \\
1406 \\
1408 \\
1410\end{array}$ & $\begin{array}{l}31.5 \\
31.5 \\
31.6 \\
31.6 \\
31.6\end{array}$ & $\begin{array}{l}7822 \\
7911 \\
8002 \\
8092 \\
8183\end{array}$ & $\begin{array}{l}1402 \\
1404 \\
1406 \\
1408 \\
1410\end{array}$ & $\begin{array}{l}80.4 \\
80.4 \\
80.4 \\
80.5\end{array}$ & $\begin{array}{l}0.070830 \\
0.070934 \\
0.071039 \\
0.071144 \\
0.071248\end{array}$ & $\begin{array}{l}1402 \\
140 a \\
1406 \\
1408 \\
1410\end{array}$ & $\begin{array}{l}69.1 \\
69.2 \\
69.3 \\
69.4 \\
69.5\end{array}$ \\
\hline $\begin{array}{l}0.242357 \\
0.242738 \\
0.243121 \\
0.243503 \\
0.243886\end{array}$ & $\begin{array}{l}1412 \\
1414 \\
1416 \\
1418 \\
1420\end{array}$ & $\begin{array}{l}3.1 \\
3.1 \\
3.1 \\
3.1 \\
3.1\end{array}$ & $\begin{array}{l}41 \\
24 \\
15 \\
24 \\
50\end{array}$ & $\begin{array}{l}1412 \\
1414 \\
1416 \\
1418 \\
1420\end{array}$ & $\begin{array}{l}31.7 \\
31.8 \\
31.8 \\
31.9\end{array}$ & $\begin{array}{r}74 \\
365 \\
456 \\
47 \\
38\end{array}$ & $\begin{array}{l}1412 \\
1414 \\
1416 \\
1418 \\
1420\end{array}$ & $\begin{array}{l}80.6 \\
80.6 \\
80.6 \\
80.7\end{array}$ & $\begin{array}{l}0.071353 \\
0.071458 \\
0.071562 \\
0.071667 \\
0.071771\end{array}$ & $\begin{array}{l}1412 \\
1414 \\
1416 \\
1418 \\
1420\end{array}$ & $\begin{array}{l}69.6 \\
69.7 \\
69.8 \\
69.9 \\
70.0\end{array}$ \\
\hline $\begin{array}{l}0.244268 \\
0.244652 \\
0.245034 \\
0.245415 \\
0.245800\end{array}$ & $\begin{array}{l}1422 \\
1424 \\
1426 \\
1428 \\
1430\end{array}$ & $\begin{array}{l}3.2 \\
3.2 \\
3.2 \\
3.2 \\
3.2\end{array}$ & $\left\{\begin{array}{l}89 \\
44 \\
15 \\
00 \\
997\end{array}\right.$ & $\begin{array}{l}1422 \\
1424 \\
1426 \\
1428 \\
1430\end{array}$ & $\begin{array}{l}32.0 \\
32.0 \\
32.0 \\
32.1\end{array}$ & $\begin{array}{l}29 \\
20 \\
12 \\
04 \\
95\end{array}$ & $\begin{array}{l}1422 \\
1424 \\
1426 \\
1428 \\
1430\end{array}$ & $\begin{array}{l}8 \\
8\end{array}$ & $\begin{array}{l}76 \\
80 \\
85 \\
90 \\
95\end{array}$ & $\begin{array}{l}1422 \\
1424 \\
1426 \\
1428 \\
1430\end{array}$ & $\begin{array}{l}70 \\
70 \\
70 \\
70\end{array}$ \\
\hline $\begin{array}{l}0.246181 \\
0.246566 \\
0.246948 \\
0.247332 \\
0.247715\end{array}$ & $\begin{array}{l}1432 \\
1434 \\
1436 \\
1438 \\
1440\end{array}$ & $\begin{array}{l}3.2 \\
3.2 \\
3.2 \\
3.2 \\
3.2\end{array}$ & $\left\{\begin{array}{l}11 \\
45 \\
90 \\
49 \\
329\end{array}\right.$ & $\begin{array}{l}1432 \\
1434 \\
1436 \\
1438 \\
1440\end{array}$ & $\begin{array}{l}32.1 \\
32.2 \\
32.2 \\
32.3 \\
32.3\end{array}$ & $\begin{array}{l}88 \\
79 \\
72 \\
764 \\
57\end{array}$ & $\begin{array}{l}1432 \\
1434 \\
1436 \\
1438 \\
1440\end{array}$ & & $\begin{array}{l}99 \\
04 \\
09 \\
14 \\
19\end{array}$ & $\begin{array}{l}1432 \\
1434 \\
1436 \\
1438 \\
1440\end{array}$ & $\begin{array}{l}7 \\
7 \\
7 \\
7 \\
7\end{array}$ \\
\hline $\begin{array}{l}0.248099 \\
0.248482 \\
0.248866 \\
0.249250 \\
0.249634\end{array}$ & $\begin{array}{l}1442 \\
1444 \\
1446 \\
1448 \\
1450\end{array}$ & $\begin{array}{l}3.2 \\
3.2 \\
3.2 \\
3.2 \\
3.2\end{array}$ & $\begin{array}{l}19 \\
25 \\
522 \\
86 \\
38\end{array}$ & $\begin{array}{l}1442 \\
1444 \\
1446 \\
1448 \\
1450\end{array}$ & $\begin{array}{l}32 \\
32 \\
32 \\
32 \\
32\end{array}$ & & $\begin{array}{l}1442 \\
1444 \\
1446 \\
1448 \\
1450\end{array}$ & & $\begin{array}{l}24 \\
29 \\
34 \\
37 \\
43\end{array}$ & $\begin{array}{l}1442 \\
1444 \\
1446 \\
1448 \\
1450\end{array}$ & $\begin{array}{l}71 . \\
71 \\
71 . \\
71 \\
71 .\end{array}$ \\
\hline $\begin{array}{l}0.250019 \\
0.250401 \\
0.250787 \\
0.251172 \\
0.251557\end{array}$ & $\begin{array}{l}1452 \\
1454 \\
1456 \\
1458 \\
1460\end{array}$ & $\begin{array}{l}3.2 \\
3.2 \\
3.2 \\
3.2 \\
3.2\end{array}$ & $\begin{array}{l}3.102410 \\
3.110393 \\
3.118389 \\
3.126410 \\
3.134440\end{array}$ & $\begin{array}{l}1452 \\
1454 \\
1456 \\
1458 \\
1460\end{array}$ & $\begin{array}{l}32.6 \\
32.6 \\
32.7 \\
32.7 \\
32.8\end{array}$ & $\begin{array}{l}13 \\
08 \\
00 \\
94 \\
87\end{array}$ & $\begin{array}{l}1452 \\
1454 \\
1456 \\
1458 \\
1460\end{array}$ & 3 & $\begin{array}{l}48 \\
52 \\
57 \\
62 \\
67\end{array}$ & $\begin{array}{l}1452 \\
145 \AA \\
1456 \\
1458 \\
1460\end{array}$ & $\begin{array}{l}71 \\
71 \\
71 \\
71 \\
72\end{array}$ \\
\hline $\begin{array}{l}0.251941 \\
0.252325 \\
0.252711 \\
0.253095 \\
0.253480\end{array}$ & $\begin{array}{l}1462 \\
1464 \\
1466 \\
1468 \\
1470\end{array}$ & $\begin{array}{l}3.2 \\
3.2 \\
3.3 \\
3.3 \\
3.3\end{array}$ & $\begin{array}{l}484 \\
548 \\
626 \\
718 \\
830\end{array}$ & $\begin{array}{l}1462 \\
1464 \\
1466 \\
1468 \\
1470\end{array}$ & $\begin{array}{l}32.8 \\
32.9 \\
32.9 \\
32.9 \\
33.0\end{array}$ & $\begin{array}{l}581 \\
675 \\
769 \\
864 \\
958\end{array}$ & $\begin{array}{l}1462 \\
1464 \\
1466 \\
1468 \\
1470\end{array}$ & $\begin{array}{l}5 \\
6 \\
6\end{array}$ & $\begin{array}{l}972 \\
076 \\
182 \\
287 \\
391\end{array}$ & $\begin{array}{l}1462 \\
1464 \\
1466 \\
1468 \\
1470\end{array}$ & $\begin{array}{l}72 . \\
72 . \\
72 . \\
72 .\end{array}$ \\
\hline $\begin{array}{l}0.253866 \\
0.254251 \\
0.254638 \\
0.255022 \\
0.255409\end{array}$ & $\begin{array}{l}1472 \\
1474 \\
1476 \\
1478 \\
1480\end{array}$ & $\begin{array}{l}3.3 \\
3.3 \\
3.3 \\
3.3 \\
3.3\end{array}$ & $\begin{array}{l}954 \\
094 \\
248 \\
423 \\
611\end{array}$ & $\begin{array}{l}1472 \\
1474 \\
1476 \\
1478 \\
1480\end{array}$ & $\begin{array}{l}33.0 \\
33.1 \\
33.1 \\
33.2 \\
33.2\end{array}$ & & $\begin{array}{l}1472 \\
1474 \\
1476 \\
1478 \\
1480\end{array}$ & 7 & $\begin{array}{l}496 \\
601 \\
706 \\
811 \\
915\end{array}$ & $\begin{array}{l}1472 \\
1474 \\
1476 \\
1478 \\
1480\end{array}$ & $\begin{array}{l}72.6 \\
72.7 \\
72.8 \\
72.9 \\
73.0\end{array}$ \\
\hline $\begin{array}{l}0.255796 \\
0.256181 \\
0,256569 \\
0.256954 \\
0.257340\end{array}$ & $\begin{array}{l}1482 \\
1484 \\
1486 \\
1488 \\
1490\end{array}$ & $\begin{array}{l}3.3 \\
3.3 \\
3.3 \\
3.3 \\
3.3\end{array}$ & $\begin{array}{l}3.223814 \\
3.232037 \\
3.240273 \\
3.248523 \\
3.256795\end{array}$ & $\begin{array}{l}1482 \\
1484 \\
1486 \\
1488 \\
1490\end{array}$ & $\begin{array}{l}33.3 \\
33.3 \\
33.3 \\
33.4 \\
33.4\end{array}$ & $\begin{array}{l}0.091525 \\
0.091621 \\
0.091715 \\
0.091811 \\
0.091907\end{array}$ & $\begin{array}{l}1482 \\
1484 \\
1486 \\
1488 \\
1490\end{array}$ & $\begin{array}{l}81.8 \\
81.9 \\
81.9 \\
82.0 \\
82.0\end{array}$ & $\begin{array}{l}0.075021 \\
0.075126 \\
0.075230 \\
0.075335 \\
0.075440\end{array}$ & $\begin{array}{l}1482 \\
1484 \\
1486 \\
1488 \\
1490\end{array}$ & $\begin{array}{l}73.1 \\
73.2 \\
73.3 \\
73.4 \\
73.5\end{array}$ \\
\hline $\begin{array}{l}0.257728 \\
0.258113 \\
0.258500 \\
0.258887 \\
0.259274\end{array}$ & $\begin{array}{l}1492 \\
1494 \\
1496 \\
1498 \\
1500\end{array}$ & $\begin{array}{l}3.3 \\
3.3 \\
3.3 \\
3.3 \\
3.3\end{array}$ & $\begin{array}{l}3.265079 \\
3.273379 \\
3.281698 \\
3.290029 \\
3.298375\end{array}$ & $\begin{array}{l}1492 \\
1494 \\
1496 \\
1498 \\
1500\end{array}$ & $\begin{array}{l}33.5 \\
33.5 \\
33.6 \\
33.6 \\
33.7\end{array}$ & $\begin{array}{l}0.092002 \\
0.092098 \\
0.092194 \\
0.092290 \\
0.092386\end{array}$ & $\begin{array}{l}1492 \\
1494 \\
1496 \\
1498 \\
1500\end{array}$ & $\begin{array}{l}82.0 \\
82.1 \\
82.1 \\
82.2 \\
82.2\end{array}$ & $\begin{array}{l}0.075546 \\
0.075651 \\
0.075755 \\
0.075861 \\
0.075966\end{array}$ & $\begin{array}{l}1492 \\
1494 \\
1496 \\
1498 \\
1500\end{array}$ & $\begin{array}{l}73 \\
73 \\
73 \\
73\end{array}$ \\
\hline
\end{tabular}


Geologic age, in millions of years, calculated from the atomic ratios of indicated isotopes

\begin{tabular}{|c|c|c|c|c|c|c|c|c|c|c|c|}
\hline \multicolumn{3}{|c|}{$N_{206} / N_{238}$} & \multicolumn{3}{|c|}{$N_{207} / N_{235}$} & \multicolumn{3}{|c|}{$N_{207} / N_{206}$} & \multicolumn{3}{|c|}{$N_{208} / N_{232}$} \\
\hline \multirow[b]{2}{*}{ Ratio } & \multicolumn{2}{|c|}{ Age } & \multirow[b]{2}{*}{ Ratio } & \multicolumn{2}{|c|}{ Age } & \multirow[b]{2}{*}{ Ratio } & \multicolumn{2}{|c|}{ Age } & \multirow[b]{2}{*}{ Ratio } & \multicolumn{2}{|c|}{ Age } \\
\hline & \begin{tabular}{|c|}
$\begin{array}{c}\text { Number } \\
\text { of } \\
\text { years }\end{array}$ \\
\end{tabular} & $\begin{array}{c}\text { Error } \\
\pm\end{array}$ & & \begin{tabular}{|c|}
$\begin{array}{c}\text { Number } \\
\text { of } \\
\text { years }\end{array}$ \\
\end{tabular} & $\begin{array}{c}\text { Error } \\
\pm\end{array}$ & & $\begin{array}{c}\text { Number } \\
\text { of } \\
\text { years }\end{array}$ & $\begin{array}{c}\text { Error } \\
\pm \\
\end{array}$ & & $\begin{array}{c}\text { Number } \\
\text { of } \\
\text { years }\end{array}$ & $\begin{array}{c}\text { Error } \\
\pm\end{array}$ \\
\hline $\begin{array}{l}0.260243 \\
0.261211 \\
0.262180 \\
0.263151 \\
0.264120\end{array}$ & $\begin{array}{l}1505 \\
1510 \\
1515 \\
1520 \\
1525\end{array}$ & $\begin{array}{l}3.3 \\
3.3 \\
3.4 \\
3.4 \\
3.4\end{array}$ & $\begin{array}{l}3.319322 \\
3.340369 \\
3.361520 \\
3.382772 \\
3.404125\end{array}$ & $\begin{array}{l}1505 \\
1510 \\
1515 \\
1520 \\
1525\end{array}$ & $\begin{array}{l}33.9 \\
34.0 \\
34.1 \\
34.2\end{array}$ & $\begin{array}{l}626 \\
868 \\
111 \\
354 \\
3598\end{array}$ & $\begin{array}{l}1505 \\
1510 \\
1515 \\
1520 \\
1525\end{array}$ & $\begin{array}{l}82.3 \\
82.4 \\
82.5 \\
82.6 \\
82.7\end{array}$ & $\begin{array}{l}0.076229 \\
0.076491 \\
0.076754 \\
0.077017 \\
0.077279\end{array}$ & $\begin{array}{l}1505 \\
1510 \\
1515 \\
1520 \\
1525\end{array}$ & $\begin{array}{l}74.2 \\
74.4 \\
74.7 \\
74.9 \\
75.2\end{array}$ \\
\hline $\begin{array}{l}0.2650_{94} \\
0.2660_{67} \\
0.2670_{39} \\
0.2680_{13} \\
0.268989\end{array}$ & $\begin{array}{l}1530 \\
1535 \\
1540 \\
1545 \\
1550\end{array}$ & $\begin{array}{l}3.4 \\
3.4 \\
3.4 \\
3.4 \\
3.4\end{array}$ & $\begin{array}{l}3.425585 \\
3.447149 \\
3.468821 \\
3.490599 \\
3.512473\end{array}$ & $\begin{array}{l}1530 \\
1535 \\
1540 \\
1545 \\
1550\end{array}$ & $\begin{array}{l}34.3 \\
34.4 \\
34.6 \\
34.7 \\
34.8\end{array}$ & $\begin{array}{l}842 \\
088 \\
335 \\
582 \\
829\end{array}$ & $\begin{array}{l}1530 \\
1535 \\
1540 \\
1545 \\
1550\end{array}$ & $\begin{array}{l}82.8 \\
82.9 \\
83.0 \\
83.1 \\
83.2\end{array}$ & $\begin{array}{l}542 \\
806 \\
068 \\
331 \\
595\end{array}$ & $\begin{array}{l}1530 \\
1535 \\
1540 \\
1545 \\
1550\end{array}$ & $\begin{array}{l}75.4 \\
75.7 \\
75.9 \\
76.2 \\
76.4\end{array}$ \\
\hline $\begin{array}{l}0.269964 \\
0.270941 \\
0.271917 \\
0.272895 \\
0.273872\end{array}$ & $\begin{array}{l}1555 \\
1560 \\
1565 \\
1570 \\
1575\end{array}$ & $\begin{array}{l}3.4 \\
3.5 \\
3.5 \\
3.5 \\
3.5\end{array}$ & $\begin{array}{l}7463 \\
6557 \\
3761 \\
1073 \\
3489\end{array}$ & $\begin{array}{l}1555 \\
1560 \\
1565 \\
1570 \\
1575\end{array}$ & $\begin{array}{l}34.9 \\
35.0 \\
35.1 \\
35.2 \\
35.3\end{array}$ & $\begin{array}{l}78 \\
28 \\
378 \\
330 \\
82\end{array}$ & $\begin{array}{l}1555 \\
1560 \\
1565 \\
1570 \\
1575\end{array}$ & $\begin{array}{l}83.4 \\
83.5 \\
83.6 \\
83.7\end{array}$ & $\begin{array}{l}57 \\
21 \\
84 \\
49 \\
12\end{array}$ & $\begin{array}{l}1555 \\
1560 \\
1565 \\
1570 \\
1575\end{array}$ & $\begin{array}{l}76.7 \\
76.9 \\
77.1 \\
77.4 \\
77.6\end{array}$ \\
\hline $\begin{array}{l}0.274852 \\
0.275833 \\
0.276814 \\
0.277796 \\
0.278777\end{array}$ & $\begin{array}{l}1580 \\
1585 \\
1590 \\
1595 \\
1600\end{array}$ & $\begin{array}{l}3.5 \\
3.5 \\
3.5 \\
3.5 \\
3.5\end{array}$ & $\begin{array}{l}19 \\
56 \\
06 \\
069 \\
35\end{array}$ & $\begin{array}{l}1580 \\
1585 \\
1590 \\
1595 \\
1600\end{array}$ & $\begin{array}{l}35.5 \\
35.6 \\
35.7 \\
35.8 \\
35.9\end{array}$ & $\begin{array}{l}35 \\
88 \\
43 \\
98 \\
55\end{array}$ & $\begin{array}{l}1580 \\
1585 \\
1590 \\
1595 \\
1600\end{array}$ & $\begin{array}{l}83 \\
84 \\
84\end{array}$ & $\begin{array}{l}76 \\
39 \\
02 \\
66 \\
30\end{array}$ & $\begin{array}{l}1580 \\
1585 \\
1590 \\
1595 \\
1600\end{array}$ & $\begin{array}{l}77.9 \\
78.1 \\
78.4 \\
78.6 \\
78.9\end{array}$ \\
\hline $\begin{array}{l}0.279761 \\
0.280745 \\
0.281729 \\
0.282714 \\
0.283702\end{array}$ & $\begin{array}{l}1605 \\
1610 \\
1615 \\
1620 \\
1625\end{array}$ & $\begin{array}{l}3.6 \\
3.6 \\
3.6 \\
3.6 \\
3.6\end{array}$ & $\begin{array}{l}3.76 \\
3.78 \\
3.85 \\
3.8 ? \\
3.85\end{array}$ & $\begin{array}{l}1605 \\
1610 \\
1615 \\
1620 \\
1625\end{array}$ & $\begin{array}{l}36.0 \\
36.1 \\
36.2 \\
36.4 \\
36.5\end{array}$ & $\begin{array}{l}612 \\
870 \\
128 \\
388 \\
648\end{array}$ & $\begin{array}{l}1605 \\
1610 \\
1615 \\
1620 \\
1625\end{array}$ & $\begin{array}{l}84 \\
8 \\
84 \\
8\end{array}$ & & $\begin{array}{l}1605 \\
1610 \\
1615 \\
1620 \\
1625\end{array}$ & $\begin{array}{l}79.1 \\
79.4 \\
79.6 \\
79.9 \\
80.1\end{array}$ \\
\hline $\begin{array}{l}0.284688 \\
0.285676 \\
0.286663 \\
0.287653 \\
0.288642\end{array}$ & $\begin{array}{l}1630 \\
1635 \\
1640 \\
1645 \\
1650\end{array}$ & $\begin{array}{l}3.6 \\
3.6 \\
3.6 \\
3.6 \\
3.7\end{array}$ & $\begin{array}{l}430 \\
198 \\
082 \\
079 \\
190\end{array}$ & $\begin{array}{l}1630 \\
1635 \\
1640 \\
1645 \\
1650\end{array}$ & $\begin{array}{l}36 \\
36 \\
36 \\
36 \\
37\end{array}$ & $\begin{array}{l}10 \\
172 \\
35 \\
99 \\
64\end{array}$ & $\begin{array}{l}1630 \\
1635 \\
1640 \\
1645 \\
1650\end{array}$ & & $\begin{array}{l}14 \\
78 \\
43 \\
08 \\
73\end{array}$ & $\begin{array}{l}1630 \\
1635 \\
1640 \\
1645 \\
1650\end{array}$ & $\begin{array}{l}80.4 \\
80.6 \\
80.8 \\
81.1 \\
81.3\end{array}$ \\
\hline $\begin{array}{l}289632 \\
290624 \\
291616 \\
292610\end{array}$ & $\begin{array}{l}1655 \\
1660 \\
1665 \\
1670 \\
1675\end{array}$ & $\begin{array}{l}3.7 \\
3.7 \\
3.7 \\
3.7 \\
3.7\end{array}$ & $\begin{array}{l}424 \\
775 \\
244 \\
834 \\
538\end{array}$ & $\begin{array}{l}1655 \\
1660 \\
1665 \\
1670\end{array}$ & $\begin{array}{l}37.1 \\
37.3 \\
37.4 \\
37.5 \\
37.6\end{array}$ & $\begin{array}{l}30 \\
96 \\
64 \\
32 \\
01\end{array}$ & $\begin{array}{l}1655 \\
1660 \\
1665 \\
1670 \\
1675\end{array}$ & $\begin{array}{l}8 \\
8 \\
8 \\
8\end{array}$ & & $\begin{array}{l}1655 \\
1660 \\
1665 \\
1670 \\
1675\end{array}$ & $\begin{array}{l}82.1 \\
82.3 \\
82.6\end{array}$ \\
\hline $\begin{array}{l}294598 \\
0.295592 \\
296589 \\
0.297584 \\
0.298584\end{array}$ & $\begin{array}{l}1680 \\
1685 \\
1690 \\
1695 \\
1700\end{array}$ & $\begin{array}{l}3.7 \\
3.7 \\
3.7 \\
3.8 \\
3.8\end{array}$ & $\begin{array}{l}4.120370 \\
4.145318 \\
4.170392 \\
4.195587 \\
4.220898\end{array}$ & $\begin{array}{l}1680 \\
1685 \\
1690 \\
1695 \\
1700\end{array}$ & $\begin{array}{l}37 \\
37 \\
37 \\
38 \\
38\end{array}$ & $\begin{array}{l}71 \\
43 \\
14 \\
88 \\
60\end{array}$ & $\begin{array}{l}1680 \\
1685 \\
1690 \\
1695 \\
1700\end{array}$ & $\begin{array}{l}85.7 \\
85.8 \\
85.9\end{array}$ & $\begin{array}{l}60 \\
25 \\
90 \\
55 \\
21\end{array}$ & $\begin{array}{l}1680 \\
1685 \\
1690 \\
1695 \\
1700\end{array}$ & $\begin{array}{l}82.8 \\
83.1 \\
83.3 \\
83.6 \\
83.8\end{array}$ \\
\hline $\begin{array}{l}0.299581 \\
0.300580 \\
0.301581 \\
0.302580 \\
0.303581\end{array}$ & $\begin{array}{l}1705 \\
1710 \\
1715 \\
1720 \\
1725\end{array}$ & $\begin{array}{l}3.8 \\
3.8 \\
3.8 \\
3.8 \\
3.8\end{array}$ & $\begin{array}{l}4.246342 \\
4.271905 \\
4.297593 \\
4.323407 \\
4.349342\end{array}$ & $\begin{array}{l}1705 \\
1710 \\
1715 \\
1720 \\
1725\end{array}$ & $\begin{array}{l}38.3 \\
38.4 \\
38.5 \\
38.6 \\
38.7\end{array}$ & $\begin{array}{l}2935 \\
3211 \\
3487 \\
3765 \\
4043\end{array}$ & $\begin{array}{l}1705 \\
1710 \\
1715 \\
1720 \\
1725\end{array}$ & $\begin{array}{l}86.2 \\
86.3 \\
86.4 \\
86.5\end{array}$ & $\begin{array}{l}86 \\
51 \\
17 \\
83 \\
49\end{array}$ & $\begin{array}{l}1705 \\
1710 \\
1715 \\
1720 \\
1725\end{array}$ & $\begin{array}{l}84 \\
84 \\
84 \\
84 \\
85\end{array}$ \\
\hline $\begin{array}{r}304584 \\
305588 \\
306591 \\
307595 \\
308599\end{array}$ & $\begin{array}{l}1730 \\
1735 \\
1740 \\
1745 \\
1750\end{array}$ & $\begin{array}{l}3.8 \\
3.8 \\
3.9 \\
3.9 \\
3.9\end{array}$ & $\begin{array}{l}4.375408 \\
4.401602 \\
4.427922 \\
4.454374 \\
4.480945\end{array}$ & $\begin{array}{l}1730 \\
1735 \\
1740 \\
1745 \\
1750\end{array}$ & $\begin{array}{l}38.8 \\
38.9 \\
39.0 \\
39.2 \\
39.3\end{array}$ & $\begin{array}{l}0.104322 \\
0.104602 \\
0.104883 \\
0.105165 \\
0.105448\end{array}$ & $\begin{array}{l}1730 \\
1735 \\
1740 \\
1745 \\
1750\end{array}$ & $\begin{array}{l}86.7 \\
86.8 \\
86.9 \\
87.0 \\
87.1\end{array}$ & $\begin{array}{l}0.088114 \\
0.088380 \\
0.088645 \\
0.088910 \\
0.089176\end{array}$ & $\begin{array}{l}1730 \\
1735 \\
1740 \\
1745 \\
1750\end{array}$ & $\begin{array}{l}85.3 \\
85.5 \\
85.8 \\
86.0 \\
86.3\end{array}$ \\
\hline
\end{tabular}


Geologic age, in millions of years, calculated from the atomic ratios of indicated isotopes

\begin{tabular}{|c|c|c|c|c|c|c|c|c|c|c|c|}
\hline \multicolumn{3}{|c|}{$N_{206} / N_{238}$} & \multicolumn{3}{|c|}{$N_{207} / N_{235}$} & \multicolumn{3}{|c|}{$N_{207} / N_{206}$} & \multicolumn{3}{|c|}{$N_{208} / N_{232}$} \\
\hline \multirow[b]{2}{*}{ Ratio } & \multicolumn{2}{|c|}{ Age } & \multirow[b]{2}{*}{ Ratio } & \multicolumn{2}{|c|}{ Age } & \multirow[b]{2}{*}{ Ratio } & \multicolumn{2}{|c|}{ Age } & \multirow[b]{2}{*}{ Ratio } & \multicolumn{2}{|c|}{ Age } \\
\hline & \begin{tabular}{|c|}
$\begin{array}{c}\text { Number } \\
\text { of } \\
\text { years }\end{array}$ \\
\end{tabular} & $\begin{array}{c}\text { Error } \\
\pm\end{array}$ & & $\begin{array}{c}\text { Number } \\
\text { of } \\
\text { years }\end{array}$ & $\begin{array}{c}\text { Error } \\
\pm\end{array}$ & & \begin{tabular}{|c|} 
Number \\
of \\
years
\end{tabular} & $\begin{array}{c}\text { Error } \\
\pm\end{array}$ & & \begin{tabular}{|c|}
$\begin{array}{c}\text { Number } \\
\text { of } \\
\text { years }\end{array}$ \\
\end{tabular} & $\begin{array}{c}\text { Error } \\
\pm\end{array}$ \\
\hline $\begin{array}{l}0.309606 \\
0.310614 \\
0.311621 \\
0.312630 \\
0.313637\end{array}$ & $\begin{array}{l}1755 \\
1760 \\
1765 \\
1770 \\
1775\end{array}$ & $\begin{array}{l}3.9 \\
3.9 \\
3.9 \\
3.9 \\
3.9\end{array}$ & $\begin{array}{l}4.507653 \\
4.534493 \\
4.561462 \\
4.588554 \\
4.615787\end{array}$ & $\begin{array}{l}1755 \\
1760 \\
1765 \\
1770 \\
1775\end{array}$ & $\begin{array}{l}39.4 \\
39.5 \\
39.6 \\
39.7 \\
39.8\end{array}$ & $\begin{array}{l}0.105732 \\
0.106016 \\
0.106302 \\
0.106588 \\
0.106877\end{array}$ & $\begin{array}{l}1755 \\
1760 \\
1765 \\
1770 \\
1775\end{array}$ & $\begin{array}{l}87.2 \\
87.3 \\
87.4 \\
87.5 \\
87.6\end{array}$ & $\begin{array}{l}0.089442 \\
0.089708 \\
0.089974 \\
0.090240 \\
0.090506\end{array}$ & $\begin{array}{l}1755 \\
1760 \\
1765 \\
1770 \\
1775\end{array}$ & $\begin{array}{l}86.5 \\
86.8 \\
87.0 \\
87.3 \\
87.5\end{array}$ \\
\hline $\begin{array}{l}0.314648 \\
0.315658 \\
0.316671 \\
0.317682 \\
0.318696\end{array}$ & $\begin{array}{l}1780 \\
1785 \\
1790 \\
1795 \\
1800\end{array}$ & $\begin{array}{l}3.9 \\
4.0 \\
4.0 \\
4.0 \\
4.0\end{array}$ & $\begin{array}{l}4.643153 \\
4.670650 \\
4.698282 \\
4.726043 \\
4.753945\end{array}$ & $\begin{array}{l}1780 \\
1785 \\
1790 \\
1795 \\
1800\end{array}$ & $\begin{array}{l}39.9 \\
40.1 \\
40.2 \\
40.3 \\
40.4\end{array}$ & $\begin{array}{l}0.107165 \\
0.107454 \\
0.107744 \\
0.108036 \\
0.108328\end{array}$ & $\begin{array}{l}1780 \\
1785 \\
1790 \\
1795 \\
1800\end{array}$ & $\begin{array}{l}87.7 \\
87.8 \\
87.9 \\
88.0 \\
88.1\end{array}$ & $\begin{array}{l}0.090772 \\
0.091038 \\
0.091305 \\
0.091572 \\
0.091839\end{array}$ & $\begin{array}{l}1780 \\
1785 \\
1790 \\
1795 \\
1800\end{array}$ & $\begin{array}{l}87.7 \\
88.0 \\
88.2 \\
88.5 \\
88.7\end{array}$ \\
\hline $\begin{array}{l}0.319708 \\
0.320723 \\
0.321739 \\
0.322756 \\
0.323771\end{array}$ & $\begin{array}{l}1805 \\
1810 \\
1815 \\
1820 \\
1825\end{array}$ & $\begin{array}{l}4.0 \\
4.0 \\
4.0 \\
4.0 \\
4.0\end{array}$ & $\begin{array}{l}4.781984 \\
4.810156 \\
4.838468 \\
4.866913 \\
4.895498\end{array}$ & $\begin{array}{l}1805 \\
1810 \\
1815 \\
1820 \\
1825\end{array}$ & $\begin{array}{l}40.5 \\
40.6 \\
40.7 \\
40.8 \\
41.0\end{array}$ & $\begin{array}{l}0.108622 \\
0.108916 \\
0.109211 \\
0.109507 \\
0.109805\end{array}$ & $\begin{array}{l}1805 \\
1810 \\
1815 \\
1820 \\
1825\end{array}$ & $\begin{array}{l}88.2 \\
88.3 \\
88.4 \\
88.5 \\
88.6\end{array}$ & $\begin{array}{l}0.092104 \\
0.092371 \\
0.092637 \\
0.092904 \\
0.093170\end{array}$ & $\begin{array}{l}1805 \\
1 \& 10 \\
1 \& 15 \\
1 \& ? 0 \\
1 \& ? 5\end{array}$ & $\begin{array}{l}89.0 \\
89.2 \\
89.5 \\
89.7 \\
90.0\end{array}$ \\
\hline $\begin{array}{l}0.324789 \\
0.325807 \\
0.326827 \\
0.327848 \\
0.328867\end{array}$ & $\begin{array}{l}1830 \\
1835 \\
1840 \\
1845 \\
1850\end{array}$ & $\begin{array}{l}4.1 \\
4.1 \\
4.1 \\
4.1 \\
4.1\end{array}$ & $\begin{array}{l}4.924230 \\
4.953097 \\
4.982106 \\
5.011250 \\
5.040542\end{array}$ & $\begin{array}{l}1830 \\
1835 \\
1840 \\
1845 \\
1850\end{array}$ & $\begin{array}{l}41.1 \\
41.2 \\
41.3 \\
41.4 \\
41.5\end{array}$ & $\begin{array}{l}0.110103 \\
0.110403 \\
0.110703 \\
0.111004 \\
0.111307\end{array}$ & $\begin{array}{l}1830 \\
1835 \\
1840 \\
1845 \\
1850\end{array}$ & $\begin{array}{l}88.7 \\
88.8 \\
88.9 \\
89.0 \\
89.1\end{array}$ & $\begin{array}{l}0.093437 \\
0.093704 \\
0.093971 \\
0.094238 \\
0.094505\end{array}$ & $\begin{array}{l}1 \& 30 \\
1 \& 35 \\
1 \varepsilon 40 \\
1 \& 45 \\
1 \varepsilon 50\end{array}$ & $\begin{array}{l}90.2 \\
90.5 \\
90.7 \\
91.0 \\
91.2\end{array}$ \\
\hline $\begin{array}{l}0.329890 \\
0.330911 \\
0.331935 \\
0.332959 \\
0.333984\end{array}$ & $\begin{array}{l}1855 \\
1860 \\
1865 \\
1870 \\
1875\end{array}$ & $\begin{array}{l}4.1 \\
4.1 \\
4.1 \\
4.1 \\
4.2\end{array}$ & $\begin{array}{l}5.069976 \\
5.099554 \\
5.129275 \\
5.159136 \\
5.189152\end{array}$ & $\begin{array}{l}1855 \\
1860 \\
1865 \\
1870 \\
1875\end{array}$ & $\begin{array}{l}41.6 \\
41.7 \\
41.9 \\
42.0 \\
42.1\end{array}$ & $\begin{array}{l}0.111609 \\
0.111914 \\
0.112219 \\
0.112525 \\
0.112833\end{array}$ & $\begin{array}{l}1855 \\
1860 \\
1865 \\
1870 \\
1875\end{array}$ & $\begin{array}{l}89.2 \\
89.3 \\
89.4 \\
89.5 \\
89.6\end{array}$ & $\begin{array}{l}772 \\
039 \\
306 \\
575 \\
842\end{array}$ & $\begin{array}{l}1 \varepsilon 55 \\
1 \varepsilon 60 \\
1 \varepsilon 55 \\
1 \varepsilon 70 \\
1 \varepsilon 75\end{array}$ & $\begin{array}{l}91.4 \\
91.7 \\
91.9 \\
92.2 \\
92.4\end{array}$ \\
\hline $\begin{array}{l}0.335009 \\
0.336035 \\
0.337062 \\
0.338089 \\
0.339119\end{array}$ & $\begin{array}{l}1880 \\
1885 \\
1890 \\
1895 \\
1900\end{array}$ & $\begin{array}{l}4.2 \\
4.2 \\
4.2 \\
4.2 \\
4.2\end{array}$ & $\begin{array}{l}5.219307 \\
5.249613 \\
5.280068 \\
5.310664 \\
5.341414\end{array}$ & $\begin{array}{l}1880 \\
1885 \\
1890 \\
1895 \\
1900\end{array}$ & $\begin{array}{l}42.2 \\
42.3 \\
42.4 \\
42.5 \\
42.6\end{array}$ & $\begin{array}{l}141 \\
451 \\
761 \\
073 \\
385\end{array}$ & $\begin{array}{l}1880 \\
1885 \\
1890 \\
1895 \\
1900\end{array}$ & $\begin{array}{l}89.7 \\
89.8 \\
89.9 \\
90.0 \\
90.1\end{array}$ & $\begin{array}{l}0.096109 \\
0.096377 \\
0.096644 \\
0.096913 \\
0.097180\end{array}$ & $\begin{array}{l}1880 \\
1895 \\
1890 \\
1895 \\
1900\end{array}$ & $\begin{array}{l}92.7 \\
92.9 \\
93.2 \\
93.4 \\
93.7\end{array}$ \\
\hline $\begin{array}{l}0.340147 \\
0.341179 \\
0.342209 \\
0.343241 \\
0.344274\end{array}$ & $\begin{array}{l}1905 \\
1910 \\
1915 \\
1920 \\
1925\end{array}$ & $\begin{array}{l}4.2 \\
4.2 \\
4.2 \\
4.3 \\
4.3\end{array}$ & $\begin{array}{l}5.372315 \\
5.403365 \\
5.434568 \\
5.465915 \\
5.497425\end{array}$ & $\begin{array}{l}1905 \\
1910 \\
1915 \\
1920 \\
1925\end{array}$ & $\begin{array}{l}42.7 \\
42.9 \\
43.0 \\
43.1 \\
43.2\end{array}$ & $\begin{array}{l}0.114699 \\
0.115013 \\
0.115329 \\
0.115645 \\
0.115963\end{array}$ & $\begin{array}{l}1905 \\
1910 \\
1915 \\
1920 \\
1925\end{array}$ & $\begin{array}{l}90.2 \\
90.3 \\
90.4 \\
90.5 \\
90.6\end{array}$ & $\begin{array}{l}0,097448 \\
0,097717 \\
0,097984 \\
0,098252 \\
0,098520\end{array}$ & $\begin{array}{l}1905 \\
1910 \\
1915 \\
1920 \\
1925\end{array}$ & $\begin{array}{l}93.9 \\
94.2 \\
94.4 \\
94.6 \\
94.9\end{array}$ \\
\hline $\begin{array}{l}0.345308 \\
0.346341 \\
0.347377 \\
0.348411 \\
0.349450\end{array}$ & $\begin{array}{l}1930 \\
1935 \\
1940 \\
1945 \\
1950\end{array}$ & $\begin{array}{l}4.3 \\
4.3 \\
4.3 \\
4.3 \\
4.3\end{array}$ & $\begin{array}{l}5.529084 \\
5.560898 \\
5.592872 \\
5.624987 \\
5.657274\end{array}$ & $\begin{array}{l}1930 \\
1935 \\
1940 \\
1945 \\
1950\end{array}$ & $\begin{array}{l}43.3 \\
43.4 \\
43.5 \\
43.6 \\
43.8\end{array}$ & $\begin{array}{l}0.116282 \\
0.116602 \\
0.116922 \\
0.117245 \\
0.117567\end{array}$ & $\begin{array}{l}1930 \\
1935 \\
1940 \\
1945 \\
1950\end{array}$ & $\begin{array}{l}90.7 \\
90.8 \\
90.9 \\
91.0 \\
91.1\end{array}$ & $\begin{array}{l}0.098788 \\
0.099056 \\
0.099324 \\
0.099593 \\
0.099862\end{array}$ & $\begin{array}{l}1930 \\
1935 \\
1940 \\
1945 \\
1950\end{array}$ & $\begin{array}{l}95.1 \\
95.4 \\
95.6 \\
95.9 \\
96.1\end{array}$ \\
\hline $\begin{array}{l}0.350488 \\
0.351525 \\
0.352564 \\
0.353604 \\
0.354645\end{array}$ & $\begin{array}{l}1955 \\
1960 \\
1965 \\
1970 \\
1975\end{array}$ & $\begin{array}{l}4.3 \\
4.3 \\
4.4 \\
4.4 \\
4.4\end{array}$ & $\begin{array}{l}5.689710 \\
5.722310 \\
5.755066 \\
5.787976 \\
5.8210_{53}\end{array}$ & $\begin{array}{l}1955 \\
1960 \\
1965 \\
1970 \\
1975\end{array}$ & $\begin{array}{l}43.9 \\
44.0 \\
44.1 \\
44.2 \\
44.3\end{array}$ & $\begin{array}{l}0.117891 \\
0.118217 \\
0.118543 \\
0.118870 \\
0.119199\end{array}$ & $\begin{array}{l}1955 \\
1960 \\
1965 \\
1970 \\
1975\end{array}$ & $\begin{array}{l}91.2 \\
91.3 \\
91.4 \\
91.5 \\
91.6\end{array}$ & $\begin{array}{l}0.100130 \\
0.100398 \\
0.100668 \\
0.100936 \\
0.101205\end{array}$ & $\begin{array}{l}1955 \\
1960 \\
1965 \\
1970 \\
1975\end{array}$ & $\begin{array}{l}96.4 \\
96.6 \\
96.9 \\
97.1 \\
97.4\end{array}$ \\
\hline $\begin{array}{l}0.355685 \\
0.356728 \\
0.357771 \\
0.358816 \\
0.359859\end{array}$ & $\begin{array}{l}1980 \\
1985 \\
1990 \\
1995 \\
2000\end{array}$ & $\begin{array}{l}4.4 \\
4.4 \\
4.4 \\
4.4 \\
4.4\end{array}$ & $\begin{array}{l}5.854291 \\
5.887691 \\
5.921252 \\
5.954970 \\
5.988862\end{array}$ & $\begin{array}{l}1980 \\
1985 \\
1990 \\
1995 \\
2000\end{array}$ & $\begin{array}{l}44.4 \\
44.5 \\
44.7 \\
44.8 \\
44.9\end{array}$ & $\begin{array}{l}0.119529 \\
0.119859 \\
0.120191 \\
0.120524 \\
0.120858\end{array}$ & $\begin{array}{l}1980 \\
1985 \\
1990 \\
1995 \\
2000\end{array}$ & $\begin{array}{l}91.7 \\
91.8 \\
91.9 \\
92.0 \\
92.1\end{array}$ & $\begin{array}{l}0.101473 \\
0.101742 \\
0.102010 \\
0.102279 \\
0.102549\end{array}$ & $\begin{array}{l}1980 \\
1985 \\
1990 \\
1995 \\
2000\end{array}$ & $\begin{array}{l}97.6 \\
97.9 \\
98.1 \\
98.3 \\
98.6\end{array}$ \\
\hline
\end{tabular}


Geologic age, in millions of years, calculated from the atomic ratios of indicated isotopes

\begin{tabular}{|c|c|c|c|c|c|c|c|c|c|c|c|}
\hline \multicolumn{3}{|c|}{$N_{206} / N_{238}$} & \multicolumn{3}{|c|}{$N_{207} / N_{235}$} & \multicolumn{3}{|c|}{$N_{207} / N_{206}$} & \multicolumn{3}{|c|}{$N_{208} / N_{232}$} \\
\hline \multirow[b]{2}{*}{ Ratio } & \multicolumn{2}{|c|}{ Age } & \multirow[b]{2}{*}{ Ratio } & \multicolumn{2}{|c|}{ Age } & \multirow[b]{2}{*}{ Ratio } & \multicolumn{2}{|c|}{ Age } & \multirow[b]{2}{*}{ Ratio } & \multicolumn{2}{|c|}{ Age } \\
\hline & $\begin{array}{c}\begin{array}{c}\text { Number } \\
\text { of } \\
\text { years }\end{array} \\
\end{array}$ & $\begin{array}{c}\text { Error } \\
\pm\end{array}$ & & \begin{tabular}{|c|}
$\begin{array}{c}\text { Number } \\
\text { of } \\
\text { years }\end{array}$ \\
\end{tabular} & $\begin{array}{c}\text { Error } \\
\pm\end{array}$ & & \begin{tabular}{|c|}
$\begin{array}{c}\text { Number } \\
\text { of } \\
\text { years }\end{array}$ \\
\end{tabular} & $\begin{array}{c}\text { Error } \\
\pm\end{array}$ & & \begin{tabular}{|c|}
$\begin{array}{c}\text { Number } \\
\text { of } \\
\text { years }\end{array}$ \\
\end{tabular} & $\begin{array}{c}\text { Error } \\
\pm\end{array}$ \\
\hline $\begin{array}{l}0.360905 \\
0.361951 \\
0.362998 \\
0.364047 \\
0.365094\end{array}$ & $\begin{array}{l}2005 \\
2010 \\
2015 \\
2020 \\
2025\end{array}$ & $\begin{array}{l}4.4 \\
4.5 \\
4.5 \\
4.5 \\
4.5\end{array}$ & $\begin{array}{l}6.022918 \\
6.057140 \\
6.091526 \\
6.126076 \\
6.160799\end{array}$ & $\begin{array}{l}2005 \\
2010 \\
2015 \\
2020 \\
2025\end{array}$ & $\begin{array}{l}45.0 \\
45.1 \\
45.2 \\
45.3 \\
45.4\end{array}$ & $\begin{array}{l}0.121193 \\
0.121530 \\
0.121867 \\
0.122205 \\
0.122545\end{array}$ & $\begin{array}{l}2005 \\
2010 \\
2015 \\
2020 \\
2025\end{array}$ & $\begin{array}{l}92.2 \\
92.3 \\
92.4 \\
92.5 \\
92.6\end{array}$ & $\begin{array}{l}0.102818 \\
0.103087 \\
0.103356 \\
0.103626 \\
0.103895\end{array}$ & $\begin{array}{l}2005 \\
2010 \\
2015 \\
2020 \\
2025\end{array}$ & $\begin{array}{l}98.8 \\
99.1 \\
99.3 \\
99.6 \\
99.8\end{array}$ \\
\hline $\begin{array}{l}0.366144 \\
0.367194 \\
0.368245 \\
0.369296 \\
0.370349\end{array}$ & $\begin{array}{l}2030 \\
2035 \\
2040 \\
2045 \\
2050\end{array}$ & $\begin{array}{l}4.5 \\
4.5 \\
4.5 \\
4.5 \\
4.5\end{array}$ & $\begin{array}{l}6.195695 \\
6.230758 \\
6.265992 \\
6.301391 \\
6.336972\end{array}$ & $\begin{array}{l}2030 \\
2035 \\
2040 \\
2045 \\
2050\end{array}$ & $\begin{array}{l}45.6 \\
45.7 \\
45.8 \\
45.9 \\
46.0\end{array}$ & $\begin{array}{l}0.122886 \\
0.123228 \\
0.123571 \\
0.123916 \\
0.124261\end{array}$ & $\begin{array}{l}2030 \\
2035 \\
2040 \\
2.045 \\
2050\end{array}$ & $\begin{array}{l}92.7 \\
92.8 \\
92.9 \\
93.0 \\
93.1\end{array}$ & $\begin{array}{l}166 \\
435 \\
704 \\
974 \\
243\end{array}$ & $\begin{array}{l}2030 \\
2035 \\
2040 \\
2045 \\
2050\end{array}$ & $\begin{array}{l}100.1 \\
100.3 \\
1006 \\
1008 \\
101.1\end{array}$ \\
\hline $\begin{array}{l}0.371403 \\
0.372458 \\
0.373511 \\
0.374569 \\
0.375624\end{array}$ & $\begin{array}{l}2055 \\
2060 \\
2065 \\
2070 \\
2075\end{array}$ & $\begin{array}{l}4.6 \\
4.6 \\
4.6 \\
4.6 \\
4.6\end{array}$ & $\begin{array}{l}6.372720 \\
6.408648 \\
6.444749 \\
6.481019 \\
6.517473\end{array}$ & $\begin{array}{l}2055 \\
2060 \\
2065 \\
2070 \\
2075\end{array}$ & $\begin{array}{l}46.1 \\
46.2 \\
46.3 \\
46.5 \\
46.6\end{array}$ & $\begin{array}{l}0.124607 \\
0.124955 \\
0.125305 \\
0.125654 \\
0.126006\end{array}$ & $\begin{array}{l}2055 \\
2060 \\
2065 \\
2070 \\
2075\end{array}$ & $\begin{array}{l}93.2 \\
93.3 \\
93.4 \\
93.5 \\
93.6\end{array}$ & $\begin{array}{l}0.105514 \\
0.105783 \\
0.106053 \\
0.106322 \\
0.106593\end{array}$ & $\begin{array}{l}2055 \\
2060 \\
2065 \\
2070 \\
2075\end{array}$ & $\begin{array}{l}101.3 \\
101.5 \\
101.8 \\
102.0 \\
102.3\end{array}$ \\
\hline $\begin{array}{l}376682 \\
377742 \\
378799 \\
379859 \\
380921\end{array}$ & $\begin{array}{l}2080 \\
2085 \\
2090 \\
2095 \\
2100\end{array}$ & $\begin{array}{l}4.6 \\
4.6 \\
4.6 \\
4.6 \\
4.7\end{array}$ & $\begin{array}{l}6.554104 \\
6.590912 \\
6.627904 \\
6.665064 \\
6.702414\end{array}$ & $\begin{array}{l}2080 \\
2085 \\
2090 \\
2095 \\
2100\end{array}$ & $\begin{array}{l}46.7 \\
46.8 \\
46.9 \\
47.0 \\
47.1\end{array}$ & $\begin{array}{l}0.126358 \\
0.126711 \\
0.127067 \\
0.127423 \\
0.127779\end{array}$ & $\begin{array}{l}2080 \\
2085 \\
2090 \\
2095 \\
2100\end{array}$ & $\begin{array}{l}93.7 \\
93.8 \\
93.9 \\
94.0 \\
94.1\end{array}$ & $\begin{array}{l}863 \\
133 \\
402 \\
674 \\
944\end{array}$ & $\begin{array}{l}2080 \\
2085 \\
2090 \\
2095 \\
2100\end{array}$ & $\begin{array}{l}102.5 \\
102.8 \\
103.0 \\
103.3 \\
103.5\end{array}$ \\
\hline $\begin{array}{l}0.381982 \\
0.383043 \\
0.384109 \\
0.385172 \\
0.386236\end{array}$ & $\begin{array}{l}2105 \\
2110 \\
2115 \\
2120 \\
2125\end{array}$ & $\begin{array}{l}4.7 \\
4.7 \\
4.7 \\
4.7 \\
4.7\end{array}$ & $\begin{array}{l}6.739946 \\
6.777662 \\
6.815561 \\
6.853638 \\
6.891908\end{array}$ & $\begin{array}{l}2105 \\
2110 \\
2115 \\
2120 \\
2125\end{array}$ & $\begin{array}{l}47.2 \\
47.3 \\
47.5 \\
47.6 \\
47.7\end{array}$ & $\begin{array}{l}0.128138 \\
0.128498 \\
0.128858 \\
0.129220 \\
0.129584\end{array}$ & $\begin{array}{l}2105 \\
2110 \\
2115 \\
2120 \\
2125\end{array}$ & $\begin{array}{l}94.2 \\
94.3 \\
94.4 \\
94.5 \\
94.6\end{array}$ & $\begin{array}{l}0.108214 \\
0.108486 \\
0.108756 \\
0.109026 \\
0.109296\end{array}$ & $\begin{array}{l}2105 \\
2110 \\
2115 \\
2120 \\
2125\end{array}$ & $\begin{array}{l}103.8 \\
104.0 \\
104.3 \\
104.5 \\
104.8\end{array}$ \\
\hline $\begin{array}{l}0.387303 \\
0.388368 \\
0.389436 \\
0.390504 \\
0.391573\end{array}$ & $\begin{array}{l}2130 \\
2135 \\
2140 \\
2145 \\
2150\end{array}$ & $\begin{array}{l}4.7 \\
4.7 \\
4.7 \\
4.8 \\
4.8\end{array}$ & $\begin{array}{l}6.930364 \\
6.969005 \\
7.007839 \\
7.046852 \\
7.086061\end{array}$ & $\begin{array}{l}2130 \\
2135 \\
2140 \\
2145 \\
2150\end{array}$ & $\begin{array}{l}47.8 \\
47.9 \\
48.0 \\
48.1 \\
48.2\end{array}$ & $\begin{array}{l}0.129948 \\
0.130314 \\
0.130681 \\
0.131049 \\
0.131419\end{array}$ & $\begin{array}{l}2130 \\
2135 \\
2140 \\
2145 \\
2150\end{array}$ & $\begin{array}{l}94.7 \\
94.8 \\
94.9 \\
95.0 \\
95.1\end{array}$ & $\begin{array}{l}0.109568 \\
0.109838 \\
0.110108 \\
0.110380 \\
0.110652\end{array}$ & $\begin{array}{l}2130 \\
2135 \\
2140 \\
2145 \\
2150\end{array}$ & $\begin{array}{l}105.0 \\
105.2 \\
105.5 \\
105.7 \\
106.0\end{array}$ \\
\hline $\begin{array}{l}0.392643 \\
0.393714 \\
0.394783 \\
0.395857 \\
0.396930\end{array}$ & $\begin{array}{l}2155 \\
2160 \\
2165 \\
2170 \\
2175\end{array}$ & $\begin{array}{l}4.8 \\
4.8 \\
4.8 \\
4.8 \\
4.8\end{array}$ & $\begin{array}{l}7.125465 \\
7.165058 \\
7.204844 \\
7.244819 \\
7.284996\end{array}$ & $\begin{array}{l}2155 \\
2160 \\
2165 \\
2170 \\
2175\end{array}$ & $\begin{array}{l}48.4 \\
48.5 \\
48.6 \\
48.7 \\
48.8\end{array}$ & $\begin{array}{l}0.131789 \\
0.132161 \\
0.132535 \\
0.132909 \\
0.133285\end{array}$ & $\begin{array}{l}2155 \\
2160 \\
2165 \\
2170 \\
2175\end{array}$ & $\begin{array}{l}95.2 \\
95.3 \\
95.5 \\
95.6 \\
95.7\end{array}$ & $\begin{array}{l}922 \\
94 \\
965 \\
38 \\
08\end{array}$ & $\begin{array}{l}2155 \\
2160 \\
2165 \\
2170 \\
2175\end{array}$ & $\begin{array}{l}106.2 \\
106.5 \\
106.7 \\
107.0 \\
107.2\end{array}$ \\
\hline $\begin{array}{l}0.398005 \\
0.399078 \\
0.400154 \\
0.401231 \\
0.402307\end{array}$ & $\begin{array}{l}2180 \\
2185 \\
2190 \\
2195 \\
2200\end{array}$ & $\begin{array}{l}4.8 \\
4.8 \\
4.9 \\
4.9 \\
4.9\end{array}$ & $\begin{array}{l}7.325365 \\
7.365933 \\
7.406698 \\
7.447655 \\
7.488819\end{array}$ & $\begin{array}{l}2180 \\
2185 \\
2190 \\
2195 \\
2200\end{array}$ & $\begin{array}{l}48.9 \\
49.0 \\
49.1 \\
49.3 \\
49.4\end{array}$ & $\begin{array}{l}0.133661 \\
0.134040 \\
0.134419 \\
0.134800 \\
0.135182\end{array}$ & $\begin{array}{l}2180 \\
2185 \\
2190 \\
2195 \\
2200\end{array}$ & $\begin{array}{l}95.8 \\
95.9 \\
96.0\end{array}$ & $\begin{array}{l}0.112279 \\
0.112551 \\
0.112822 \\
0.113095 \\
0.113366\end{array}$ & $\begin{array}{l}2180 \\
2185 \\
2190 \\
2195 \\
2200\end{array}$ & $\begin{array}{l}107.5 \\
107.7 \\
108.0 \\
108.2 \\
108.5\end{array}$ \\
\hline $\begin{array}{l}0.403387 \\
0.404464 \\
0.405546 \\
0.406625 \\
0.407706\end{array}$ & $\begin{array}{l}2205 \\
2210 \\
2215 \\
2220 \\
2225\end{array}$ & $\begin{array}{l}4.9 \\
4.9 \\
4.9 \\
4.9 \\
4.9\end{array}$ & $\begin{array}{l}7.530185 \\
7.571751 \\
7.613518 \\
7.655485 \\
7.697660\end{array}$ & $\begin{array}{l}2205 \\
2210 \\
2215 \\
2220 \\
2225\end{array}$ & $\begin{array}{l}49.5 \\
49.6 \\
49.7 \\
49.8 \\
49.9\end{array}$ & $\begin{array}{l}0.135565 \\
0.135951 \\
0.136336 \\
0.136723 \\
0.137112\end{array}$ & $\begin{array}{l}2205 \\
2210 \\
2215 \\
2220 \\
2225\end{array}$ & $\begin{array}{l}96.3 \\
96.4 \\
96.5 \\
96.6\end{array}$ & $\begin{array}{l}0.113637 \\
0.113909 \\
0.114181 \\
0.114453 \\
0.114724\end{array}$ & $\begin{array}{l}2205 \\
2210 \\
2215 \\
2220 \\
2225\end{array}$ & $\begin{array}{l}108.7 \\
108.9 \\
109.2 \\
109.4 \\
109.7\end{array}$ \\
\hline $\begin{array}{l}0.408788 \\
0.409873 \\
0.410956 \\
0.412041 \\
0.413125\end{array}$ & $\begin{array}{l}2230 \\
2235 \\
2240 \\
2245 \\
2250\end{array}$ & $\begin{array}{l}4.9 \\
5.0 \\
5.0 \\
5.0 \\
50\end{array}$ & $\begin{array}{l}7.740041 \\
7.782628 \\
7.825426 \\
7.868422 \\
7.911637\end{array}$ & $\begin{array}{l}2230 \\
2235 \\
2240 \\
2245 \\
2250\end{array}$ & $\begin{array}{l}50.0 \\
50.2 \\
50.3 \\
50.4 \\
50.5\end{array}$ & $\begin{array}{l}0.137502 \\
0.137893 \\
0.138286 \\
0.138679 \\
0.139075\end{array}$ & $\begin{array}{l}2230 \\
2235 \\
2240 \\
2245 \\
2250\end{array}$ & $\begin{array}{l}96.8 \\
96.9 \\
97.0 \\
97.1 \\
97.2\end{array}$ & $\begin{array}{l}0.114996 \\
0.115269 \\
0.115540 \\
0.115814 \\
0.116087\end{array}$ & $\begin{array}{l}2230 \\
2235 \\
2240 \\
2245 \\
2250\end{array}$ & $\begin{array}{l}009.9 \\
1110.2 \\
110.4 \\
110.7 \\
110.9\end{array}$ \\
\hline
\end{tabular}


Geologic age, in millions of years, calculated from the atomic ratios of indicated isotopes

\begin{tabular}{|c|c|c|c|c|c|c|c|c|c|c|c|}
\hline \multicolumn{3}{|c|}{$N_{206} / N_{238}$} & \multicolumn{3}{|c|}{$N_{207} / N_{235}$} & \multicolumn{3}{|c|}{$N_{207} / N_{206}$} & \multicolumn{3}{|c|}{$N_{208} / N_{232}$} \\
\hline \multirow[b]{2}{*}{ Ratio } & \multicolumn{2}{|c|}{ Age } & \multirow[b]{2}{*}{ Ratio } & \multicolumn{2}{|c|}{ Age } & \multirow[b]{2}{*}{ Ratio } & \multicolumn{2}{|c|}{ Age } & \multirow[b]{2}{*}{ Ratio } & \multicolumn{2}{|c|}{ Age } \\
\hline & \begin{tabular}{|c|}
$\begin{array}{c}\text { Number } \\
\text { of } \\
\text { years }\end{array}$ \\
\end{tabular} & $\begin{array}{c}\text { Error } \\
\pm\end{array}$ & & $\begin{array}{c}\text { Number } \\
\text { of } \\
\text { years }\end{array}$ & $\begin{array}{c}\text { Error } \\
\pm\end{array}$ & & $\begin{array}{c}\text { Number } \\
\text { of } \\
\text { years }\end{array}$ & $\begin{array}{c}\text { Error } \\
\pm \\
\end{array}$ & & \begin{tabular}{|c|} 
Number \\
of \\
years
\end{tabular} \mid & $\begin{array}{c}\text { Error } \\
\pm\end{array}$ \\
\hline $\begin{array}{l}0.414211 \\
0.415300 \\
0.416386 \\
0.417477 \\
0.418565\end{array}$ & $\begin{array}{l}2255 \\
2260 \\
2265 \\
2270 \\
2275\end{array}$ & $\begin{array}{l}5.0 \\
5.0 \\
5.0 \\
5.0 \\
5.0\end{array}$ & $\begin{array}{l}7.95506 \\
7.99870 \\
8.04254 \\
8.08660 \\
8.13088\end{array}$ & $\begin{array}{l}2255 \\
2260 \\
2265 \\
2270 \\
2275\end{array}$ & $\begin{array}{l}50.6 \\
50.7 \\
50.8 \\
50.9 \\
51.1\end{array}$ & $\begin{array}{l}0.139472 \\
0.139869 \\
0.140269 \\
0.140669 \\
0.141071\end{array}$ & $\begin{array}{l}2255 \\
2260 \\
2265 \\
2270 \\
2275\end{array}$ & $\begin{array}{l}97.3 \\
97.4 \\
97.5 \\
97.6 \\
97.7\end{array}$ & $\begin{array}{l}0.116360 \\
0.116632 \\
0.116904 \\
0.117176 \\
0.117450\end{array}$ & $\begin{array}{l}2255 \\
2260 \\
2265 \\
2270 \\
2275\end{array}$ & $\begin{array}{l}111.2 \\
111.4 \\
111.7 \\
11.19 \\
112.1\end{array}$ \\
\hline $\begin{array}{l}0.419658 \\
0.420747 \\
0.421840 \\
0.422932 \\
0.424026\end{array}$ & $\begin{array}{l}2280 \\
2285 \\
2290 \\
2295 \\
2300\end{array}$ & $\begin{array}{l}5.1 \\
5.1 \\
5.1 \\
5.1 \\
5.1\end{array}$ & $\begin{array}{l}8.17537 \\
8.22008 \\
8.26501 \\
8.310_{14} \\
8.35551\end{array}$ & $\begin{array}{l}2280 \\
2285 \\
2290 \\
2295 \\
2300\end{array}$ & $\begin{array}{l}51.2 \\
51.3 \\
51.4 \\
51.5 \\
51.6\end{array}$ & $\begin{array}{l}0.141474 \\
0.141879 \\
0.142285 \\
0.142693 \\
0.143102\end{array}$ & $\begin{array}{l}2280 \\
2285 \\
2290 \\
2295 \\
2300\end{array}$ & $\begin{array}{l}97.8 \\
97.9 \\
98.0 \\
98.1 \\
98.2\end{array}$ & $\begin{array}{l}0.117722 \\
0.117995 \\
0.118267 \\
0.118540 \\
0.118813\end{array}$ & $\begin{array}{l}2280 \\
2285 \\
2290 \\
2295 \\
2300\end{array}$ & $\begin{array}{l}112.4 \\
112.6 \\
112.9 \\
113.1 \\
113.4\end{array}$ \\
\hline $\begin{array}{l}0.425121 \\
0.426217 \\
0.427313 \\
0.428409 \\
0.429509\end{array}$ & $\begin{array}{l}2305 \\
2310 \\
2315 \\
2320 \\
2325\end{array}$ & $\begin{array}{l}5.1 \\
5.1 \\
5.1 \\
5.1 \\
5.2\end{array}$ & $\begin{array}{l}8.40110 \\
8.44691 \\
8.49294 \\
8.53919 \\
8.58567\end{array}$ & $\begin{array}{l}2305 \\
2310 \\
2315 \\
2320 \\
2325\end{array}$ & $\begin{array}{l}51.7 \\
51.8 \\
51.9 \\
52.1 \\
52.2\end{array}$ & $\begin{array}{l}0.143512 \\
0.143923 \\
0.144337 \\
0.144751 \\
0.145167\end{array}$ & $\begin{array}{l}2305 \\
2310 \\
2315 \\
2320 \\
2325\end{array}$ & $\begin{array}{l}98.3 \\
98.4 \\
98.5 \\
98.6 \\
98.7\end{array}$ & $\begin{array}{l}086 \\
359 \\
632 \\
907 \\
180\end{array}$ & $\begin{array}{l}2305 \\
2310 \\
2315 \\
2320 \\
2325\end{array}$ & $\begin{array}{l}113.6 \\
113.9 \\
114.1 \\
114.4 \\
114.6\end{array}$ \\
\hline $\begin{array}{l}0.430608 \\
0.431707 \\
0.432807 \\
0.433910 \\
0.435012\end{array}$ & $\begin{array}{l}2330 \\
2335 \\
2340 \\
2345 \\
2350\end{array}$ & $\begin{array}{l}5.2 \\
5.2 \\
5.2 \\
5.2 \\
5.2\end{array}$ & $\begin{array}{l}8.63238 \\
8.67932 \\
8.72649 \\
8.77387 \\
8.82150\end{array}$ & $\begin{array}{l}2330 \\
2335 \\
2340 \\
2345 \\
2350\end{array}$ & $\begin{array}{l}52.3 \\
52.4 \\
52.5 \\
52.6 \\
52.7\end{array}$ & $\begin{array}{l}0.145584 \\
0.146003 \\
0.146423 \\
0.146844 \\
0.147267\end{array}$ & $\begin{array}{l}2330 \\
2335 \\
2340 \\
2345 \\
2350\end{array}$ & $\begin{array}{l}98.8 \\
98.9 \\
99.0 \\
99.1 \\
99.3\end{array}$ & $\begin{array}{l}0.120453 \\
0.120727 \\
0.121001 \\
0.121274 \\
0.121548\end{array}$ & $\begin{array}{l}2330 \\
2335 \\
2340 \\
2345 \\
2350\end{array}$ & $\begin{array}{l}114.9 \\
115.1 \\
115.4 \\
115.6 \\
115.8\end{array}$ \\
\hline $\begin{array}{l}0.436116 \\
0.437219 \\
0.438325 \\
0.439430 \\
0.440537\end{array}$ & $\begin{array}{l}2355 \\
2360 \\
2365 \\
2370 \\
2375\end{array}$ & $\begin{array}{l}5.2 \\
5.2 \\
5.2 \\
5.3 \\
5.3\end{array}$ & $\begin{array}{l}8.86936 \\
8.91745 \\
8.96577 \\
9.01433 \\
9.06312\end{array}$ & $\begin{array}{l}2355 \\
2360 \\
2365 \\
2370 \\
2375\end{array}$ & $\begin{array}{l}52.8 \\
53.0 \\
53.1 \\
53.2 \\
53.3\end{array}$ & $\begin{array}{l}0.147691 \\
0.148118 \\
0.148544 \\
0.148973 \\
0.149403\end{array}$ & $\begin{array}{l}2355 \\
2360 \\
2365 \\
2370 \\
2375\end{array}$ & $\begin{array}{l}99.3 \\
99.5 \\
99.6 \\
99.7 \\
99.8\end{array}$ & $\begin{array}{l}822 \\
095 \\
369 \\
643 \\
916\end{array}$ & $\begin{array}{l}2355 \\
2360 \\
2365 \\
2370 \\
2375\end{array}$ & $\begin{array}{l}116.1 \\
116.3 \\
116.6 \\
116.8 \\
117.1\end{array}$ \\
\hline $\begin{array}{l}0.441643 \\
0.442752 \\
0.443862 \\
0.444971 \\
0.446082\end{array}$ & $\begin{array}{l}2380 \\
2385 \\
2390 \\
2395 \\
2400\end{array}$ & $\begin{array}{l}5.3 \\
5.3 \\
5.3 \\
5.3 \\
5.3\end{array}$ & $\begin{array}{l}9.11216 \\
9.16143 \\
9.21095 \\
9.26070 \\
9.31069\end{array}$ & $\begin{array}{l}2380 \\
2385 \\
2390 \\
2395 \\
2400\end{array}$ & $\begin{array}{l}53.4 \\
53.5 \\
53.6 \\
53.7 \\
53.9\end{array}$ & $\begin{array}{l}0.149835 \\
0.150268 \\
0.150703 \\
0.151139 \\
0.151577\end{array}$ & $\begin{array}{l}2380 \\
2385 \\
2390 \\
2395 \\
2400\end{array}$ & $\begin{array}{r}99.9 \\
100.0 \\
100.1 \\
100.2 \\
100.3\end{array}$ & $\begin{array}{l}3191 \\
3464 \\
3739 \\
4014 \\
4288\end{array}$ & $\begin{array}{l}2380 \\
2385 \\
2390 \\
2395 \\
2400\end{array}$ & $\begin{array}{l}117.3 \\
117.6 \\
117.8 \\
118.1 \\
118.3\end{array}$ \\
\hline $\begin{array}{l}0.447193 \\
0.448307 \\
0.449419 \\
0.450535 \\
0.451648\end{array}$ & $\begin{array}{l}2405 \\
2410 \\
2415 \\
2420 \\
2425\end{array}$ & $\begin{array}{l}5.3 \\
5.3 \\
5.4 \\
5.4 \\
5.4\end{array}$ & $\begin{array}{l}9.36093 \\
9.41142 \\
9.46216 \\
9.51313 \\
9.56436\end{array}$ & $\begin{array}{l}2405 \\
2410 \\
2415 \\
2420 \\
2425\end{array}$ & $\begin{array}{l}54.0 \\
54.1 \\
54.2 \\
54.3 \\
54.4\end{array}$ & $\begin{array}{l}0.1520_{16} \\
0.152456 \\
0.152899 \\
0.153341 \\
0.153787\end{array}$ & $\begin{array}{l}2405 \\
2410 \\
2415 \\
2420 \\
2425\end{array}$ & $\begin{array}{l}100.4 \\
100.5 \\
100.6 \\
100.7 \\
100.8\end{array}$ & $\begin{array}{l}562 \\
837 \\
837 \\
113 \\
387 \\
661\end{array}$ & $\begin{array}{l}2405 \\
2410 \\
2415 \\
2420 \\
2425\end{array}$ & $\begin{array}{l}118.6 \\
118.8 \\
119.0 \\
119.3 \\
119.5\end{array}$ \\
\hline $\begin{array}{l}0.452765 \\
0.453882 \\
0.454998 \\
0.456117 \\
0.457236\end{array}$ & $\begin{array}{l}2430 \\
2435 \\
2440 \\
2445 \\
2450\end{array}$ & $\begin{array}{l}5.4 \\
5.4 \\
5.4 \\
5.4 \\
5.4\end{array}$ & $\begin{array}{l}9.61584 \\
9.66756 \\
9.71955 \\
9.77177 \\
9.82426\end{array}$ & $\begin{array}{l}2430 \\
2435 \\
2440 \\
2445 \\
2450\end{array}$ & $\begin{array}{l}54.5 \\
54.6 \\
54.8 \\
54.9 \\
55.0\end{array}$ & $\begin{array}{l}0.154234 \\
0.154682 \\
0.155132 \\
0.155583 \\
0.156036\end{array}$ & $\begin{array}{l}2430 \\
2435 \\
2440 \\
2445 \\
2450\end{array}$ & $\begin{array}{l}100.9 \\
101.0 \\
101.1 \\
101.2 \\
101.3\end{array}$ & $\begin{array}{l}0.125936 \\
0.126210 \\
0,126485 \\
0.126760 \\
0.127036\end{array}$ & $\begin{array}{l}2430 \\
2435 \\
2440 \\
2445 \\
2450\end{array}$ & $\begin{array}{l}119.8 \\
120.0 \\
120.3 \\
120.5 \\
120.8\end{array}$ \\
\hline $\begin{array}{l}0.458357 \\
0.459479 \\
0.460602 \\
0.461723 \\
0.462849\end{array}$ & $\begin{array}{l}2455 \\
2460 \\
2465 \\
2470 \\
2475\end{array}$ & $\begin{array}{l}5.4 \\
5.5 \\
5.5 \\
5.5 \\
5.5\end{array}$ & $\begin{array}{r}9.87700 \\
9.93000 \\
9.98327 \\
10.03677 \\
10.09055\end{array}$ & $\begin{array}{l}2455 \\
2460 \\
2465 \\
2470 \\
2475\end{array}$ & $\begin{array}{l}55.1 \\
55.2 \\
55.3 \\
55.4 \\
55.5\end{array}$ & $\begin{array}{l}0.156490 \\
0.156945 \\
0.157403 \\
0.157862 \\
0.158322\end{array}$ & $\begin{array}{l}2455 \\
2460 \\
2465 \\
2470 \\
2475\end{array}$ & $\begin{array}{l}101.4 \\
101.5 \\
101.6 \\
101.8 \\
101.9\end{array}$ & $\begin{array}{l}0.127310 \\
0.127585 \\
0.127860 \\
0.128137 \\
0.128411\end{array}$ & $\begin{array}{l}2455 \\
2460 \\
2465 \\
2470 \\
2475\end{array}$ & $\begin{array}{l}121.0 \\
121.3 \\
121.5 \\
121.8 \\
122.0\end{array}$ \\
\hline $\begin{array}{l}463972 \\
.465097 \\
.466224 \\
4667352 \\
4668478\end{array}$ & $\begin{array}{l}2480 \\
2485 \\
2490 \\
2495 \\
2500\end{array}$ & $\begin{array}{l}5.5 \\
5.5 \\
5.5 \\
5.5 \\
5.5\end{array}$ & $\begin{array}{l}10.14460 \\
10.19890 \\
10.25347 \\
10.30830 \\
10.36340\end{array}$ & $\begin{array}{l}2480 \\
2485 \\
2490 \\
2495 \\
2500\end{array}$ & $\begin{array}{l}55.7 \\
55.8 \\
55.9 \\
56.0 \\
56.1\end{array}$ & $\begin{array}{l}0,158784 \\
0.159248 \\
0,159713 \\
0,160180 \\
0.160649\end{array}$ & $\begin{array}{l}2480 \\
2485 \\
2490 \\
2495 \\
2500\end{array}$ & $\begin{array}{l}102.0 \\
102.1 \\
102.2 \\
102.3 \\
102.4\end{array}$ & $\begin{array}{l}0.128687 \\
0.128963 \\
0.129239 \\
0.129515 \\
0.129790\end{array}$ & $\begin{array}{l}2480 \\
2485 \\
2490 \\
2495 \\
2.500\end{array}$ & $\begin{array}{l}122.3 \\
122.5 \\
122.7 \\
123.0 \\
123.2\end{array}$ \\
\hline
\end{tabular}


$2510-3000$

Geologic age, in millions of years, calculated from the atomic ratios of indicated isotopes

\begin{tabular}{|c|c|c|c|c|c|c|c|c|c|c|c|}
\hline \multicolumn{3}{|c|}{$N_{206} / N_{238}$} & \multicolumn{3}{|c|}{$N_{207} / N_{235}$} & \multicolumn{3}{|c|}{$N_{207} / N_{206}$} & \multicolumn{3}{|c|}{$N_{208} / N_{232}$} \\
\hline \multirow[b]{2}{*}{ Ratio } & \multicolumn{2}{|c|}{ Age } & \multirow[b]{2}{*}{ Ratio } & \multicolumn{2}{|c|}{ Age } & \multirow[b]{2}{*}{ Ratio } & \multicolumn{2}{|c|}{ Age } & \multirow[b]{2}{*}{ Ratio } & \multicolumn{2}{|c|}{ Age } \\
\hline & \begin{tabular}{|c|}
$\begin{array}{c}\text { Number } \\
\text { of } \\
\text { years }\end{array}$ \\
\end{tabular} & $\begin{array}{c}\text { Error } \\
\pm\end{array}$ & & \begin{tabular}{|c|}
$\begin{array}{c}\text { Number } \\
\text { of } \\
\text { years }\end{array}$ \\
\end{tabular} & $\begin{array}{c}\text { Error } \\
\pm\end{array}$ & & \begin{tabular}{|c|}
$\begin{array}{c}\text { Number } \\
\text { of } \\
\text { years }\end{array}$ \\
\end{tabular} & $\begin{array}{c}\text { Error } \\
\pm \\
\end{array}$ & & \begin{tabular}{|c|}
$\begin{array}{c}\text { Number } \\
\text { of } \\
\text { years }\end{array}$ \\
\end{tabular} & $\begin{array}{c}\text { Error } \\
\pm\end{array}$ \\
\hline $\begin{array}{l}0.470737 \\
0.472999 \\
0.475266 \\
0.477535 \\
0.479806\end{array}$ & $\begin{array}{l}2510 \\
2520 \\
2530 \\
2540 \\
2550\end{array}$ & $\begin{array}{l}5.6 \\
5.6 \\
5.6 \\
5.6 \\
5.7\end{array}$ & $\begin{array}{l}10.47442 \\
10.58650 \\
10.69970 \\
10.81399 \\
10.92940\end{array}$ & $\begin{array}{l}2510 \\
2520 \\
2530 \\
2540 \\
2550\end{array}$ & $\begin{array}{l}56.3 \\
56.5 \\
56.8 \\
57.0 \\
57.2\end{array}$ & $\begin{array}{l}591 \\
539 \\
493 \\
455 \\
423\end{array}$ & $\begin{array}{l}2510 \\
2520 \\
2530 \\
2540 \\
2550\end{array}$ & $\begin{array}{l}102.6 \\
102.8 \\
103.0 \\
103.2 \\
103.4\end{array}$ & $\begin{array}{l}341 \\
893 \\
444 \\
997 \\
550\end{array}$ & $\begin{array}{l}251 C \\
252 C \\
253 C \\
254 C \\
255 C\end{array}$ & $\begin{array}{l}123.7 \\
124.2 \\
124.7 \\
125.2 \\
125.7\end{array}$ \\
\hline $\begin{array}{l}0.482083 \\
0.484363 \\
0.486644 \\
0.488933 \\
0.491222\end{array}$ & $\begin{array}{l}2560 \\
2570 \\
2580 \\
2590 \\
2600\end{array}$ & $\begin{array}{l}5.7 \\
5.7 \\
5.7 \\
5.7 \\
5.8\end{array}$ & $\begin{array}{l}11.04594 \\
11.16361 \\
11.28244 \\
11.40243 \\
11.52358\end{array}$ & $\begin{array}{l}2560 \\
2570 \\
2580 \\
2590 \\
2600\end{array}$ & $\begin{array}{l}57.4 \\
57.7 \\
57.9 \\
58.1 \\
58.3\end{array}$ & $\begin{array}{l}0.166397 \\
0.167378 \\
0.168367 \\
0.169361 \\
0.170363\end{array}$ & $\begin{array}{l}2560 \\
2570 \\
2580 \\
2590 \\
2600\end{array}$ & $\begin{array}{l}10 \\
10 \\
10 \\
10 \\
10\end{array}$ & $\begin{array}{l}104 \\
657 \\
210 \\
763 \\
317\end{array}$ & $\begin{array}{l}256 C \\
257 C \\
258 C \\
259 C \\
2600\end{array}$ & $\begin{array}{l}126.2 \\
126.7 \\
127.2 \\
127.7 \\
128.2\end{array}$ \\
\hline $\begin{array}{l}0.493515 \\
0.495813 \\
0.498113 \\
0.500418 \\
0.502726\end{array}$ & $\begin{array}{l}2610 \\
2620 \\
2630 \\
2640 \\
2650\end{array}$ & $\begin{array}{l}5.8 \\
5.8 \\
5.8 \\
5.9 \\
5.9\end{array}$ & $\begin{array}{l}11.64593 \\
11.76947 \\
11.89421 \\
12.02019 \\
12.14737\end{array}$ & $\begin{array}{l}2610 \\
2620 \\
2630 \\
2640 \\
2650\end{array}$ & $\begin{array}{l}58.6 \\
58.8 \\
59.0 \\
59.2 \\
59.5\end{array}$ & $\begin{array}{l}0.171372 \\
0.172387 \\
0.173409 \\
0.174439 \\
0.175475\end{array}$ & $\begin{array}{l}2610 \\
2620 \\
2630 \\
2640 \\
2650\end{array}$ & $\begin{array}{l}104.7 \\
104.9 \\
105.1 \\
105.3 \\
105.5\end{array}$ & $\begin{array}{l}871 \\
428 \\
982 \\
537 \\
092\end{array}$ & $\begin{array}{l}2610 \\
2620 \\
2630 \\
2640 \\
2650\end{array}$ & $\begin{array}{l}128.7 \\
129.2 \\
129.6 \\
130.1 \\
130.6\end{array}$ \\
\hline $\begin{array}{l}0.505037 \\
0.507352 \\
0.509671 \\
0.511992 \\
0.514318\end{array}$ & $\begin{array}{l}2660 \\
2670 \\
2680 \\
2690 \\
2700\end{array}$ & $\begin{array}{l}5.9 \\
5.9 \\
5.9 \\
6.0 \\
6.0\end{array}$ & $\begin{array}{l}12.27581 \\
12.40550 \\
12.53646 \\
12.66870 \\
12.80222\end{array}$ & $\begin{array}{l}2660 \\
2670 \\
2680 \\
2690 \\
2700\end{array}$ & $\begin{array}{l}59.7 \\
59.9 \\
60.1 \\
60.4 \\
60.6\end{array}$ & $\begin{array}{l}6519 \\
7570 \\
8628 \\
9694 \\
0767\end{array}$ & $\begin{array}{l}2660 \\
2670 \\
2680 \\
2690 \\
2700\end{array}$ & $\begin{array}{l}105.7 \\
106.0 \\
106.2 \\
106.4 \\
106.6\end{array}$ & $\begin{array}{l}647 \\
203 \\
760 \\
316 \\
874\end{array}$ & $\begin{array}{l}2660 \\
2670 \\
2680 \\
2690 \\
2700\end{array}$ & $\begin{array}{l}131.1 \\
131.6 \\
132.1 \\
132.6 \\
133.1\end{array}$ \\
\hline $\begin{array}{l}0.516647 \\
0.518981 \\
0.521316 \\
0.523656 \\
0.526000\end{array}$ & $\begin{array}{l}2710 \\
2720 \\
2730 \\
2740 \\
2750\end{array}$ & $\begin{array}{l}6.0 \\
6.0 \\
6.1 \\
6.1 \\
6.1\end{array}$ & $\begin{array}{l}12.93 \\
13.07 \\
13.2 \\
13.32 \\
13.48\end{array}$ & $\begin{array}{l}2710 \\
2720 \\
2730 \\
2740 \\
2750\end{array}$ & $\begin{array}{l}60.8 \\
61.0 \\
61.3 \\
61.5 \\
61.7\end{array}$ & $\begin{array}{l}847 \\
935 \\
030 \\
133 \\
244\end{array}$ & $\begin{array}{l}2710 \\
2720 \\
2730 \\
2740 \\
2750\end{array}$ & $\begin{array}{l}10 \\
10 \\
10 \\
10 \\
10\end{array}$ & $\begin{array}{l}30 \\
88 \\
846 \\
03 \\
061\end{array}$ & $\begin{array}{l}2710 \\
2720 \\
2730 \\
2740 \\
2750\end{array}$ & $\begin{array}{l}133.6 \\
134.1 \\
134.6 \\
135.1 \\
135.6\end{array}$ \\
\hline $\begin{array}{l}0.528349 \\
0.530698 \\
0.533052 \\
0.535410 \\
0.537771\end{array}$ & $\begin{array}{l}2760 \\
2770 \\
2780 \\
2790 \\
2800\end{array}$ & $\begin{array}{l}6.1 \\
6.1 \\
6.2 \\
6.2 \\
6.2\end{array}$ & $\begin{array}{l}13.63125 \\
13.77417 \\
13.91851 \\
14.06426 \\
14.21141\end{array}$ & $\begin{array}{l}2760 \\
2770 \\
2780 \\
2790 \\
2800\end{array}$ & $\begin{array}{l}61.9 \\
62.2 \\
62.4 \\
62.6 \\
62.8\end{array}$ & $\begin{array}{l}7361 \\
3488 \\
9622 \\
3764 \\
913\end{array}$ & $\begin{array}{l}2760 \\
2770 \\
2780 \\
2790 \\
2800\end{array}$ & $\begin{array}{l}107.9 \\
108.1 \\
108.3 \\
108.5 \\
108.7\end{array}$ & $\begin{array}{l}4219 \\
4778 \\
5338 \\
5896 \\
6456\end{array}$ & $\begin{array}{l}2760 \\
2770 \\
2780 \\
2790 \\
2800\end{array}$ & $\begin{array}{l}136.1 \\
136.5 \\
137.0 \\
137.5 \\
138.0\end{array}$ \\
\hline $\begin{array}{l}0.540137 \\
0.542506 \\
0.544878 \\
0.547254 \\
0.549634\end{array}$ & $\begin{array}{l}2810 \\
2820 \\
2830 \\
2840 \\
2850\end{array}$ & $\begin{array}{l}6.2 \\
6.3 \\
6.3 \\
6.3 \\
6.3\end{array}$ & $\begin{array}{l}14.36002 \\
14.510_{06} \\
14.66159 \\
14.81459 \\
14.96908\end{array}$ & $\begin{array}{l}2810 \\
2820 \\
2830 \\
2840 \\
2850\end{array}$ & $\begin{array}{l}63.1 \\
63.3 \\
63.5 \\
63.7 \\
64.0\end{array}$ & $\begin{array}{l}071 \\
236 \\
410 \\
592 \\
782\end{array}$ & $\begin{array}{l}2810 \\
2820 \\
2830 \\
2840 \\
2850\end{array}$ & $\begin{array}{l}108 \\
10 \\
10 \\
10 \\
10\end{array}$ & $\begin{array}{l}16 \\
75 \\
36 \\
95 \\
57\end{array}$ & $\begin{array}{l}2810 \\
2820 \\
2830 \\
2840 \\
2850\end{array}$ & $\begin{array}{l}138.5 \\
139.0 \\
139.5 \\
140.0 \\
140.5\end{array}$ \\
\hline $\begin{array}{l}0.552017 \\
0.554406 \\
0.556796 \\
0.559190 \\
0.561588\end{array}$ & $\begin{array}{l}2860 \\
2870 \\
2880 \\
2890 \\
2900\end{array}$ & $\begin{array}{l}6.3 \\
6.4 \\
6.4 \\
6.4 \\
6.4\end{array}$ & $\begin{array}{l}15.12508 \\
15.28260 \\
15.44167 \\
15.60229 \\
15.76447\end{array}$ & $\begin{array}{l}2860 \\
2870 \\
2880 \\
2890 \\
2900\end{array}$ & $\begin{array}{l}64.2 \\
64.4 \\
64.6 \\
64.9 \\
65.1\end{array}$ & $\begin{array}{l}0.198980 \\
0.200186 \\
0.201402 \\
0.202626 \\
0.203858\end{array}$ & $\begin{array}{l}2860 \\
2870 \\
2880 \\
2890 \\
2900\end{array}$ & $\begin{array}{l}110.0 \\
110.2 \\
110.4 \\
110.6 \\
110.9\end{array}$ & $\begin{array}{l}0.149818 \\
0.150381 \\
0.150942 \\
0.151503 \\
0.152065\end{array}$ & $\begin{array}{l}2860 \\
2870 \\
2880 \\
2890 \\
2900\end{array}$ & $\begin{array}{l}141.0 \\
141.5 \\
142.0 \\
142.5 \\
143.0\end{array}$ \\
\hline $\begin{array}{l}0.563993 \\
0.566397 \\
0.568805 \\
0.571216 \\
0.573635\end{array}$ & $\begin{array}{l}2910 \\
2920 \\
2930 \\
2940 \\
2950\end{array}$ & $\begin{array}{l}6.5 \\
6.5 \\
6.5 \\
6.5 \\
6.5\end{array}$ & $\begin{array}{l}15.92826 \\
16.09361 \\
16.26061 \\
16.42923 \\
16.59949\end{array}$ & $\begin{array}{l}2910 \\
2920 \\
2930 \\
2940 \\
2950\end{array}$ & $\begin{array}{l}65.3 \\
65.5 \\
65.8 \\
66.0 \\
66.2\end{array}$ & $\begin{array}{l}0.205097 \\
0.206347 \\
0.207605 \\
0.208873 \\
0.210148\end{array}$ & $\begin{array}{l}2910 \\
2920 \\
2930 \\
2940 \\
2950\end{array}$ & $\begin{array}{l}111.1 \\
111.3 \\
111.5 \\
111.7 \\
111.9\end{array}$ & $\begin{array}{l}0.152627 \\
0.153192 \\
0.153754 \\
0.154317 \\
0.154880\end{array}$ & $\begin{array}{l}2910 \\
2920 \\
2930 \\
2940 \\
2950\end{array}$ & $\begin{array}{l}143.5 \\
143.9 \\
144.4 \\
144.9 \\
145.4\end{array}$ \\
\hline $\begin{array}{l}0.576055 \\
0.578479 \\
0.580907 \\
0.583339 \\
0.585775\end{array}$ & $\begin{array}{l}2960 \\
2970 \\
2980 \\
2990 \\
3000\end{array}$ & $\begin{array}{l}6.6 \\
6.6 \\
6.6 \\
6.6 \\
6.7\end{array}$ & $\begin{array}{l}16.77143 \\
16.94502 \\
17.12034 \\
17.29737 \\
17.47610\end{array}$ & $\begin{array}{l}2960 \\
2970 \\
2980 \\
2990 \\
3000\end{array}$ & $\begin{array}{l}66.4 \\
66.6 \\
66.9 \\
67.1 \\
67.3\end{array}$ & $\begin{array}{l}0.211432 \\
0.212726 \\
0.214028 \\
0.215340 \\
0.216660\end{array}$ & $\begin{array}{l}2960 \\
2970 \\
2980 \\
2990 \\
3000\end{array}$ & $\begin{array}{l}112.1 \\
112.4 \\
112.6 \\
112.8 \\
113.0\end{array}$ & $\begin{array}{l}0.155445 \\
0.156008 \\
0.156572 \\
0.157138 \\
0.157704\end{array}$ & $\begin{array}{l}2960 \\
2970 \\
2980 \\
2990 \\
3000\end{array}$ & $\begin{array}{l}145.9 \\
146.4 \\
146.9 \\
147.4 \\
147.9\end{array}$ \\
\hline
\end{tabular}


$3010-3500$

Geologic age, in millions of years, calculated from the atomic ratios of indicated isotopes

\begin{tabular}{|c|c|c|c|c|c|c|c|c|c|c|c|}
\hline \multicolumn{3}{|c|}{$N_{206} / N_{238}$} & \multicolumn{3}{|c|}{$N_{207} / N_{235}$} & \multicolumn{3}{|c|}{$N_{207} / N_{206}$} & \multicolumn{3}{|c|}{$N_{208} / N_{232}$} \\
\hline \multirow[b]{2}{*}{ Ratio } & \multicolumn{2}{|c|}{ Age } & \multirow[b]{2}{*}{ Ratio } & \multicolumn{2}{|c|}{ Age } & \multirow[b]{2}{*}{ Ratio } & \multicolumn{2}{|c|}{ Age } & \multirow[b]{2}{*}{ Ratio } & \multicolumn{2}{|c|}{ Age } \\
\hline & \begin{tabular}{|c|}
$\begin{array}{c}\text { Number } \\
\text { of } \\
\text { years }\end{array}$ \\
\end{tabular} & $\begin{array}{c}\text { Error } \\
\pm\end{array}$ & & \begin{tabular}{|c|}
$\begin{array}{c}\text { Number } \\
\text { of } \\
\text { years }\end{array}$ \\
\end{tabular} & $\begin{array}{c}\text { Error } \\
\pm\end{array}$ & & \begin{tabular}{|c|}
$\begin{array}{c}\text { Number } \\
\text { of } \\
\text { years }\end{array}$ \\
\end{tabular} & $\begin{array}{c}\text { Error } \\
\pm \\
\end{array}$ & & \begin{tabular}{|c|}
$\begin{array}{c}\text { Number } \\
\text { of } \\
\text { years }\end{array}$ \\
\end{tabular} & $\begin{array}{c}\text { Error } \\
\pm\end{array}$ \\
\hline $\begin{array}{l}0.588213 \\
0.590656 \\
0.593103 \\
0.595553 \\
0.598006\end{array}$ & $\begin{array}{l}3010 \\
3020 \\
3030 \\
3040 \\
3050\end{array}$ & $\begin{array}{l}6.7 \\
6.7 \\
6.7 \\
6.7 \\
6.8\end{array}$ & $\begin{array}{l}17.65661 \\
17.83885 \\
18.02289 \\
1820874 \\
18.39637\end{array}$ & $\begin{array}{l}3010 \\
3020 \\
3030 \\
3040 \\
3050\end{array}$ & $\begin{array}{l}67.5 \\
67.8 \\
68.0 \\
68.2 \\
68.4\end{array}$ & $\begin{array}{l}0.217991 \\
0.219330 \\
0.220678 \\
0.222037 \\
0.223404\end{array}$ & $\begin{array}{l}3010 \\
3020 \\
3030 \\
3040 \\
3050\end{array}$ & $\begin{array}{l}113.2 \\
113.4 \\
113.7 \\
113.9 \\
114.1\end{array}$ & $\begin{array}{l}0.158268 \\
0.158833 \\
0.159399 \\
0.159966 \\
0.160531\end{array}$ & $\begin{array}{l}301 C \\
302 C \\
3030 \\
304 C \\
305 C\end{array}$ & $\begin{array}{l}148.4 \\
148.9 \\
149.4 \\
149.9 \\
150.4\end{array}$ \\
\hline $\begin{array}{l}0.600464 \\
0.602928 \\
0.605393 \\
0.607862 \\
0.610335\end{array}$ & $\begin{array}{l}3060 \\
3070 \\
3080 \\
3090 \\
3100\end{array}$ & $\begin{array}{l}6.8 \\
6.8 \\
6.8 \\
6.9 \\
6.9\end{array}$ & $\begin{array}{l}18.58586 \\
18.77718 \\
18,97040 \\
19.16550 \\
19.36248\end{array}$ & $\begin{array}{l}3060 \\
3070 \\
3080 \\
3090 \\
3100\end{array}$ & $\begin{array}{l}68.7 \\
68.9 \\
69.1 \\
69.3 \\
69.6\end{array}$ & $\begin{array}{l}0.224782 \\
0.226168 \\
0.227564 \\
0.228971 \\
0.230387\end{array}$ & $\begin{array}{l}3060 \\
3070 \\
3080 \\
3090 \\
3100\end{array}$ & $\begin{array}{l}114.3 \\
114.5 \\
114.7 \\
115.0 \\
115.2\end{array}$ & $\begin{array}{l}0.161098 \\
0.161665 \\
0.162233 \\
0.162800 \\
0.163368\end{array}$ & $\begin{array}{l}306 C \\
307 C \\
308 C \\
309 C \\
310 C\end{array}$ & $\begin{array}{l}150.8 \\
151.3 \\
151.8 \\
152.3 \\
152.8\end{array}$ \\
\hline $\begin{array}{l}0.612811 \\
0.615292 \\
0.617777 \\
0.620266 \\
0.622757\end{array}$ & $\begin{array}{l}3110 \\
3120 \\
3130 \\
3140 \\
3150\end{array}$ & $\begin{array}{l}6.9 \\
6.9 \\
6.9 \\
7.0 \\
7.0\end{array}$ & $\begin{array}{l}19.5 \\
19.7 \\
19.9 \\
20.1 \\
20.3\end{array}$ & $\begin{array}{l}3110 \\
3120 \\
3130 \\
3140 \\
3150\end{array}$ & $\begin{array}{l}69.8 \\
70.0 \\
70.2 \\
70.5 \\
70.7\end{array}$ & $\begin{array}{l}814 \\
249 \\
696 \\
152 \\
619\end{array}$ & $\begin{array}{l}3110 \\
3120 \\
3130 \\
3140 \\
3150\end{array}$ & $\begin{array}{l}11 \\
11 \\
11 \\
11 \\
11\end{array}$ & $\begin{array}{l}936 \\
504 \\
072 \\
642 \\
211\end{array}$ & $\begin{array}{l}311 C \\
312 C \\
313 C \\
314 C \\
315 C\end{array}$ & $\begin{array}{l}153.3 \\
153.8 \\
154.3 \\
154.8 \\
155.3\end{array}$ \\
\hline $\begin{array}{l}0.625255 \\
0.627752 \\
0.630256 \\
0.632765 \\
0.635275\end{array}$ & $\begin{array}{l}3160 \\
3170 \\
3180 \\
3190 \\
3200\end{array}$ & $\begin{array}{l}7.0 \\
7.0 \\
7.1 \\
7.1 \\
7.1\end{array}$ & $\begin{array}{l}555 \\
641 \\
935 \\
437 \\
146\end{array}$ & $\begin{array}{l}3160 \\
3170 \\
3180 \\
3190 \\
3200\end{array}$ & $\begin{array}{l}70.9 \\
71.1 \\
71.4 \\
71.6 \\
71.8\end{array}$ & $\begin{array}{l}095 \\
583 \\
081 \\
589 \\
108\end{array}$ & $\begin{array}{l}3160 \\
3170 \\
3180 \\
3190 \\
3200\end{array}$ & $\begin{array}{l}11 \\
11 \\
11 \\
11 \\
11\end{array}$ & $\begin{array}{l}80 \\
49 \\
20 \\
90 \\
60\end{array}$ & $\begin{array}{l}316 C \\
317 C \\
318 C \\
319 C \\
320 C\end{array}$ & $\begin{array}{l}155.8 \\
156.3 \\
156.8 \\
157.3 \\
157.7\end{array}$ \\
\hline $\begin{array}{l}0.637791 \\
0.640310 \\
0.642833 \\
0.645360 \\
0.647891\end{array}$ & $\begin{array}{l}3210 \\
3220 \\
3230 \\
3240 \\
3250\end{array}$ & $\begin{array}{l}7.1 \\
7.1 \\
7.2 \\
7.2 \\
7.2\end{array}$ & $\begin{array}{l}21.66070 \\
21.88206 \\
22.10561 \\
22.33133 \\
22.55924\end{array}$ & $\begin{array}{l}3210 \\
3220 \\
3230 \\
3240 \\
3250\end{array}$ & $\begin{array}{l}72.0 \\
72.3 \\
72.5 \\
72.7 \\
72.9\end{array}$ & $\begin{array}{l}0.246638 \\
0.248178 \\
0.249729 \\
0.251292 \\
0.252865\end{array}$ & $\begin{array}{l}3210 \\
3220 \\
3230 \\
3240 \\
3250\end{array}$ & $\begin{array}{l}117.6 \\
117.8 \\
118.0 \\
118.2 \\
1184\end{array}$ & $\begin{array}{l}631 \\
203 \\
774 \\
346 \\
917\end{array}$ & $\begin{array}{l}3210 \\
3220 \\
3230 \\
3240 \\
3250\end{array}$ & $\begin{array}{l}158.2 \\
158.7 \\
159.2 \\
159.7 \\
160.2\end{array}$ \\
\hline $\begin{array}{l}0.650425 \\
0.652965 \\
0.655506 \\
0.658052 \\
0.660600\end{array}$ & $\begin{array}{l}3260 \\
3270 \\
3280 \\
3290 \\
3300\end{array}$ & $\begin{array}{l}7.2 \\
7.3 \\
7.3 \\
7.3 \\
7.3\end{array}$ & $\begin{array}{l}940 \\
179 \\
646 \\
344 \\
270\end{array}$ & $\begin{array}{l}3260 \\
3270 \\
3280 \\
3290 \\
3300\end{array}$ & $\begin{array}{l}73.2 \\
73.4 \\
73.6 \\
73.8 \\
74.1\end{array}$ & $\begin{array}{l}4449 \\
6044 \\
7651 \\
9270 \\
0900\end{array}$ & $\begin{array}{l}3260 \\
3270 \\
3280 \\
3290 \\
3300\end{array}$ & $\begin{array}{l}11 \\
11 \\
11 \\
11 \\
11\end{array}$ & $\begin{array}{l}89 \\
62 \\
35 \\
08 \\
82\end{array}$ & $\begin{array}{l}3260 \\
3270 \\
3280 \\
3290 \\
3300\end{array}$ & $\begin{array}{l}160.7 \\
161.2 \\
161.7 \\
162.2 \\
162.7\end{array}$ \\
\hline $\begin{array}{l}0.663155 \\
.665714 \\
.668276 \\
0670843 \\
0673412\end{array}$ & $\begin{array}{l}3310 \\
3320 \\
3330 \\
3340 \\
3350\end{array}$ & $\begin{array}{l}7.3 \\
7.4 \\
7.4 \\
7.4 \\
7.4\end{array}$ & $\begin{array}{l}23.97432 \\
24.21829 \\
24.46465 \\
24.71342 \\
24.96460\end{array}$ & $\begin{array}{l}3310 \\
3320 \\
3330 \\
3340 \\
3350\end{array}$ & $\begin{array}{l}74.3 \\
74.5 \\
74.7 \\
75.0 \\
75.2\end{array}$ & $\begin{array}{l}41 \\
93 \\
57 \\
33 \\
21\end{array}$ & $\begin{array}{l}3310 \\
3320 \\
3330 \\
3340 \\
3350\end{array}$ & $\begin{array}{l}8 \\
0 \\
2 \\
4 \\
6\end{array}$ & $\begin{array}{l}354 \\
928 \\
502 \\
077 \\
651\end{array}$ & $\begin{array}{l}3310 \\
3320 \\
3330 \\
3340 \\
3350\end{array}$ & $\begin{array}{l}163.2 \\
163.7 \\
164.2 \\
164.6 \\
165.1\end{array}$ \\
\hline $\begin{array}{l}0.675985 \\
0.678564 \\
0.681148 \\
0.683733 \\
0.686323\end{array}$ & $\begin{array}{l}3360 \\
3370 \\
3380 \\
3390 \\
3400\end{array}$ & $\begin{array}{l}7.5 \\
7.5 \\
7.5 \\
7.5 \\
7.5\end{array}$ & $\begin{array}{l}25.21826 \\
25.47438 \\
25.73301 \\
25.99418 \\
26.25787\end{array}$ & $\begin{array}{l}3360 \\
3370 \\
3380 \\
3390 \\
3400\end{array}$ & $\begin{array}{l}75.4 \\
75.6 \\
75.8 \\
76.1 \\
76.3\end{array}$ & $\begin{array}{l}0.270921 \\
0.272633 \\
0.274356 \\
0.276093 \\
0.277841\end{array}$ & $\begin{array}{l}3360 \\
3370 \\
3380 \\
3390 \\
3400\end{array}$ & $\begin{array}{l}120.9 \\
121.1 \\
121.3 \\
121.5 \\
121.7\end{array}$ & $\begin{array}{l}226 \\
802 \\
377 \\
955 \\
530\end{array}$ & $\begin{array}{l}3360 \\
3370 \\
3380 \\
3390 \\
3400\end{array}$ & $\begin{array}{l}165.6 \\
166.1 \\
166.6 \\
167.1 \\
167.6\end{array}$ \\
\hline $\begin{array}{l}0.688916 \\
0.691513 \\
0.694114 \\
0.696721 \\
0.699330\end{array}$ & $\begin{array}{l}3410 \\
3420 \\
3430 \\
3440 \\
3450\end{array}$ & $\begin{array}{l}7.6 \\
7.6 \\
7.6 \\
7.6 \\
7.6\end{array}$ & $\begin{array}{l}26.52416 \\
26.79303 \\
27.06455 \\
27.33873 \\
27.61555\end{array}$ & $\begin{array}{l}3410 \\
3420 \\
3430 \\
3440\end{array}$ & $\begin{array}{l}76.5 \\
76.7 \\
77.0 \\
77.2 \\
77.4\end{array}$ & $\begin{array}{l}0.279602 \\
0.281376 \\
0.283162 \\
0.284961 \\
0.286772\end{array}$ & $\begin{array}{l}3410 \\
3420 \\
3430 \\
3440 \\
3450\end{array}$ & $\begin{array}{l}121.9 \\
122.2 \\
122.4 \\
122.6 \\
122.8\end{array}$ & $\begin{array}{l}0.181106 \\
0.181683 \\
0.182260 \\
0.182839 \\
0.183415\end{array}$ & $\begin{array}{l}3410 \\
3420 \\
3430 \\
3440 \\
3450\end{array}$ & $\begin{array}{l}168.1 \\
168.6 \\
169.1 \\
169.6 \\
170.1\end{array}$ \\
\hline $\begin{array}{l}0.701944 \\
0.704561 \\
0.707183 \\
0.709808 \\
0.712439\end{array}$ & $\begin{array}{l}3460 \\
3470 \\
3480 \\
3490 \\
3500\end{array}$ & $\begin{array}{l}7.7 \\
7.7 \\
7.7 \\
7.7 \\
7.8\end{array}$ & $\begin{array}{l}27.89511 \\
28.17737 \\
28.46241 \\
28.75024 \\
29.04086\end{array}$ & $\begin{array}{l}3460 \\
3470 \\
3480 \\
3490 \\
3500\end{array}$ & $\begin{array}{l}77.6 \\
77.9 \\
78.1 \\
78.3 \\
78.5\end{array}$ & $\begin{array}{l}0.288596 \\
0.290434 \\
0.292284 \\
0.294148 \\
0.296024\end{array}$ & $\begin{array}{l}3460 \\
3470 \\
3480 \\
3490 \\
3500\end{array}$ & $\begin{array}{l}123.1 \\
123.3 \\
123.5 \\
123.7 \\
123.9\end{array}$ & $\begin{array}{l}0.183992 \\
0.184571 \\
0.185148 \\
0.185727 \\
0.186307\end{array}$ & $\begin{array}{l}3460 \\
3470 \\
3480 \\
3490 \\
3500\end{array}$ & $\begin{array}{l}170.6 \\
171.1 \\
171.5 \\
172.0 \\
172.5\end{array}$ \\
\hline
\end{tabular}


$3510-4000$

Geologic age, in millions of years, calculated from the atomic ratios of indicated isotopes

\begin{tabular}{|c|c|c|c|c|c|c|c|c|c|c|c|}
\hline \multicolumn{3}{|c|}{$N_{206} / N_{238}$} & \multicolumn{3}{|c|}{$N_{207} / N_{235}$} & \multicolumn{3}{|c|}{$N_{207} / N_{206}$} & \multicolumn{3}{|c|}{$N_{208} / N_{232}$} \\
\hline \multirow[b]{2}{*}{ Ratio } & \multicolumn{2}{|c|}{ Age } & \multirow[b]{2}{*}{ Ratio } & \multicolumn{2}{|c|}{ Age } & \multirow[b]{2}{*}{ Ratio } & \multicolumn{2}{|c|}{ Age } & \multirow[b]{2}{*}{ Ratio } & \multicolumn{2}{|c|}{ Age } \\
\hline & \begin{tabular}{|c|}
$\begin{array}{c}\text { Number } \\
\text { of } \\
\text { years }\end{array}$ \\
\end{tabular} & $\begin{array}{c}\text { Error } \\
\pm\end{array}$ & & \begin{tabular}{|c|} 
Number \\
of \\
years
\end{tabular} & $\begin{array}{c}\text { Error } \\
\pm\end{array}$ & & \begin{tabular}{|c|}
$\begin{array}{c}\text { Number } \\
\text { of } \\
\text { years }\end{array}$ \\
\end{tabular} & $\begin{array}{c}\text { Error } \\
\pm\end{array}$ & & \begin{tabular}{|c|}
$\begin{array}{c}\text { Number } \\
\text { of } \\
\text { years }\end{array}$ \\
\end{tabular} & $\begin{array}{c}\text { Error } \\
\pm\end{array}$ \\
\hline $\begin{array}{l}0.715073 \\
0.717712 \\
0.720353 \\
0.722997 \\
0.725648\end{array}$ & $\begin{array}{l}3510 \\
3520 \\
3530 \\
3540 \\
3550\end{array}$ & $\begin{array}{l}7.8 \\
7.8 \\
7.8 \\
7.8 \\
7.9\end{array}$ & $\begin{array}{l}29.33434 \\
29.63065 \\
29.92990 \\
30.23203 \\
30.53715\end{array}$ & $\begin{array}{l}3510 \\
3520 \\
3530 \\
3540 \\
3550\end{array}$ & $\begin{array}{l}78.8 \\
79.0 \\
79.2 \\
79.4 \\
79.7\end{array}$ & $\begin{array}{l}0.297914 \\
0.299817 \\
0.301735 \\
0.303666 \\
0.305610\end{array}$ & $\begin{array}{l}3510 \\
3520 \\
3530 \\
3540 \\
3550\end{array}$ & $\begin{array}{l}124.2 \\
124.4 \\
124.6 \\
124.8 \\
125.0\end{array}$ & $\begin{array}{l}0.186885 \\
0.187466 \\
0.188045 \\
0.188625 \\
0.189206\end{array}$ & $\begin{array}{l}3510 \\
3520 \\
3530 \\
3540 \\
3550\end{array}$ & $\begin{array}{l}173.0 \\
173.5 \\
174.0 \\
174.5 \\
175.0\end{array}$ \\
\hline $\begin{array}{l}0.728304 \\
0.730961 \\
0.733623 \\
0.736292 \\
0.738961\end{array}$ & $\begin{array}{l}3560 \\
3570 \\
3580 \\
3590 \\
3600\end{array}$ & $\begin{array}{l}7.9 \\
7.9 \\
7.9 \\
8.0 \\
8.0\end{array}$ & $\begin{array}{l}30.84526 \\
31.15633 \\
31.47048 \\
31.78766 \\
32.10798\end{array}$ & $\begin{array}{l}3560 \\
3570 \\
3580 \\
3590 \\
3600\end{array}$ & $\begin{array}{l}79.9 \\
80.1 \\
80.3 \\
80.6 \\
80.8\end{array}$ & $\begin{array}{l}0.307568 \\
0.309540 \\
0.311527 \\
0.313526 \\
0.315542\end{array}$ & $\begin{array}{l}3560 \\
3570 \\
3580 \\
3590 \\
3600\end{array}$ & $\begin{array}{l}125.3 \\
125.5 \\
125.7 \\
125.9 \\
126.2\end{array}$ & $\begin{array}{l}0.189785 \\
0.190366 \\
0.190947 \\
0.191530 \\
0.192112\end{array}$ & $\begin{array}{l}3560 \\
3570 \\
3580 \\
3590 \\
3600\end{array}$ & $\begin{array}{l}175.5 \\
176.0 \\
176.5 \\
177.0 \\
177.5\end{array}$ \\
\hline $\begin{array}{l}0.741637 \\
0.744316 \\
0.746998 \\
0.749686 \\
0.752377\end{array}$ & $\begin{array}{l}3610 \\
3620 \\
3630 \\
3640 \\
3650\end{array}$ & $\begin{array}{l}8.0 \\
8.0 \\
8.0 \\
8.1 \\
8.1\end{array}$ & $\begin{array}{l}32.43143 \\
32.75800 \\
33.08779 \\
33.42078 \\
33.75705\end{array}$ & $\begin{array}{l}3610 \\
3620 \\
3630 \\
3640 \\
3650\end{array}$ & $\begin{array}{l}81.0 \\
81.2 \\
81.5 \\
81.7 \\
81.9\end{array}$ & $\begin{array}{l}0.317570 \\
0.319614 \\
0.321672 \\
0.323745 \\
0.325833\end{array}$ & $\begin{array}{l}3610 \\
3620 \\
3630 \\
3640 \\
3650\end{array}$ & $\begin{array}{l}126.4 \\
126.6 \\
126.8 \\
127.0 \\
127.3\end{array}$ & $\begin{array}{l}2694 \\
3276 \\
3859 \\
1441 \\
5023\end{array}$ & $\begin{array}{l}3610 \\
3620 \\
3630 \\
3640 \\
3650\end{array}$ & $\begin{array}{l}178.0 \\
178.5 \\
178.9 \\
179.4 \\
179.9\end{array}$ \\
\hline $\begin{array}{l}0.755070 \\
0.757770 \\
0.760474 \\
0.763182 \\
0.765894\end{array}$ & $\begin{array}{l}3660 \\
3670 \\
3680 \\
3690 \\
3700\end{array}$ & $\begin{array}{l}8.1 \\
8.1 \\
8.2 \\
8.2 \\
8.2\end{array}$ & $\begin{array}{l}34.09660 \\
34.43945 \\
34.78567 \\
35.13523 \\
35.48826\end{array}$ & $\begin{array}{l}3660 \\
3670 \\
3680 \\
3690 \\
3700\end{array}$ & $\begin{array}{l}82.1 \\
82.4 \\
82.6 \\
82.8 \\
83.0\end{array}$ & $\begin{array}{l}0.327936 \\
0.330053 \\
0.332186 \\
0.334334 \\
0.336497\end{array}$ & $\begin{array}{l}3660 \\
3670 \\
3680 \\
3690 \\
3700\end{array}$ & $\begin{array}{l}127.5 \\
127.7 \\
127.9 \\
128.2 \\
128.4\end{array}$ & $\begin{array}{l}0.1 \\
0.1 \\
0.1 \\
0.1 \\
0.1\end{array}$ & $\begin{array}{l}3660 \\
3670 \\
3680 \\
3690 \\
3700\end{array}$ & $\begin{array}{l}180.4 \\
180.9 \\
181.4 \\
181.9 \\
182.4\end{array}$ \\
\hline $\begin{array}{l}0.768610 \\
0.771329 \\
0.774056 \\
0.776784 \\
0.779517\end{array}$ & $\begin{array}{l}3710 \\
3720 \\
3730 \\
3740 \\
3750\end{array}$ & $\begin{array}{l}8.2 \\
8.2 \\
8.3 \\
8.3 \\
8.3\end{array}$ & $\begin{array}{l}473 \\
464 \\
811 \\
509 \\
569\end{array}$ & $\begin{array}{l}3710 \\
3720 \\
3730 \\
3740 \\
3750\end{array}$ & $\begin{array}{l}83.3 \\
83.5 \\
83.7 \\
83.9\end{array}$ & $\begin{array}{l}8676 \\
0871 \\
3080 \\
5306 \\
7548\end{array}$ & $\begin{array}{l}3710 \\
3720 \\
3730 \\
3740 \\
3750\end{array}$ & $\begin{array}{l}128.6 \\
128.8 \\
129.0 \\
129.3 \\
129.5\end{array}$ & $\begin{array}{l}0.1 \\
0.1 \\
0.1 \\
0.2 \\
0.2\end{array}$ & $\begin{array}{l}3710 \\
3720 \\
3730 \\
3740 \\
3750\end{array}$ & $\begin{array}{l}182.9 \\
183.4 \\
183.9 \\
184.4 \\
184.9\end{array}$ \\
\hline $\begin{array}{l}0.782254 \\
0.784996 \\
0.787740 \\
0.790489 \\
0.793244\end{array}$ & $\begin{array}{l}3760 \\
3770 \\
3780 \\
3790 \\
3800\end{array}$ & $\begin{array}{l}8.3 \\
8.4 \\
8.4 \\
8.4 \\
8.4\end{array}$ & $\begin{array}{l}37.67992 \\
38.05776 \\
38.43933 \\
38.82459 \\
39.21365\end{array}$ & $\begin{array}{l}3760 \\
3770 \\
3780 \\
3790 \\
3800\end{array}$ & $\begin{array}{l}84.4 \\
84.6 \\
84.8 \\
850\end{array}$ & $\begin{array}{l}0.349806 \\
0.352080 \\
0.354371 \\
0.356678 \\
0.359001\end{array}$ & $\begin{array}{l}3760 \\
3770 \\
3780 \\
3790 \\
3800\end{array}$ & $\begin{array}{l}129.7 \\
129.9 \\
130.2 \\
130.4 \\
130.6\end{array}$ & $\begin{array}{l}0.2 \\
0.2 \\
0.2 \\
0.2 \\
0.2\end{array}$ & $\begin{array}{l}3760 \\
3770 \\
3780 \\
3790 \\
3800\end{array}$ & $\begin{array}{l}185.4 \\
185.8 \\
186.3 \\
186.8 \\
187.3\end{array}$ \\
\hline $\begin{array}{l}0.796001 \\
0.798764 \\
0.801530 \\
0.804301 \\
0.807077\end{array}$ & $\begin{array}{l}3810 \\
3820 \\
3830 \\
3840 \\
3850\end{array}$ & $\begin{array}{l}8.4 \\
8.5 \\
8.5 \\
8.5 \\
8.5\end{array}$ & $\begin{array}{l}39.60652 \\
40.00318 \\
40.40375 \\
40.80820 \\
41.21664\end{array}$ & $\begin{array}{l}3810 \\
3820 \\
3830 \\
3840 \\
3850\end{array}$ & $\begin{array}{l}85.5 \\
85.7 \\
85.9 \\
86.2 \\
86.4\end{array}$ & $\begin{array}{l}0.361342 \\
0.363698 \\
0.366073 \\
0.368463 \\
0.370871\end{array}$ & $\begin{array}{l}3810 \\
3820 \\
3830 \\
3840 \\
3850\end{array}$ & $\begin{array}{l}130.8 \\
131.1 \\
131.3 \\
131.5 \\
131.7\end{array}$ & $\begin{array}{l}0.204393 \\
0.204982 \\
0.205571 \\
0.206159 \\
0.206748\end{array}$ & $\begin{array}{l}3810 \\
3820 \\
3830 \\
3840 \\
3850\end{array}$ & $\begin{array}{l}187.8 \\
188.3 \\
188.8 \\
189.3 \\
189.8\end{array}$ \\
\hline $\begin{array}{l}0.809857 \\
0.812641 \\
0.815430 \\
0.818222 \\
0.821018\end{array}$ & $\begin{array}{l}3860 \\
3870 \\
3880 \\
3890 \\
3900\end{array}$ & $\begin{array}{l}8.6 \\
8.6 \\
8.6 \\
8.6 \\
8.6\end{array}$ & $\begin{array}{l}41.62907 \\
42.04549 \\
42.46602 \\
42.89062 \\
43.31940\end{array}$ & $\begin{array}{l}3860 \\
3870 \\
3880 \\
3890 \\
3900\end{array}$ & $\begin{array}{l}86.6 \\
86.8 \\
87.1 \\
87.3 \\
87.5\end{array}$ & $\begin{array}{l}0.373296 \\
0.375739 \\
0.378199 \\
0.380677 \\
0.383173\end{array}$ & $\begin{array}{l}3860 \\
3870 \\
3880 \\
3890 \\
3900\end{array}$ & $\begin{array}{l}132.0 \\
132.2 \\
132.4 \\
132.6 \\
132.9\end{array}$ & $\begin{array}{l}0.207336 \\
0.207927 \\
0.208517 \\
0.209108 \\
0.209697\end{array}$ & $\begin{array}{l}3860 \\
3870 \\
3880 \\
3890 \\
3900\end{array}$ & $\begin{array}{l}190.3 \\
190.8 \\
191.3 \\
191.8 \\
192.3\end{array}$ \\
\hline $\begin{array}{l}0.823817 \\
0.826623 \\
0.829432 \\
0.832247 \\
0.835065\end{array}$ & $\begin{array}{l}3910 \\
3920 \\
3930 \\
3940 \\
3950\end{array}$ & $\begin{array}{l}8.7 \\
8.7 \\
8.7 \\
8.7 \\
8.8\end{array}$ & $\begin{array}{l}43.75238 \\
44.18953 \\
44.63101 \\
45.07675 \\
45.52689\end{array}$ & $\begin{array}{l}3910 \\
3920 \\
3930 \\
3940 \\
3950\end{array}$ & $\begin{array}{l}87.7 \\
88.0 \\
88.2 \\
88.4 \\
88.6\end{array}$ & $\begin{array}{l}0.385688 \\
0.388220 \\
0.390770 \\
0.393338 \\
0.395925\end{array}$ & $\begin{array}{l}3910 \\
3920 \\
3930 \\
3940 \\
3950\end{array}$ & $\begin{array}{l}133.1 \\
133.3 \\
133.5 \\
133.8 \\
134.0\end{array}$ & $\begin{array}{l}0.210288 \\
0.210879 \\
0.211470 \\
0.212061 \\
0.212653\end{array}$ & $\begin{array}{l}3910 \\
3920 \\
3930 \\
3940 \\
3950\end{array}$ & $\begin{array}{l}192.7 \\
193.2 \\
193.7 \\
194.2 \\
194.7\end{array}$ \\
\hline $\begin{array}{l}837887 \\
840715 \\
843546 \\
846382 \\
849221\end{array}$ & $\begin{array}{l}3960 \\
3970 \\
3980 \\
3990 \\
4000\end{array}$ & $\begin{array}{l}8.8 \\
8.8 \\
8.8 \\
8.8 \\
8.9\end{array}$ & $\begin{array}{l}45.98144 \\
46.44037 \\
46.90383 \\
47.37178 \\
47.84434\end{array}$ & $\begin{array}{l}3960 \\
3970 \\
3980 \\
3990 \\
4000\end{array}$ & $\begin{array}{l}88.9 \\
89.1 \\
89.3 \\
89.5 \\
89.8\end{array}$ & $\begin{array}{l}0.398532 \\
0.401155 \\
0.403799 \\
0.406461 \\
0.409143\end{array}$ & $\begin{array}{l}3960 \\
3970 \\
3980 \\
3990 \\
4000\end{array}$ & $\begin{array}{l}134.2 \\
134.4 \\
134.7 \\
134.9 \\
135.1\end{array}$ & $\begin{array}{l}0.213245 \\
0.213838 \\
0.214431 \\
0.215023 \\
0.215616\end{array}$ & $\begin{array}{l}3960 \\
3970 \\
3980 \\
3990 \\
4000\end{array}$ & $\begin{array}{l}195.2 \\
195.7 \\
196.2 \\
196.7 \\
197.2\end{array}$ \\
\hline
\end{tabular}


Geologic age, in millions of years, calculated from the atomic ratios of indicated isotopes

\begin{tabular}{|c|c|c|c|c|c|c|c|c|c|c|c|}
\hline \multicolumn{3}{|c|}{$N_{206} / N_{238}$} & \multicolumn{3}{|c|}{$N_{207} / N_{235}$} & \multicolumn{3}{|c|}{$N_{207} / N_{206}$} & \multicolumn{3}{|c|}{$N_{208} / N_{232}$} \\
\hline \multirow[b]{2}{*}{ Ratio } & \multicolumn{2}{|c|}{ Age } & \multirow[b]{2}{*}{ Ratio } & \multicolumn{2}{|c|}{ Age } & \multirow[b]{2}{*}{ Ratio } & \multicolumn{2}{|c|}{ Age } & \multirow[b]{2}{*}{ Ratio } & \multicolumn{2}{|c|}{ Age } \\
\hline & \begin{tabular}{|c|}
$\begin{array}{c}\text { Number } \\
\text { of } \\
\text { years }\end{array}$ \\
\end{tabular} & $\begin{array}{c}\text { Error } \\
\pm\end{array}$ & & \begin{tabular}{|c|} 
Number \\
of \\
years
\end{tabular} & $\begin{array}{c}\text { Error } \\
\pm\end{array}$ & & $\begin{array}{c}\text { Number } \\
\text { of } \\
\text { years }\end{array}$ & $\begin{array}{c}\text { Error } \\
\pm\end{array}$ & & \begin{tabular}{|c|}
$\begin{array}{c}\text { Number } \\
\text { of } \\
\text { years }\end{array}$ \\
\end{tabular} & $\begin{array}{c}\text { Error } \\
\pm\end{array}$ \\
\hline $\begin{array}{l}0.852067 \\
0.854915 \\
0.857765 \\
0.860626 \\
0.863486\end{array}$ & $\begin{array}{l}4010 \\
4020 \\
4030 \\
4040 \\
4050\end{array}$ & $\begin{array}{l}8.9 \\
8.9 \\
8.9 \\
9.0 \\
9.0\end{array}$ & $\begin{array}{l}48.32153 \\
48.80331 \\
49.28987 \\
49.78112 \\
50.27722\end{array}$ & $\begin{array}{l}4010 \\
4020 \\
4030 \\
4040 \\
4050\end{array}$ & $\begin{array}{l}90.0 \\
90.2 \\
90.4 \\
90.7 \\
90.9\end{array}$ & $\begin{array}{l}44 \\
64 \\
06 \\
64 \\
45\end{array}$ & $\begin{array}{l}4010 \\
4020 \\
4030 \\
4040 \\
4050\end{array}$ & $\begin{array}{l}13 \\
13 \\
13 \\
13 \\
13\end{array}$ & $\begin{array}{l}210 \\
803 \\
397 \\
994 \\
587\end{array}$ & $\begin{array}{l}4010 \\
4020 \\
4030 \\
4040 \\
4050\end{array}$ & $\begin{array}{l}197.7 \\
198.2 \\
198.7 \\
199.2 \\
199.6\end{array}$ \\
\hline $\begin{array}{l}0.866352 \\
0.869224 \\
0.872099 \\
0.874978 \\
0.877861\end{array}$ & $\begin{array}{l}4060 \\
4070 \\
4080 \\
4090 \\
4100\end{array}$ & $\begin{array}{l}9.0 \\
9.0 \\
9.0 \\
9.1 \\
9.1\end{array}$ & $\begin{array}{l}7817 \\
3396 \\
474 \\
046 \\
3127\end{array}$ & $\begin{array}{l}4060 \\
4070 \\
4080 \\
4090 \\
4100\end{array}$ & $\begin{array}{l}91.1 \\
91.3 \\
91.6 \\
91.8 \\
92.0\end{array}$ & $\begin{array}{l}546 \\
465 \\
306 \\
167 \\
50\end{array}$ & $\begin{array}{l}4060 \\
4070 \\
4080 \\
4090 \\
4100\end{array}$ & $\begin{array}{l}13 \\
13 \\
13 \\
13 \\
13\end{array}$ & $\begin{array}{l}183 \\
776 \\
372 \\
969 \\
563\end{array}$ & $\begin{array}{l}4060 \\
4070 \\
4080 \\
4090 \\
4100\end{array}$ & $\begin{array}{l}200.1 \\
200.6 \\
201.1 \\
201.6 \\
202.1\end{array}$ \\
\hline $\begin{array}{l}0.880750 \\
0.883644 \\
0.886540 \\
0.889443 \\
0.892347\end{array}$ & $\begin{array}{l}4110 \\
4120 \\
4130 \\
4140 \\
4150\end{array}$ & $\begin{array}{l}9.1 \\
9.1 \\
9.2 \\
9.2 \\
9.2\end{array}$ & $\begin{array}{l}53.35717 \\
53.88816 \\
54.42438 \\
54.96579 \\
55.51254\end{array}$ & $\begin{array}{l}4110 \\
4120 \\
4130 \\
4140 \\
4150\end{array}$ & $\begin{array}{l}92.2 \\
92.5 \\
92.7 \\
92.9 \\
93.1\end{array}$ & $\begin{array}{l}52 \\
75 \\
21 \\
87 \\
76\end{array}$ & $\begin{array}{l}4110 \\
4120 \\
4130 \\
4140 \\
4150\end{array}$ & $\begin{array}{l}13 \\
13 \\
13 \\
13 \\
13\end{array}$ & $\begin{array}{l}60 \\
58 \\
55 \\
52 \\
50\end{array}$ & $\begin{array}{l}4110 \\
4120 \\
4130 \\
4140 \\
4150\end{array}$ & $\begin{array}{l}202.6 \\
203.1 \\
203.6 \\
204.1 \\
204.6\end{array}$ \\
\hline $\begin{array}{l}0.895257 \\
0.898172 \\
0.901092 \\
0.904017 \\
0.906946\end{array}$ & $\begin{array}{l}4160 \\
4170 \\
4180 \\
4190 \\
4200\end{array}$ & $\begin{array}{l}9.2 \\
9.2 \\
9.3 \\
9.3 \\
9.3\end{array}$ & $\begin{array}{l}64 \\
07 \\
01 \\
38 \\
36\end{array}$ & $\begin{array}{l}4160 \\
4170 \\
4180 \\
4190 \\
4200\end{array}$ & $\begin{array}{l}93 \\
93 \\
93 \\
94\end{array}$ & $\begin{array}{l}86 \\
17 \\
70 \\
45 \\
43\end{array}$ & $\begin{array}{l}160 \\
170 \\
180 \\
190 \\
200\end{array}$ & $\begin{array}{l}13 \\
13 \\
13 \\
13 \\
13\end{array}$ & $\begin{array}{l}47 \\
45 \\
43 \\
42 \\
42\end{array}$ & $\begin{array}{l}4160 \\
4170 \\
4180 \\
4190 \\
4200\end{array}$ & $\begin{array}{l}205.1 \\
205.6 \\
206.1 \\
206.5 \\
207.0\end{array}$ \\
\hline $\begin{array}{l}0.909879 \\
0.912817 \\
0.915759 \\
0.918705 \\
0.921655\end{array}$ & $\begin{array}{l}4210 \\
4220 \\
4230 \\
4240 \\
4250\end{array}$ & $\begin{array}{l}9.3 \\
9.4 \\
9.4 \\
9.4 \\
9.4\end{array}$ & $\begin{array}{l}96 \\
15 \\
12 \\
80 \\
38\end{array}$ & $\begin{array}{l}4210 \\
4220 \\
4230 \\
4240 \\
4250\end{array}$ & 94 & $\begin{array}{l}63 \\
06 \\
72 \\
60 \\
73\end{array}$ & $\begin{array}{l}4210 \\
4220 \\
4230 \\
4240 \\
4250\end{array}$ & $\begin{array}{l}13 \\
14 \\
14 \\
14 \\
14\end{array}$ & & $\begin{array}{l}4210 \\
4220 \\
4230 \\
4240 \\
4250\end{array}$ & $\begin{array}{l}20 \\
20 \\
20 \\
20 \\
20\end{array}$ \\
\hline $\begin{array}{l}0.924613 \\
0.927571 \\
0.930537 \\
0.933506 \\
0.936480\end{array}$ & $\begin{array}{l}4260 \\
4270 \\
4280 \\
4290 \\
4300\end{array}$ & $\begin{array}{l}9.4 \\
9.5 \\
9.5 \\
9.5 \\
9.5\end{array}$ & $\begin{array}{l}85 \\
19 \\
60 \\
01 \\
59\end{array}$ & $\begin{array}{l}4260 \\
4270 \\
4280 \\
4290 \\
4300\end{array}$ & $\begin{array}{l}9 \\
9 \\
9\end{array}$ & $\begin{array}{l}07 \\
367 \\
49 \\
55 \\
86\end{array}$ & $\begin{array}{l}4260 \\
4270 \\
4280 \\
4290 \\
4300\end{array}$ & $\begin{array}{l}14 \\
14 \\
14 \\
14 \\
14\end{array}$ & & $\begin{array}{l}4260 \\
4270 \\
4280 \\
4290 \\
4300\end{array}$ & $\begin{array}{l}210 . \\
210 \\
211 . \\
211 \\
212 .\end{array}$ \\
\hline $\begin{array}{l}0.939459 \\
0.942441 \\
0.945430 \\
0.948422 \\
0.951420\end{array}$ & $\begin{array}{l}4310 \\
4320 \\
4330 \\
4340 \\
4350\end{array}$ & $\begin{array}{l}9.6 \\
9.6 \\
9.6 \\
9.6 \\
9.6\end{array}$ & $\begin{array}{l}65.0 \\
65.6 \\
66.3 \\
66.9 \\
67.6\end{array}$ & $\begin{array}{l}4310 \\
4320 \\
4330 \\
4340 \\
4350\end{array}$ & $\begin{array}{l}96 \\
96 \\
97 \\
97 \\
97\end{array}$ & $\begin{array}{l}40 \\
20 \\
23 \\
51 \\
04\end{array}$ & $\begin{array}{l}0 \\
0 \\
0 \\
0 \\
0\end{array}$ & $\begin{array}{l}14 \\
14 \\
14 \\
14 \\
14\end{array}$ & & $\begin{array}{l}4310 \\
4320 \\
4330 \\
4340 \\
4350\end{array}$ & $\begin{array}{l}212 . \\
213 . \\
213 . \\
213 . \\
214 .\end{array}$ \\
\hline $\begin{array}{l}0.954419 \\
0.957428 \\
0.960438 \\
0.963453 \\
0.966472\end{array}$ & $\begin{array}{l}4360 \\
4370 \\
4380 \\
4390 \\
4400\end{array}$ & $\begin{array}{l}9.7 \\
9.7 \\
9.7 \\
9.7 \\
9.8\end{array}$ & $\begin{array}{l}68.3 \\
68.9 \\
69.6 \\
70.3 \\
71.0\end{array}$ & $\begin{array}{l}4360 \\
4370 \\
4380\end{array}$ & $\begin{array}{l}97.8 \\
98.1 \\
98.3 \\
98.5 \\
98.7\end{array}$ & $\begin{array}{l}784 \\
286 \\
816 \\
371 \\
953\end{array}$ & $\begin{array}{l}4360 \\
4370 \\
4380 \\
4390 \\
4400\end{array}$ & $\begin{array}{l}14 \\
14 \\
14 \\
14 \\
14\end{array}$ & $\begin{array}{l}7167 \\
7771 \\
3375 \\
3980 \\
9584\end{array}$ & $\begin{array}{l}4360 \\
4370 \\
4380 \\
4390 \\
4400\end{array}$ & $\begin{array}{l}214.9 \\
215.4 \\
215.9 \\
216.4 \\
216.9\end{array}$ \\
\hline $\begin{array}{l}0.969496 \\
0.972527 \\
0.975562 \\
0.978598\end{array}$ & $\begin{array}{l}4410 \\
4420 \\
4430 \\
4440 \\
4450\end{array}$ & $\begin{array}{l}9.8 \\
9.8 \\
9.8 \\
9.8 \\
9.9\end{array}$ & $\begin{array}{l}71.76425 \\
72.47503 \\
73.19284 \\
73.91758 \\
74.64949\end{array}$ & $\begin{array}{l}4410 \\
4420 \\
4430 \\
4440 \\
4450\end{array}$ & $\begin{array}{l}99.0 \\
99.2 \\
99.4 \\
99.6 \\
99.9\end{array}$ & $\begin{array}{l}61 \\
93 \\
53 \\
41 \\
55\end{array}$ & $\begin{array}{l}4410 \\
4420 \\
4430 \\
4440 \\
4450\end{array}$ & $\begin{array}{l}14 \\
14 \\
14 \\
14 \\
14\end{array}$ & $\begin{array}{l}90 \\
96 \\
02 \\
07 \\
14\end{array}$ & $\begin{array}{l}4410 \\
4420 \\
4430 \\
4440 \\
4450\end{array}$ & $\begin{array}{l}217.4 \\
217.9 \\
218.4 \\
218.9 \\
219.4\end{array}$ \\
\hline $\begin{array}{l}0.984690 \\
0.987743 \\
0.990800 \\
0.993862 \\
0.996930\end{array}$ & $\begin{array}{l}4460 \\
4470 \\
4480 \\
4490 \\
4500\end{array}$ & $\begin{array}{r}9.9 \\
999 \\
9.9 \\
10.0 \\
10.0\end{array}$ & $\begin{array}{l}75.38854 \\
76.13473 \\
76.88829 \\
77.64914 \\
78.41750\end{array}$ & $\begin{array}{l}4460 \\
4470 \\
4480 \\
4490 \\
4500\end{array}$ & $\begin{array}{l}100.1 \\
100.3 \\
100.5 \\
100.8 \\
101.0\end{array}$ & $\begin{array}{l}0.555996 \\
0.559763 \\
0.563560 \\
0.567383 \\
0.571234\end{array}$ & $\begin{array}{l}4460 \\
4470 \\
4480 \\
4490 \\
4500\end{array}$ & $\begin{array}{l}145.6 \\
145.8 \\
146.0 \\
146.3 \\
146.5\end{array}$ & $\begin{array}{l}0.243221 \\
0.243828 \\
0.244435 \\
0.245044 \\
0.245651\end{array}$ & $\begin{array}{l}4460 \\
4470 \\
4480 \\
4490 \\
4500\end{array}$ & $\begin{array}{l}219.9 \\
220.4 \\
220.8 \\
221.3 \\
221.8\end{array}$ \\
\hline
\end{tabular}


$4515-5250$

Geologic age, in millions of years, calculated from the atomic ratios of indicated isotopes

\begin{tabular}{|c|c|c|c|c|c|c|c|c|c|c|c|}
\hline \multicolumn{3}{|c|}{$N_{206} / N_{238}$} & \multicolumn{3}{|c|}{$N_{207} / N_{235}$} & \multicolumn{3}{|c|}{$N_{207} / N_{206}$} & \multicolumn{3}{|c|}{$N_{208} / N_{232}$} \\
\hline \multirow[b]{2}{*}{ Ratio } & \multicolumn{2}{|c|}{ Age } & \multirow[b]{2}{*}{ Ratio } & \multicolumn{2}{|c|}{ Age } & \multirow[b]{2}{*}{ Ratio } & \multicolumn{2}{|c|}{ Age } & \multirow[b]{2}{*}{ Ratio } & \multicolumn{2}{|c|}{ Age } \\
\hline & $\begin{array}{c}\text { Number } \\
\text { of } \\
\text { years }\end{array}$ & $\begin{array}{c}\text { Error } \\
\pm\end{array}$ & & \begin{tabular}{|c|}
$\begin{array}{c}\text { Number } \\
\text { of } \\
\text { years }\end{array}$ \\
\end{tabular} & $\begin{array}{c}\text { Error } \\
\pm\end{array}$ & & \begin{tabular}{|c|}
$\begin{array}{c}\text { Number } \\
\text { of } \\
\text { years }\end{array}$ \\
\end{tabular} & $\begin{array}{c}\text { Error } \\
\pm \\
\end{array}$ & & \begin{tabular}{|c|}
$\begin{array}{c}\text { Number } \\
\text { of } \\
\text { years }\end{array}$ \\
\end{tabular} & $\begin{array}{c}\text { Error } \\
\pm\end{array}$ \\
\hline $\begin{array}{l}1.001538 \\
1.006158 \\
1.010789 \\
1.015429 \\
1.020080\end{array}$ & $\begin{array}{l}4515 \\
4530 \\
4545 \\
4560 \\
4575\end{array}$ & $\begin{array}{l}10.0 \\
10.0 \\
10.1 \\
10.1 \\
10.1\end{array}$ & $\begin{array}{l}79.5840 \\
80.7678 \\
81.9688 \\
83.1876 \\
84.4243\end{array}$ & $\begin{array}{l}4515 \\
4530 \\
4545 \\
4560 \\
4575\end{array}$ & $\begin{array}{l}101.3 \\
101.7 \\
102.0 \\
102.3 \\
102.7\end{array}$ & $\begin{array}{l}7064 \\
2959 \\
8917 \\
4943 \\
1034\end{array}$ & $\begin{array}{l}4515 \\
4530 \\
4545 \\
4560 \\
4575\end{array}$ & $\begin{array}{l}146.9 \\
147.2 \\
147.5 \\
147.9 \\
148.2\end{array}$ & $\begin{array}{l}0.246564 \\
0.247475 \\
0.248389 \\
0.249304 \\
0.250220\end{array}$ & $\begin{array}{l}4515 \\
4530 \\
4545 \\
4560 \\
4575\end{array}$ & $\begin{array}{l}222.6 \\
223.3 \\
224.0 \\
224.8 \\
225.5\end{array}$ \\
\hline $\begin{array}{l}1.024745 \\
1.029416 \\
1.034100 \\
1.038795 \\
1.043499\end{array}$ & $\begin{array}{l}4590 \\
4605 \\
4620 \\
4635 \\
4650\end{array}$ & $\begin{array}{l}10.2 \\
10.2 \\
10.2 \\
10.3 \\
10.3\end{array}$ & $\begin{array}{l}85.6790 \\
86.9524 \\
88.2443 \\
89.5553 \\
90.8854\end{array}$ & $\begin{array}{l}4590 \\
4605 \\
4620 \\
4635 \\
4650\end{array}$ & $\begin{array}{l}103.0 \\
103.3 \\
103.7 \\
104.0 \\
104.3\end{array}$ & $\begin{array}{l}190 \\
418 \\
712 \\
076 \\
511\end{array}$ & $\begin{array}{l}4590 \\
4605 \\
4620 \\
4635 \\
4650\end{array}$ & $\begin{array}{l}14 \\
14 \\
14 \\
14 \\
15\end{array}$ & $\begin{array}{l}134 \\
051 \\
967 \\
887 \\
805\end{array}$ & $\begin{array}{l}4590 \\
4605 \\
4620 \\
4635 \\
4650\end{array}$ & $\begin{array}{l}227.0 \\
227.7 \\
228.5 \\
229.2\end{array}$ \\
\hline $\begin{array}{l}1.048218 \\
1.052944 \\
1.057683 \\
1.062431 \\
1.067192\end{array}$ & $\begin{array}{l}4665 \\
4680 \\
4695 \\
4710 \\
4725\end{array}$ & $\begin{array}{l}10.3 \\
10.4 \\
10.4 \\
10.4 \\
10.5\end{array}$ & $\begin{array}{l}351 \\
047 \\
047 \\
943 \\
045 \\
352\end{array}$ & $\begin{array}{l}4665 \\
4680 \\
4695 \\
4710 \\
4725\end{array}$ & $\begin{array}{l}104.7 \\
105.0 \\
105.4 \\
105.7 \\
106.0\end{array}$ & $\begin{array}{l}014 \\
592 \\
241 \\
965 \\
762\end{array}$ & $\begin{array}{l}4665 \\
4680 \\
4695 \\
4710 \\
4725\end{array}$ & $\begin{array}{l}150.3 \\
150.7 \\
151.0 \\
151.3 \\
151.7\end{array}$ & $\begin{array}{l}0.255723 \\
0.256643 \\
0.257564 \\
0.258486 \\
0.259407\end{array}$ & $\begin{array}{l}4665 \\
4680 \\
4695 \\
4710 \\
4725\end{array}$ & $\begin{array}{l}230.7 \\
231.4 \\
232.2 \\
232.9\end{array}$ \\
\hline $\begin{array}{l}1.071963 \\
1.076746 \\
1.081538 \\
1.086344 \\
1.091157\end{array}$ & $\begin{array}{l}4740 \\
4755 \\
4770 \\
4785 \\
4800\end{array}$ & $\begin{array}{l}10.5 \\
10.5 \\
10.6 \\
10.6 \\
10.6\end{array}$ & $\begin{array}{l}870 \\
602 \\
550 \\
718 \\
107\end{array}$ & $\begin{array}{l}4740 \\
4755 \\
4770 \\
4785 \\
4800\end{array}$ & $\begin{array}{l}106.4 \\
106.7 \\
107.0 \\
107.4 \\
107.7\end{array}$ & $\begin{array}{l}634 \\
582 \\
608 \\
710 \\
892\end{array}$ & $\begin{array}{l}4740 \\
4755 \\
4770 \\
4785 \\
4800\end{array}$ & $\begin{array}{l}152.0 \\
152.4 \\
152.7 \\
153.1 \\
153.4\end{array}$ & $\begin{array}{l}330 \\
252 \\
175 \\
100 \\
026\end{array}$ & $\begin{array}{l}4740 \\
4755 \\
4770 \\
4785 \\
4800\end{array}$ & $\begin{array}{l}233.7 \\
234.4 \\
235.1 \\
235.9 \\
236.6\end{array}$ \\
\hline $\begin{array}{l}1.095986 \\
1.100823 \\
1.105671 \\
1.110532 \\
1.115402\end{array}$ & $\begin{array}{l}4815 \\
4830 \\
4845 \\
4860 \\
4875\end{array}$ & $\begin{array}{l}10.7 \\
10.7 \\
10.7 \\
10.8 \\
10.8\end{array}$ & $\begin{array}{l}723 \\
569 \\
647 \\
963 \\
516\end{array}$ & $\begin{array}{l}4815 \\
4830 \\
4845 \\
4860 \\
4875\end{array}$ & $\begin{array}{l}108.1 \\
108.4 \\
108.7 \\
109.1 \\
109.4\end{array}$ & $\begin{array}{l}152 \\
494 \\
917 \\
422 \\
010\end{array}$ & $\begin{array}{l}4815 \\
4830 \\
4845 \\
4860 \\
4875\end{array}$ & $\begin{array}{l}153.8 \\
154.1 \\
154.5 \\
154.8 \\
155.2\end{array}$ & $\begin{array}{l}951 \\
879 \\
806 \\
734 \\
662\end{array}$ & $\begin{array}{l}4815 \\
4830 \\
4845 \\
4860 \\
4875\end{array}$ & $\begin{array}{l}237.4 \\
238.1 \\
238.8 \\
239.6 \\
240.3\end{array}$ \\
\hline $\begin{array}{l}120283 \\
125177 \\
130084 \\
135001 \\
139928\end{array}$ & $\begin{array}{l}4890 \\
4905 \\
4920 \\
4935 \\
4950\end{array}$ & $\begin{array}{l}10.8 \\
10.9 \\
10.9 \\
10.9 \\
11.0\end{array}$ & $\begin{array}{l}115.0314 \\
116.7358 \\
118.4652 \\
120.2202 \\
122.0007\end{array}$ & $\begin{array}{l}4890 \\
4905 \\
4920 \\
4935 \\
4950\end{array}$ & $\begin{array}{l}109.7 \\
110.1 \\
110.4 \\
110.7 \\
111.1\end{array}$ & $\begin{array}{l}683 \\
441 \\
283 \\
214 \\
233\end{array}$ & $\begin{array}{l}4890 \\
4905 \\
4920 \\
4935 \\
4950\end{array}$ & $\begin{array}{l}15 \\
15 \\
15 \\
15 \\
15\end{array}$ & $\begin{array}{l}592 \\
521 \\
451 \\
383 \\
316\end{array}$ & $\begin{array}{l}4890 \\
4905 \\
4920 \\
4935 \\
4950\end{array}$ & $\begin{array}{l}241.8 \\
242.5 \\
243.3 \\
244.0\end{array}$ \\
\hline $\begin{array}{l}144866 \\
149817 \\
154777 \\
159751 \\
164737\end{array}$ & $\begin{array}{l}4965 \\
4980 \\
4995 \\
5010 \\
5025\end{array}$ & $\begin{array}{l}11.0 \\
11.0 \\
11.1 \\
11.1 \\
11.1\end{array}$ & $\begin{array}{l}123 . \\
125 . \\
127 . \\
129 . \\
131 .\end{array}$ & $\begin{array}{l}4965 \\
4980 \\
4995 \\
5010 \\
5025\end{array}$ & $\begin{array}{l}111.4 \\
111.8 \\
112.1 \\
112.4 \\
112.8\end{array}$ & $\begin{array}{l}0.785341 \\
0.793539 \\
0.801829 \\
0.810210 \\
0.818684\end{array}$ & $\begin{array}{l}4965 \\
4980 \\
4995 \\
5010 \\
5025\end{array}$ & $\begin{array}{l}15 \\
15 \\
15 \\
15 \\
15\end{array}$ & $\begin{array}{l}247 \\
182 \\
115 \\
050 \\
985\end{array}$ & $\begin{array}{l}4965 \\
4980 \\
4995 \\
5010 \\
5025\end{array}$ & $\begin{array}{l}244.8 \\
245.5 \\
246.2 \\
247.0 \\
247.7\end{array}$ \\
\hline $\begin{array}{l}169733 \\
174743 \\
179759 \\
184791 \\
189836\end{array}$ & $\begin{array}{l}5040 \\
5055 \\
5070 \\
5085 \\
5100\end{array}$ & $\begin{array}{l}11.2 \\
11.2 \\
11.2 \\
11.3 \\
11.3\end{array}$ & $\begin{array}{l}133.2474 \\
135.2195 \\
137.2204 \\
139.2508 \\
141_{13} 1_{10}\end{array}$ & $\begin{array}{l}5040 \\
5055 \\
5070 \\
5085 \\
5100\end{array}$ & $\begin{array}{l}113.1 \\
113.4 \\
113.8 \\
114.1 \\
114.4\end{array}$ & $\begin{array}{l}0.827252 \\
0.835915 \\
0.844678 \\
0.853536 \\
0.862491\end{array}$ & $\begin{array}{l}5040 \\
5055 \\
5070 \\
5085 \\
5100\end{array}$ & $\begin{array}{l}159.0 \\
159.4 \\
159.7 \\
160.1 \\
160.4\end{array}$ & $\begin{array}{l}8921 \\
9858 \\
0795 \\
1734 \\
2673\end{array}$ & $\begin{array}{l}5040 \\
5055 \\
5070 \\
5085 \\
5100\end{array}$ & $\begin{array}{l}248.5 \\
249.2 \\
249.9 \\
250.7 \\
251.4\end{array}$ \\
\hline $\begin{array}{l}194887 \\
199953 \\
205033 \\
210122 \\
15222\end{array}$ & $\begin{array}{l}5115 \\
5130 \\
5145 \\
5160 \\
5175\end{array}$ & $\begin{array}{l}11.3 \\
11.4 \\
11.4 \\
11.4 \\
11.5\end{array}$ & $\begin{array}{l}143.40_{13} \\
145.5226 \\
147.6748 \\
149.8588 \\
152.0748\end{array}$ & $\begin{array}{l}5115 \\
5130 \\
5145 \\
5160 \\
5175\end{array}$ & $\begin{array}{l}114.8 \\
115.1 \\
115.5 \\
115.8 \\
116.1\end{array}$ & $\begin{array}{l}0.871550 \\
0.880708 \\
0.889966 \\
0.899330 \\
0.908798\end{array}$ & $\begin{array}{l}5115 \\
5130 \\
5145 \\
5160 \\
5175\end{array}$ & $\begin{array}{l}160.8 \\
161.1 \\
161.5 \\
161.8 \\
162.2\end{array}$ & $\begin{array}{l}0.283613 \\
0.284553 \\
0.285493 \\
0.286436 \\
0.287377\end{array}$ & $\begin{array}{l}5115 \\
5130 \\
5145 \\
5160 \\
5175\end{array}$ & $\begin{array}{l}252.1 \\
252.9 \\
253.6 \\
254.4\end{array}$ \\
\hline $\begin{array}{l}20333 \\
25459 \\
30598 \\
35744 \\
40905\end{array}$ & $\begin{array}{l}5190 \\
5205 \\
5220 \\
5235 \\
5250\end{array}$ & $\begin{array}{l}11.5 \\
11.5 \\
11.6 \\
11.6 \\
11.6\end{array}$ & $\begin{array}{l}154.3233 \\
156.6049 \\
158.9200 \\
161.2692 \\
163.6527\end{array}$ & $\begin{array}{l}5190 \\
5205 \\
5220 \\
5235 \\
5250\end{array}$ & $\begin{array}{l}116.5 \\
116.8 \\
117.1 \\
117.5 \\
117.8\end{array}$ & $\begin{array}{l}0.918373 \\
0.928053 \\
0.937839 \\
0.947739 \\
0.957747\end{array}$ & $\begin{array}{l}5190 \\
5205 \\
5220 \\
5235 \\
5250\end{array}$ & $\begin{array}{l}162.5 \\
162.9 \\
163.2 \\
163.6 \\
163.9\end{array}$ & $\begin{array}{l}0.288319 \\
0.289263 \\
0.290207 \\
0.291154 \\
0.292100\end{array}$ & $\begin{array}{l}5190 \\
5205 \\
5220 \\
5235 \\
5250\end{array}$ & $\begin{array}{l}255.8 \\
256.6 \\
257.3 \\
258.1 \\
258.8\end{array}$ \\
\hline
\end{tabular}


Geologic age, in millions of years, calculated from the atomic ratios of indicated isotopes

\begin{tabular}{|c|c|c|c|c|c|c|c|c|c|c|c|}
\hline \multicolumn{3}{|c|}{$N_{206} / N_{238}$} & \multicolumn{3}{|c|}{$N_{207} / N_{235}$} & \multicolumn{3}{|c|}{$N_{207} / N_{206}$} & \multicolumn{3}{|c|}{$N_{208} / N_{232}$} \\
\hline \multirow[b]{2}{*}{ Ratio } & \multicolumn{2}{|c|}{ Age } & \multirow[b]{2}{*}{ Ratio } & \multicolumn{2}{|c|}{ Age } & \multirow[b]{2}{*}{ Ratio } & \multicolumn{2}{|c|}{ Age } & \multirow[b]{2}{*}{ Ratio } & \multicolumn{2}{|c|}{ Age } \\
\hline & $\begin{array}{c}\text { Number } \\
\text { of } \\
\text { years }\end{array}$ & $\begin{array}{c}\text { Error } \\
\pm\end{array}$ & & \begin{tabular}{|c|} 
Number \\
of \\
years
\end{tabular} & $\begin{array}{c}\text { Error } \\
\pm\end{array}$ & & $\begin{array}{c}\text { Number } \\
\text { of } \\
\text { years }\end{array}$ & $\begin{array}{c}\text { Error } \\
\pm\end{array}$ & & \begin{tabular}{|c|}
$\begin{array}{c}\text { Number } \\
\text { of } \\
\text { years }\end{array}$ \\
\end{tabular} & $\begin{array}{c}\text { Error } \\
\pm\end{array}$ \\
\hline $\begin{array}{l}1.246076 \\
1.251259 \\
1.256457 \\
1.261664 \\
1.266883\end{array}$ & $\begin{array}{l}5265 \\
5280 \\
5295 \\
5310 \\
5325\end{array}$ & $\begin{array}{l}11.7 \\
11.7 \\
11.7 \\
11.8 \\
11.8\end{array}$ & $\begin{array}{l}166.0713 \\
168.5255 \\
171.0157 \\
173.5424 \\
176.1064\end{array}$ & $\begin{array}{l}5265 \\
5280 \\
5295 \\
5310 \\
5325\end{array}$ & $\begin{array}{l}118.1 \\
118.5 \\
118.8 \\
119.2 \\
119.5\end{array}$ & $\begin{array}{l}0.967868 \\
0.978103 \\
0.988449 \\
0.998913 \\
1.009496\end{array}$ & $\begin{array}{l}5265 \\
5280 \\
5295 \\
5310 \\
5325\end{array}$ & $\begin{array}{l}164.3 \\
164.6 \\
165.0 \\
165.3 \\
165.7\end{array}$ & $\begin{array}{l}0.293046 \\
0.293992 \\
0.294939 \\
0.295890 \\
0.296838\end{array}$ & $\begin{array}{l}5265 \\
5280 \\
5295 \\
5310 \\
5325\end{array}$ & $\begin{array}{l}259.5 \\
260.3 \\
261.0 \\
261.8 \\
262.5\end{array}$ \\
\hline $\begin{array}{l}1.272116 \\
1.277361 \\
1.282616 \\
1.287885 \\
1.293166\end{array}$ & $\begin{array}{l}5340 \\
5355 \\
5370 \\
5385 \\
5400\end{array}$ & $\begin{array}{l}11.8 \\
11.9 \\
11.9 \\
11.9 \\
12.0\end{array}$ & $\begin{array}{l}079 \\
477 \\
262 \\
440 \\
020\end{array}$ & $\begin{array}{l}5340 \\
5355 \\
5370 \\
5385 \\
5400\end{array}$ & $\begin{array}{l}119.8 \\
120.2 \\
120.5 \\
120.8 \\
121.2\end{array}$ & $\begin{array}{l}194 \\
014 \\
955 \\
018 \\
205\end{array}$ & $\begin{array}{l}5340 \\
5355 \\
5370 \\
5385 \\
5400\end{array}$ & $\begin{array}{l}166.0 \\
166.4 \\
166.7 \\
167.1 \\
167.4\end{array}$ & $\begin{array}{l}788 \\
738 \\
689 \\
642 \\
595\end{array}$ & $\begin{array}{l}5340 \\
5355 \\
5370 \\
5385 \\
5400\end{array}$ & $\begin{array}{l}263.2 \\
264.0 \\
264.7 \\
265.5 \\
266.2\end{array}$ \\
\hline $\begin{array}{l}1.298458 \\
1.303764 \\
1.309080 \\
1.314411 \\
1.319752\end{array}$ & $\begin{array}{l}5415 \\
5430 \\
5445 \\
5460 \\
5475\end{array}$ & $\begin{array}{l}12.0 \\
12.0 \\
12.1 \\
12.1 \\
12.1\end{array}$ & $\begin{array}{l}002 \\
398 \\
208 \\
442 \\
108\end{array}$ & $\begin{array}{l}5415 \\
5430 \\
5445 \\
5460 \\
5475\end{array}$ & $\begin{array}{l}121.5 \\
121.9 \\
122.2 \\
122.5 \\
122.9\end{array}$ & $\begin{array}{l}1.075518 \\
1.086958 \\
1.098527 \\
1.110223 \\
1122054\end{array}$ & $\begin{array}{l}5415 \\
5430 \\
5445 \\
5460 \\
5475\end{array}$ & $\begin{array}{l}16 \\
16 \\
16 \\
16 \\
16\end{array}$ & $\begin{array}{l}47 \\
401 \\
56 \\
13 \\
69\end{array}$ & $\begin{array}{l}5415 \\
5430 \\
5445 \\
5460 \\
5475\end{array}$ & $\begin{array}{l}266.9 \\
267.7 \\
268.4 \\
269.2 \\
269.9\end{array}$ \\
\hline $\begin{array}{l}1.325105 \\
1.330471 \\
1.335850 \\
1.341243 \\
1.346645\end{array}$ & $\begin{array}{l}5490 \\
5505 \\
5520 \\
5535 \\
5550\end{array}$ & $\begin{array}{l}12.2 \\
12.2 \\
12.2 \\
12.3 \\
12.3\end{array}$ & $\begin{array}{l}9207 \\
9750 \\
0740 \\
2185 \\
4094\end{array}$ & $\begin{array}{l}5490 \\
5505 \\
5520 \\
5535 \\
5550\end{array}$ & $\begin{array}{l}123.2 \\
123.5 \\
123.9 \\
124.2 \\
124.5\end{array}$ & $\begin{array}{l}017 \\
115 \\
1147 \\
315 \\
727 \\
227\end{array}$ & $\begin{array}{l}5490 \\
5505 \\
5520 \\
5535 \\
5550\end{array}$ & $\begin{array}{l}16 \\
16 \\
17 \\
17 \\
17\end{array}$ & $\begin{array}{l}24 \\
82 \\
40 \\
01 \\
60\end{array}$ & $\begin{array}{l}5490 \\
5505 \\
5520 \\
5535 \\
5550\end{array}$ & $\begin{array}{l}270.6 \\
271.4 \\
272.1 \\
272.9 \\
273.6\end{array}$ \\
\hline $\begin{array}{l}1.352062 \\
1.357491 \\
1.362931 \\
1.368385 \\
1.373851\end{array}$ & $\begin{array}{l}5565 \\
5580 \\
5595 \\
5610 \\
5625\end{array}$ & $\begin{array}{l}12.3 \\
12.4 \\
12.4 \\
12.4 \\
12.5\end{array}$ & $\begin{array}{l}469 \\
323 \\
657 \\
480 \\
302\end{array}$ & $\begin{array}{l}5565 \\
5580 \\
5595 \\
5610 \\
5625\end{array}$ & $\begin{array}{l}124.9 \\
125.2 \\
125.6 \\
125.9 \\
126.2\end{array}$ & $\begin{array}{l}1.1 \\
1.2 \\
1.2 \\
1.2 \\
1.2\end{array}$ & $\begin{array}{l}5565 \\
5580 \\
5595 \\
5610 \\
5625\end{array}$ & $\begin{array}{l}17 \\
17 \\
17 \\
17 \\
17\end{array}$ & $\left\{\begin{array}{l}19 \\
80 \\
42 \\
05 \\
68\end{array}\right.$ & $\begin{array}{l}5565 \\
5580 \\
5595 \\
5510 \\
5525\end{array}$ & $\begin{array}{l}274.3 \\
275.1 \\
275.8 \\
276.5 \\
277.3\end{array}$ \\
\hline $\begin{array}{r}1.379330 \\
1.384823 \\
1.390327 \\
1.395843 \\
1.401373\end{array}$ & $\begin{array}{l}5640 \\
5655 \\
5670 \\
5685 \\
5700\end{array}$ & $\begin{array}{l}12.5 \\
12.5 \\
12.6 \\
126 \\
12.6\end{array}$ & $\begin{array}{l}239.5627 \\
243.0965 \\
246.6820 \\
250.3202 \\
254.0120\end{array}$ & $\begin{array}{l}5640 \\
5655 \\
5670 \\
5685 \\
5700\end{array}$ & $\begin{array}{l}126.6 \\
126.9 \\
127.2 \\
127.6 \\
127.9\end{array}$ & $\begin{array}{l}96 \\
25 \\
06 \\
43 \\
335\end{array}$ & $\begin{array}{l}5640 \\
5655 \\
5670 \\
5685 \\
5700\end{array}$ & $\begin{array}{l}17 \\
17 \\
17 \\
17 \\
17\end{array}$ & & $\begin{array}{l}5540 \\
5555 \\
5570 \\
5685 \\
5700\end{array}$ & $\begin{array}{l}278.0 \\
278.8 \\
279.5 \\
280.2 \\
281.0\end{array}$ \\
\hline $\begin{array}{r}406915 \\
4412469 \\
418041 \\
423620 \\
429214\end{array}$ & $\begin{array}{l}5715 \\
5730 \\
5745 \\
5760 \\
5775\end{array}$ & $\begin{array}{l}12.7 \\
12.7 \\
12.7 \\
12.8 \\
12.8\end{array}$ & $\begin{array}{l}578 \\
90 \\
57 \\
90 \\
01\end{array}$ & $\begin{array}{l}5715 \\
5730 \\
5745 \\
5760 \\
5775\end{array}$ & $\begin{array}{l}128.2 \\
128.6 \\
128.9 \\
129.3 \\
129.6\end{array}$ & $\begin{array}{l}1.3 \\
1.3 \\
1.3 \\
1.3 \\
1.3\end{array}$ & $\begin{array}{l}5715 \\
5730 \\
5745 \\
5760 \\
5775\end{array}$ & $\begin{array}{l}17 \\
17 \\
17 \\
17 \\
17\end{array}$ & $\begin{array}{l}63 \\
30 \\
99 \\
69 \\
39\end{array}$ & $\begin{array}{l}5715 \\
5730 \\
5745 \\
5760 \\
5775\end{array}$ & $\begin{array}{l}281.7 \\
282.5 \\
283.2 \\
283.9 \\
284.7\end{array}$ \\
\hline $\begin{array}{l}1.434819 \\
1.440439 \\
1.446073 \\
1.451718 \\
1.457377\end{array}$ & $\begin{array}{l}5790 \\
5805 \\
5820 \\
5835 \\
5850\end{array}$ & $\begin{array}{l}12.8 \\
12.9 \\
12.9 \\
12.9 \\
13.0\end{array}$ & $\begin{array}{l}277.3292 \\
281.4179 \\
285.5662 \\
289.7756 \\
294.0470\end{array}$ & $\begin{array}{l}5790 \\
5805 \\
5820 \\
5835 \\
5850\end{array}$ & $\begin{array}{l}129.9 \\
130.3 \\
130.6 \\
130.9 \\
131.3\end{array}$ & $\begin{array}{l}1.403668 \\
1.418805 \\
1.434111 \\
1.449591 \\
1.465247\end{array}$ & $\begin{array}{l}5790 \\
5805 \\
5820 \\
5835 \\
5850\end{array}$ & $\begin{array}{l}176.7 \\
177.0 \\
177.4 \\
177.7 \\
178.1\end{array}$ & $\begin{array}{l}0.326609 \\
0.327581 \\
0.328554 \\
0.329528 \\
0.330500\end{array}$ & $\begin{array}{l}5790 \\
5805 \\
5820 \\
5835 \\
5850\end{array}$ & $\begin{array}{l}285.4 \\
286.2 \\
286.9 \\
287.6 \\
288.4\end{array}$ \\
\hline $\begin{array}{l}1.463050 \\
1.468733 \\
1.474434 \\
1.4801 .43 \\
1.485867\end{array}$ & $\begin{array}{l}5865 \\
5880 \\
5895 \\
5910 \\
5925\end{array}$ & $\begin{array}{l}13.0 \\
13.0 \\
13.1 \\
13.1 \\
13.1\end{array}$ & $\begin{array}{l}298.3809 \\
302.7788 \\
307.2409 \\
311.7686 \\
316.3631\end{array}$ & $\begin{array}{l}5865 \\
5880 \\
5895 \\
5910 \\
5925\end{array}$ & $\begin{array}{l}131.6 \\
131.9 \\
132.3 \\
132.6 \\
133.0\end{array}$ & $\begin{array}{l}1.481078 \\
1.497092 \\
1.513282 \\
1.529659 \\
1.546222\end{array}$ & $\begin{array}{l}5865 \\
5880 \\
5895 \\
5910 \\
5925\end{array}$ & $\begin{array}{l}178.4 \\
178.8 \\
179.1 \\
179.5 \\
179.9\end{array}$ & $\begin{array}{l}0.33 \\
0.33 \\
0.33 \\
0.33 \\
0.33\end{array}$ & $\begin{array}{l}5865 \\
5880 \\
5895 \\
5910 \\
5925\end{array}$ & $\begin{array}{l}289.1 \\
289.9 \\
290.6 \\
291.3 \\
292.1\end{array}$ \\
\hline $\begin{array}{l}1.491604 \\
1.497355 \\
1.503120 \\
1.508897 \\
1.514686\end{array}$ & $\begin{array}{l}5940 \\
5955 \\
5970 \\
5985 \\
6000\end{array}$ & $\begin{array}{l}13.2 \\
13.2 \\
13.2 \\
13.3 \\
13.3\end{array}$ & $\begin{array}{l}321.0249 \\
325.7554 \\
330.5550 \\
335.4252 \\
340.3672\end{array}$ & $\begin{array}{l}5940 \\
5955 \\
5970 \\
5985 \\
6000\end{array}$ & $\begin{array}{l}133.3 \\
133.6 \\
134.0 \\
134.3 \\
134.6\end{array}$ & $\begin{array}{l}1.562972 \\
1.579912 \\
1.597041 \\
1.614366 \\
1.631891\end{array}$ & $\begin{array}{l}5940 \\
5955 \\
5970 \\
5985 \\
6000\end{array}$ & $\begin{array}{l}180.2 \\
180.6 \\
180.9 \\
181.3 \\
181.6\end{array}$ & $\begin{array}{l}0.336359 \\
0.337338 \\
0.338317 \\
0.339298 \\
0.340279\end{array}$ & $\begin{array}{l}5940 \\
5955 \\
5970 \\
5985 \\
6000\end{array}$ & $\begin{array}{l}292.8 \\
293.6 \\
294.3 \\
295.0 \\
295.8\end{array}$ \\
\hline
\end{tabular}

